\title{
ACOUSTOFLUIDICS FOR DIAGNOSTICS AND INTRACELLULAR DELIVERY
}

\author{
by \\ Alinaghi Salari \\ Master of Science in Electrical Engineering, University of Calgary, Canada, 2015 \\ Master of Science in Mechanical Engineering, Sharif University of Technology, Iran, 2011 \\ Bachelor of Science in Mechanical Engineering, Ferdowsi University of Mashhad, Iran, 2008
}

A dissertation presented

to

Ryerson University

\author{
in partial fulfillment of the \\ requirements for the degree of \\ Doctor of Philosophy \\ in the program of \\ Biomedical Engineering
}

Toronto, Ontario, Canada, 2020

(C) Alinaghi Salari, 2020 


\section{AUTHOR'S DECLARATION FOR ELECTRONIC SUBMISSION OF A DISSERTATION}

I hereby declare that I am the sole author of this dissertation. This is a true copy of the dissertation, including any required final revisions, as accepted by my examiners.

I authorize Ryerson University to lend this dissertation to other institutions or individuals for the purpose of scholarly research.

I further authorize Ryerson University to reproduce this dissertation by photocopying or by other means, in total or in part, at the request of other institutions or individuals for the purpose of scholarly research.

I understand that my dissertation may be made electronically available to the public. 
Acoustofluidics for Diagnostics and Intracellular Delivery

\author{
Alinaghi Salari \\ Doctor of Philosophy \\ Biomedical Engineering \\ Ryerson University, Toronto, Canada, 2020
}

In biomedical research, there is a high demand for tools that provide high precision, costeffective, and portable methodologies for diagnostic and drug delivery purposes. The main focus of this thesis is on ultrasound techniques, where sound waves are employed for conducting in vivo and in vitro tests for different diagnostic and therapeutic applications. First, bubble-mediated ultrasound approaches for imaging are explored, and then, a bubble-free acoustofluidic strategy is proposed for in vitro intracellular delivery applications.

As a significant component of many ultrasound techniques, microbubbles have been used as contrast agents and for targeted imaging and drug delivery applications. Size, monodispersity, and stability of microbubbles are important characteristics for the effectiveness of these techniques, and therefore, various methods have been developed for producing microbubbles. In the first microfluidic approach, an expansion-mediated breakup regime is proposed that enables a controlled breakup of large bubbles into smaller size microbubbles in a microfluidic device. Also, various population distributions are reported, and the governing dimensionless numbers are identified. In the second approach, by taking advantage of the dynamics of the bubble size variation inside a gas permeable microfluidic device, the shrinkage of large bubbles into smaller size microbubbles is presented. Theoretical modeling and experimental verification are conducted to identify the design parameters governing the final size of the microbubbles. It is also shown that 
by controlling the mixing ratio of a high-molecular-weight gas with a low-molecular-weight gas, this approach could enable the production of nanobubbles.

An acoustofluidic strategy for probing cellular stiffness and facilitating intracellular delivery is also presented. Acoustic waves are employed to control the oscillations of adherent cells in a microfluidic channel. Novel observations are reported that individual cells are able to induce microstreaming flow when they are excited by controlled acoustic waves in vitro. A strong correlation between cell stiffness and cell-induced microstreaming flow is observed. Also, it is shown that the combined effect of acoustic excitation and cell-induced microstreaming can facilitate the cellular uptake of different size cargo materials. Successful delivery of $500 \mathrm{kDa}$ dextran to various cell lines with unprecedented efficiency in the range of $65-85 \%$ in a $20 \mathrm{~min}$ treatment is demonstrated. 


\section{Acknowledgements}

The author graciously thanks his $\mathrm{PhD}$ advisors, Dr. Scott Tsai from the Department of Mechanical and Industrial Engineering, and Dr. Michael Kolios from the Department of Physics, for their vision, enthusiasm, and support throughout this work.

The author would like to also thank the supervisory committee members, Dr. Stephen Waldman from the Department of Chemical Engineering at Ryerson University, and Dr. Alfred $\mathrm{Yu}$ from the Department of Electrical and Computer Engineering at University of Waterloo, for providing insightful comments throughout this work and reviewing this dissertation.

The author is deeply grateful to Dr. Sila Appak-Basky and Elizabeth Berndl who provided invaluable biology-related technical input, and to Graham Ferrier and Victoria Bulycheva for technical assistance with the equipment setup.

The author also expresses his sincere gratitude to Dr. Dan Voicu, Dr. Dario Bogojevic, Dr. Caterina Di Ciano-Oliveira, and Chris Spring for their guidance with device fabrication and imaging techniques.

Also, the author acknowledges the help and support received from Dr. Jiang Xu, Maryam Navi, Dr. Boris Hinz, Maya Ezzo, Justin Cowen, Dr. Vaskar Gnyawali, John Abousawan, Dr. Imogen Coe, Dr. Costin Antonescu, Amin Jafari Sojahrood, Hossein Haghi, Aren Gharabeiki, Dr. Raffi Karshafian, and Dr. Daniel Foster.

And special thanks to the author's colleagues, including Yan Wang, Dr. Celina Yang, Charlotte Ferworn, Dr. Morteza Jeyhani, Huma Inayat, Ali Paknahad, Intesar Zalloum, Jennifer Kieda, Katherine Chan, Liam Kerr, Daniel Wong, and Niki Abbasi. 


\section{Table of contents}

ABSTRACT ............................................................................................................................................ iii

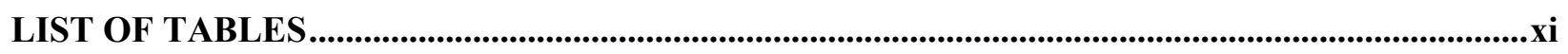

LIST OF FIGURES.............................................................................................................................................

LIST OF APPENDICES ............................................................................................................................

1 INTRODUCTION .............................................................................................................................................1

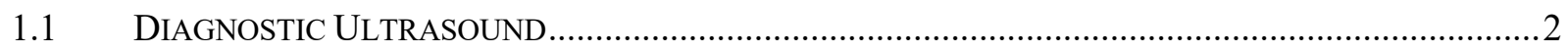

1.1.1 Bubble-Mediated Ultrasound Techniques ................................................................

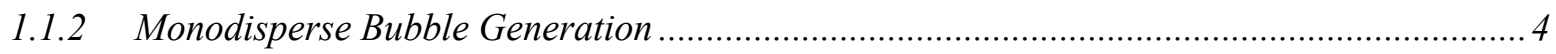

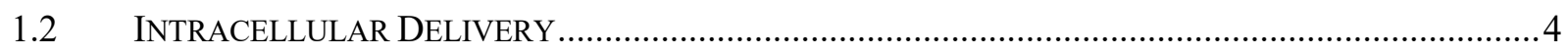

1.2.1 Mechanical Disruption Techniques.........................................................................

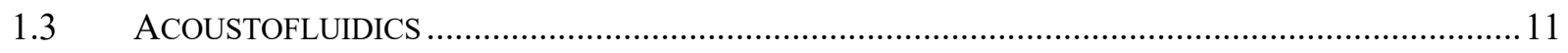

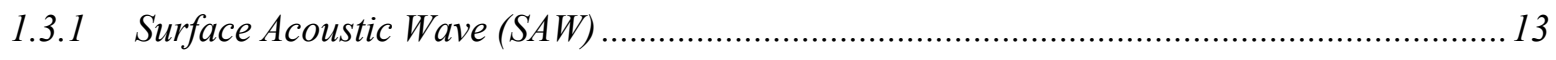

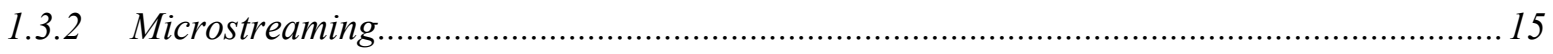

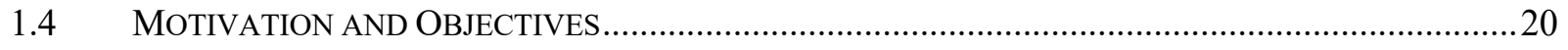

1.4.1 Microfluidic Approaches for Bubble Generation ………..............................................2

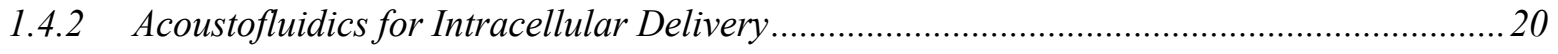

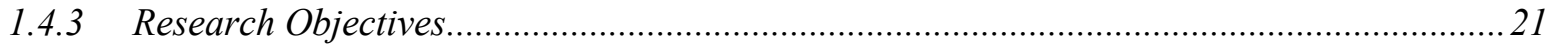


1.5 OVERVIEW OF THE DISSERTATION.

1.6 AUTHOR's CONTRIBUTION IN THE CONTEXT OF COLLABORATION .........................................26

2 BUBBLE SIZE CONTROL IN MICROFLUIDIC CHANNELS .....................................................28

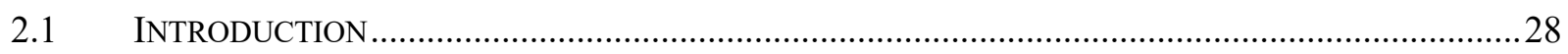

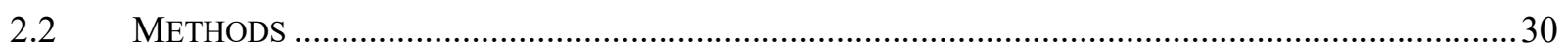

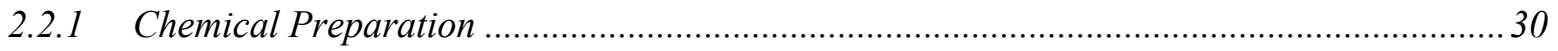

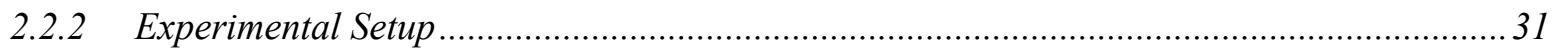

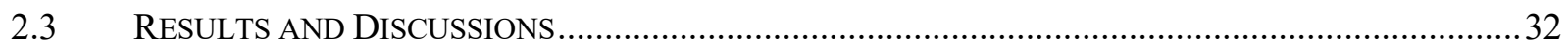

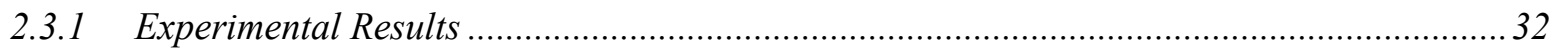

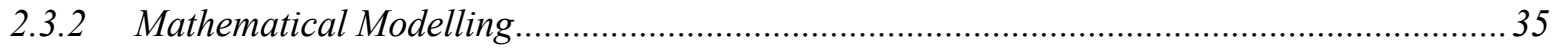

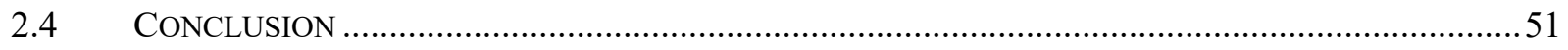

3 EXPANSION-MEDIATED BUBBLE BREAKUP IN MICROFLUIDICS.....................................53

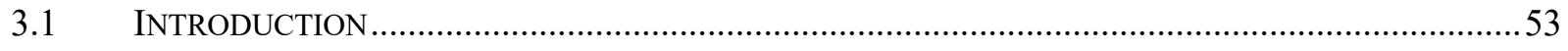

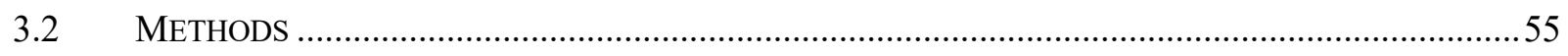

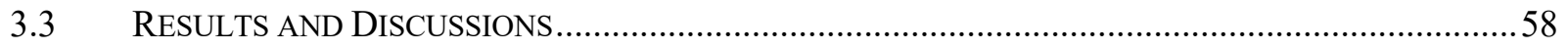

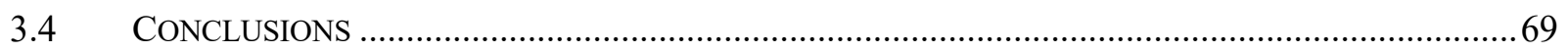

4 NANOBUBBLE GENERATION VIA CONTROLLED SHRINKAGE OF MICROBUBBLES .. 70

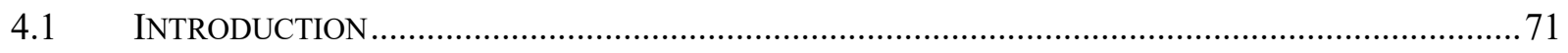

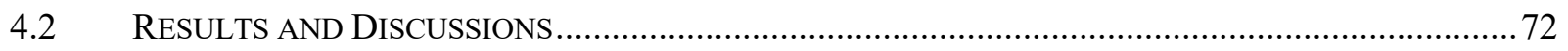

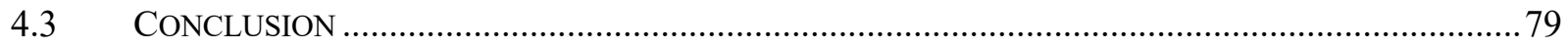


4.4 METHODS

4.4.1 Microfluidic Device Fabrication and Operation.......

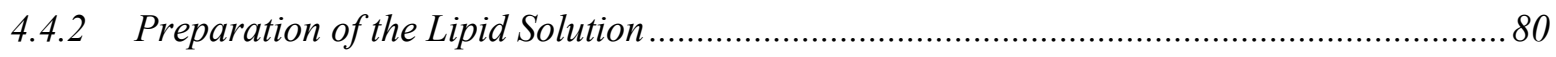

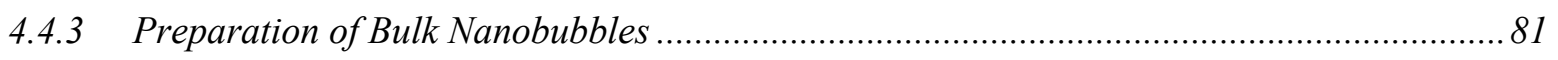

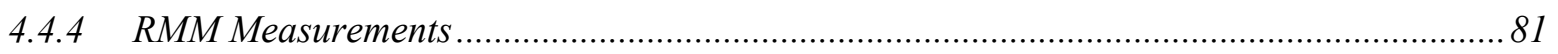

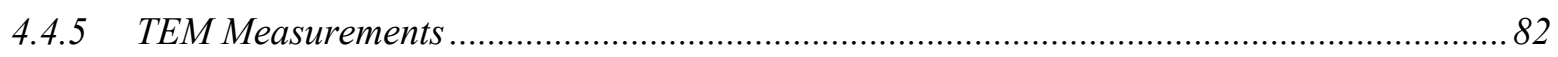

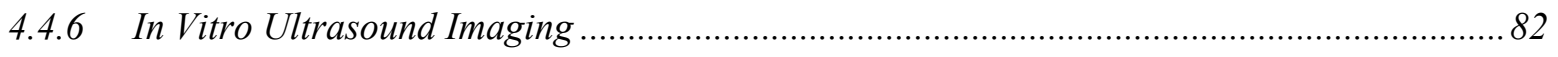

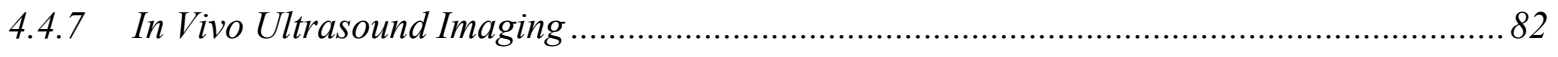

5 ACOUSTIC MICROFLOWS GENERATED BY OSCILLATING CELLS .................................83

5.1 INTRODUCTION

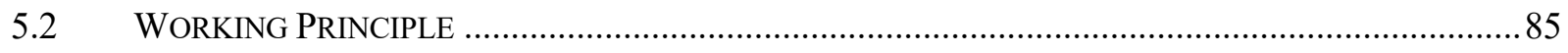

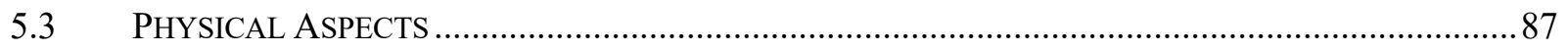

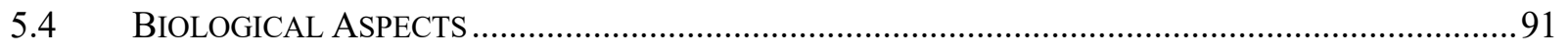

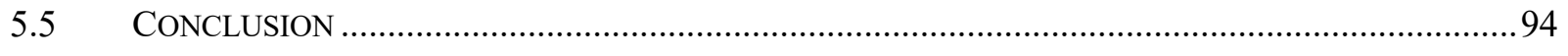

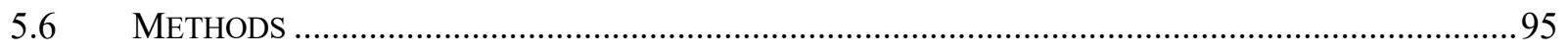

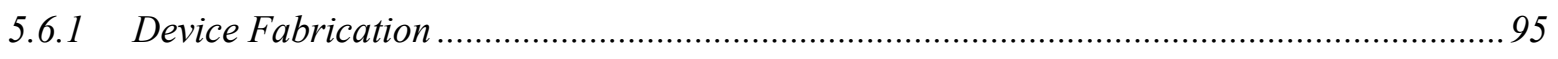

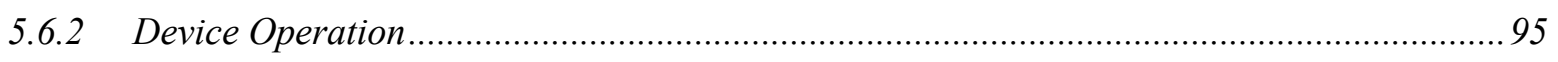

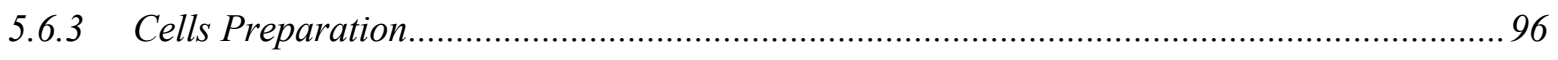

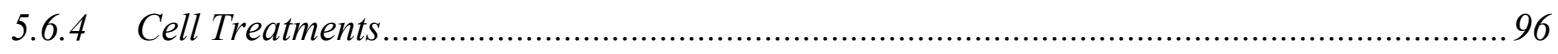




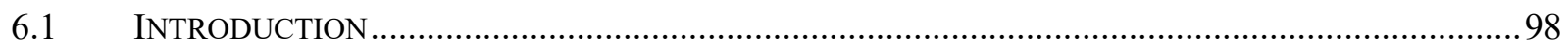

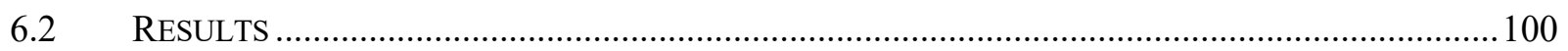

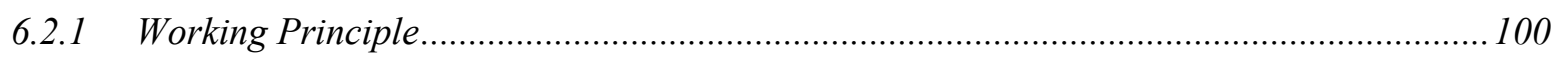

6.2.2 Effect of Different Actuation Parameters ................................................................... 102

6.2.3 Versatility in the Delivery of Various Materials into Different Cells................................ 105

6.2.4 Probing the Mechanism of Intracellular Delivery.......................................................... 107

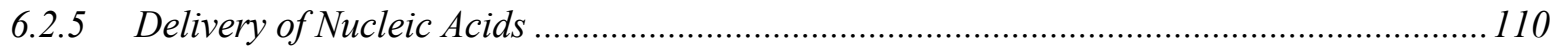

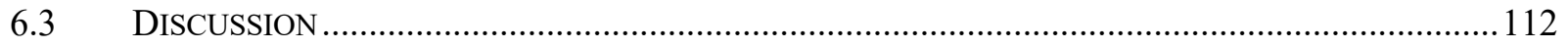

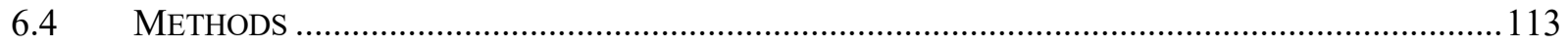

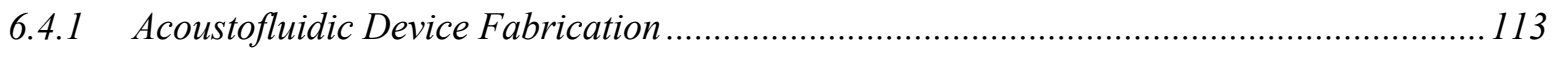

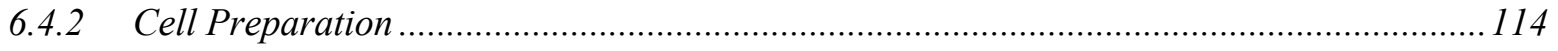

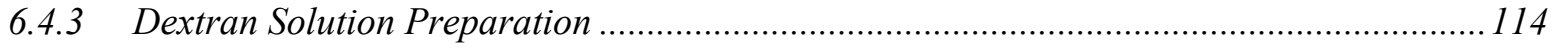

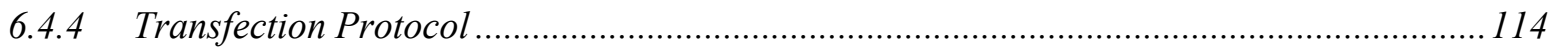

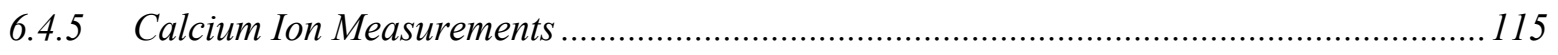

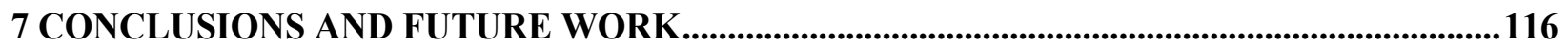

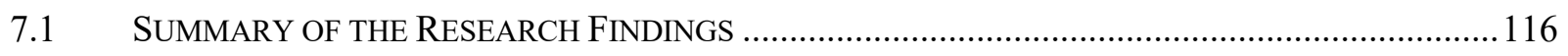

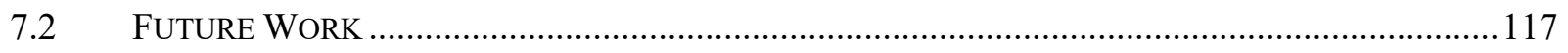




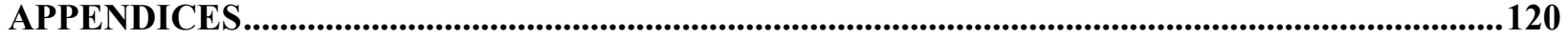

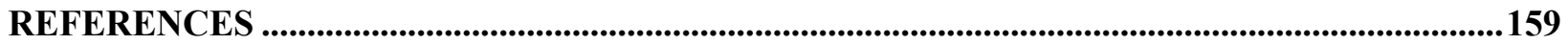




\section{List of Tables}

Table 3.1 List of the aqueous solutions and their properties used in our experiments.............................58 


\section{List of Figures}

Figure 1.1 A variety of bubble-mediated effects used in ultrasound-based imaging and drug delivery techniques. (a) Detectable backscattered signals at moderate acoustic pressures. (b) The microstreaming effect due to stable cavitation of the bubble. (c) Fragmentation of a bubble into smaller daughter bubbles as a result of high acoustic pressures. (d) Radiation force directing the bubble away from the acoustic source or attracting it towards the other bubbles. (e) Inertial cavitation occurring as a result of high acoustic pressures. (f) Dissolution caused by acoustic pressures at the levels below the fragmentation threshold. Adapted from Sirsi and Borden. ${ }^{9}$

Figure 1.2 A schematic of different membrane-disruption-based strategies for intracellular delivery using (a) solid mechanical contact, (b) electric field, (c) shear stress, and (d) photo-thermal effect. Adapted from Stewart et al. ${ }^{18}$

Figure 1.3 Mechanical penetration techniques for intracellular delivery. (a) The working principle of a microfluidic single-cell microinjection system where a cell is moved towards a fixed microneedle, while valve V1 is open and V2 is closed. Once the penetration occurred, the cell is moved to the outlet through valve V2 when V1 is closed. (b) A nanostraw-based device coupled with electroporation mechanism. The cargo molecules move into the cell cytoplasm through the nanostraws while the localized electric field facilitates this process. (c) SEM images of vertical silicon nanowires fabricated by (i) chemical vapor deposition (CVD) and (ii) reactive ion etching. The scale bars represent $1 \mu \mathrm{m}$. Schematic of (iii) early and (iv) late stages of cell penetration using the nanowires. Adapted from Shalek et al., ${ }^{38}$ Xie et al., ${ }^{39}$ Adamo and Jensen. ${ }^{40}$

Figure 1.4 Examples of shear based permeabilization of the cell membrane. (a) A schematic of different aspects of sonoporation. Pulling, pushing, and microstreaming (i.e., shear) are caused by stable cavitation, whereas jetting is induced by inertial cavitation. The translational motion is due to the acoustic radiation force. Sonophoresis is induced mainly by shock waves as a result of low-frequency ultrasound. (c) A shear-mediated intracellular delivery based on the rapid deformation of cells when they pass through a microfluidic constriction. Adapted from Sharei et al. ${ }^{43}$ and Kotopoulis et al. ${ }^{57} .10$

Figure 1.5 Acoustic streaming flow generated by the radiated sound waves at the Rayleigh angle into the fluid. Adapted from Frommelt et al. ${ }^{75}$ C 2008 IEEE. 
Figure 1.6 Schematics of microstreaming flows generated next to an oscillatory bubble with an initial radius $R 0$. A steady streaming flow is generated outside the boundary layer due to the recirculating flows within the boundary layer. The amplitude of translational and volume oscillations is equal to $\varepsilon R 0$. The dashed red line represents the boundary layer, which has a thickness of $\delta . \mathrm{r}$ and $\theta$ are the polar coordinates.

Figure 2.1 A schematic design of the microfluidic system containing a serpentine liquid-filled channel with a total length of $350 \mathrm{~mm}$. Microbubbles are generated at a $20 \mu \mathrm{m}$ width junction, where flows of air (central channel, grey arrow) and aqueous solution (side channels, red arrows) meet orthogonally. Vacuum is applied through two connections to achieve a uniform vacuum pressure across the entire chip. The bubble size variation is monitored by moving the microscope within the area depicted by red dashed lines. Images are captured when bubbles pass through this area.

Figure 2.2 A plot of the experimental results for the bubble volume $V$ versus the location $l$, along the microchannel at ten different vacuum pressures. The error bars indicate the standard deviations calculated by analysing $>10$ bubbles for each data point. In all experiments, the mixture surface tension, aqueous liquid flow rate, and air pressure are kept constant at $\sigma=36.60 \mathrm{mN} \mathrm{m}-1$, $4 \mu \mathrm{L} \min -1$, and $27.6 \mathrm{kPa}$, respectively. The plot shows a monotonic trend of increasing microbubble shrinkage rate with increasing vacuum pressure.

Figure 2.3 Experimental results for the bubble volume $V$ versus the location along the microchannel $l$ for aqueous solutions with three different surface tensions. The error bars show standard deviations calculated by analysing $>10$ bubbles for each data point. Here, the vacuum pressure is held constant at (a) $P v=-50 \mathrm{kPa}$, and (b) $P v=-87 \mathrm{kPa}$. In all experiments, the aqueous liquid flow rate and air pressure are kept constant at $4 \mu 1 \mathrm{~min}^{-1}$ and $27.6 \mathrm{kPa}$, respectively. Bubbles generated at higher surface tensions are initially larger and shrink more throughout the channel. .35

Figure 2.4 Microscopy images showing the fluid channel, PDMS bulk, and the vacuum channel. (a) The schematic profile overlaid on the images, of absolute pressure distribution and (b) the concentration distribution across the microfluidic channel. The changes of absolute pressure and air concentration through the PDMS bulk is negligible. Ostwald's law governs the relationship between the absolute pressure and air concentration in the fluid channel, and pressure and air concentration in the bulk PDMS. (c) A schematic diagram of the concentration changes across a microbubble shell. .39 
Figure 2.5 Simulation results representing the concentration of dissolved air along the channel centreline at three different vacuum pressures, $P v=0,-50$ and $-87 \mathrm{kPa}$. In simulations, we assume that the microfluidic channel is filled with water only, and thus the effect of bubble flow is neglected. The effect of channel entrance on the air concentration within the liquid is diminished once the liquid travels $50 \mathrm{~mm}$ along the channel length. The inset shows the 2D geometry used in the simulation and the boundary conditions applied. 44

Figure 2.6 Dimensionless experimental and modeling results for the bubble volume VVinitial versus the location along the channel lltotal. The results are shown for different surface tensions $\sigma$, while vacuum pressures (a) $P v=-50 \mathrm{kPa}$, and (b) $P v=-87 \mathrm{kPa}$. (c) Three different vacuum pressures with constant surface tension $\sigma=36.60 \mathrm{~m} \mathrm{Nm}-1$. Data points state experimental results, while the solid lines represent the numerical solution of the mathematical model. The modeling results demonstrate a very good agreement with the experimental data. Here, initial bubble volume Vinitial $=43 \pi R 03$ and the microchannel length ltotal $=350 \mathrm{~mm}$. In (a) and (b), initial bubble radius $R 0=89 \mu \mathrm{m}, 83 \mu \mathrm{m}$, and $61 \mu \mathrm{m}$, correspond to surface tensions $\sigma=48.29 \mathrm{~m} \mathrm{Nm}-$ $1,43.41 \mathrm{~m} \mathrm{Nm}-1$, and $36.60 \mathrm{~m} \mathrm{Nm}-1$, respectively. In (c), the initial bubble radius $R 0=63 \mu \mathrm{m}$.

Figure 2.7 A plot of the dimensionless bubble volume VVinitial versus the position along the channel lltotal. Here, the dimensionless parameters are $\sigma=1.18 \times 10-2, P v=-0.5, E=2.90 \times 10-3$, $k g=1.27$, and $f=0.62$. The approximated solution (Eq. (2.20)) is almost identical to the exact solution (Eq. (2.18)). .49

Figure 2.8 The dependence of shrinkage time required for bubbles to reach a radius $R=0.01$ (upper curve) and $R=0.1$ (lower curve) given an initial radius, $R 0$, on vacuum pressure. The vacuum pressure $P v$ is more effective on initially smaller bubbles. .50

Figure 3.1 (a-b) Schematic diagram of the device showing a microfluidic flow-focusing geometry coupled with an expansion region at the breakup junction. First, air microbubbles (shown in black color) are generated at the flow-focusing cross-junction and then travel through the connecting channel, which leads to the expansion region. The two geometries used in this study have identical dimensions except at the breakup junction. In (a), the connecting channel has a uniform width $w=40 \mu \mathrm{m}$. In (b), the connecting channel width tapers from $40 \mu \mathrm{m}$ to a width $w=20 \mu \mathrm{m}$ at the breakup junction, over a $100 \mu m$ distance that begins $300 \mu m$ upstream from the breakup junction. At specific values of certain 
geometrical and flow parameters, the microbubbles are squeezed at the entrance of the expansion region, and thus, periodically break into smaller daughter bubbles (shown in blue). $w$ and $W$ represent the widths of the breakup junction and expansion region, respectively. The continuous and dispersed phases are shown in gray and black colors, respectively. (c-e) Different flow regimes observed in our bubble generation experiment. Here, the geometry has the breakup junction width $w=40 \mu \mathrm{m}$ and the experiment uses two different aqueous solutions: (c) Aq-b1; (d) Aq-b2 (see Table 3.1). At an aqueous flow rate $Q w>5 m L h r-1$, increasing the gas pressure $P g$ eventually leads to the transition from mono-disperse bubbles to periodic symmetric or asymmetric breakup, and then random breakup. (e) Microscope images of each flow regime in this experiment. The scale bars represent $50 \mu \mathrm{m}$. .56

Figure 3.2 (a-c) Images of different modes of periodic breakup occurring at the entrance of the expansion region. Intact bubbles are shown in black, whereas daughter bubbles that result from breakup, are in blue. (a) The periodic symmetric mode, and $(b, c)$ the periodic asymmetric mode. The asymmetric mode can be further categorized into two sub-modes A and B, which occur consecutively one after another, meaning that every asymmetric A sub-mode is followed by an asymmetric B sub-mode. As shown, the presence of the pre-arrived bubbles " 1 " and " 2 " is crucial for the breakup of the " 3 "rd bubble. Each bubble is elongated to its maximum length of $\mathrm{lm}$, once it enters the expansion region. In a sequence of three arriving bubbles, only one bubble is elongated to its critical length of $l c r$, at which point breakup occurs. The images in (a) are from experiments that use Aq-b1, and in (b, c) Aqb2 is used. (d) A plot of the volume ratio of the daughter bubbles (top graph), and dimensionless critical length immediately before breakup (bottom graph) versus the inverse Ohnesorge number Oh 1. The value of $O h-1$ determines the periodic breakup mode. As shown, for each geometry, a decrease in $\operatorname{lcr} D$ is observed by increasing $O h-1$, suggesting that the effect of shear force becomes significant in asymmetric breakup. However, in the symmetric mode, shear appears to be a secondary dominant force. All data depicted in (d) are collected at the transition pressure of $P g, t$, the point at which the mono-disperse regime changes to the periodic breakup regime (see Figure 3.1c and d)...59

Figure 3.3 Different output populations obtained in our bubble generation experiments. Here, the geometry with the breakup junction width $w=40 \mu \mathrm{m}$ and continuous phase Aq-b1 is used. (a) $P g=100 \mathrm{kPa}$, $Q w=5 \mathrm{~mL} \mathrm{hr}-1$; $\quad$ (b) $P g=160 \mathrm{kPa}, \quad Q w=10 \mathrm{ml} \mathrm{hr}-1$; $\quad$ (c) $P g=180 \mathrm{kPa}, \quad Q w=$ $10 \mathrm{~mL} \mathrm{hr}-1$. As shown, using a single geometry, we obtain (a) mono-disperse, (b) bi-disperse, or (c) tri-disperse populations only by controlling the aqueous phase flow rate and gas pressure. In (b) and (c), one in every three bubbles breaks. Therefore, the number of intact bubbles in (b) is 
approximately equal to the number of daughter bubbles, and in (c) the number of intact bubbles is approximately two times greater than the number of daughter bubbles .65

Figure 3.4 (a-c) Different flow regimes observed in our dodecane droplet generation experiment for the geometry of breakup junction width $w=40 \mu \mathrm{m}$ and two different aqueous solutions: (a) Aq-d1; (b) Aq-d2 (see Table 3.1). To be consistent with the results presented in Figure 3.1c and d, here, we report the dispersed phase pressure versus continuous phase flow rate. At an aqueous flow rate $Q w>$ $0.1 m L h r-1$, increasing the oil pressure $P g$ leads to the transition from mono-disperse bubbles to periodic symmetric or asymmetric breakup, and then random breakup. (c) Microscope images of each flow regime in this experiment. Droplet breakup regimes exhibit similar trends of mono-disperse, periodic symmetric, and asymmetric breakup that are observed in bubble breakup experiments (see Figure 3.1c and d). (d) The frequency at which dispersions arrive at the expansion region, $f$, versus the product of Weber number and the squared Capillary number, WeCa2. Here, the dashed curve $\kappa W e 0.1 \mathrm{Ca} 0.2$ is fitted to the experimental data points obtained from bubble experiments. The inset shows a log-log plot of $f$ versus WeCa2, where the dashed line shows the scaling, $f 0.5=$ $\kappa W e 0.1 \mathrm{Ca} 0.2$. All data depicted here are collected at the transition pressure of $\mathrm{Pg}, t$, at which point the mono-disperse regime changes to periodic breakup regime (see Figure 3.1c and $\mathrm{d}$ and Figure 3.4a and $\mathrm{b}$ ). The proportionality constant $\kappa$ for bubble, dodecane oil droplet, and mineral oil droplet experiments is $1.2 \times 102,2.9 \times 102$, and $6.0 \times 101$, respectively. The bubble and droplet experimental results show a good agreement with the scaling, $f 0.5 \propto W e 0.1 \mathrm{Ca0} .2$. The scale bar represents $50 \mu \mathrm{m}$.

Figure 4.1 (a) Schematic diagram of the microfluidic nanobubble generator featuring a microbubble generation platform with a serpentine shrinkage channel. There are 50 turns in the serpentine channel, and the total serpentine channel length is $620 \mathrm{~mm}$; Dimensions of the cross-junction are: orifice width $\mathrm{W}=20 \mu \mathrm{m}$, channel height $\mathrm{h}=50 \mu \mathrm{m}$, and serpentine channel width $\mathrm{W}=350 \mu \mathrm{m}$; Dissolution of $\mathrm{N}_{2}$ from the mixed gas core microbubbles leads to the shrinkage of the microbubbles down to the nanoscale. Insoluble $\mathrm{C}_{3} \mathrm{~F}_{8}$ constitutes the final equilibrium gas core of nanobubbles. (b) Bright-field images of the shrinkage of $4.2 \mathrm{wt} \% \mathrm{C}_{3} \mathrm{~F}_{8}$ in air microbubbles within the serpentine channel. The channel numbers, \#10, \#20,\#30, \#40, and \#50, represent the number of times that the serpentine channel has turned. Each turn has a distance of $12 \mathrm{~mm}$ and thus the channel number indicates the distance from the bubble generation orifice. A longer distance corresponds to a longer travelling time. (c) A plot of the ratio of the normalized final bubble volume $V_{f} / V_{i}$, versus the dissolution time of the bubbles. Here, $V_{f}$ and $V_{i}$ are the final and initial bubble volume, respectively. All of the initial bubble 
volumes of different gas compositions are kept approximately constant. As shown, a decrease in $V_{f} / V_{i}$ is observed by decreasing the mass percent of $\mathrm{C}_{3} \mathrm{~F}_{8}$ in the gas mixture. Each data point shows the average of 3 measurements, and the error bars represent one standard deviation. 73

Figure 4.2 Shrinkage of microbubbles of different initial sizes, with the gas composition kept constant. (a) Bright-field images of different initial size microbubbles along the serpentine channel. The leftmost images are taken at the orifice, and the labels \#5,\#10,\#15, and \#20 correspond to the serpentine turn number. a1, a2, and a3 are the initial bubble diameters, $D_{i}=28.3 \mu \mathrm{m}, 24.6 \mu \mathrm{m}$, and $21.5 \mu \mathrm{m}$, respectively. (b) A plot of bubble diameter $D$ versus evolution time. (c) A plot of bubble final diameter $D_{f}$ versus initial diameter $D_{i}$. (d) Size measurements of final nanobubbles of a1, a2, and a3 by RMM and morphology characterizations by TEM. In TEM images, the white arrows point to individual bubbles as examples of monodisperse bubbles in MF-NBs and polydisperse bubbles in Bulk-NBs. Each size measurement was repeated three times. In these experiments an industrial gas mixture of $0.17 \mathrm{wt} \% \mathrm{C}_{3} \mathrm{~F}_{8}$ in $\mathrm{N}_{2}$ is used. Bulk nanobubbles are taken from the $1 \mathrm{~mL}$ bottom layer of a $3 \mathrm{~mL}$ initial bulk nanobubbles solution, made by high frequency mechanical agitation, after centrifugation at 500 rpm for 2 minutes, based on a modified version of a previously reported protocol. ${ }^{194,195}$ .75

Figure 4.3 In vitro and in vivo contrast-enhanced ultrasound imaging with nanobubbles. Ultrasound signals are generated and collected by an $18 \mathrm{MHz}$ MS250 transducer (a) In vitro ultrasound images of nanobubbles under different contrast modes: B-mode (left), and non-linear mode (right), in a bloodvessel-like phantom. The nanobubbles are characterized by RMM: The MF-NBs have a diameter of $328 \mathrm{~nm} \pm 49 \mathrm{~nm}(\mathrm{n}=3)$, a concentration of $8.07 \times 10^{7} \mathrm{~mL}^{-1}$, and a polydispersity index of 0.148 ; The Bulk-NBs-1 have a diameter of $418 \mathrm{~nm} \pm 121 \mathrm{~nm}(\mathrm{n}=3)$, a concentration of $7.67 \times 10^{7} \mathrm{~mL}^{-1}$, and a poly-dispersity index of 0.288. (b) In vivo images of nanobubbles in the kidney of a mouse captured in the brightness mode (i.e., B-mode) and non-linear ultrasound mode. The white arrow points to the shadowing effect due to ultrasound attenuation by Bulk-NBs-2. The images are taken at $0 \mathrm{~s}$ and $10 \mathrm{~s}$ after the injection of the nanobubble solution. Bulk-NBs-1 are diluted 1,000 times from Bulk-NBs-2 to match the concentration of MF-NBs. MF-NBs are generated using the $0.16 \mathrm{wt}_{0} \mathrm{C}_{3} \mathrm{~F}_{8}$ in $\mathrm{N}_{2}$. BulkNBs- 1 and Bulk-NBs-2 are generated using pure $\mathrm{C}_{3} \mathrm{~F}_{8}$ gas. Mouse kidneys in all experiments are directly exposed to the ultrasound transducer. The ultrasound imaging for contrast mode is at $4 \%$ power $(2 \mathrm{MPa})$, and for $\mathrm{B}$-mode is at $1 \%$ power $(0.9 \mathrm{MPa})$.

Figure 5.1 (a) Schematic diagram (not to scale) of cell oscillation driven by acoustic waves. A thin-walled PDMS channel contains the cells. The acoustic waves are generated by a PZT disk attached onto the 
substrate next to the channel. Acoustic streaming flow is generated as a result of cell membrane oscillations. (b) Confocal images of fluorescent particle tracers taken at different heights from the substrate surface in a channel with an overall height of $25 \mu \mathrm{m}$. The MCF-7 cell is shown in the brightfield view and is outlined by the red dashed line. As depicted, a circular flow is generated in the liquid bulk above the cell, which has its maximum velocity near the channel mid height at $z \approx 14-21 \mu \mathrm{m}$. The streaming velocity approaches zero near the top and bottom surfaces of the channel due to no-slip boundary conditions. (c) The streaming flow is also simulated numerically for two model cells: a round and a flat cell. As illustrated, helical-shape streamlines are induced surrounding each cell. The round cell's helical streamlines have a higher pitch than the streamlines of the flat cell. Regions of maximum streaming flow are located close to the cell membrane. The color legend shows the nondimensionalized velocity magnitude, and the arrows depict the 2D velocity field on a cross-section of the flow. The scale bars represent $10 \mu \mathrm{m}$. The schematic in (a) is created with Biorender.com. .86

Figure $5.2(\mathrm{a}-\mathrm{c})$ Different streaming flow regimes generated surrounding two MDA-MB-231 cells at different actuation frequencies. $1 \mu \mathrm{m}$ polystyrene beads are used as flow tracers. (a) Two almost symmetric sets of out-of-plane vortices are generated on two sides of each cell when excited at $\sim 32$ $\mathrm{kHz}$, as opposed to (b) planar vortices which form over the entire cell surface at $\sim 73 \mathrm{kHz}$. (c) The flow regime changes to a combination of in- and out-of-plane motion at higher frequencies (e.g., $\sim 98$ $\mathrm{kHz}$ ). (d) As the bead diameter increases, the acoustic radiation force becomes the dominant force acting on the bead, causing (d-left panel) a $5 \mu \mathrm{m}$ and (d-right panel) a $10 \mu \mathrm{m}$ polystyrene bead to partially follow the flow streamlines and eventually remain stationary on the boundary between the cell and substrate. The blue arrows in (d) show the final location of the beads. White dashed lines show the cells' outlines. The scale bars represent $10 \mu \mathrm{m}$.

Figure 5.3 The maximum streaming velocity induced by MDA-MB-231 cells under different conditions. (a) Substrates with different thicknesses in the range of $100 \mu \mathrm{m}$ to $1 \mathrm{~mm}$. Increasing the substrate thickness decreases the streaming velocity. (b) Different actuation voltages in the range of 1 to $6 \mathrm{~V}$ (rms). A higher actuation voltage corresponds to a higher oscillation amplitude of the substrate, which is coupled to the cell, causing larger amplitude cell oscillations and a stronger microstreaming effect. (c) Trypsinization over $4 \mathrm{~min}$. As the adhesion proteins are dissociated and the projected area of the cells decreases, the cell attachment to the substrate is weakened, causing the streaming velocity to decrease. The streaming flow, however, is recovered within $24 \mathrm{hr}$ post-treatment. (d) Osmotic pressure variations over $10 \mathrm{~min}$. Cell exposure to hypotonic solution results in an increase in the cell volume and changes in cell physical properties, which in turn results in a decrease in the streaming velocity. 
The streaming flow can be recovered within $1 \mathrm{hr}$ post-treatment. (e) Treatment with $4 \mu \mathrm{M}$ Cytochalasin D. When F-actin proteins are disrupted, the cell loses its stiffness, and consequently, the streaming velocity decreases. This change in streaming flow is recovered within $5 \mathrm{hr}$ post-treatment. (f) Fixation with 4\% Paraformaldehyde. The cross-linked structure formed upon fixation causes the entire cell to oscillate as a uniform meshwork which yields a stronger streaming flow. The error bars represent one standard deviation $(\mathrm{n} \geq 10, \mathrm{p} \leq 0.05)$. .90

Figure 6.1 Schematic of the acoustofluidic device showing the hypothetical mechanism of intracellular delivery. Due to the actuation of the PZT transducer, acoustic waves are generated and propagated through the substrate. The excitation of cells within an ROI induces the formation of acoustic microstreaming, which in turn facilitates the transport of cargo material from the bulk of the liquid to the cell membrane. As a result of the combined effect of acoustic excitation and microstreaming flow, the cargo material is taken up by the cell. The schematic is created with Biorender.com.

Figure 6.2 The effect of input power and treatment duration on the intracellular delivery. (a) The delivery results for different treatment duration when continuous $1 \mathrm{~W}$ input power is applied. (b) Delivery results for the input power in the range of $0.1-1 \mathrm{~W}$ and $20 \mathrm{~min}$ treatment. Within this range, the temperature rise is insignificant, and therefore, a continuous wave actuation is used. (c) Delivery results for different input powers in the range of 3-21 W. For each input power, the duty cycle of the square-waves is set in the range of $7-43 \%$ for 10 min treatment duration, to avoid excessive heat generated by the transducer and the vibrating PDMS. (d) Bright-field and fluorescent images showing the cellular uptake in control and treated cells. The white arrows point to individual cells as examples of those receiving cargo material as a result of disruptions to the cell membrane. In all experiments, 3 $\mathrm{kDa}$ dextran is used as the cargo material for delivery into MDA-MB-231 cells. The scale bar represents $100 \mu \mathrm{m}$. The error bars indicate the standard deviation of three independent experiments.

Figure 6.3 The versatility of intracellular delivery of dextran with various sizes into different cells. (a) Delivery results for dextran with different molecular weights into MDA-MB-231 cells. (b) Delivery results for MCF-7 and PC3 cells. Delivery efficiency of $\sim 63 \%$ is obtained in both experiments. (c) Bright-field and fluorescent images showing $500 \mathrm{kDa}$ dextran delivery into MDA-MB-231 cells. The presence of localized high-intensity aggregates of fluorescein dextran suggests an endocytic-dominant mechanism of delivery. (d) Confocal images of living MDA-MB-231 cells exhibiting the uptake of fluorescein-conjugated $500 \mathrm{kDa}$ dextran. Plasma membrane and cell nucleus are stained with wheat 
germ agglutinin (WGA) and Hoechst 33342, respectively. The scale bars represent $100 \mu \mathrm{m}$ in (c) and $20 \mu \mathrm{m}$ in (d). A 20 min continuous square-wave at $1 \mathrm{~W}$ input power is used in all experiments. The error bars indicate the standard deviation of three independent experiments. 106

Figure 6.4 Probing possible mechanisms of delivery. (a) The intracellular level of Fluo-4 AM, a labeled calcium indicator, in control and treated cells. (b) Effect of $\mathrm{Ca}^{+2}$ on the delivery of $3 \mathrm{kDa}$ and $70 \mathrm{kDa}$ dextran. (c) Delivery results of dextran with different charges and a pH-sensitive fluorescently labeled dextran (i.e., pHrodo Green $10 \mathrm{kDa}$ dextran), which fluoresces at low pH values. (d) Exposure to continuous flow (shear stress only) and acoustic excitation on the delivery efficiency for different exposure durations. Acoustically actuated cells exhibit significantly higher delivery efficiencies compared to those exposed to shear stress only. (e) Fluorescent images of the cells acoustically treated at $1 \mathrm{~W}$ and those exposed to the continuous flow. The scale bar represents $100 \mu \mathrm{m}$. Acoustic treatments are conducted on MDA-MB-231 cells using a continuous square-wave at $1 \mathrm{~W}$ input power. Results shown in $(\mathrm{a}-\mathrm{c})$ are for $20 \mathrm{~min}$ acoustic treatments. The error bars indicate the standard deviation of three independent experiments.

Figure 6.5 Intracellular delivery of nucleic acids. (a) Delivery results of siRNA and plasmid. (b) Fluorescent images of the delivered siRNA and plasmid for control and treated cells. The scale bar represents $100 \mu \mathrm{m}$ in (b). Treatments are conducted on MDA-MB-231 cells using a 20 min continuous square-wave at $1 \mathrm{~W}$ input power. The error bars indicate the standard deviation of three independent experiments 


\section{List of Appendices}

APPENDIX A: SUPPORTING INFORMATION FOR CHAPTER 2 120

Figure A.1 Comparison of bubble shrinkage results along the microfluidic channel for a degassed PDMS device, and a saturated PDMS device left in atmospheric condition for 24 hours prior to usage. In these experiments, the aqueous liquid flow rate and air pressure are $3 \mu \mathrm{Lmin}-1$ and $25.5 \pm 1.5 \mathrm{kPa}$, respectively. The vacuum pressure $\mathrm{Pv}=0$.

APPENDIX B: SUPPORTING INFORMATION FOR CHAPTER 3.........................................121

APPENDIX C: SUPPORTING INFORMATION FOR CHAPTER 4 122

Figure C.1 A schematic of the custom-made setup for mixing gasses.

APPENDIX D: SUPPORTING INFORMATION FOR CHAPTER 5 123

Figure D.1 Confocal images of the two MDA-MB-231 cells studied in Section 2. The cells having an equal height of $\sim 10 \mu \mathrm{m}$ are stained with Actin-GFP. On each cell, the dashed red line roughly outlines the region of the cell's maximum height. The center of this region (i.e., the point of maximum height) is marked by a blue arrow. The scale bars represent $20 \mu \mathrm{m}$.

Figure D.2 The results of three different trials of maximum microstreaming velocity measurements of MDA-MB-231 cells $(n=10)$.

Figure D.3 (a-b) Schematic diagram (not to scale) of the two different device configurations. (a) PZT transducer and the microfluidic channel are attached onto the same surface of the substrate. (b) PZT transducer and the microfluidic channel are attached onto the opposite surfaces of the substrate. Theoretically, cells on either surface of the substrate can be excited by Lamb waves, regardless of the surface on which the acoustic waves are introduced. (c) The results of streaming flow induced by the same cells (i.e., MDA-MB-231) in these two device configurations. An acoustic field can form in the substrate regardless of the surface on which the transducer is 
attached, and thus, cells can exhibit microstreaming flow in either of the two device configurations. The actuation frequency is $98 \mathrm{kHz}$.

Figure D.4 Projected area measured by phase-contrast microscopy during the treatment of MDA-MB231 cells with trypsin. The error bars represent one standard deviation $(\mathrm{N} \geq 10)$. 125

Figure D.5 Cell volume measurements before and after an osmotic shock is imposed on the MDAMB-231 cells. The cells are first stained with Actin-GFP, and then fluorescent images at equal $z$ steps are taken. The volume of each cell is estimated by adding all cross-sectional area obtained from the fluorescent images. The error bars represent one standard deviation $(\mathrm{N} \geq 7)$. 125

Figure D.6 MDA-MB-231 cells stained with Phalloidin showing actin filament distribution before and after treatment with $4 \mu \mathrm{M}$ Cytochalasin D. As shown, actin filaments are depolymerized after the treatment and coagulated randomly in localized regions, as opposed to the untreated cells, where, the cells exhibit a more uniform distribution of actin filaments throughout their structure. The scale bar represents $20 \mu \mathrm{m}$. 126

Figure D.7 Young's elastic modulus measurements using AFM. Live (control) cells exhibit an average Young's modulus of $1.38 \mathrm{kPa}$. Treatment with Cytochalasin D results in a $\sim$ three-fold lower average Young's modulus of $0.48 \mathrm{kPa}$, whereas those chemically fixed with PFA are stiffer than controls with a $\sim$ five-fold higher average Young's modulus of $6.57 \mathrm{kPa}$. The error bars represent one standard deviation of all processed force measurements $(\sim 500$ per cell $)$ of four cells per group.

Figure D.8 The maximum streaming velocity induced by individual MDA-MB-231 cells monitored before and after treatment with PFA solution. The error bars represent one standard deviation.

Table D.1 The property values used in the numerical simulation 130

Figure D.9 (a) Bright-field image of an MDA-MB-231 cell. (b) Stacked image showing the tracks of $1 \mu \mathrm{m}$ beads in the liquid once the cell is driven at $98 \mathrm{kHz}$. (c) The output of the semi-automated single-particle tracking technique showing the bead tracks. The flow patterns detected by the 
automated technique match those found by the stacked image. The color bar represents the mean velocity (in pixel per frame). The scale bar represents $20 \mu \mathrm{m}$.

Figure E.1 Sample (a) bright-field and (b) fluorescent images taken from the regions of interest. (c) The outline of the cells on the bright-field image is selected using ImageJ. (d) The selected area is then transferred to the fluorescent image for further intensity calculations. The scale bar represents $100 \mu \mathrm{m}$ 136

Figure E.2 The effect of input power and treatment duration on intracellular delivery. (a) The delivery results for different treatment duration when continuous $1 \mathrm{~W}$ input power is applied. (b) Delivery results for the input power in the range of $0.1-1 \mathrm{~W}$ and 20 min treatment. (c) Delivery results for different input powers in the range of 3-21 W. For each input power, the duty cycle of the squarewaves is set in the range of $7-43 \%$ for $10 \mathrm{~min}$ treatment duration, to avoid excessive heat generated by the transducer and vibrating PDMS. In all experiments, $3 \mathrm{kDa}$ dextran is used as a cargo material for delivery into MDA-MB-231 cells. The mean intensities are normalized by the values of control experiments. The error bars indicate standard deviation of three independent experiments. 137

Figure E.3 The versatility of intracellular delivery of dextran with various sizes and charges into different cells. Delivery results for dextran (a) with different molecular weights and (b) charges into MDA-MB-231 cells. Delivery results for (c) MCF-7 and (d) PC3 cells. A 20 min continuous square-wave at $1 \mathrm{~W}$ input power is used in all experiments. The mean intensities in (b-d) are normalized by the values of control experiments. The error bars indicate standard deviation of three independent experiments. 138

Figure E.4 Probing possible mechanisms of delivery. (a) Effect of $\mathrm{Ca}^{+2}$ on the delivery of $3 \mathrm{kDa}$ and $70 \mathrm{kDa}$ dextran. (b) Delivery results of pHrodo Green $10 \mathrm{kDa}$ dextran, which fluoresces at low $\mathrm{pH}$ values. In these experiments, the fluorescence images of the treated and control cells are taken while the cells are exposed to the solution containing pHrodo dextran. Therefore, the delivery of pHrodo Green $10 \mathrm{kDa}$ dextran is an indication of the endosomal pathway role in intracellular delivery. (c) Effect of flotillin-dependent endocytosis on the amount of delivery. (d) Exposure to continuous flow (shear stress only) and acoustic excitation on the amount of delivery for different 
exposure durations. Acoustically actuated cells exhibit significantly higher uptake compared to those exposed to shear stress only. Treatments are conducted on MDA-MB-231 cells using a 20 min continuous square-wave at $1 \mathrm{~W}$ input power. The mean intensities in $(\mathrm{a}-\mathrm{c})$ are normalized by the values of control experiments. The error bars indicate standard deviation of three independent experiments. 139

Figure E.5 Intracellular delivery of genes. Delivery results of (a) siRNA and (c) plasmid. Treatments are conducted on MDA-MB-231 cells using a 20 min continuous square-wave at $1 \mathrm{~W}$ input power. The mean intensities are normalized by the values of control experiments. The error bars indicate standard deviation of three independent experiments. 140

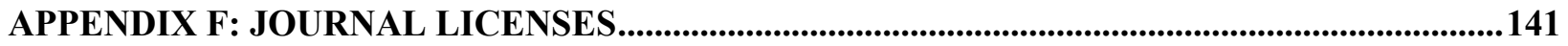

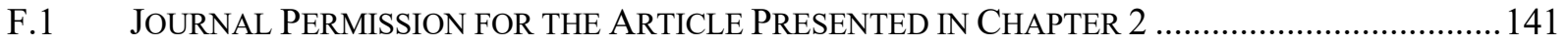

F.2 JOURNAL PERMiSSION FoR THE ARTICLE PRESENTED IN CHAPTER 3 .................................. 141

F.3 JOURNAL PERMISSION FOR THE ARTICLE PRESENTED IN CHAPTER 5 .................................. 143

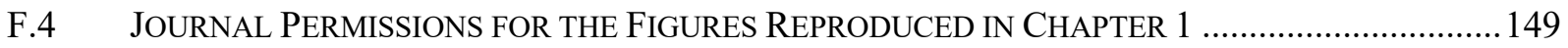




\section{Abbreviations}

\begin{tabular}{|c|c|}
\hline Abbreviation & Meaning \\
\hline $2 \mathrm{D}$ & 2 dimensional \\
\hline $3 \mathrm{D}$ & 3 dimensional \\
\hline AFM & Atomic force microscopy \\
\hline B-mode & Brightness-mode \\
\hline Bulk-NBs & Bulk-generated-nanobubbles \\
\hline $\mathrm{CD}$ & Cytochalasin D \\
\hline CVD & Chemical vapor deposition \\
\hline DI & Deionized \\
\hline DNA & Deoxyribonucleic acid \\
\hline DPPA & $\begin{array}{c}\text { (1,2-dipalmitoyl-sn-glycero-3-phosphate } \\
\text { (sodium salt)) }\end{array}$ \\
\hline DPPC & $\begin{array}{c}\text { (1,2-dipalmitoyl-sn-glycero-3- } \\
\text { phosphocholine) }\end{array}$ \\
\hline DPPE & $\begin{array}{c}\text { (1,2-dipalmitoyl-sn-glycero-3- } \\
\text { phosphoethanolamine) }\end{array}$ \\
\hline DSPE-mPEG (2000) & $\begin{array}{c}\text { (1,2-distearoyl-sn-glycero-3- } \\
\text { phosphoethanolamine-N- } \\
\text { [carboxy(polyethylene glycol)-2000] }\end{array}$ \\
\hline DSPE-PEG5F-15 & $\begin{array}{l}\text { 1,2-distearoyl-sn-glycero-3- } \\
\text { phosphoethanolamine-N-((polyethylene } \\
\text { glycol-5000)folate) (ammonium salt) }\end{array}$ \\
\hline DSPC & $\begin{array}{l}\text { 1,2-distearoyl-sn-glycero-3- } \\
\text { phosphocholine }\end{array}$ \\
\hline Echo-PIV & Echo particle image velocimetry \\
\hline FITC & Fluorescein isothiocyanate \\
\hline GFP & Green fluorescent protein \\
\hline HSC & Hele-Shaw cell \\
\hline MF-NBs & Microfluidically-generated-nanobubbles \\
\hline PBS & Phosphate-buffered saline \\
\hline PDMS & Polydimethylsiloxane \\
\hline PFA & Paraformaldehyde \\
\hline $\mathrm{PZT}$ & Lead Zirconate Titanate \\
\hline RMM & Resonant mass measurement \\
\hline RNA & Ribonucleic acid \\
\hline ROI & Region of interest \\
\hline SA & Specific aim \\
\hline SAW & Surface acoustic wave \\
\hline SDS & Sodium dodecyl sulfate \\
\hline SEM & Scanning electron microscope \\
\hline TEM & Transmission electron microscopy \\
\hline UV & Ultraviolet \\
\hline WGA & Wheat germ agglutinin \\
\hline
\end{tabular}




\section{Introduction}

A large portion of biomedical research is focused on diagnostics and intracellular delivery. Ultrasound, due to its non-invasive nature, has been suggested as a promising candidate for different diagnostic and therapeutic applications. For example, ultrasound elastography techniques are useful for conducting an early-stage diagnosis of liver diseases enabling a prompt therapeutic intervention before irreversible tissue damage occurs. ${ }^{1}$ Also, contrast-enhanced ultrasound exhibits great promise for imaging different tissues, especially for the diagnosis of primary tumors and metastasis, as well as, guiding cancer therapies. ${ }^{2}$ Gas-filled, encapsulated microbubbles have a significant role in many ultrasound biomedical applications due to their unique behavior in an acoustic field. Aside from imaging, payload-bearing microbubbles, for example, can be used in ultrasound-mediated targeted drug delivery applications. ${ }^{3}$

There is a strong demand for diagnostic tools capable of probing different biological properties of living organisms in both in vivo and in vitro settings. A variety of ultrasound-based techniques have been developed for the non-invasive visualization of living tissues. In medical ultrasound imaging, sound waves generated at a frequency above $20 \mathrm{kHz}$ are directed through tissues, where they can be reflected and attenuated as they travel through the tissue. The analysis of the backscattered waves is the basis of ultrasound imaging, which can provide crucial information about the anatomy and function of internal organs.

Ultrasound has also been used for enhancing intracellular delivery. For example, oscillations

of a microbubble in an acoustic field near a cell can impose localized forces on the plasma membrane and facilitate the delivery of extracellular material into the cytosol via a process called 
sonoporation. ${ }^{4}$ Different aspects involving the interaction of bubble oscillation and cells have been studied in the literature and include targeted cavitation, ${ }^{5}$ spatially controlled cavitation, ${ }^{6}$ and laserinduced cavitation. ${ }^{7}$

In the following sections of this chapter, diagnostic ultrasound and the use of microbubbles for ultrasound applications, as well as methods for intracellular delivery, are discussed. Next, Acoustofluidics is reviewed. The motivations and objectives of the proposed research are discussed next, followed by the thesis overview and the author's contribution in the context of collaborative publications.

\subsection{Diagnostic Ultrasound}

In medicine, a variety of tools and techniques are used to help diagnose a range of different diseases, dysfunctions, and ailments. Among those techniques, diagnostic ultrasound is a frequently used imaging procedure to monitor the functionality of different organs. In several biomedical ultrasound techniques, microbubbles and their interaction with sound waves are crucial to the effectiveness of the technique. A brief overview of bubble-mediated ultrasound techniques and the generation of bubbles is included in the following sections.

\subsubsection{Bubble-Mediated Ultrasound Techniques}

The emergence of microbubbles has expanded the diagnostic capabilities of ultrasound imaging of biofluid flows due to the high acoustic contrast generated when microbubbles are introduced into the flow. For example, the use of microbubble contrast agents has been shown to improve the accuracy of echocardiography for detecting the functional abnormalities of the heart. ${ }^{2}$ Evaluation of microvascular perfusion is another important application of microbubbles, which provide precise information on the volume and velocity of the blood. This information can be especially critical for the diagnosis of primary tumors and metastasis. ${ }^{2}$ 
The composition of the microbubble shell can also be engineered for targeting specific tissues. For this purpose, either the shell constituents bind to the target cell receptors, or the shell is functionalized with antibodies or ligands that bind to the target cell antigens.

Depending on the acoustic amplitude of the incident sound beam, a bubble can undergo stable or inertial cavitation. The stability of bubbles in such conditions is mainly governed by the transport properties of the gas core and shell composition. Figure 1.1 shows different scenarios of bubble-ultrasound interactions. There are a variety of commercially available microbubbles, which are developed for biomedical applications. Different gas cores, including air, nitrogen, and perfluorocarbon, and shell compositions, including albumin and lipid, have been used for making microbubbles with high intravascular stability. ${ }^{8}$

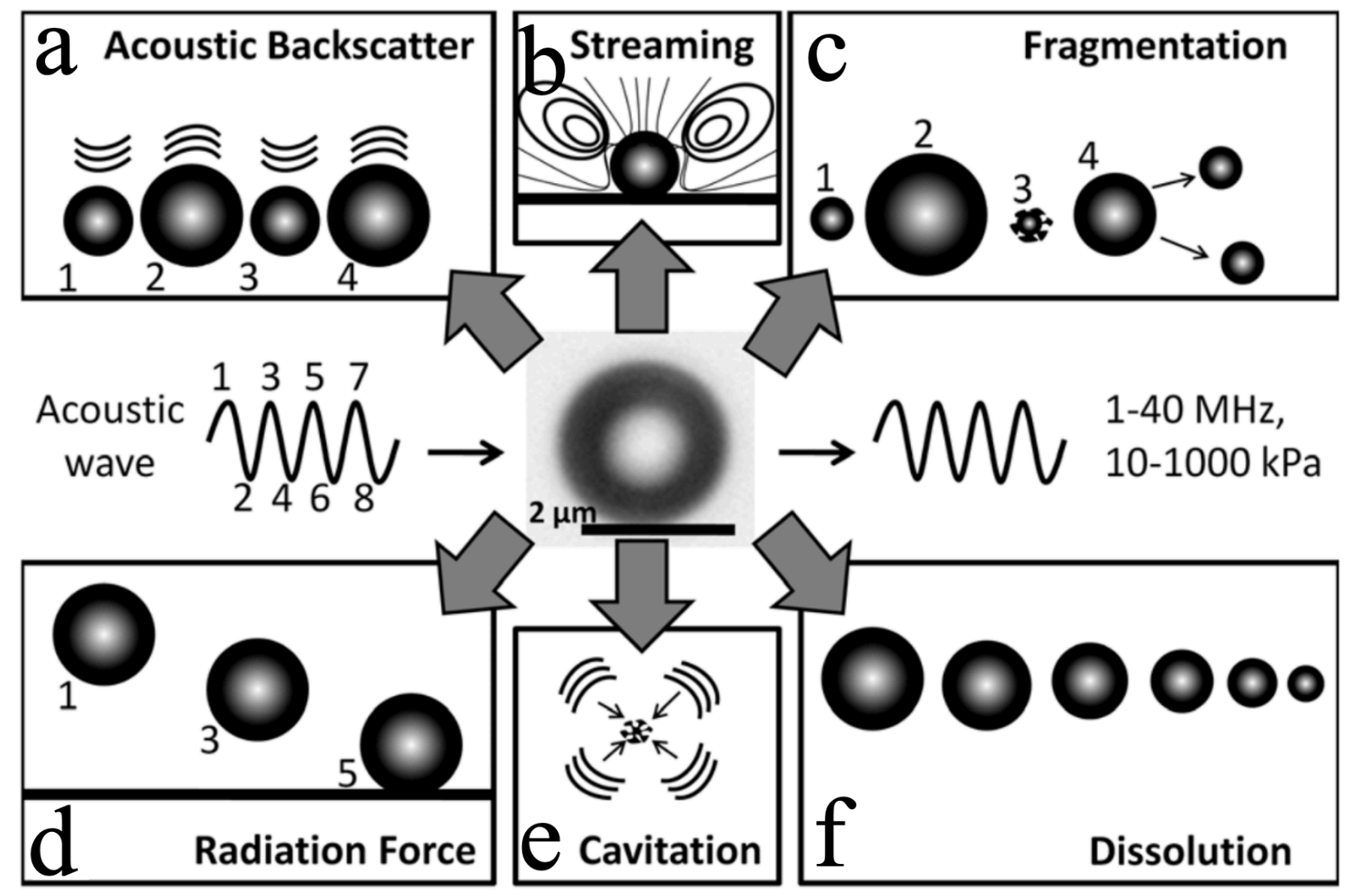

Figure 1.1 A variety of bubble-mediated effects used in ultrasound-based imaging and drug delivery techniques. (a) Detectable backscattered signals at moderate acoustic pressures. (b) The microstreaming effect due to stable cavitation of the bubble. (c) Fragmentation of a bubble into smaller daughter bubbles as a result of high acoustic pressures. (d) Radiation force directing the 
bubble away from the acoustic source or attracting it towards the other bubbles. (e) Inertial cavitation occurring as a result of high acoustic pressures. (f) Dissolution caused by acoustic pressures at the levels below the fragmentation threshold. Adapted from Sirsi and Borden. ${ }^{9}$

\subsubsection{Monodisperse Bubble Generation}

For medical applications, the bubble size needs to be below $10 \mu \mathrm{m}$ so that the bubble can safely pass through pulmonary and systemic capillaries. ${ }^{2,9}$ Also, the size distribution of the bubbles can greatly impact their acoustic response, ${ }^{10}$ as the resonance frequency of a bubble is related to its size. ${ }^{11}$ Therefore, sufficiently small bubbles with narrow size distribution and relatively long lifetime are critical for their function.

Hand-agitation and sonication of a surfactant solution, which are among the first methods developed for producing microbubbles, often require additional steps for separating bubbles with different size distributions. More recently, microfluidics has been used to generate bubbles with narrow size distributions. ${ }^{12-14}$ However, as the size of the microfluidically generated bubbles is directly related to the dimensions of the microchannel geometry, ${ }^{14}$ geometries with smaller orifices are needed in order to generate smaller bubbles. As a result, the generation of sub-10 $\mu \mathrm{m}$ diameter microbubbles is challenging, as it requires sophisticated micro- and nano-fabrication techniques.

More recently, the application of nanobubbles in various fields, such as surface cleaning ${ }^{15}$ and biomedical imaging, ${ }^{16}$ have been explored. The smaller size and longer lifetime are the two main features of nanobubbles, compared to microbubbles, for in vivo ultrasound imaging. ${ }^{16}$ Therefore, the generation of monodisperse nanobubbles is of great interest. ${ }^{17}$

\subsection{Intracellular Delivery}

It has always been a challenge to efficiently deliver different exogenous cargo materials into various cell types for different biomedical research and therapeutic applications. Depending on the application and the cells being investigated, different cargo materials are of interest for intracellular delivery. These include nucleic acids, proteins, peptides, membrane-impermeable 
drugs, molecular probes, and nanoparticles. Intracellular delivery techniques are used for different forms of gene editing and to modulate biological pathways of the cells. For instance, in cell-based therapies, various cellular gene manipulation approaches, such as RNA targeting, gene deletion, gene correction, and gene addition, are commonly used.

One of the most critical features of an intracellular delivery technique is that the effect of the materials, vectors, or physical forces used to facilitate the cellular uptake avoids permanently damaging the cell. Also, cell throughput (i.e., relatively low for rare cells, and high for a large number of cells) is another important characteristic of an intracellular delivery technique. In addition, an ideal technique should be universal across the cell types and the cargo molecules. The ability to control the dosage of delivery is also crucial to the efficiency of the technique. Moreover, having a low cost of production and operation can facilitate the clinical translation and large-scale manufacturing. ${ }^{18}$

The intracellular delivery strategies can be broadly categorized into carrier-based and membrane-disruption-based techniques. ${ }^{18}$ Carriers are various biochemical assemblies, which, can first package the cargo compound and protect it from the surroundings, and then can gain access to the intended compartment within the cell, where eventually the carrier can release the payload. Most carriers enter the cell through endocytosis. Different types of vectors, such as plasmids, cosmids, and viral vectors, can be used as carriers for the delivery of nucleic acids. Due to the high efficiency and specificity of viral vectors, they are the most clinically advanced delivery carriers. ${ }^{19}$ One main disadvantage of carrier-based systems is the limited number of consistent cargo materials and cell types. Due to the high variability of charge, hydrophobicity, size, and composition of cargo molecules, as well as, different receptors, surface interactions, and the endocytic activity of the cells, the combination of the cargo molecule and the carrier may not be stable during packaging, unpacking, and the delivery of sufficient quantities to the cells. ${ }^{18}$ Also, carrier-based techniques suffer from a low rate of delivery, unwanted toxic effects (e.g., genotoxicity in the case of viral vectors), and laborious and complex biochemistry, preparation, and material synthesis.

In contrast to the carrier-based techniques, the effectiveness of the membrane-disruptionbased techniques is dependent on the mechanism used for creating transient pores on the cell 
membrane. In many cases, it is a rapid process capable of delivering diverse materials into different cell types. These techniques include the application of mechanical forces (e.g., microinjection), ${ }^{20,21}$ electric forces (e.g., electroporation), ${ }^{22-24}$ shear forces (e.g., viscometer), ${ }^{25}$ thermal effects (e.g., heating-based systems), ${ }^{26}$ and optical-membrane interactions (e.g., laser-based photo-thermal systems). ${ }^{27,28}$ These approaches are summarized in Figure 1.2. Upon membrane disruption, which typically happens within seconds after the external force is applied onto the cell, nanoscale pores are created on the cell membrane facilitating the exchange of intracellular and extracellular contents. Depending on the pore size, the pore lifetime is on the order of minutes, after which the membrane repairs its pores and ultimately regains its integrity. The cytoplasmic recovery can then be started which can last up to several hours. ${ }^{18}$

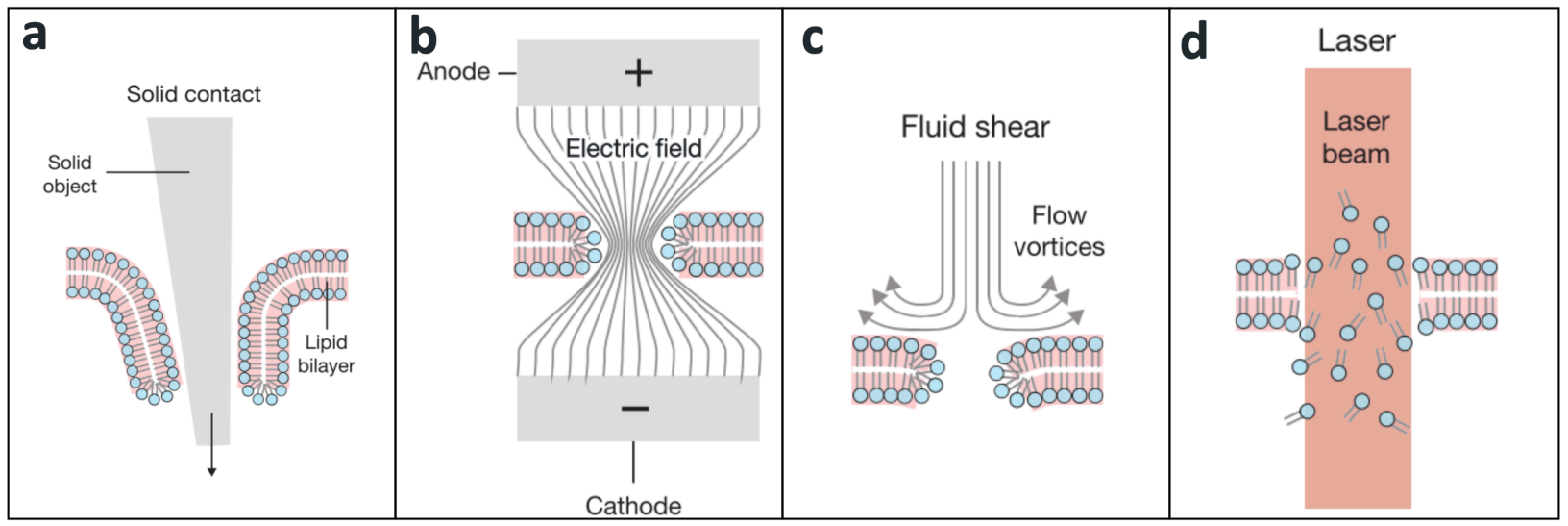

Figure 1.2 A schematic of different membrane-disruption-based strategies for intracellular delivery using (a) solid mechanical contact, (b) electric field, (c) shear stress, and (d) photo-thermal effect. Adapted from Stewart et al. ${ }^{18}$

Since the advent of the microinjection technique in $1911,{ }^{29}$ a wide range of different carrierbased and membrane-disruption-based strategies have been developed. For carrier-based techniques, hundreds of viral, lipid, polymer, and inorganic carriers, have been introduced mostly for transfection of nucleic acids..$^{18,30,31}$

Biological membranes are usually made of phospholipid bilayers, which consist of polar head-groups exposed to the aqueous environment and fatty acyl chains forming a hydrophobic core. ${ }^{32}$ The properties of the exogenous molecule (e.g., polarity and size), as well as the membrane 
properties (e.g., physical characteristics and composition), can affect the permeation through these membranes. $^{33}$

In should be noted that some of the membrane perturbation techniques can also be utilized for extracting intracellular materials. For instance, various cell lysis and tissue homogenization techniques can be used for extracting genomic and proteomic materials from the cells. ${ }^{34}$

\subsubsection{Mechanical Disruption Techniques}

Applying a mechanical force on a cell membrane, by exerting normal stress for causing penetration, or exerting shear stress for enhancing permeabilization, are the basis of several intracellular delivery techniques. Scraping and bead loading (e.g., gene gun or biolistics) of adherent cells, and repeated aspiration and expulsion through standard syringe needles for floating cells are among the classical techniques. ${ }^{35,36} \mathrm{~A}$ few of the most common mechanical techniques are reviewed here.

\subsubsection{Mechanical Penetration}

Using microneedles for direct injection of cargo molecules into the cell cytoplasm is the simplest mechanical way of membrane perturbation. More recent advances of these techniques are based on nano-scale tips, called nanoneedles (Figure 1.3), capable of penetrating the membrane such that the cargo molecule can either i) be released in the cytosol after the nanoneedle penetrates the membrane, or ii) reach the cytosol through a hollow nanoneedle (also called nanostraw), or iii) be diffused into the cytosol through holes caused by the nanoneedle removal. ${ }^{37-39}$ 


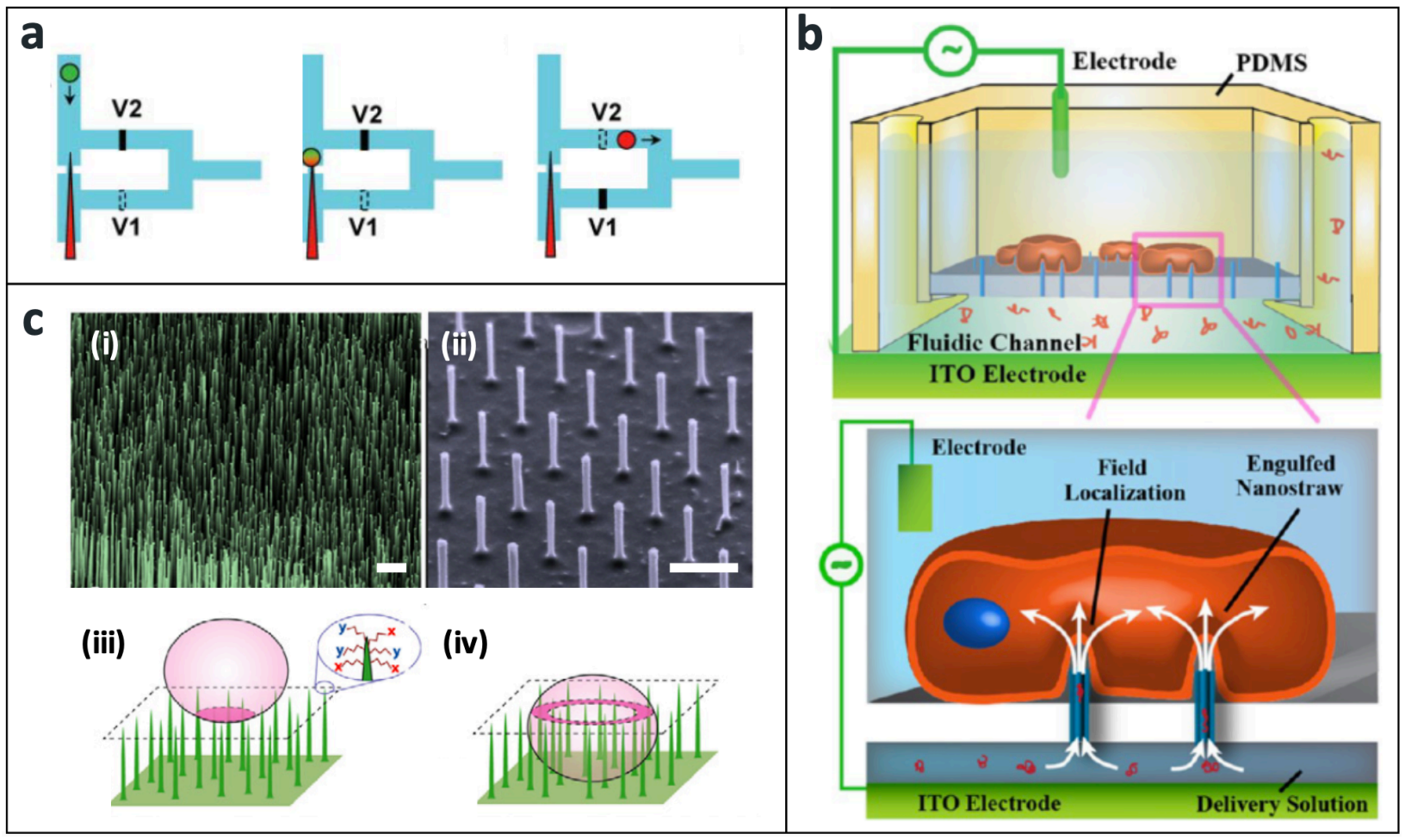

Figure 1.3 Mechanical penetration techniques for intracellular delivery. (a) The working principle of a microfluidic single-cell microinjection system where a cell is moved towards a fixed microneedle, while valve V1 is open and V2 is closed. Once the penetration occurred, the cell is moved to the outlet through valve V2 when V1 is closed. (b) A nanostraw-based device coupled with electroporation mechanism. The cargo molecules move into the cell cytoplasm through the nanostraws while the localized electric field facilitates this process. (c) SEM images of vertical silicon nanowires fabricated by (i) chemical vapor deposition (CVD) and (ii) reactive ion etching. The scale bars represent $1 \mu \mathrm{m}$. Schematic of (iii) early and (iv) late stages of cell penetration using the nanowires. Adapted from Shalek et al., ${ }^{38}$ Xie et al., ${ }^{39}$ Adamo and Jensen. ${ }^{40}$

Although the force needed for achieving an effective membrane penetration is estimated to be relatively low, i.e., $2 \mathrm{nN}$ per needle for a needle of $300 \mathrm{~nm}$ in diameter and $4 \mu \mathrm{m}$ in height, the requirement of a high-precession fabrication method is a significant challenge of the nanoneedles. ${ }^{18}$ 


\subsubsection{Shear-Based Permeabilization}

Shear stress can be created on a cell membrane by a solid object, fluid flow, or an acoustically driven microbubble. In macroscale bulk solutions, standard impellers operating at different speeds can cause a shearing environment on cells. ${ }^{41}$ However, in microfluidic cell squeezing platforms, shear stress is generated by imposing a rapid deformation to the cells when the cell suspension flows through constrictions with sizes smaller than the cells average diameter. ${ }^{42-44}$ The shear caused by the channel wall has shown to be able to transiently open the membrane pores so that a variety of cargo molecules, including proteins, nucleic acids, and nanomaterials, diffuse into the cytosol. ${ }^{43}$

Similarly, it has been shown that a high-velocity flow of a cell suspension through conical microfluidic channels with a diameter tapering from $300 \mu \mathrm{m}$ to $50 \mu \mathrm{m}$ can enhance the uptake of cell impermeant molecules into DU145 prostate cancer cells. ${ }^{45}$ Optimal viability of $80 \%$ and intracellular uptake to $30 \%$ of cells were reported when the cells experienced high shear rates for short durations. In addition, an uptake to $27 \%$ of cells was observed in a sample of monolayer adherent cells, which was exposed to a uniform shear of $140 \mathrm{dyn}^{-2}$ for $300 \mathrm{~ms}$, using a coneand-plate cell-shearing device.

Different viscometer designs, such as cone-plate ${ }^{46}$ and concentric cylinder ${ }^{47}$ configurations, are also capable of applying uniform flow-caused shear stress over apical membranes of a monolayer of adherent cells.

Hydrodynamic shear stress, $\tau_{h}$, caused by fluid flow inside a channel can be calculated as follows: ${ }^{48}$

$$
\tau_{h}=\frac{6 \mu_{L} Q}{w^{2} H}
$$


where, $Q$ is the flow rate, $\mu_{L}$ is the fluid viscosity, $w$ is the channel width, and $H$ is the height of the channel.

An alternative way of generating localized shear stress on the cell membrane is to actuate microbubbles near the target cell using ultrasound. It should be noted that the effect of a microbubble oscillation on its surrounding cell in an ultrasound field is not limited to shear, but can also include pushing, pulling, jetting, or translational motion (Figure 1.4). ${ }^{49}$ Contrary to electroporation, cells exposed to sonoporation can exhibit heterogeneous molecular uptake, which can widely vary for a population of cells. ${ }^{50}$

Actuation of a high density of microbubbles in a cell suspension can trigger inter-bubble interactions due to the secondary Bjerknes force and can ultimately result in bubble cluster formations, and bubble coalescence. ${ }^{51}$ Ultrasound enhanced microbubbles are thought to activate both the endocytosis and exocytosis pathways. ${ }^{52}$ Endocytosis consists of the formation of endocytic vesicles at the cell membrane which then move within the cell cytoplasm. Some of these vesicles may mature to degradative lysosomes. ${ }^{32,53,54}$ At high enough intensities, acoustic/ultrasound waves alone can also affect tissues/cells by sonodynamics (through sonochemistry) $)^{55}$ and sonophoresis (through shock waves). ${ }^{56}$

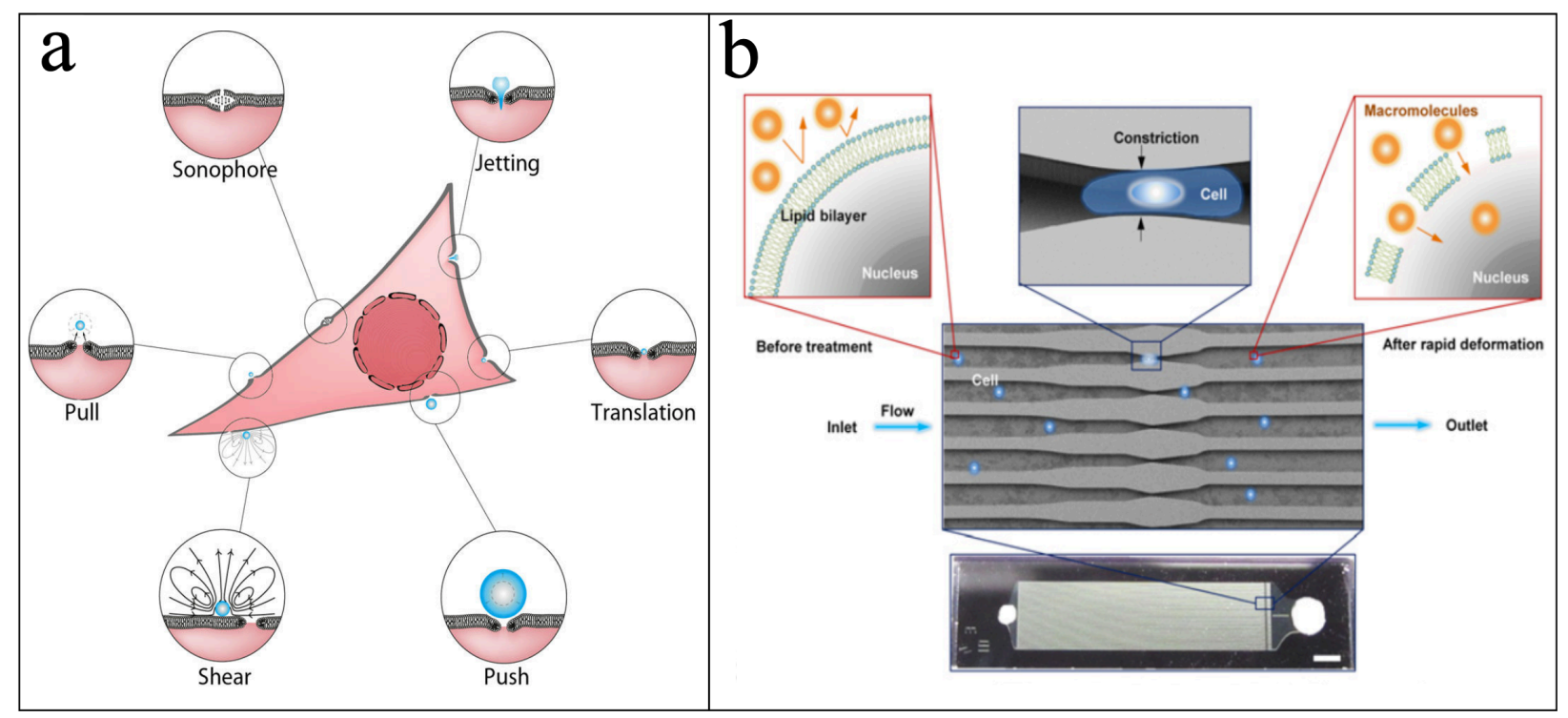

Figure 1.4 Examples of shear based permeabilization of the cell membrane. (a) A schematic of different aspects of sonoporation. Pulling, pushing, and microstreaming (i.e., shear) are caused by 
stable cavitation, whereas jetting is induced by inertial cavitation. The translational motion is due to the acoustic radiation force. Sonophoresis is induced mainly by shock waves as a result of lowfrequency ultrasound. (c) A shear-mediated intracellular delivery based on the rapid deformation of cells when they pass through a microfluidic constriction. Adapted from Sharei et al. ${ }^{43}$ and Kotopoulis et al. ${ }^{57}$

Shear stress can affect the size and morphology of the cells, ${ }^{58}$ can create pores in the cell membrane, and can activate biochemical pathways that result in gene expression, membrane permeability changes, and altered metabolism. ${ }^{47,59,60}$ Shear stress can also activate pathways that transiently increase fluid-phase endocytosis. Macropinocytosis is a fluid-phase endocytosis mechanism for non-specific uptake of extracellular molecules by vesicular endocytosis. ${ }^{61,62}$

Steady shear stress imposed on endothelial cells can align the cells with their longitudinal axis parallel to the direction of flow, which in turn can decrease the shear stress on the endothelial cells. ${ }^{60,63}$ Since excessive shear forces can cause irreversible damage to the cell membrane, several medium additives have been suggested for the intracellular delivery techniques, which can diminish the harmful effects of shear force on the cells. These include methylcellulose, ${ }^{64}$ serum, polyethylene glycol, ${ }^{65}$ dextran, and Pluronic F-68. ${ }^{66,67}$ The additives can increase the robustness of the cell membrane to physical forces and can provide protection against the rupture of gas-liquid interfaces. ${ }^{67}$

\subsection{Acoustofluidics}

Acoustofluidics is a relatively new field of research and involves fluid mechanics, acoustics, and micro/nanotechnology. In this section, the physics of acoustics, the propagation of acoustic waves through microfluidic devices, and the interaction of acoustic waves and bubbles (or any elastic material) are discussed.

Ultrasound transducers with a frequency range of 1-20 MHz are commonly used in clinical imaging applications. Due to the nature of mechanical waves, propagation of ultrasound in liquids 
is longitudinal, while in solids (e.g., bones) and elastic materials, the waves can be both longitudinal and transverse. ${ }^{68}$

For a harmonic plane wave propagating through a medium, the acoustic pressure, $p$, experienced by the particles of the medium can be written as: ${ }^{68}$

$$
p=\rho c u,
$$

where, $\rho, c$, and $u$ are the medium density, speed of sound, and the particle velocity, respectively.

When an acoustic wave encounters the interface of two media, depending on the acoustic properties of the media, a portion of the wave may be transmitted, and a portion may be reflected. The ratio of the transmitted wave, $T$, and reflected wave, $R$, to an incident acoustic wave normal to the interface can be expressed as: ${ }^{68}$

$$
\begin{aligned}
& T=\frac{2}{\left(1+\frac{Z_{1}}{Z_{2}}\right)}, \\
& R=\frac{\left(Z_{2}-Z_{1}\right)}{\left(Z_{2}+Z_{1}\right)},
\end{aligned}
$$

where $Z_{1}$ and $Z_{2}$ are the acoustic impedances of the two media and can be defined as $Z=\rho c$.

The acoustic intensity, $I$, and energy exposure (spatial peak), $E$, for a harmonic wave, can be written as: ${ }^{68,69}$ 


$$
\begin{aligned}
& I=\frac{P^{2}}{2 \rho c}, \\
& E=I \cdot t,
\end{aligned}
$$

where, $P$ and $t$ are the pressure amplitude and time, respectively. The intensity of an ultrasound wave while propagating through a medium in the direction of $x$ decreases due to absorption in a homogenous medium. In an inhomogeneous medium, the intensity decreases due to both absorption and scattering. The reduction in intensity can be expressed as: ${ }^{68}$

$$
\begin{gathered}
I=I_{0} e^{-2 \alpha x}, \\
\alpha=\alpha_{a}+\alpha_{s},
\end{gathered}
$$

where, $\alpha, \alpha_{a}$ and $\alpha_{s}$ are the total attenuation coefficient, absorption attenuation coefficient, and scattering attenuation coefficient, respectively. $I_{0}$ is the initial intensity of the wave.

\subsubsection{Surface Acoustic Wave (SAW)}

In acoustofluidics, sound waves are generated either as bulk waves or surface waves. A common type of surface acoustic waves (SAW) is Rayleigh waves, which are a combination of longitudinal and transverse waves. Rayleigh waves decay exponentially with the material depth and thus travel only on the surface of a medium. Due to the nature of surface waves, the particles of the medium experience a combination of longitudinal and transverse motions resulting in an elliptical displacement. ${ }^{70}$ In many microfluidic applications, Rayleigh waves can be generated and transmitted on the surface of the device substrate. ${ }^{71}$ A more general form of Rayleigh wave is referred to as the guided wave, where the bulk waves satisfy specific boundary conditions. The equations governing Rayleigh waves and bulk waves are similar, with one difference: the Rayleigh solution satisfies the boundary condition on the surface of an elastic object. ${ }^{71}$ The analysis of a 
specific guided wave through a thin plate, which has a thickness smaller than, or comparable to, the sound wavelength, was first solved by H. Lamb about a century ago. The solution for such a system results in waves called Lamb waves. ${ }^{72}$ At low frequencies, two modes of Lamb waves are possible, i.e., symmetric and antisymmetric modes. ${ }^{73}$

When a Rayleigh surface acoustic wave travels on a surface in contact with a liquid, it can leak energy into the fluid in the form of acoustic waves, which in turn, generates a time-averaged (or mean) flow in the fluid. ${ }^{74}$ In general, acoustic streaming can be generated due to the dissipation of energy, either through the liquid bulk, which is called interior streaming, or confined in thin boundary layers, which is called boundary streaming. ${ }^{74}$ The angle at which the Rayleigh surface acoustic wave leaks into the liquid is called Rayleigh angle, $\theta_{R}$ (Figure 1.5), and can be determined as: $:^{75}$

$$
\theta_{R}=\arcsin \frac{v_{\text {fluid }}}{v_{R S A W}}
$$

where, $v_{\text {fluid }}$ is the speed of sound in the fluid, and $v_{R S A W}$ is the velocity of the Rayleigh surface acoustic wave.

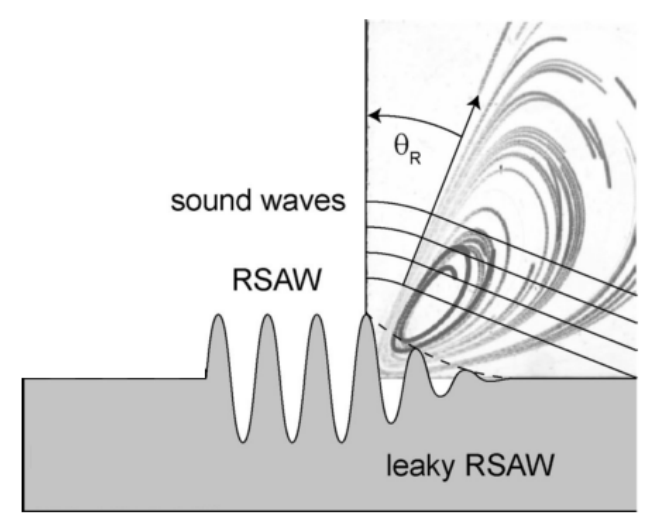

Figure 1.5 Acoustic streaming flow generated by the radiated sound waves at the Rayleigh angle into the fluid. Adapted from Frommelt et al..$^{75}$ C 2008 IEEE. 
The physics of the acoustic waves, especially SAWs, which are reviewed in this section, can predict the formation of microstreaming flow within microfluidic channels. Microvortices generated by these streaming flows can drag particles (e.g., cells) suspended in the fluid into motion, causing them to rotate.

\subsubsection{Microstreaming}

When an acoustic wave actuates a microbubble (or a highly deformable object) in an acoustofluidic device, an oscillatory viscous boundary layer is created adjacent to the gas-liquid interface inside the liquid, due to the viscous dissipation of acoustic energy (Figure 1.6). The thickness of this oscillatory boundary layer, $\delta$, is expressed as: ${ }^{74,76-79}$

$$
\delta=\sqrt{\frac{\mu}{\rho \pi f}},
$$

where, $\mu, \rho$, and $f$ are medium viscosity, medium density, and the oscillation frequency.

The shear stress, $\tau$, caused by the velocity gradient across the boundary layer near an oscillating bubble can be written as: ${ }^{76,77,80}$

$$
\tau=2 \varepsilon R_{0} \sqrt{\pi^{3} \rho f^{3} \mu}
$$

where, $\varepsilon$ is the relative displacement amplitude of a bubble with a radius (at rest) of $R_{0}$. 


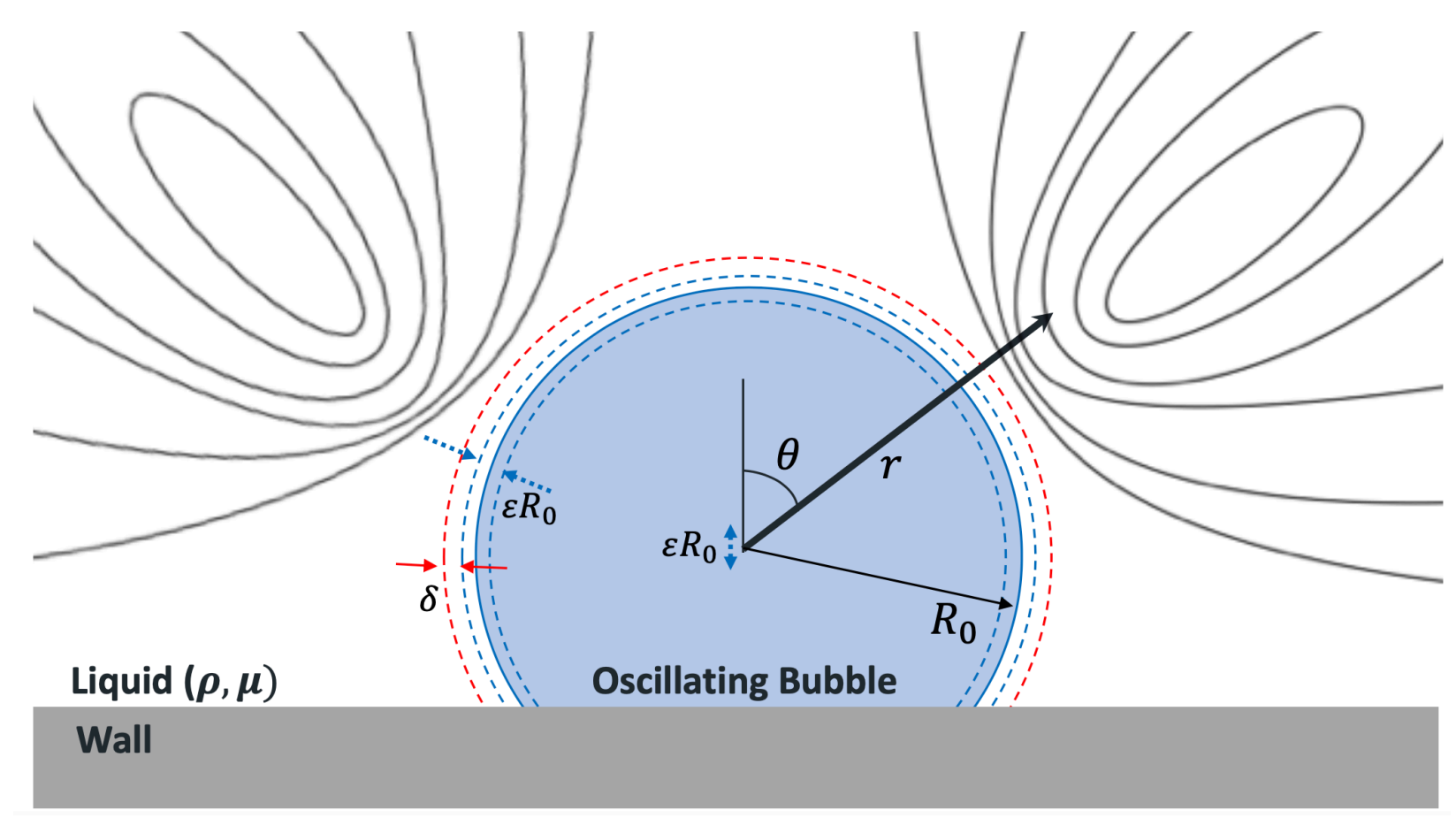

Figure 1.6 Schematics of microstreaming flows generated next to an oscillatory bubble with an initial radius $R_{0}$. A steady streaming flow is generated outside the boundary layer due to the recirculating flows within the boundary layer. The amplitude of translational and volume oscillations is equal to $\varepsilon R_{0}$. The dashed red line represents the boundary layer, which has a thickness of $\delta$. $\mathrm{r}$ and $\theta$ are the polar coordinates.

The normal stress, $\sigma$, caused by an oscillating microbubble can be estimated using Euler's equation as follows: ${ }^{81}$

$$
\sigma=4 \pi^{2} f^{2} \rho R_{0}^{2} \varepsilon .
$$

The formation of an oscillatory viscous boundary layer can cause a net streaming flow (also called microvortices) in the bulk of the liquid, which is called acoustic streaming or microstreaming. For example, when the volume of a bubble, which is attached to a horizontal solid surface inside a liquid, changes periodically while its bottom remains fixed to the surface, the bubble shows both translational (due to the up-and-down motion of its center of mass) and volume 
(due to the changes in bubble volume) oscillations. The stream function, $\psi$, of the steady-state flow generated around such a bubble can be estimated in polar coordinates as follows: ${ }^{82,83}$

$$
\psi \approx-6 \varepsilon^{2} R_{0}^{3} \pi f \sin (\Delta \phi)\left(\frac{R_{0}}{r}\right) \cos ^{2} \theta \sin ^{2} \theta
$$

where, $\Delta \phi$ represents the phase shift between volume and translational oscillations. $r$ and $\theta$ are the distance to the bubble center and the angle with the axis of translation (i.e., gravity direction), respectively.

Since solving the compressible Navier-Stokes equations, which govern both the liquid motion and the acoustic wave propagation, is computationally expensive, a perturbation approach can be used. One can assume that the acoustic wave constitutes tiny perturbations in the fields of pressure, density, and velocity within the liquid: ${ }^{70,84}$

$$
\begin{aligned}
& \rho=\rho_{0}+\epsilon \rho_{1}+\epsilon^{2} \rho_{2}+\cdots, \\
& p=p_{0}+\epsilon p_{1}+\epsilon^{2} p_{2}+\cdots, \\
& v=v_{0}+\epsilon v_{1}+\epsilon^{2} v_{2}+\cdots
\end{aligned}
$$

By incorporating the above equations into the Navier-Stokes and continuity equations, the acoustic wave propagation can be characterized. For a system with a liquid initially at rest, the first-order equations can be written as: ${ }^{70,79,85,86}$

$$
\frac{\partial \rho_{1}}{\partial t}+\rho_{0} \boldsymbol{\nabla} \cdot \boldsymbol{v}_{\mathbf{1}}=0
$$




$$
\rho_{0} \frac{\partial v_{1}}{\partial t}=-\nabla p_{1}+\mu \nabla^{2} v_{1}+\left(\frac{1}{3} \mu+\mu_{B}\right) \nabla\left(\nabla \cdot v_{1}\right)
$$

and by time-averaging the second order equations over one period of oscillation, we will have:

$$
\begin{gathered}
\left\langle\frac{\partial \rho_{2}}{\partial t}\right\rangle+\rho_{0} \boldsymbol{\nabla} \cdot\left\langle\boldsymbol{v}_{\mathbf{2}}\right\rangle+\boldsymbol{\nabla} \cdot\left\langle\rho_{1} \boldsymbol{v}_{\mathbf{1}}\right\rangle=0 \\
\rho_{0}\left\langle\frac{\partial \boldsymbol{v}_{\mathbf{2}}}{\partial t}\right\rangle+\rho_{1}\left\langle\frac{\partial \boldsymbol{v}_{\mathbf{1}}}{\partial t}\right\rangle+\rho_{0}\left\langle\left(\boldsymbol{v}_{\mathbf{1}} \cdot \boldsymbol{\nabla}\right) \boldsymbol{v}_{\mathbf{1}}\right\rangle \\
=-\boldsymbol{\nabla}\left\langle p_{2}\right\rangle+\mu \nabla^{2}\left\langle\boldsymbol{v}_{\mathbf{2}}\right\rangle+\left(\frac{1}{3} \mu+\mu_{B}\right) \boldsymbol{\nabla}\left(\boldsymbol{\nabla} \cdot\left\langle\boldsymbol{v}_{2}\right\rangle\right) .
\end{gathered}
$$

The solutions to the first-order equations and the equation of state $p_{1}=c^{2} \rho_{1}$, give rise to the acoustic pressure and the velocity within the streaming flow. The non-zero time-averaged velocity of the second-order perturbation equation gives the acoustic streaming flow representing the absorption of acoustic energy and momentum. ${ }^{84,85}$

When solving Eqs. (1.11) and (1.12) in a microfluidic device, different pressure boundary conditions are applied at the channel walls. A general boundary condition, typically referred to as lossy-wall condition (e.g., Polydimethylsiloxane (PDMS)/liquid interface), is modeled as: ${ }^{70,84}$

$$
\text { n. } \nabla p_{1}=i \frac{\omega \rho_{0}}{Z_{w}} p_{1}
$$

where, $Z_{w}, \omega$, and $\boldsymbol{n}$ are the acoustic impedance of the wall material, angular frequency, and the unit vector normal to the boundary, respectively. If the boundary is assumed to be soft (e.g., air), and therefore, $Z_{w} \approx 0$, then Eq. (1.12) can be reduced to $p_{1}=0$. In contrast, for a hard-wall (i.e., $\left.Z_{w} \rightarrow \infty\right)$, the boundary condition can be written as $\boldsymbol{n} \cdot \nabla p_{1}=0$. 
The time-averaged secondary radiation force, $\boldsymbol{F}_{\boldsymbol{R}}$, exerted on a spherical particle in an acoustic field caused by an oscillating microbubble can be expressed as: ${ }^{87,88}$

$$
\boldsymbol{F}_{\boldsymbol{R}}=\frac{4}{3} \pi \rho_{p} \phi_{c} \cdot \frac{R_{b}^{4} R_{p}^{3}}{d^{5}} \omega^{2} \varepsilon^{2},
$$

where, $\rho_{p}, R_{b}, R_{p}$, and $d$ are particle density, microbubble radius, particle radius, and distance between the center of the bubble and the particle, respectively. The acoustic contrast factor, $\phi_{c}$, can be defined as follows:

$$
\phi_{c}=3 \frac{\left(\rho_{p}-\rho\right)}{\left(2 \rho_{p}+\rho\right)} .
$$

The velocity, $\boldsymbol{v}_{\boldsymbol{s}}$, of microstreaming flow generated due to an oscillating microbubble can be written as follows: ${ }^{87,88}$

$$
v_{s}=\frac{R_{b}^{4}}{d^{5}} \omega \varepsilon^{2} .
$$

Stokes' drag theory estimates the force, $\boldsymbol{F}_{\boldsymbol{D}}$, exerted on particles due to the velocity difference of particle and the streaming flow: 88,89

$$
\boldsymbol{F}_{\boldsymbol{D}}=6 \pi \mu R_{p}\left(\boldsymbol{v}_{\boldsymbol{s}}-\boldsymbol{v}_{p}\right),
$$

where, $\boldsymbol{v}_{p}$ is particle velocity. 


\subsection{Motivation and Objectives}

\subsubsection{Microfluidic Approaches for Bubble Generation}

The generation of sufficiently small microbubbles with a relatively long lifetime, narrow size distribution, and high production rate is critical for the effectiveness of ultrasound techniques. Conventional microbubble generation techniques, such as sonication and mechanical agitation, results in poly-disperse populations, and therefore, it requires additional centrifugation and separation steps for achieving mono-disperse populations. Microfluidic techniques have enabled a high degree of controllability on the size and production rate of the bubbles. However, as the size of the microfluidically generated bubbles is directly related to the dimensions of the microchannel geometry, ${ }^{14}$ smaller geometries are needed in order to generate smaller bubbles. As a result, the generation of sub-10 $\mu \mathrm{m}$ diameter microbubbles is challenging, as it requires sophisticated microfabrication techniques.

To overcome these challenges, two microfluidic approaches are proposed here. In the first approach, the size of bubbles is precisely controlled as they are shrunk using a vacuum system in a microfluidic device. This technique is based on applying vacuum pressure in a PDMS based microfluidic chip to deplete air from the liquid-filled microchannels so that originally large bubbles shrink to smaller size bubbles. ${ }^{90}$ In the second approach, an expansion-mediated bubble breakup method is presented. Originally large bubbles can be split either symmetrically or asymmetrically as they flow through a microfluidic expansion region yielding bi- or tri-disperse populations, respectively. By controlling the fluid properties and flow speed, different breakup regimes can be achieved.

\subsubsection{Acoustofluidics for Intracellular Delivery}

Despite the advantages of membrane-disruption-based strategies over carrier-based systems, there are still some limitations in the current membrane-disruption-based techniques which need to be addressed. Some of these modalities can cause excessive damage to biological compounds

within the cell, denature protein molecules, or breakdown the lipid membrane. Some techniques, such as microinjection, are low-throughput since cells need to be injected one at a time. ${ }^{18,91}$ Some 
other high-throughput systems such as electroporation lack single-cell precision. ${ }^{22,91}$ In a recently reported microfluidic-based high-throughput technique, ${ }^{42,43,91}$ the cell suspension needs to pass through multiple constriction areas, which enhance the cytosolic delivery to the squeezed cells. Due to the high pressures required for flowing cells through the constrictions, clamping tools (e.g., stainless-steel holders) are required to avoid leakage. Also, since the constriction size is smaller than the cell size, channel clogging is inevitable. In addition, to account for the size variations of different cell lines, channel constrictions should be custom-designed according to the size of the cells being treated. ${ }^{42,43,91}$

Given the issues mentioned above, a novel acoustofluidic strategy is proposed for enhancing cellular uptake by exciting cells using acoustic waves. To better control cell positioning, acoustic parameters, and fluid flow parameters, the acoustic field is coupled to a microfluidic device. This strategy does not require sophisticated fabrication techniques, complex working equipment, and precise alignment, in contrast to the other microfluidic techniques (e.g., constriction-based technique). ${ }^{42,43,91-93}$ Also, the formation of aggregated cells is reduced compared to other techniques that use highly localized external fields (e.g., electrical/thermal techniques). In addition, this acoustofluidic strategy enables a high-throughput and high viability approach with dosage control for intracellular delivery.

\subsubsection{Research Objectives}

The following two research objectives are presented:

- To develop microfluidic approaches based on the shrinkage and breakup of bubbles for the production of monodisperse micro- and nano-bubbles suitable for different ultrasound applications.

- To develop acoustofluidic strategies for intracellular delivery.

\subsubsection{Microfluidic approaches for the generation of monodisperse bubbles}

To reach the first objective, the following questions need to be answered: 
- What are the parameters governing the bubble shrinkage?

- How can the final size of a shrinking microbubble be controlled?

- How can bubbles be split into smaller bubbles using microfluidics in order to make microbubbles at a high production rate?

- How can fluid properties and flow rates be adjusted to achieve microbubbles with different size distributions?

Typically, microbubbles are generated using a variety of microfluidic geometries. The final size of microbubbles is mainly dictated by the dimensions of the geometry, and as a result, generating bubbles on the order of microns (or sub-microns) with a narrow size distribution demands a highly precise micro- and nanofabrication technique, which can be costly and sophisticated. To overcome this challenge, it will be shown that initially large size bubbles can be shrunk or broken into smaller pieces in the flow so that smaller bubbles with a narrow size distribution can be collected. Sub-micron bubbles generated with such techniques can then be used for ultrasound imaging and drug delivery.

The proposed research for the microfluidic approaches for the generation of monodisperse bubbles has the following specific aims (SA):

- SA1: To characterize the dynamics of bubble size variations in a vacuum-assisted microfluidic system so that the microbubble size can be controlled as a function of design parameters.

- $\quad$ SA2: To design and characterize a high-throughput microfluidic device for breaking bubbles into smaller daughter bubbles with high controllability and to develop a dimensional analysis for the characterization of breakup regimes.

- $\quad$ SA3: To employ the results of SA1 for generating sub-micron bubbles for ultrasound imaging. 


\subsubsection{Acoustofluidics for intracellular delivery}

To reach the second objective, the following questions need to be answered:

- How does an adherent cell respond to an acoustic wave?

- Under an acoustic excitation, can the cell stiffness be involved in the cellular response?

- Is there a significant dependence between intracellular delivery and cells being actuated by acoustics?

- Can the actuation parameters be used for controlling the dosage of intracellular delivery?

- In comparison to the existing intracellular delivery methods, can this platform enhance the throughput?

An acoustofluidic platform enabling a controlled acoustic excitation of adherent cells is presented. The cell-induced microstreaming effect and its dependence on the cellular stiffness are studied. This platform is also shown to facilitate the cytosolic delivery, as the cargo material is taken up by the cells as a result of acoustic excitation and shear forces imposed by microstreaming effect. The previously-reported intracellular delivery techniques suffer from low viability and efficiency. This acoustofluidic strategy can enable high controllability and scalability. Since unfocused acoustic waves are used, no sophisticated equipment or alignment is required. It can also be combined with other carrier-based techniques for obtaining higher delivery efficiencies. This platform can eventually be coupled with other biological and analytical techniques in a labon-a-chip device.

The proposed research in the use of acoustofluidics for intracellular delivery has the following specific aims:

- SA1: To design, fabricate, and test an acoustofluidic platform for oscillating cells in a microfluidic channel, and study the cellular response to the acoustic excitation. 
- SA2: To facilitate the delivery of cargo compounds (e.g., dextran with different molecular weights) into cells using this acoustofluidic platform, and to control the amount of delivery based on the acoustic excitation parameters.

\subsection{Overview of the Dissertation}

In this thesis, novel methodologies for a) the generation of microbubbles for different ultrasound applications and b) intracellular delivery using acoustofluidics are presented. Due to its high controllability in micro-scale fluid-related phenomena, microfluidics has been employed to develop these methodologies.

Each of the Chapters 2-6 is written in the format of an article, which either has been published or is currently in preparation for submission to a journal. The permissions obtained from the journals for the published articles are included in Appendix F.

The next three chapters, i.e., Chapters $2-4$, are focused on developing new microfluidic methodologies of making microbubbles and nanobubbles. In Chapters 5 and 6, an acoustofluidic technique for intracellular delivery is discussed. Chapter 7 has conclusions and future work.

In Chapter 2, a theoretical and experimental framework is presented for characterizing microbubbles made with various lipid concentrations flowing in solutions that have different interfacial tensions. A vacuum system is coupled with a bubble generation system to actively control the final size of microbubbles as they flow through a gas permeable microfluidic device. The changes in the size of bubbles caused by applying vacuum pressure are monitored for various vacuum pressures. The physics governing the shrinkage mechanism is then described by a mathematical model that predicts the resulting bubble sizes and elucidates the dominant parameters controlling bubble sizes.

In Chapter 3, a novel microfluidic platform for breaking originally large bubbles into smaller sized daughter bubbles is introduced. The breakup occurs as a result of bubbles flowing through a microchannel equipped with an expansion region, where the channel width increases at $90^{\circ}$. In this 
flow regime, bubbles generated at a cross-junction periodically breakup into smaller daughter bubbles, upon entering the expansion region. In addition to the Capillary number, which is previously shown to govern the dispersion breakup in such geometries, we find that, at a highinertia regime, the Weber number also plays a significant role in specifying the transition from non-breakup to breakup regimes. Furthermore, we identify different periodic breakup modes, for example, symmetric and asymmetric breakup, which are dictated by the Ohnesorge number. Without modifying the geometry and by only tuning several dimensionless parameters related to the fluid flow, we show that a microchannel expansion region can produce mono-, bi-, or tridisperse microbubble populations.

In Chapter 4, the bubble shrinkage technique is further explored by making bubbles with a mixture of two different gasses as their gas core: A low molecular weight gas (e.g., nitrogen) mixed with a high molecular weight gas (e.g., octafluoropropane). Due to its higher diffusion and permeability coefficients, the diffusion timescale of the low molecular weight gas from the bubble to the liquid bulk can be on orders of magnitude shorter. We show that by controlling the mixing ratio of the two gasses, microbubbles with different final sizes, including sub-micron sized bubbles, can be produced.

In Chapter 5, a novel acoustofluidic platform is introduced for actuating adherent cells using acoustic waves. We report new observations that individual cells are able to induce microstreaming flow when they are excited by a controlled acoustic excitation in vitro. In our acoustofluidic platform, an acoustic wave excites adherent cells, forcing them to oscillate inside a microfluidic channel. We investigate the cell-induced microstreaming by monitoring flow tracers around the cell, while the structure and extracellular environment of the cell are altered using different chemicals. Our observations suggest that the maximum streaming flow induced by adherent cells can reach velocities on the order of $\mathrm{mm} \mathrm{s}^{-1}$, and this maximum velocity is primarily governed by the overall cell stiffness. Therefore, we propose that such cell-induced microstreaming measurements, including flow pattern and velocity magnitude, may be used as label-free proxies of cellular mechanical properties, such as stiffness. As the microstreaming generates shear stress on the cells, the newly discovered phenomenon could be used for intracellular delivery. 
In Chapter 6, we demonstrate that our acoustofluidic platform can facilitate the cellular uptake of different size cargo materials. Strong oscillations of cells and the microstreaming effect inside a microfluidic channel cause the cells to uptake different size (e.g., 3-500 kDa) cargo materials, mainly through endocytic pathways. High viability ( $\geq 91 \%)$, versatility across different cargo materials and various cell lines, and scalability to hundreds of thousands of cells per treatment are among the unique features of this acoustofluidic strategy.

In Chapter 7, the overall results achieved throughout this research are summarized, the author's publications are listed, and potential future research directions are discussed.

The appendix has a collection of additional information related to the chapters.

\subsection{Author's Contribution in the Context of Collaboration}

The author has made a substantial contribution to all the techniques and methodologies presented in this thesis. The amount of contribution to the articles, made by each author, excluding the supervisors, are summarized below.

For the article presented in Chapter 2, the author wrote the manuscript, developed the mathematical model, designed and conducted the experiments, collected and analyzed data, and fabricated the devices. V. Gnyawali helped with running the first set of experiments. I. Griffiths helped with solving the final differential equation analytically and commenting on the manuscript. R. Karshafian helped with providing equipment and supervision.

For the article presented in Chapter 3, the author wrote the majority of the manuscript, developed the dimensional modeling, designed the experiments, conducted the data analysis, and fabricated the devices. J. Xu performed the majority of the experiments, and collected the majority of the experimental data, and helped with discussing the results and writing the manuscript.

For the article presented in Chapter 4, the author designed and conducted the proof-ofconcept experiments, and fabricated the microfluidic devices, before J. Xu took the lead for the 
rest of the project. Throughout the project, the author actively helped with discussing the results and commenting on the manuscript. J. Xu led the experiments and wrote the manuscript. Y. Wang and $\mathrm{X}$. He performed the in vitro and in vivo experiments. L. Kerr helped with the bubble shrinkage experiments and resonant mass measurements. A. Darbandi performed the TEM characterizations. D. Yuen contributed to the design of the in vivo experiments and provided animal samples. A. C. de Leon and A. A. Exner assisted in developing the lipid solution protocols and bulk nanobubble generation experiments.

For the article presented in Chapter 5, the author wrote the manuscript and designed, fabricated, and tested the devices. Other than the atomic force microscopy measurements, the author designed and conducted all the experiments and collected and analyzed data. S. AppakBaskoy helped with providing chemicals, staining protocols, discussions, and comments on the manuscript. M. Ezzo and B. Hinz conducted and analyzed atomic force microscopy measurements.

For the article presented in Chapter 6, the author wrote the majority of the manuscript, designed, fabricated, and tested the devices, conducted all experiments, and collected and analyzed data. S. Appak-Baskoy helped with providing chemicals and optimized protocols for conducting staining and gene delivery, discussions, and writing the manuscript. J. Abousawan and C. Antonescu provided a cell line and discussions. I. Coe provided equipment, discussion, and supervision. 


\section{Bubble Size Control in Microfluidic Channels}

The work presented in this chapter is based on the following article,${ }^{94}$ which is published in a peer-reviewed journal Soft Matter, and is reproduced here with permission from The Royal Society of Chemistry.

Salari, A., Gnyawali, V., Griffiths, I.M., Karshafian, R., Kolios, M.C., and Tsai, S.S.H. Shrinking microbubbles with microfluidics: Mathematical modelling to control microbubble sizes. Soft Matter 13, 8796-8806 (2017).

\section{Author's Contribution}

For the article presented here, the author wrote the manuscript, developed the mathematical model, designed and conducted the experiments, collected and analyzed data, and fabricated the devices. V. Gnyawali helped with running the first set of experiments. I. Griffiths helped with solving the final differential equation analytically and commenting on the manuscript. R. Karshafian helped with providing equipment and supervision.

\subsection{Introduction}

Microbubbles are currently used as ultrasound contrast agents, ${ }^{95}$ and also increasingly applied to drug delivery systems, ${ }^{96}$ as therapy agents, ${ }^{97}$ and as oxygen-transfer agents. ${ }^{98}$ In 
ultrasound imaging, microbubbles help provide important information about tissues, blood vessels, and drug pathways in the body., 2,99 For example, in cancer diagnostics, tumor detection using traditional ultrasound is challenging because tumors and the surrounding tissues have similar acoustic impedance. Therefore, microbubbles are employed to increase the ultrasound contrast. The bubble gas-liquid interface has a high reflectance, which causes the bubbles to resonate under ultrasonic clinical frequency ranges of $1-10 \mathrm{MHz}$. Another example related to imaging is echo particle image velocimetry (echo-PIV), which is a non-invasive flow characterization method, where microbubbles act as flow tracers. ${ }^{95}$ This technique is based on backscattered ultrasound waves from bubbles, and provides accurate velocity measurements in cardiovascular flows. ${ }^{100,101}$

Apart from imaging, microbubbles are also used with ultrasound for therapeutic applications, including tissue/organ-specific drug and gene delivery. ${ }^{102-104}$ In these applications, the bubble surface (shell) may carry the molecule of interest. After bubbles are injected, ultrasonic waves at a specific frequency and amplitude impose pressure waves on the bubbles to release the drug molecules. ${ }^{105}$

In all the applications discussed previously, the size of the microbubbles is an important factor that can limit their functionality. ${ }^{9,106,107}$ However, commercially available microbubbles are often poly-disperse, ${ }^{10,108}$ which leads to heterogeneous responses under ultrasound. For this reason, several microfluidics-based techniques have been developed to generate mono-disperse microbubbles that have narrow size distributions. ${ }^{12,90,95,109-114}$ Techniques for microbubble generation in microfluidics use axisymmetric ${ }^{115}$ and symmetric ${ }^{95}$ flow-focusing channels, $\mathrm{T}$ junctions, ${ }^{111}$ and microneedles. ${ }^{116}$ While these microfluidics-based methods create bubbles that are mono-disperse, achieving sub $10-\mu \mathrm{m}$ diameter microbubbles, which are desirable in ultrasound imaging and therapy applications, ${ }^{117,118}$ is still challenging, and requires complex microfabrication techniques. $^{12,119}$

To overcome these challenges, our group recently reported a microfluidic bubble shrinkage technique that generates sub-10 $\mu \mathrm{m}$ diameter microbubbles and achieves easily tunable microbubble sizes between $1-10 \mu \mathrm{m}$. This technique is based on applying vacuum pressure in a polydimethylsiloxane (PDMS) based microfluidic chip to deplete air from the liquid-filled 
microchannels, so that suspended microbubbles shrink. ${ }^{90}$ The bubble-suspending microchannels are fabricated adjacent to vacuum microchannels, through which the negative pressure is applied.

In this chapter, we develop a theoretical framework that relates the size of the microbubbles produced and the microfluidic system's experimental input parameters, such as the lipid concentration in the liquid, and the gas-liquid surface tension. We systematically characterize the microbubble shrinkage performance of this technique via a series of experiments. We then develop and experimentally validate a mathematical model to predict and control the microbubble shrinkage process. Finally, by asymptotic analysis, we reduce the full mathematical model, which is only solvable numerically, to a simplified form that we solve analytically to produce an explicit relationship between the microbubble size and the experimental input parameters. We anticipate that this simple mathematical expression will be useful for engineering specific microbubble sizes in many biomedical applications, such as contrast-enhanced ultrasound imaging.

\subsection{Methods}

\subsubsection{Chemical Preparation}

Encapsulated microbubbles are generated in a microfluidic chip where air is used as the bubble gas core and a lipid solution as the encapsulation structure. We prepare two different lipid mixtures. In the first lipid mixture (M1), 1,2-distearoyl-sn-glycero-3-phosphoethanolamine-N((polyethylene glycol-5000)folate) (ammonium salt) (DSPE-PEG5F-15) (Avanti Polar Lipids) is mixed with 1,2-distearoyl-sn-glycero-3-phosphocholine (DSPC) at 9:1 ratio in saline (1.5 mg mL${ }^{1}$ ). Glycerol (Sigma Aldrich Corporation) and Pluronic F-68 (Fisher Scientific) are then added to the mixture in a 1:1:1 volumetric ratio to make the aqueous solution. The second lipid mixture (M2) is prepared similarly with the same molar/volumetric ratios, with the only difference that in the second mixture 1,2-distearoyl-sn-glycero-3-phosphoethanolamine-N-[Methoxy(Polyethylene glycol)-5000)folate) (ammonium salt) is used instead of DSPE-PEG5F-15. We measure the interfacial tension, $\sigma$, between the aqueous solution and air using the pendant-drop method. ${ }^{120}$ 


\subsubsection{Experimental Setup}

We pattern a cross flow-focusing microfluidic design (Figure 2.1) on a wafer substrate using a photolithography method. The height of all channels, including the vacuum channels, is $80 \mu \mathrm{m}$. We use the wafer as a mold for making PDMS microfluidic channels by soft lithography.

The injection of the aqueous solution is performed using a high-precision constant-flow-rate syringe pump (Harvard Instruments). A pressure gauge (Omega Engineering Inc.) is used to regulate the air pressure. The vacuum pressure is adjusted using a Mityvac hand vacuum pump (Mityvac). We conduct the experiments by injecting the aqueous solution at a constant flow rate

of $4 \mu \mathrm{Lmin} \min ^{-1}$ while air is supplied at a constant relative pressure of $27.6 \mathrm{kPa}$, and vacuum pressure is applied through the vacuum connections.

We image the bubbles at approximately the centre of each serpentine segment (red dashed rectangular area in Figure 2.1) using a high-speed camera (Phantom M110, Vision Research) attached to an inverted microscope (Olympus Corp.). The images are analysed by MATLAB (Mathworks) software using imfindcircles function to find the bubble size variation along the microfluidic channel.

The initial size of bubbles generated right after the flow-focusing junction is larger than the channel height, so bubbles are confined to discoid shapes. In order to accurately characterize bubble sizes, we use the bubbles' projected diameters to calculate the volume of the discoid bubbles, using a mathematical approach described elsewhere. ${ }^{90,121}$ 


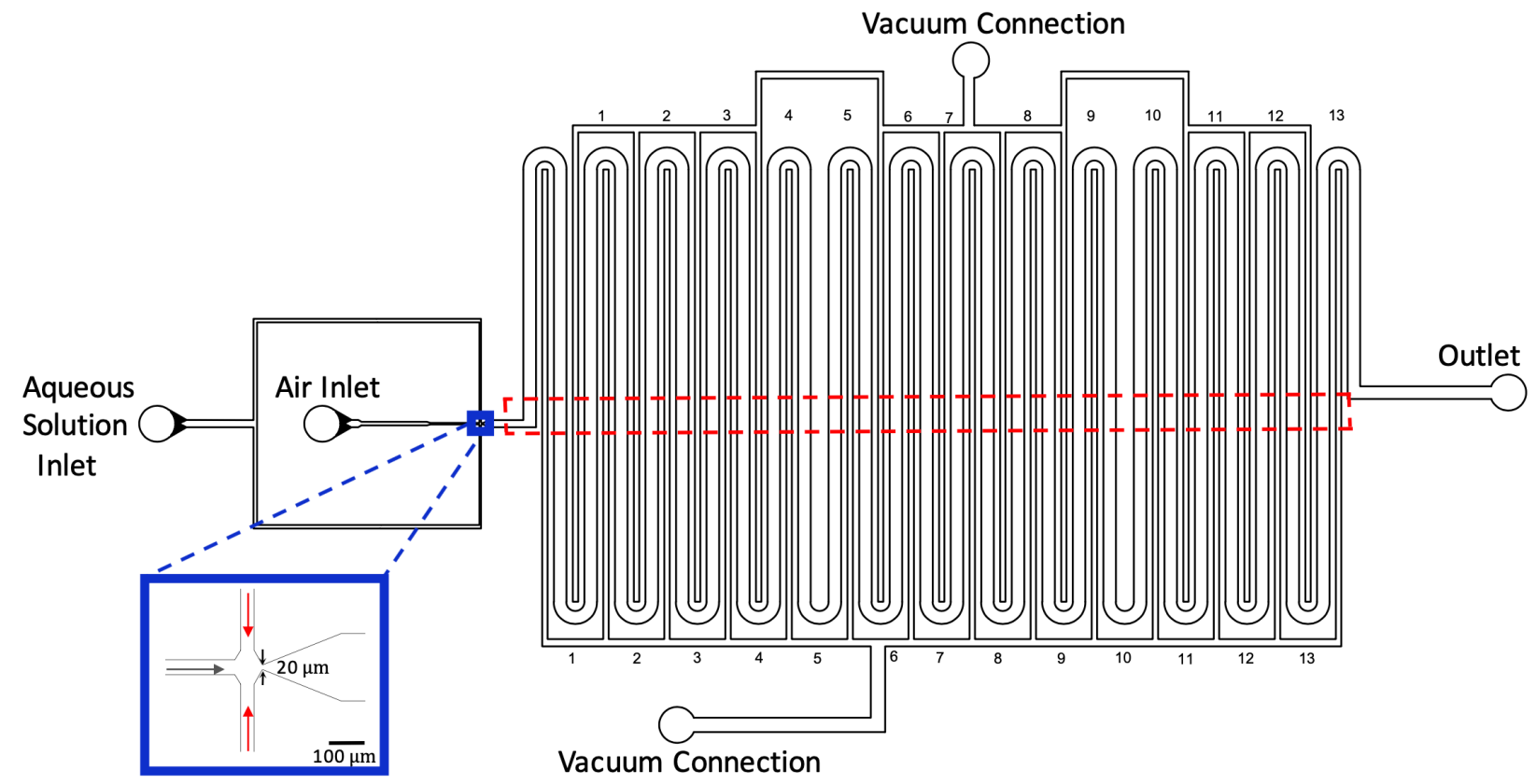

Figure 2.1 A schematic design of the microfluidic system containing a serpentine liquid-filled channel with a total length of $350 \mathrm{~mm}$. Microbubbles are generated at a $20 \mu \mathrm{m}$ width junction, where flows of air (central channel, grey arrow) and aqueous solution (side channels, red arrows) meet orthogonally. Vacuum is applied through two connections to achieve a uniform vacuum pressure across the entire chip. The bubble size variation is monitored by moving the microscope within the area depicted by red dashed lines. Images are captured when bubbles pass through this area.

\subsection{Results and Discussions}

\subsubsection{Experimental Results}

In order to study the effect of vacuum pressure on bubble shrinkage, we conduct experiments using a range of vacuum pressures $P_{v}=0$ to $-87 \mathrm{kPa}$, and with lipid mixture M1 (described in Experimental Methods). We also isolated the effect of other design parameters, such as gas core and channel dimensions, on bubble shrinkage by keeping them constant throughout our experiments. We note that the absolute pressure in the vacuum channel is equal to $P_{v}+P_{a t m}$, where $P_{a t m}$ is the atmospheric pressure. 
The size of bubbles generated at microfluidic junctions is governed by the orifice geometry, gas pressure, liquid flow rate, liquid viscosity, and interfacial tension. ${ }^{12,95,122}$ In this set of experiments, we maintain a constant initial microbubble radius $R=64 \pm 1 \mu \mathrm{m}$ (corresponding to a volume of $V=11 \times 10^{5} \mu \mathrm{m}^{3}$ ). This is the microbubble initial radius at the generation location, before exposure to vacuum shrinkage.

Figure 2.2 shows the plot of microbubble volume $V$ versus position in the microchannel $l$. At the baseline vacuum pressure $P_{v}=0$, the bubbles experience a $\sim 60 \%$ reduction in volume by the end of the microchannel (after travelling approximately $350 \mathrm{~mm}$ ). Increasing the vacuum pressures cause further reduction in the bubble size. A maximum final size reduction of $\sim 99 \%$ is achieved for vacuum pressures $P_{v} \leq-60 \mathrm{kPa}$. We note that for vacuum pressure $P_{v} \leq-60 \mathrm{kPa}$, the final bubble size is on the order of $\sim 1 \mu \mathrm{m}$, which approaches the diffraction limit of light, making the bubbles difficult to characterize.

We measure the aqueous solution air-liquid interfacial tension $\sigma=36.60 \mathrm{mN} \mathrm{m}^{-1}$. To systematically study the dependence of the final microbubble size on interfacial tension, we prepare aqueous solutions with two other interfacial tensions by diluting the original solution M1 in saline to achieve interfacial tensions $\sigma=48.29$ and $43.41 \mathrm{mN} \mathrm{m}^{-1}$.

Figure 2.3 shows the experimental results of bubble shrinkage using aqueous solutions with three different surface tensions. We find that the bubbles generated with solutions that have higher surface tensions are initially larger. When the interfacial tension $\sigma=36.60 \mathrm{mN} \mathrm{m}^{-1}$, bubbles are formed with an initial radius $R_{0}=61 \mu \mathrm{m}$ (volume $V=9.46 \times 10^{5} \mu \mathrm{m}^{3}$ ), and their radius decreases by $\sim 62 \%$ by the end of the channel, when exposed to a vacuum pressure $P_{v}=-50 \mathrm{kPa}$. Under the same vacuum pressure, $P_{v}=-50 \mathrm{kPa}$, microbubbles with initial radius $R_{0}=94 \mu \mathrm{m}$ (volume $V=3.50 \times 10^{6} \mu \mathrm{m}^{3}$ ), and interfacial tension $\sigma=48.29 \mathrm{mN} \mathrm{m}^{-1}$, shrink by $\sim 43 \%$.

When we apply a vacuum pressure $P_{v}=-87 \mathrm{kPa}$, bubbles generated using solutions with interfacial tensions $\sigma=36.60 \mathrm{mN} \mathrm{m}^{-1}$ and $43.41 \mathrm{mN} \mathrm{m}^{-1}$ shrink to an approximate radius $R=$ $0.5 \mu \mathrm{m}$ by the channel positions $l=250 \mathrm{~mm}$ and $325 \mathrm{~mm}$, respectively. No data is collected 
beyond that point. This shows $\sim 99 \%$ reduction in radius when interfacial tension $\sigma=$ $36.60 \mathrm{mN} \mathrm{m}^{-1}$ and $\sim 68 \%$ reduction when interfacial tension $\sigma=48.29 \mathrm{mN} \mathrm{m}^{-1}$.

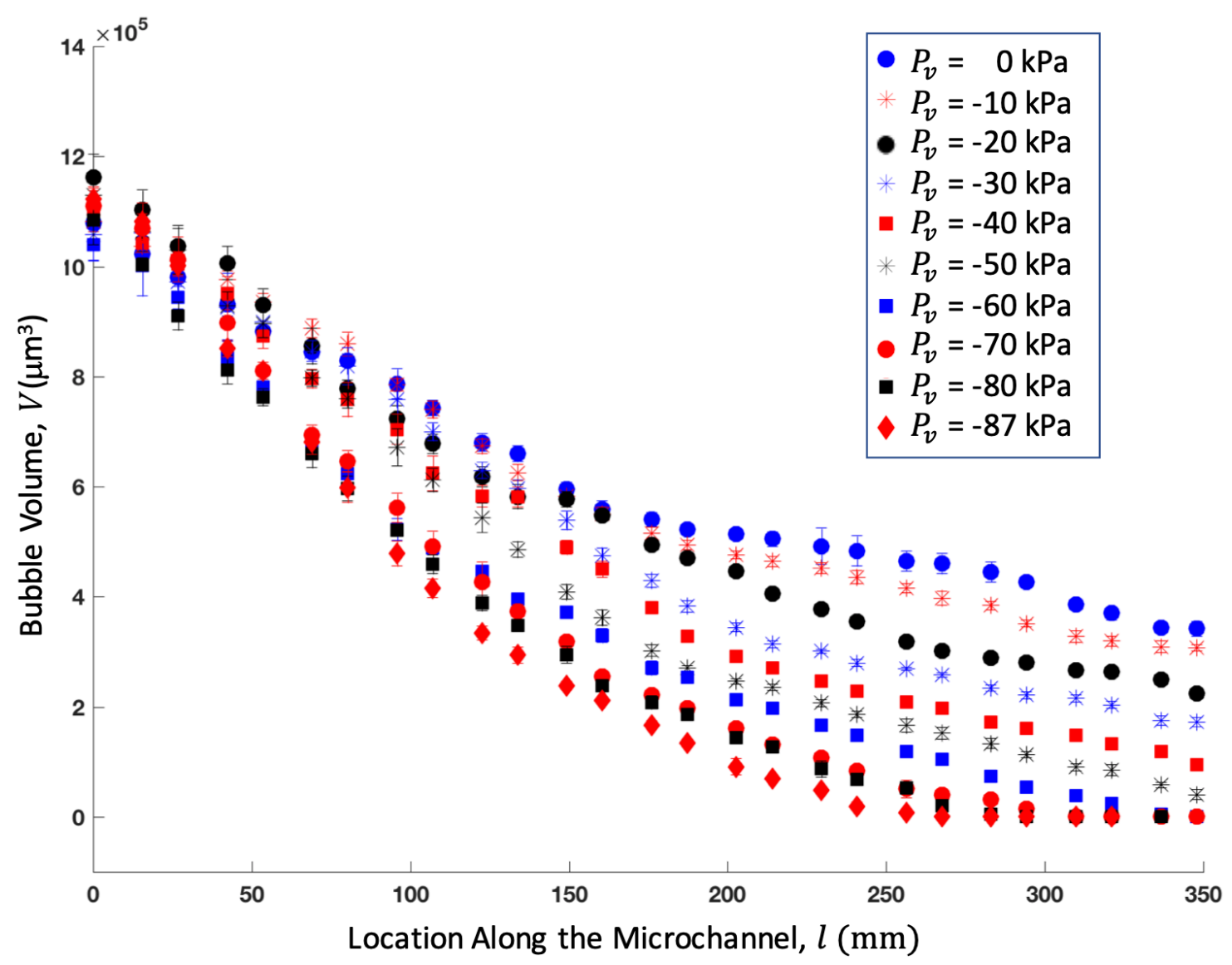

Figure 2.2 A plot of the experimental results for the bubble volume $V$ versus the location $l$, along the microchannel at ten different vacuum pressures. The error bars indicate the standard deviations calculated by analysing $>10$ bubbles for each data point. In all experiments, the mixture surface tension, aqueous liquid flow rate, and air pressure are kept constant at $\sigma=36.60 \mathrm{mN} \mathrm{m}^{-1}$, $4 \mu \mathrm{L} \mathrm{min}^{-1}$, and $27.6 \mathrm{kPa}$, respectively. The plot shows a monotonic trend of increasing microbubble shrinkage rate with increasing vacuum pressure. 

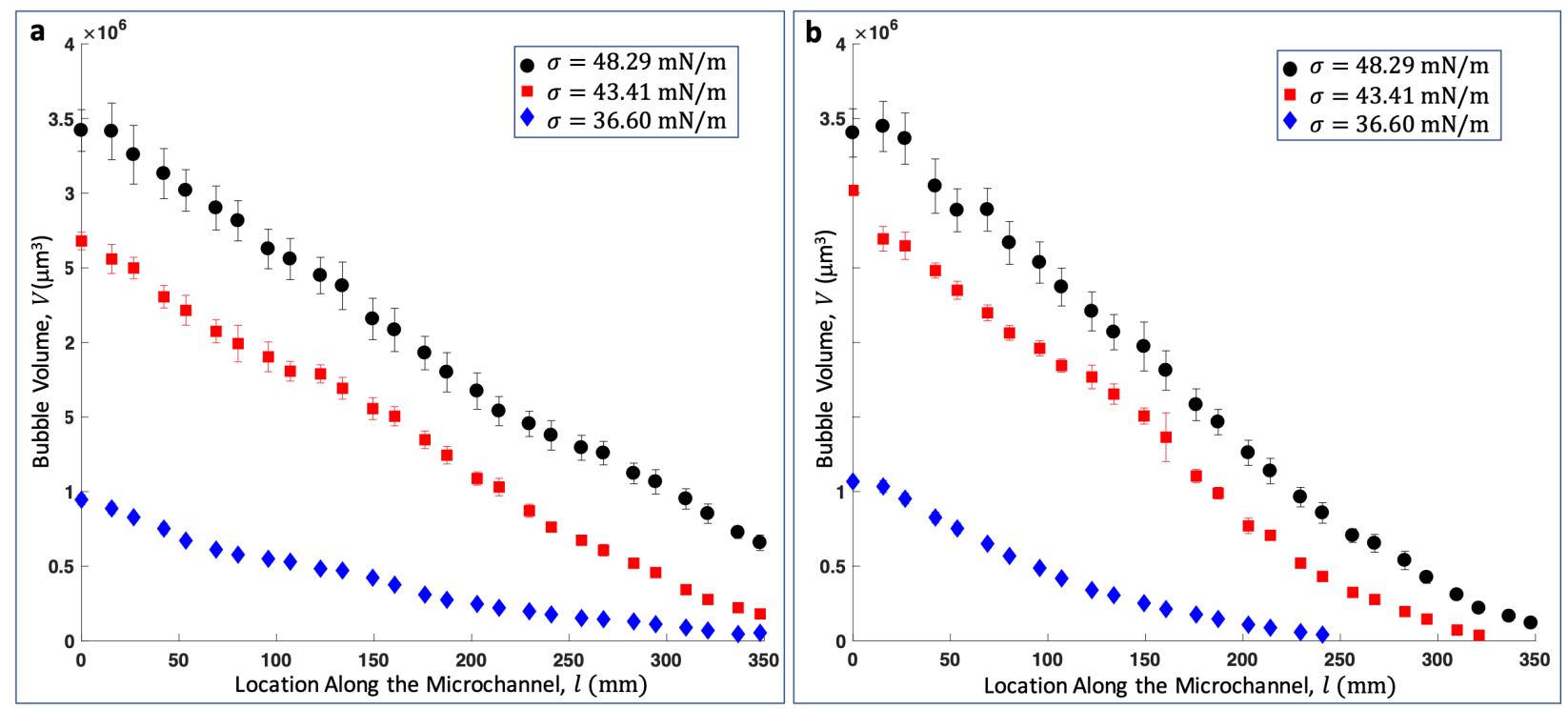

Figure 2.3 Experimental results for the bubble volume $V$ versus the location along the microchannel $l$ for aqueous solutions with three different surface tensions. The error bars show standard deviations calculated by analysing $>10$ bubbles for each data point. Here, the vacuum pressure is held constant at (a) $P_{v}=-50 \mathrm{kPa}$, and (b) $P_{v}=-87 \mathrm{kPa}$. In all experiments, the aqueous liquid flow rate and air pressure are kept constant at $4 \mu 1 \mathrm{~min}^{-1}$ and $27.6 \mathrm{kPa}$, respectively. Bubbles generated at higher surface tensions are initially larger and shrink more throughout the channel.

We also observe that changing the lipid mixture affects the shrinkage behaviour. Bubbles generated using mixture M2 demonstrate less shrinkage compared to bubbles formed from mixture M1. For example, the radius $R$ of bubbles generated using mixture M2 decreases by $\sim 52 \%$ by the end of the microchannel, while we observe a $\sim 99 \%$ reduction in radius $R$ for bubbles formed from mixture M1 using the same vacuum pressure $P_{v}=-87 \mathrm{kPa}$. Here, the surface tensions $\sigma$ of the two mixtures are similar, i.e., $\sigma=36.60 \mathrm{mN} \mathrm{m}^{-1}$ for mixture $\mathrm{M} 1$ and $\sigma=37.66 \mathrm{mN} \mathrm{m}^{-1}$ for mixture M2.

\subsubsection{Mathematical Modelling}

When microbubbles are generated in the aqueous solution, lipid molecules, which consist of a hydrophilic head and a hydrophobic tail, migrate towards and deposit onto the interface of the liquid/gas covering the bubbles, forming a self-assembled layer called a shell. ${ }^{109}$ The main function of shells in microbubble technology is to reduce the surface tension and extend the life-time of 
microbubbles from seconds to years. ${ }^{123}$ Microbubble stability is studied extensively in the literature because the lifetime and shell property of microbubbles are important in their application. ${ }^{109,124-131}$ Here, we apply some of the physical concepts from microbubble stability to model the shrinkage behaviour of the microbubbles in our microfluidic device, under vacuum pressure.

We apply a stability analysis of a typical microbubble with known shell encapsulation, and with a gas core of known transport properties. We neglect the effects of flow on the shrinkage once the bubble is generated, assuming that the bubble is continuously advected downstream with the flow. Therefore, the modeling is simplified to a microbubble stability analysis over time. This simplification assists in correlating the experimental data taken at each section of the serpentine channel to the time passed for the bubbles to reach to that section after they are generated at the flow-focusing junction.

Microbubble lifetimes were first mathematically modelled by Epstein and Plesset, where they considered a "clean" bubble, without encapsulation, in the bulk of a liquid and under diffusion-limited physics. ${ }^{132}$ Without encapsulation, a "clean" microbubble is unstable, dissolving in less than a second. Microbubbles stabilized by encapsulations can be modelled the same way, but with modified shell properties. ${ }^{125,131,133}$ Here, we follow the same approach to model our shrinking bubbles.

Assuming an encapsulated microbubble is fully immersed in a liquid, and the diffusion timescale is much less than the dissolution time, the steady state conservation equation of gas core molecules in spherical coordinate is reduced to,

$$
\frac{1}{r^{2}} \frac{\mathrm{d}}{\mathrm{d} r}\left(r^{2} \frac{\mathrm{d} C}{\mathrm{~d} r}\right)=0
$$

where $C$, and $r$ are concentration (mole/volume) of the gas molecules in the aqueous bulk, and radial coordinate, respectively. 
As shown in Figure 2.4, we can assume the following conditions for a single bubble with a radius $R$ and a shell thickness $\delta$, immersed in an aqueous solution,

$$
\begin{gathered}
C(r<(R-\delta))=C_{i n}, \\
C(r=(R-\delta))=C_{w}, \\
C(r=R)=C_{R}, \\
C(r \rightarrow \infty)=C_{f},
\end{gathered}
$$

where $C_{i n}, C_{w}, C_{R}$, and $C_{f}$ are the gas concentration inside the bubble, at the inner bubble wall (shell), at the bubble outer shell, and in the liquid bulk (aqueous solution) far from the bubble, respectively. Due to the laminar flow in the microfluidic channel, diffusion is the main transport mechanism responsible for gas release from bubbles to the vacuum channels, and thus the advection terms are neglected. Also, we neglect the effect of channel entrance and exit, and any bubble-bubble interactions on the gas transport mechanism.

Conservation of mass through the shell provides one boundary condition for Eq. (2.1),

$$
-\left.k_{g} \frac{\mathrm{d} C}{\mathrm{~d} r}\right|_{r=R}=h_{g}\left(C_{w}-C_{R}\right)
$$

where $k_{g}$ and $h_{g}$ are the gas diffusion coefficient in the aqueous phase, and shell permeability coefficient, respectively.

Solving Eq. (2.1) with the boundary conditions $C(r \rightarrow \infty)=C_{f}$ and Eq. (2.3) gives, 


$$
C(r)=R^{2}\left(\frac{C_{w}-C_{f}}{r\left(\frac{k_{g}}{h_{g}}+R\right)}\right)+C_{f}
$$

Therefore,

$$
C_{R}=\frac{\frac{h_{g}}{k_{g}} R C_{w}+C_{f}}{1+R \frac{h_{g}}{k_{g}}} .
$$




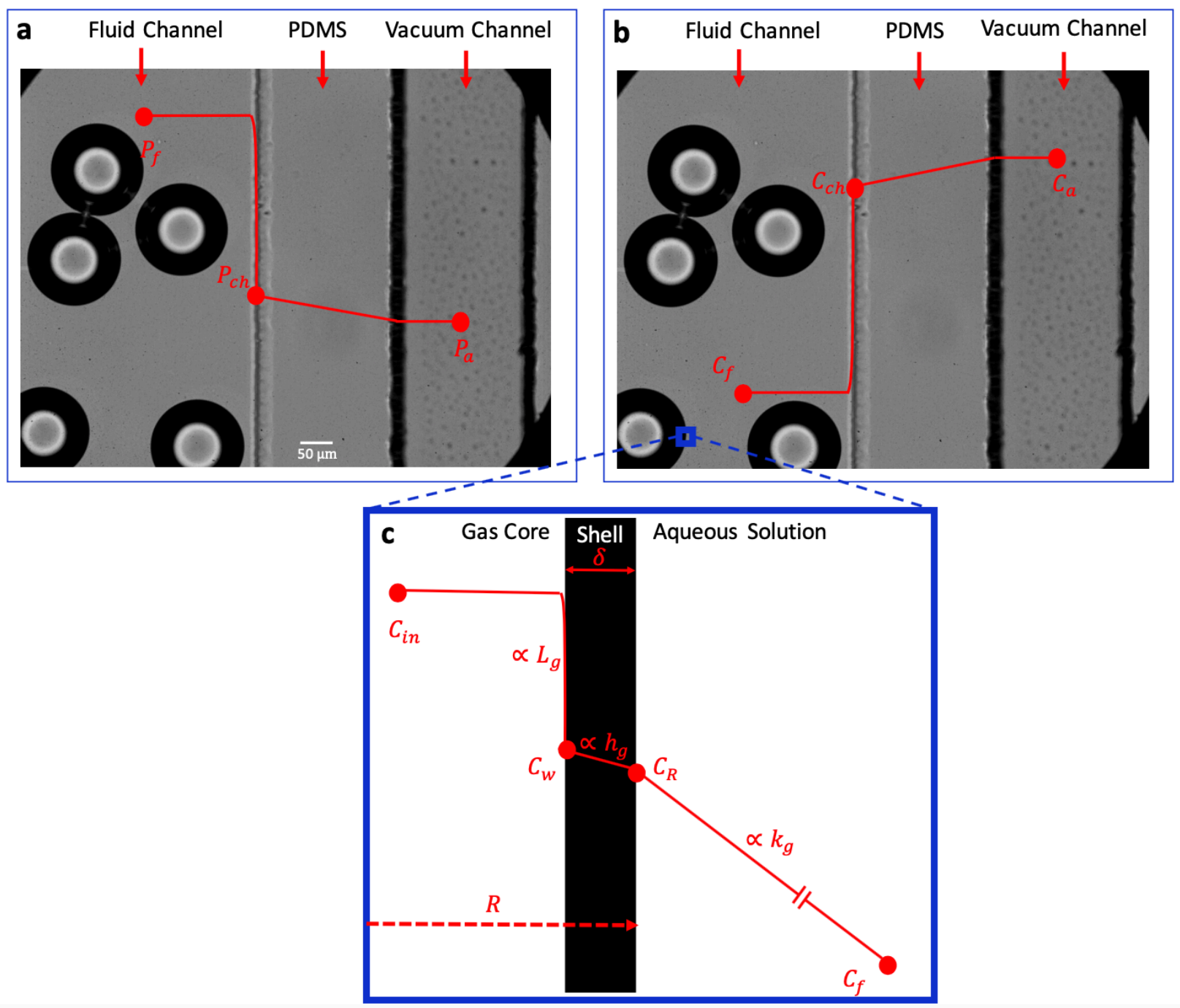

Figure 2.4 Microscopy images showing the fluid channel, PDMS bulk, and the vacuum channel. (a) The schematic profile overlaid on the images, of absolute pressure distribution and (b) the concentration distribution across the microfluidic channel. The changes of absolute pressure and air concentration through the PDMS bulk is negligible. Ostwald's law governs the relationship between the absolute pressure and air concentration in the fluid channel, and pressure and air concentration in the bulk PDMS. (c) A schematic diagram of the concentration changes across a microbubble shell.

Applying Ostwald's law and the ideal gas law, we obtain the following relation between the gas core pressure and inner shell concentration (Figure 2.4c), 


$$
\begin{gathered}
C_{w}=L_{g} C_{i n} \\
C_{\text {in }}=\frac{P_{\text {in }}}{R_{G} T},
\end{gathered}
$$

where $L_{g}, P_{i n}, R_{G}$, and $T$ are the Ostwald coefficient of the gas core, gas pressure inside the bubble, universal gas constant, and gas temperature, respectively.

Similarly, as shown in Figure 2.4b, the concentration of gas in the aqueous solution far from the bubble, $C_{f}$, is related to the concentration at the fluid channel wall outside of the aqueous phase, $C_{c h}$,

$$
C_{f}=f L_{g} C_{c h}
$$

where, $f$ is typically defined as the level of saturation of the aqueous solution. However, since the level of saturation is not homogenous throughout the liquid in our system, we refer to $f$ as an effective level of saturation.

The nominal level of saturation varies along the aqueous solution channel. For example, the value near the inlet may be different from that close to outlet. This inhomogeneity may arise from the effect of the pressure drop inside the aqueous solution, ${ }^{134,135}$ and diffusion through the PDMS bulk. ${ }^{6}$ Therefore, for simplicity and convenience, we define $f$ as an effective level of saturation, which we use as a fitting parameter in our model, to account for all of the inhomogeneities in liquid saturation.

It has been shown in the literature that for high gas or air permeability (diffusivity, porosity, and solubility) ${ }^{136}$ and thin PDMS membranes (175 $\mu \mathrm{m}$ wall thickness in our case), equilibrium across the thicknesses can be achieved very quickly, often within a few seconds. ${ }^{137}$ Neglecting the 
concentration difference across the bulk PDMS, i.e., assuming $C_{c h} \approx C_{a}$, and assuming the gas inside the vacuum channel is an ideal gas, we can write,

$$
C_{f}=f L_{g} \frac{P_{a}}{R_{G} T^{\prime}}
$$

where $P_{a}$ is the pressure inside the vacuum channel and is determined by the vacuum pressure, $P_{v}$,

$$
P_{a}=P_{a t m}+P_{v}
$$

To relate the bubble size to the gas concentration, we apply the mass conservation equation for the gas core assuming a bubble volume of $V=4 / 3 \pi R^{3}$ and surface area of $A=4 \pi R^{2}$,

$$
\frac{\mathrm{d}}{\mathrm{d} t}\left(\frac{4}{3} \pi R^{3} C_{\text {in }}\right)=-\left(4 \pi R^{2}\right) h_{g}\left(C_{w}-C_{R}\right)
$$

Due to interfacial tension effects on the bubble shell, Laplace's equation governs the pressure difference across the shell. We note that, for an encapsulated microbubble, the surface tension, $\sigma$, is a function of bubble radius, $R$, and therefore, a dilatational surface elasticity $E_{S}$ can be defined as the derivative of surface tension with respect to fractional change in the interfacial area, ${ }^{125,130,131,138}$ 


$$
\begin{gathered}
P_{\text {in }}-P_{f}=\frac{2 \sigma(R)}{R}, \\
\sigma(R)=\sigma_{0}+E_{S}\left(\left(\frac{R}{R_{0}}\right)^{2}-1\right),
\end{gathered}
$$

where $P_{\text {in }}, R_{0}$, and $\sigma_{0}$ are the pressure inside bubble, initial radius of the stress-free bubble conformation, and initial stress-free interfacial tension of the bubble, respectively.

Now, we can substitute Eq. (2.11) into Eq. (2.6), assuming $P_{f}$ is equal to the ambient pressure, $P_{a t m}$,

$$
\begin{gathered}
C_{w}=L_{g} \frac{P_{a t m}+\frac{2 \sigma_{0}}{R}+\frac{2 E_{s}}{R}\left(\left(\frac{R}{R_{0}}\right)^{2}-1\right)}{R_{G} T}, \\
C_{\text {in }}=\frac{P_{a t m}+\frac{2 \sigma_{0}}{R}+\frac{2 E_{s}}{R}\left(\left(\frac{R}{R_{0}}\right)^{2}-1\right)}{R_{G} T} .
\end{gathered}
$$

Similarly, we can substitute Eqs. (2.8) and (2.12) into Eq. (2.5),

$$
C_{R}=\frac{L_{g}}{R_{G} T\left(1+R \frac{h_{g}}{k_{g}}\right)}\left(\frac{h_{g}}{k_{g}} R\left(P_{a t m}+\frac{2 \sigma_{0}}{R}+\frac{2 E_{s}}{R}\left(\left(\frac{R}{R_{0}}\right)^{2}-1\right)\right)+f P_{a}\right) .
$$

Finally, substituting Eqs. (2.12) and (2.13) into (2.10), we obtain the governing equation for the time-dependent bubble radius as follows, 


$$
\frac{\mathrm{d} R}{\mathrm{~d} t}=-\frac{3 k_{g} L_{g}}{\frac{k_{g}}{h_{g}}+R}\left(\frac{(1-f) P_{a t m}-f P_{v}+\frac{2 \sigma_{0}}{R}+\frac{2 E_{s} R}{R_{0}^{2}}-\frac{2 E_{s}}{R}}{3 P_{a t m}+\frac{4 \sigma_{0}}{R}+\frac{8 E_{s} R}{R_{0}^{2}}-\frac{4 E_{s}}{R}}\right) .
$$

Eq. (2.14) can be nondimensionalized by defining the following dimensionless parameters,

$$
\begin{array}{cc}
\hat{t}=\frac{3 t k_{g} L_{g}}{R_{0}{ }^{2}}, & \hat{R}=\frac{R}{R_{0}}, \\
\widehat{k_{g}}=\frac{k_{g}}{h_{g} R_{0}}, & \hat{\sigma}=\frac{2 \sigma_{0}}{P_{a t m} R_{0}}, \\
\hat{E}=\frac{2 E_{s}}{P_{a t m} R_{0}}, & \widehat{P_{v}}=\frac{P_{v}}{P_{a t m}},
\end{array}
$$

where $\hat{t}, \hat{R}$, and $\widehat{P}_{v}$ are dimensionless values representing timescale, bubble radius, and vacuum pressure, respectively. $\widehat{k_{g}}, \hat{\sigma}$, and $\widehat{E}$ are dimensionless values representing diffusion coefficient, interfacial tension, and elasticity, respectively.

This allows us to write Eq. (2.14) as,

$$
\frac{\mathrm{d} \hat{R}}{\mathrm{~d} \hat{t}}=-\frac{1}{\widehat{k_{g}}+\hat{R}}\left(\frac{(1-f)-f \widehat{P}_{v}+\frac{\hat{\sigma}}{\hat{R}}+\hat{E} \hat{R}-\frac{\hat{E}}{\hat{R}}}{3+\frac{2 \hat{\sigma}}{\hat{R}}+4 \hat{E} \hat{R}-\frac{2 \hat{E}}{\hat{R}}}\right) .
$$

In addition to modeling bubble size changes over time, we also use COMSOL Multiphysics software to conduct a 2D numerical simulation of typical gas (air) convection through a long (350 
$\mathrm{mm}$ ) microfluidic channel filled with fully saturated liquid (water) at ambient pressure. Here, we apply three different concentration conditions imposed by Ostwald's law on the PDMS wall for each vacuum pressure (i.e., $P_{v}=0,-50$, and $-87 \mathrm{kPa}$ ). Figure 2.5 shows the numerical solution of concentration variation along the centreline of a $350 \mathrm{~mm}$ long liquid filled microfluidic channel and the 2D geometry used in the simulation. The simulations show that, regardless of the vacuum pressure magnitude, the PDMS wall thickness is small enough that the air concentration in the aqueous flow attains its equilibrium value in less than $\sim 15 \%$ of the channel length. This effect is thus ignored in our modeling and can be made smaller if the PDMS wall between the liquid filled microfluidic channel and the vacuum channel is thinner.

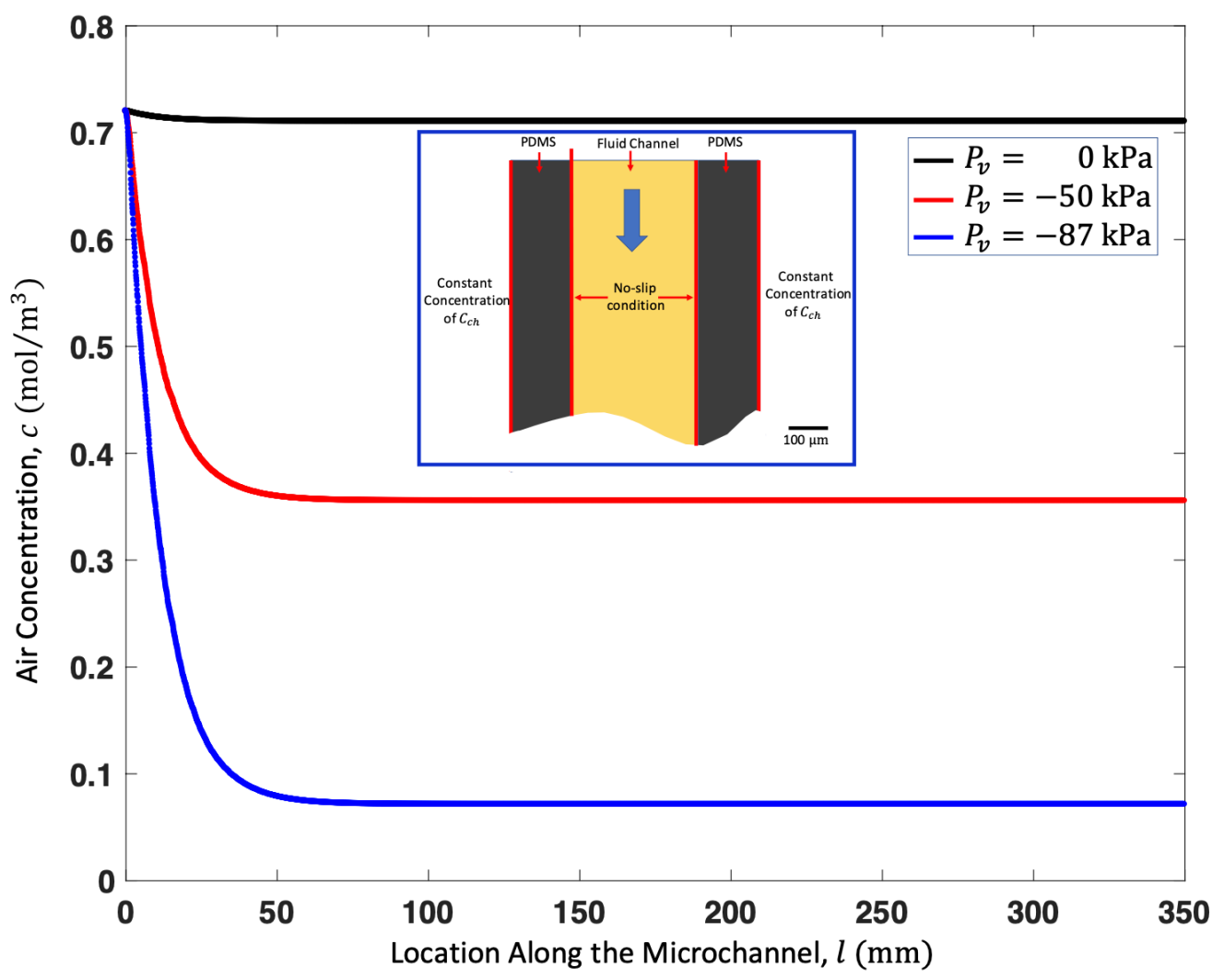

Figure 2.5 Simulation results representing the concentration of dissolved air along the channel centreline at three different vacuum pressures, $P_{v}=0,-50$ and $-87 \mathrm{kPa}$. In simulations, we assume that the microfluidic channel is filled with water only, and thus the effect of bubble flow is neglected. The effect of channel entrance on the air concentration within the liquid is diminished once the liquid travels $50 \mathrm{~mm}$ along the channel length. The inset shows the 2D geometry used in the simulation and the boundary conditions applied. 
We can solve Eq. (2.16) by separation of variables, subject to the initial condition $\hat{R}=1$ to give,

$$
\hat{t}(\hat{R})=\int_{1}^{\hat{R}}-\left(\widehat{k_{g}}+\tilde{R}\right)\left(\frac{3+\frac{2 \hat{\sigma}}{\tilde{R}}+4 \hat{E} \tilde{R}-\frac{2 \widehat{E}}{\tilde{R}}}{(1-f)-f \widehat{P_{v}}+\frac{\hat{\sigma}}{\tilde{R}}+\hat{E} \tilde{R}-\frac{\hat{E}}{\tilde{R}}}\right) d \tilde{R} .
$$

The integral on the right-hand side of Eq. (2.17) can be evaluated analytically to give,

$$
\hat{t}(\hat{R})=g(\widehat{R})-g(1)
$$

where 


$$
\begin{aligned}
& g(\hat{R})=\left[4 \widehat{E}^{3} \widehat{k_{g}}+(-1+f+\right. \\
& \left.f \widehat{P}_{v}\right)^{2}\left(-1+4 f\left(1+\widehat{P}_{v}\right)\right)+\widehat{E}\left(\widehat{k_{g}}(1-\right. \\
& \left.5 f\left(1+\widehat{P_{v}}\right)+4 f^{2}\left(1+\widehat{P_{v}}\right)^{2}\right)-2(-2+ \\
& \left.\left.5 f\left(1+\widehat{P}_{v}\right)\right) \hat{\sigma}\right)+2 \widehat{E}^{2}\left(5 f\left(1+\widehat{P}_{v}\right)-\right. \\
& \left.\left.2\left(1+\widehat{k_{g}} \hat{\sigma}\right)\right)\right] \times \\
& \frac{\operatorname{Arctan}\left(\frac{1-f\left(1+\widehat{P}_{v}\right)+2 \hat{E} \hat{R}}{\sqrt{-4 \hat{E}^{2}-\left(-1+f+f \widehat{P_{v}}\right)^{2}+4 \hat{E} \hat{\sigma}}}\right)}{\left[\hat{E}^{2} \sqrt{-4 \hat{E}^{2}-\left(-1+f+f \widehat{P}_{v}\right)^{2}+4 \hat{E} \hat{\sigma}}\right]}- \\
& {\left[\left(1+2 E^{\wedge} 2-5 f\left(1+\left(P_{-} v\right)^{\wedge}\right)+4 f^{\wedge} 2\left(1+\left(P_{-} v\right)^{\wedge}\right)^{\wedge} 2+E^{\wedge}\left(\left(k_{-} g\right)^{\wedge}(-1\right.\right.\right.} \\
& \left.\left.\left.+4 f\left(1+\left(P_{-} v\right)^{\wedge}\right)\right)-2 \sigma^{\wedge}\right)\right) \log \left(-\left(-1+f+f\left(P_{-} v\right)^{\wedge}\right) R^{\wedge}\right. \\
& \left.\left.+E^{\wedge}\left(-1+R^{\wedge} 2\right)+\sigma^{\wedge}\right)\right] /\left[2 E^{\wedge} 2\right]
\end{aligned}
$$

Equation (2.18) gives the dimensionless time at which a desired encapsulated microbubble radius is attained for a given vacuum pressure, an aqueous solution of known surface tension, gas diffusion coefficient, and effective level of gas saturation, and a bubble shell of known permeability and elasticity. We take vacuum pressure $P_{v}=0$ as the control experiment to obtain values for our fitting parameters, which are shell properties $\left(h_{g}, E_{s}\right)$ and the effective level of saturation $f$. We find a good agreement with the experimental data in predicting the bubble size versus time when $f=0.62, h_{g}=2.6 \times 10^{-5} \mathrm{~ms}^{-1}$, and $E_{s}=9 \times 10^{-3} \mathrm{Nm}^{-1}$. These values are all within the range of commonly used values in literature. ${ }^{125,131,133}$ We note that we maintain the same fitting parameter values for all experiments, including for solutions with different surface tensions and experiments under various vacuum pressures (Figure 2.6). We also keep the following parameters constant: $L_{g}=1.7 \times 10^{-2}$ and $k_{g}=2.05 \times 10^{-9} \mathrm{~m}^{2} \mathrm{~s}^{-1} .{ }^{125}$ 
The phenomenon of bubble shrinkage in the absence of vacuum pressure is reported in earlier publications when the saturation $f<1,{ }^{125,139}$ and observed here in our experiments. Our hypothesis for this somewhat unintuitive observation is the following.

One part of our fabrication process leaves the PDMS bulk inside a vacuum chamber for a few minutes to perform plasma-treatment on the channel surfaces. In order to maintain constant surface chemistry in all of the microfluidic devices we use, all of our experiments are conducted within 30 min of this treatment step. Therefore, the PDMS bulk is degassed at the time of each experiment, causing the PDMS bulk to absorb air from all directions as well as from the aqueous solution at a very low rate, yielding the effective level of saturation $f<1$.

To test this hypothesis, we perform a similar experiment with an air-saturated PDMS device at vacuum pressure $P_{v}=0$. As illustrated in Figure A.1, the shrinkage of the bubbles in the airsaturated PDMS device, when vacuum pressure $P_{v}=0$, is almost negligible, suggesting that the usage of degassed PDMS device in our experiments is the main contributor to our observed bubble shrinkage at vacuum pressure $P_{v}=0$.

When $\left|P_{v}\right|>0$, the highest flux of gas mass transfer occurs in the direction from the aqueous solution to the vacuum channel, which has the lowest resistance to mass transfer, causing the bubble to shrink at a higher rate.

In general, depending on the shell properties and effective level of saturation, the bubbles will either reach a stable final radius, or dissolve entirely. Our results show that, under the abovementioned conditions, bubble shrinkage continues if the vacuum pressure is applied. One can adjust the channel length in a way that, once the bubbles reach to the desired final size, they can be collected. Alternatively, the vacuum pressure can be adjusted accordingly so that the bubbles reach the channel outlet at the desired size. 

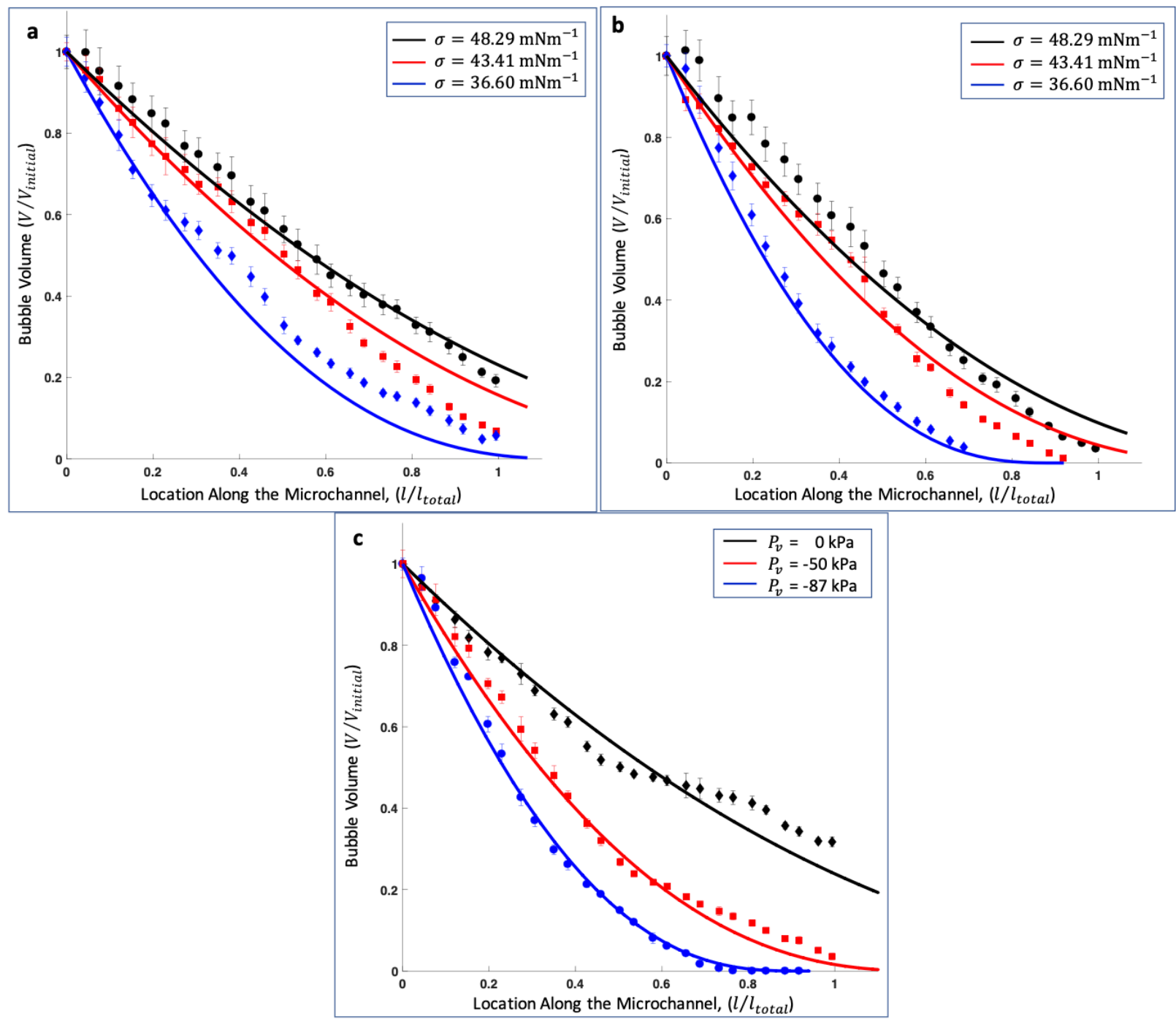

Figure 2.6 Dimensionless experimental and modeling results for the bubble volume $V / V_{\text {initial }}$ versus the location along the channel $l / l_{\text {total }}$. The results are shown for different surface tensions $\sigma$, while vacuum pressures (a) $P_{v}=-50 \mathrm{kPa}$, and (b) $P_{v}=-87 \mathrm{kPa}$. (c) Three different vacuum pressures with constant surface tension $\sigma=36.60 \mathrm{~m} \mathrm{Nm}^{-1}$. Data points state experimental results, while the solid lines represent the numerical solution of the mathematical model. The modeling results demonstrate a very good agreement with the experimental data. Here, initial bubble volume $V_{\text {initial }}=4 / 3 \pi R_{0}{ }^{3}$ and the microchannel length $l_{\text {total }}=350 \mathrm{~mm}$. In (a) and (b), initial bubble radius $R_{0}=89 \mu \mathrm{m}, 83 \mu \mathrm{m}$, and $61 \mu \mathrm{m}$, correspond to surface tensions $\sigma=$ $48.29 \mathrm{~m} \mathrm{Nm}^{-1}, 43.41 \mathrm{~m} \mathrm{Nm}^{-1}$, and $36.60 \mathrm{~m} \mathrm{Nm}^{-1}$, respectively. In (c), the initial bubble radius $R_{0}=63 \mu \mathrm{m}$. 
In all the experiments, the dimensionless surface tension $\hat{\sigma}$ and elasticity $\hat{E}$ are small quantities. Typically, $\hat{\sigma} \simeq O\left(10^{-2}\right)$ and $\left.\hat{E} \simeq O\left(10^{-3}\right)\right)$. In this limit, Eq. (2.18) is reducible to the simple expression,

$$
\hat{R}(\hat{t})=-\widehat{k_{g}}+\sqrt{\left(1+\widehat{k_{g}}\right)^{2}+\frac{2}{3}\left(\left(1+\widehat{P_{v}}\right) f-1\right) \hat{t}}
$$

which is in excellent agreement with the full analytical solution (Figure 2.7).

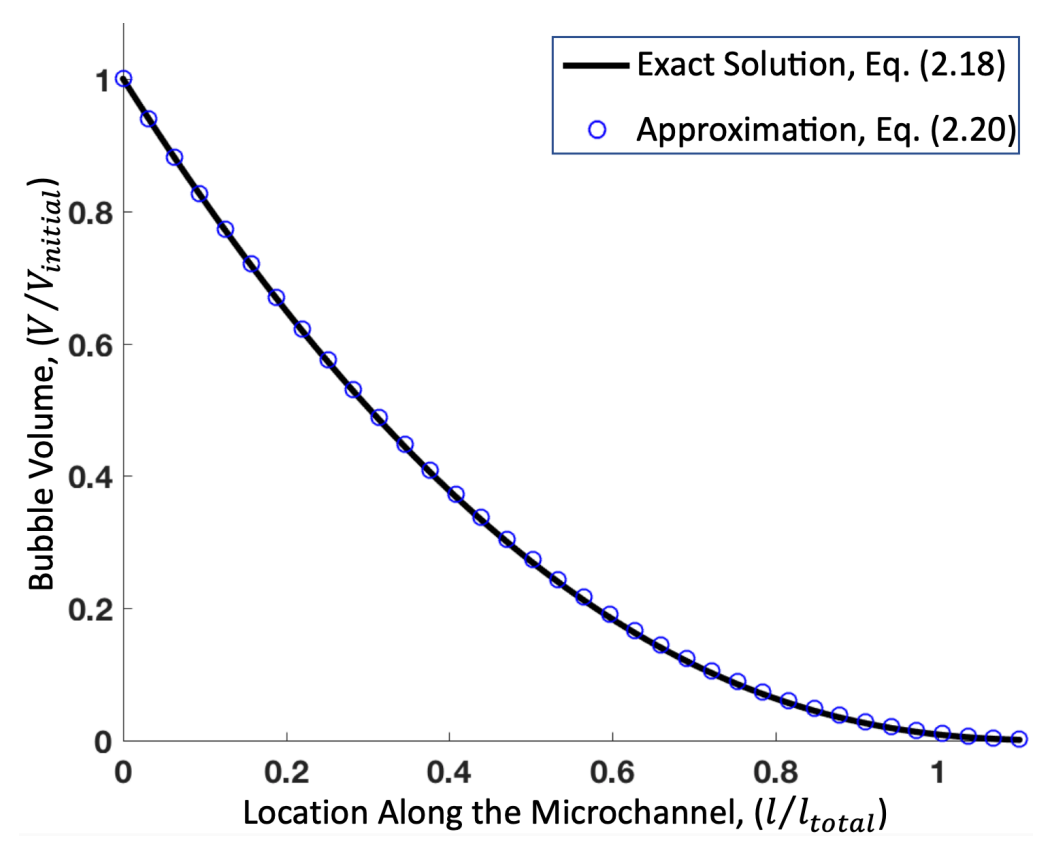

Figure 2.7 A plot of the dimensionless bubble volume $V / V_{\text {initial }}$ versus the position along the channel $l / l_{\text {total }}$. Here, the dimensionless parameters are $\hat{\sigma}=1.18 \times 10^{-2}, \widehat{P}_{v}=-0.5, \hat{E}=$ $2.90 \times 10^{-3}, \widehat{k_{g}}=1.27$, and $f=0.62$. The approximated solution (Eq. (2.20)) is almost identical to the exact solution (Eq. (2.18)).

Thus, this simplified expression of the bubble radius (Eq. (2.20)) is valid for all liquid solutions that have relatively small surface tension $\hat{\sigma}$ and dilatational surface elasticity $\hat{E}$. In these circumstances, our analysis shows that for a given initial bubble radius, the effect of liquid surface tension and dilatational surface elasticity of the bubble on the shrinkage rate is negligible. 
The agreement indicates that our shrinkage model is versatile enough to be applied to microfluidic systems where bubbly flows are mixed with flows with different surface tensions, such as biofluids. However, as shown in Figure 2.6a and Figure 2.6b, we still observe different shrinkage rates upon changing the liquid surface tension $\sigma$ since changes in the surface tension manifest themselves through changes in the initial bubble radius $R_{0}$.

Eqs (2.18), (2.19) and (2.20) together provide a clear and powerful method for determining the operating conditions required to generate any desired bubble size. Eqs. (2.18) and (2.19) explicitly encapsulate all of the physical variables within the system, and the way in which they influence the bubble evolution. As a result, even if experimental limitations provide bounds on what can be physically achieved for one particular parameter, this equation indicates how the other physical parameters can be manipulated to produce a desired bubble size.

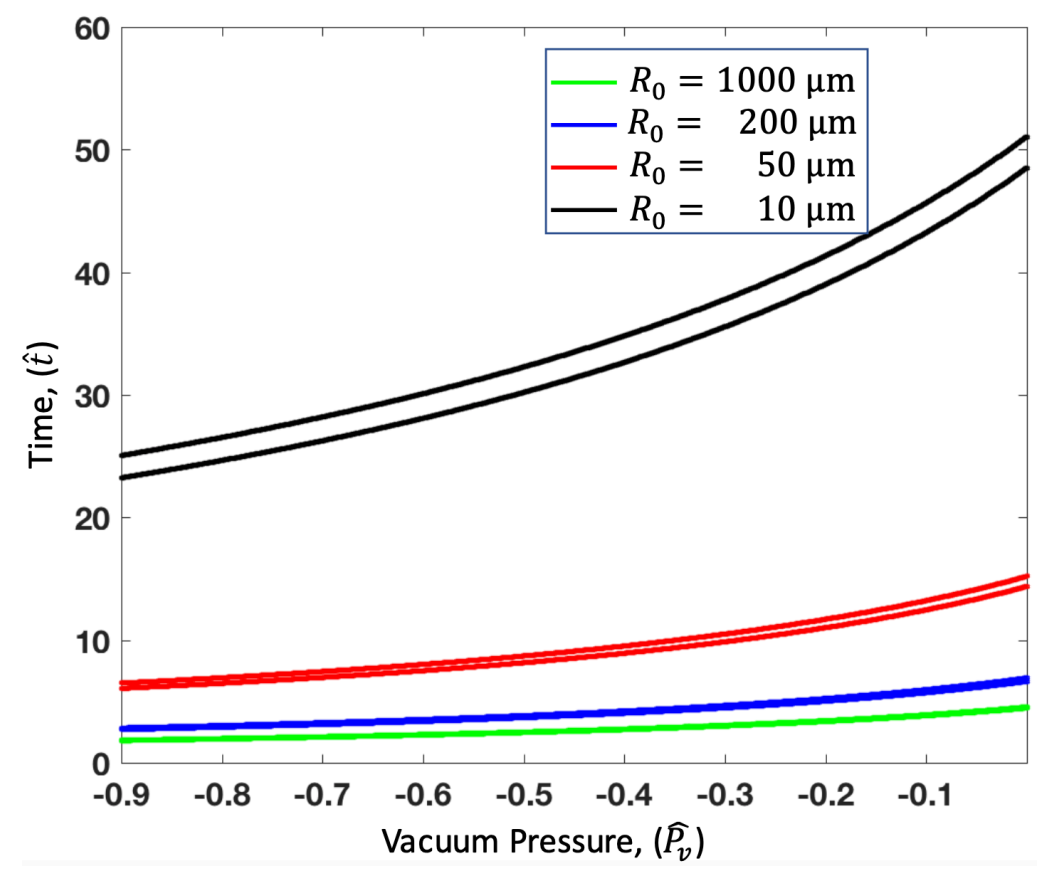

Figure 2.8 The dependence of shrinkage time required for bubbles to reach a radius $\widehat{R}=0.01$ (upper curve) and $\hat{R}=0.1$ (lower curve) given an initial radius, $R_{0}$, on vacuum pressure. The vacuum pressure $\widehat{P}_{v}$ is more effective on initially smaller bubbles.

The further reduced form of Eq. (2.20) takes the analytical expression a step further by providing an explicit form for the bubble radius as a function of the key parameters in this 
particular microfluidic system, namely the diffusion coefficient, effective level of saturation, shell permittivity, Ostwald's coefficient, and vacuum pressure. The result provides a mechanism for rapid prediction of the appropriate operating regimes required to fabricate bubbles with desired radii.

As one such illustration of the simplicity of Eq. (2.20), we can determine the bubble size reduction over a specific time interval. Figure 2.8 shows the dimensionless shrinkage time required for bubble shrinkage to $\hat{R}=0.01$ and $\hat{R}=0.1$ versus vacuum pressure for bubbles with different initial sizes. This information is useful from an engineering standpoint. For instance, this model informs us that we require a dimensionless time of $\hat{t}=30$ to shrink a bubble with initial size of $R_{0}=10 \mu \mathrm{m}$ to a final size of $R=0.1 \mu \mathrm{m}$ if a vacuum of $\widehat{P_{v}}=-0.6$ is applied, while we can achieve a final size of $R=1 \mu \mathrm{m}$ if a vacuum of $\widehat{P}_{v}=-0.5$ is applied. The amount of time required can be converted to the channel length and be used for designing a microfluidic shrinkage setup.

\subsection{Conclusion}

In this chapter, we characterize a microfluidic microbubble shrinkage technique that utilizes the gas permeability of PDMS microchannels and the permeability of bubble encapsulations, to shrink microbubbles. We develop a mathematical model, that predicts the size of the resulting microbubbles, and find a very good agreement between the model and experimental data, under different experimental conditions. We expect that this model, especially in the simplified closedform version, will find utility in the engineering and manufacturing of microbubbles of specific sizes.

Although our microfluidic bubble-shrinking device results in low volumes of gas surrounded by relatively large volume of liquid (i.e., low number of bubbles per volume of liquid), multiple techniques can be implemented to increase the throughput. These techniques include on-chip multiplexing of the bubble-generation orifices, ${ }^{140}$ reducing the width $(<5 \mu \mathrm{m})$ of the flow-focusing orifice using precise microfabrication techniques, ${ }^{128}$ and using an on-chip bubble separator to remove the carrier liquid and control the number of bubbles per liquid volume. ${ }^{141,142}$ Our modeling and experimental results reported in this chapter can also be used to better understand the physical 
mechanism behind vacuum-based bubble shrinkage, and enable the design of a device capable of reducing the bubble sizes down to order $1 \mu \mathrm{m}$ or less, thus potentially offering a sub-micron bubble generation technique. In such a device, one can predict the amount of vacuum pressure required for a specific type of lipid solution in order that a desired bubble size is collected at the outlet. Channel design optimization, investigation of other types of bubble encapsulations, and integration with submicron bubble characterization techniques offer future research directions to pave the way towards making inexpensive and easy-to-fabricate stabilized nanobubbles. 


\section{Expansion-Mediated Bubble Breakup in Microfluidics}

The work presented in this chapter is based on the following article, ${ }^{143}$ which is published in a peer-reviewed journal Physical Review Fluids, and is reproduced here with permission from the American Physical Society.

Salari, A.*, Xu, J.*, Kolios, M.C., and Tsai, S.S.H. Expansion-mediated breakup of bubbles and droplets in microfluidics. Phys. Rev. Fluids 5, 013602 (2020).

\section{Author's Contribution}

For the article presented here, the author wrote the majority of the manuscript, developed the dimensional modeling, designed the experiments, conducted the data analysis, and fabricated the devices. J. Xu performed the majority of the experiments, and collected the majority of the experimental data, and helped with discussing the results and writing the manuscript.

\subsection{Introduction}

When two immiscible fluids meet at a microfluidic junction, interfacial instabilities, such as Rayleigh-Plateau, causes one fluid to break into a series of dispersions - bubbles or droplets. ${ }^{144}$ Due to many applications that utilize bubbles and/or droplets, the formation strategies ${ }^{145,146}$ and breakup regimes ${ }^{147,148}$ of droplets and bubbles impact fields ranging from emulsion and colloid 
formation, ${ }^{90,121}$ bioagent encapsulation, ${ }^{149}$ polymerase chain reaction, ${ }^{150}$ ultrasound contrast agent production, ${ }^{151}$ and food processing. ${ }^{152}$ The primary droplets and bubbles, which are formed when the two immiscible fluids first meet in a microfluidic device, are also observed to break into secondary droplets and bubbles in various geometries, including at microfluidic Tjunctions, ${ }^{147,153,154}$ and constrictions. ${ }^{155}$ In all of these breakup cases, the droplet or bubble is physically obstructed, compressed, and then eventually broken into smaller droplets or bubbles, respectively.

One important dimensionless number describing the generation of bubbles and droplets in a microfluidic channel is the Capillary number, $C a=\mu v / \sigma$, where $\mu, v$, and $\sigma$ are liquid viscosity, liquid flow rate, and interfacial tension, respectively. Typically, the breakup of droplets and bubbles flowing in a microfluidic channel occurs at relatively low Capillary numbers, where, $\mathrm{Ca}$ $=O\left(10^{-2}\right) \cdot{ }^{148,156-159}$ Hashimoto et al. shows that in a system of droplets with sufficiently low Capillary number and low interfacial tension, $\sigma=O\left(10^{-1}\right) \mathrm{mN} \mathrm{m}^{-1}$, driven in a microfluidic HeleShaw cell (HSC), capillary and shear-driven instabilities induce the breakup of droplets. ${ }^{156} \mathrm{In}$ such an HSC configuration, droplets generated at a flow-focusing junction are delivered into an expanded channel that is fifty times wider than its height (so that the 2D dimensional flows in the HSC obeys Darcy's law, mathematically equivalent to the flow in a porous medium). The timescale required for these instabilities to evolve is relatively long, $t=O(10) \mathrm{ms},{ }^{156}$ compared to another regime in a similar geometry studied by Vecchiolla et al., which is shown to have a shorter breakup time-scale, $t=O(0.1) \mathrm{ms} .{ }^{160} \mathrm{In}$ the latter regime, however, bubbles split near the entrance of the expansion region, at a higher Capillary number, $C a=O\left(10^{-1}\right)$ and at a higher rate of $\sim 10,000$ bubbles per second. Vecchiolla $e t$ al. also show that bubbles can either flow undisturbed, break periodically into equal-sized daughter bubbles, or break irregularly depending on the value of Capillary number. They demonstrate that periodic asymmetric breakup, where the daughter bubbles are of unequal sizes, can only be achieved if an asymmetric geometry of the expansion region is employed. In all designs reported by Vecchiolla et al., the channel width at the expansion region increases at an angle less than $90^{\circ}$, and thus, as bubbles enter the expansion region, their travelling speed decreases gradually. In such a geometry, the competition between interfacial tension and the viscous stress imposed by the continuous flow appears to govern the periodic 
breakups, and thus, the Capillary number is the major dimensionless number in the power law relationship. ${ }^{160}$

Here, we use a flow-focusing microfluidic design, coupled with a sudden increase in channel width, to further study different flow regimes in expansion-mediated geometries, and quantify symmetric and asymmetric breakup of bubbles. In contrast to the geometry used by Vecchiolla et $a l$, in our microfluidic design, the channel width increases at $90^{\circ}$ at the expansion region, which causes the speed of the bubbles to decrease immediately once they enter the expansion region. This sudden increase in the channel width causes the effect of inertia to become critical, especially at the entrance of the expansion region. Therefore, we find that the inertial force-as quantified by the dimensionless Weber number, $\mathrm{We}$ - significantly contributes to determining the transition from non-breakup to breakup regimes. In addition to mono-disperse and bi-disperse output populations reported by Vecchiolla et al., we observe an additional regime, where the microfluidic system produces periodic tri-disperse populations. To better understand the breakup mechanism, we perform dimensional analyses to characterize the competition between governing physical effects, which are inertia, viscosity, and interfacial tension. Our finding suggests that the dimensionless Ohnesorge number, $O h$, defines the breakup transition from symmetric to asymmetric modes. We also ask the additional question of what happens when we flow different liquid droplets, instead of bubbles, into the expansion, and we find the same flow regimes as in our bubble experiments. Finally, we find that all of our experimental results follow a power law, $f^{0.5} \propto W e^{0.1} \mathrm{Ca}^{0.2}$, which precisely predicts when dispersions will breakup. The dimensionless theoretical framework presented here can be applied to other expansion-mediated geometries and various types of fluids, to help engineer multi-disperse populations for applications such as producing complex wet foams, ${ }^{161-163}$ and self-assembling hyperuniform materials. ${ }^{164,165}$

\subsection{Methods}

The microfluidic device we use for the generation of air microbubbles is shown in Figure 3.1a and $\mathrm{b}$, where the two flows meet at a flow-focusing cross-junction. The same geometry is used to generate droplets. The dispersed phase, which is a gas in bubble experiments and a liquid in droplet experiments, is supplied using a pressure regulator (Omega Engineering), and a pressure 
pump (Fluigent), respectively. The aqueous continuous phase is infused by a syringe pump (Harvard Instruments).
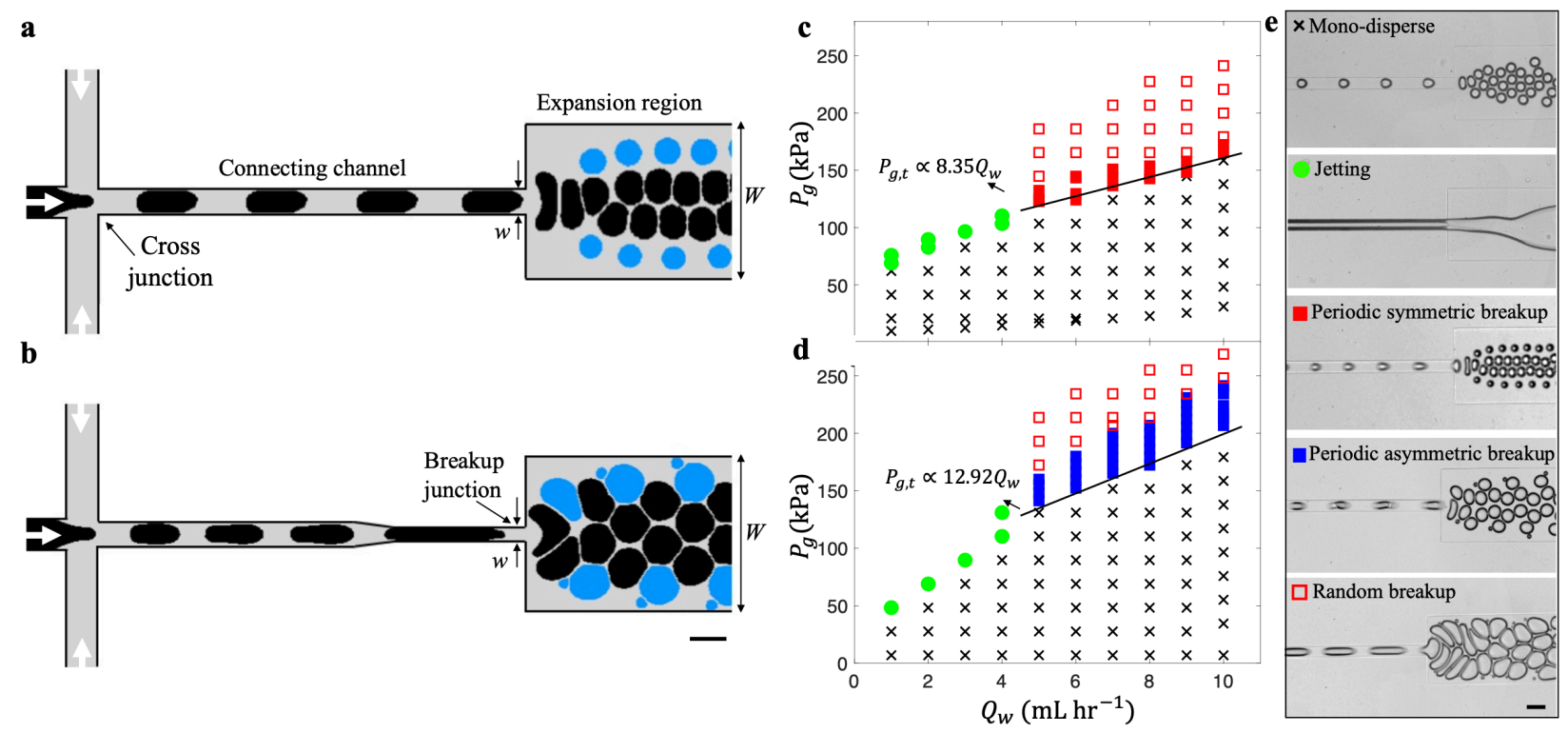

Figure 3.1 (a-b) Schematic diagram of the device showing a microfluidic flow-focusing geometry coupled with an expansion region at the breakup junction. First, air microbubbles (shown in black color) are generated at the flow-focusing cross-junction and then travel through the connecting channel, which leads to the expansion region. The two geometries used in this study have identical dimensions except at the breakup junction. In (a), the connecting channel has a uniform width $w=$ $40 \mu \mathrm{m}$. In (b), the connecting channel width tapers from $40 \mu \mathrm{m}$ to a width $w=20 \mu \mathrm{m}$ at the breakup junction, over a $100 \mu \mathrm{m}$ distance that begins $300 \mu \mathrm{m}$ upstream from the breakup junction. At specific values of certain geometrical and flow parameters, the microbubbles are squeezed at the entrance of the expansion region, and thus, periodically break into smaller daughter bubbles (shown in blue). $w$ and $W$ represent the widths of the breakup junction and expansion region, respectively. The continuous and dispersed phases are shown in gray and black colors, respectively. (c-e) Different flow regimes observed in our bubble generation experiment. Here, the geometry has the breakup junction width $w=40 \mu \mathrm{m}$ and the experiment uses two different aqueous solutions: (c) Aq-b1; (d) Aq-b2 (see Table 3.1). At an aqueous flow rate $Q_{w}>5 m L h r^{-1}$, increasing the gas pressure $P_{g}$ eventually leads to the transition from mono-disperse bubbles to periodic symmetric or asymmetric breakup, and then random breakup. (e) Microscope images of each flow regime in this experiment. The scale bars represent $50 \mu \mathrm{m}$. 
The aqueous continuous phase in our study consists of different mixing ratios of glycerol, deionized water, Pluronic F-68, and sodium dodecyl sulfate (SDS). We tune the viscosity and interfacial tension by adjusting the amount of glycerol and SDS in the aqueous solution, respectively. The viscosity is measured using a Cannon-Fenske Routine viscometer, and the interfacial tension is measured using the pendant drop method. ${ }^{120} \mathrm{We}$ use the following three different aqueous solutions for bubble generation experiments: Aq-b1 is a mixture of glycerol, DI water, and Pluronic F-68 at a 0.5:1:1 volume ratio, which has a liquid-air interfacial tension $\sigma=$ $41.9 \mathrm{mN} \mathrm{m}^{-1}$ and viscosity $\eta=3.23 \mathrm{mPa} \mathrm{s}$; Aq-b2 is the mixture of glycerol, DI water, and Pluronic F-68 at a 1:1:1 volume ratio, plus 1 wt. \% SDS, and has liquid-air interfacial tension $\sigma=$ $37.6 \mathrm{mN} \mathrm{m}^{-1}$, and viscosity $\eta=6.45 \mathrm{mPa} \mathrm{s}$; Aq-b3 is a mixture of glycerol, DI water, and Pluronic F-68 at a 1:1:1 volume ratio, and has liquid-air interfacial tension $\sigma=40.6 \mathrm{mN} \mathrm{m}^{-1}$, and viscosity $\eta=5.16 \mathrm{mPa}$ s. Table 3.1 shows a list of the aqueous solution abbreviations and their properties that we use in the bubble experiments. It also contains the properties of solutions that we use in the droplet experiments, which are mentioned later in the text.

We fabricate the microfluidic device using photolithography followed by soft lithography. ${ }^{166}$ All microfluidic channels have a height of $26 \mu \mathrm{m}$. The molded polydimethylsiloxane (PDMS) replicate of the microfluidic channels is then bonded to a glass slide by plasma treatment. Once dispersions are generated at the cross-junction, they travel through the connecting channel before entering the expansion region. The connecting channel is designed to be long $(850 \mu \mathrm{m})$ to ensure that the dispersion generation at the cross-junction does not interfere with breakup at the expansion region.

We design two microfluidic geometries that have identical cross-junction width, connecting channel length, and expansion region width, and differ only by the breakup junction geometry (Figure 3.1a and b). The first geometry has a uniform breakup junction width $w=40 \mu \mathrm{m}$ (Figure 3.1a). The second geometry has a breakup junction width $w=20 \mu \mathrm{m}$. The connecting channel width of this geometry tapers, at a constant angle, from $40 \mu \mathrm{m}$ to $w=20 \mu \mathrm{m}$ over a $100 \mu \mathrm{m}$ distance that begins $300 \mu \mathrm{m}$ upstream from the breakup junction (see Figure 3.1b). 
Table 3.1 List of the aqueous solutions and their properties used in our experiments.

\begin{tabular}{cccc} 
Experiment & Aqueous solution & Interfacial tension $\sigma\left(\mathrm{mN} \mathrm{m}^{-1}\right)$ & Viscosity $\eta(\mathrm{mPa} \mathrm{s})$ \\
& Aq-b1 & 41.9 & 3.23 \\
Air bubble & Aq-b2 & 37.6 & 6.45 \\
& Aq-b3 & 40.6 & 5.16 \\
\hline \multirow{3}{*}{ Dodecane droplet } & Aq-d1 & 8.61 & 3.23 \\
& Aq-d2 & 5.82 & 6.45 \\
& Aq-d3 & 9.52 & 5.16 \\
\hline \multirow{3}{*}{ Mineral oil droplet } & Aq-m1 & 1.83 & 15.41 \\
& Aq-m2 & 1.48 & 20.36 \\
& Aq-m3 & 2.84 & 21.98
\end{tabular}

\subsection{Results and Discussions}

In a typical cross-junction bubble generation microfluidic device, gas pressure, interfacial tension, and the aqueous solution flow rate and viscosity are the main parameters governing the size of the bubbles pinched off from the gas thread. ${ }^{12,167}$ In these systems, the output of the device is either mono- (or under specific conditions poly- ${ }^{168}$ ) disperse bubbles or jets (governed by nonlinear instabilities ${ }^{169,170}$ ). These regimes can also be observed in our bubble breakup experimental results. As shown in Figure 3.1c and d, at low aqueous solution flow rates $Q_{w}<$ $5 \mathrm{~mL} \mathrm{hr}^{-1}$ and relatively low gas pressure $P_{g}$, we observe mono-disperse bubbles, as expected. ${ }^{13,167}$ For a gas pressure $P_{g} \sim 50-100 \mathrm{kPa}$ the gas thread reaches the expansion region (also called jetting regime) and no bubble generation occurs.

However, as shown in Figure 3.1c and $\mathrm{d}$, at relatively high liquid flow rates $Q_{w}>$ $5 \mathrm{~mL} \mathrm{hr}^{-1}$, we observe a third regime at high gas pressures $P_{g}$, where mono-disperse bubbles generated at the cross-junction, breakup into two smaller daughter bubbles once they enter the expansion region (see Video B.1 and Video B.2). At this point increasing the gas pressure $P_{g}$ causes the periodic bubble breakup regime to transition into a random breakup regime. Here, the term "periodic" denotes the phenomenon of a constant number ratio of breakup to non-breakup bubbles at the expansion region (for example, one out of every three bubbles breaks into daughter bubbles). Random breakup refers to the case where this number ratio is not constant. Additionally, we observe both symmetric (i.e., roughly equal sized daughter bubbles) and asymmetric (i.e., unequal sized daughter bubbles) breakup. We find the experimental best fit transition lines $P_{g, t} \propto 8.35 Q_{w}$ 
and $P_{g, t} \propto 12.92 Q_{w}$, for the transition between mono-disperse to periodic regimes in Figure 3.1c and Figure 3.1d, respectively.

We note that the breakup regime studied here is different from the bifurcation of bubble sizes observed at a flow-focusing device, ${ }^{168}$ where at the orifice, the system produces sequences of bubbles with two (or more) different sizes. In such a system, bubbles of different sizes are pinched off from the gas thread, whereas, in our system, mono-disperse bubbles breakup at the expansion, regardless of where and how they are generated.

In both of our geometries, the liquid flow velocity in the expansion region is approximately an order of magnitude lower than the velocity in the connecting channel. The lower velocity dictates that once the bubbles enter the expansion region, they decelerate significantly, and mimic slow-moving obstacles that cause the flow streamlines to deviate to the sides of the expansion region. If the subsequent bubble enters the expansion region before the streamlines are relaxed to their original position, the subsequent bubble becomes squeezed, elongates, and breaks into daughter bubbles (see for example Figure 3.2a).
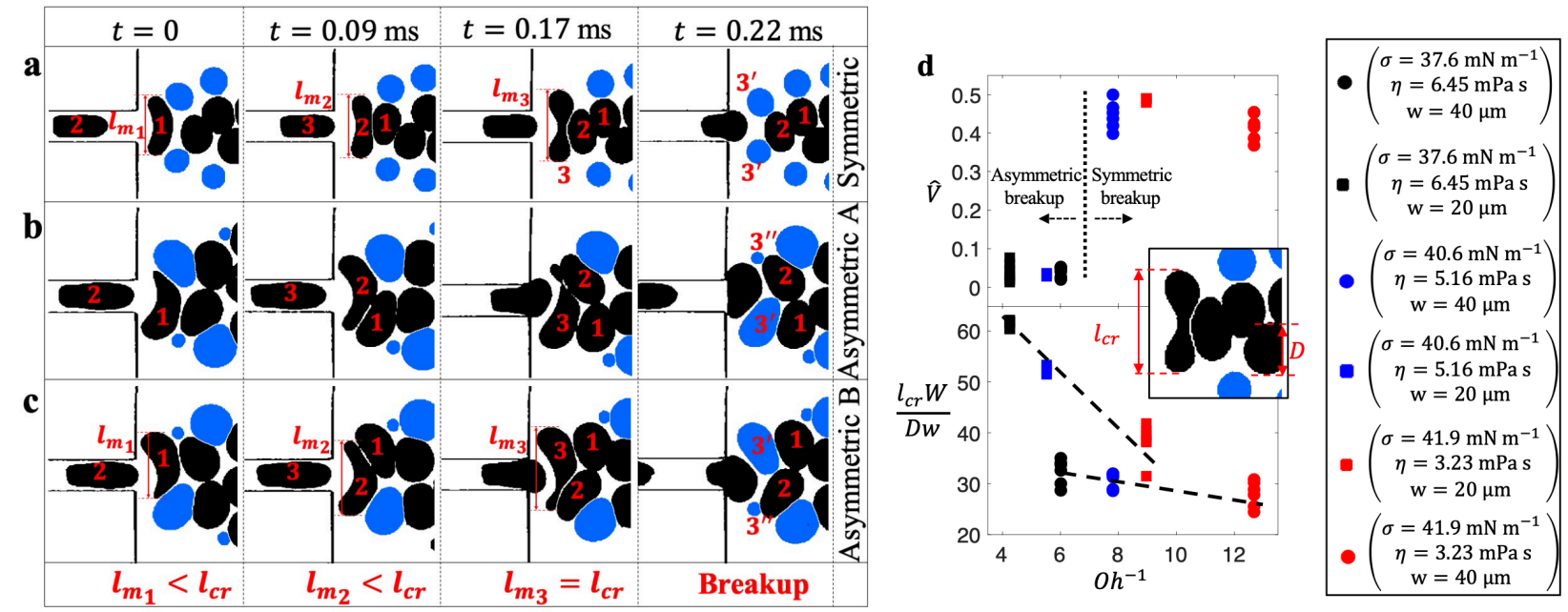

Figure $3.2(\mathrm{a}-\mathrm{c})$ Images of different modes of periodic breakup occurring at the entrance of the expansion region. Intact bubbles are shown in black, whereas daughter bubbles that result from breakup, are in blue. (a) The periodic symmetric mode, and (b, c) the periodic asymmetric mode. The asymmetric mode can be further categorized into two sub-modes A and B, which occur consecutively one after another, meaning that every asymmetric A sub-mode is followed by an 
asymmetric B sub-mode. As shown, the presence of the pre-arrived bubbles " 1 " and "2" is crucial for the breakup of the " 3 "rd bubble. Each bubble is elongated to its maximum length of $l_{m}$, once it enters the expansion region. In a sequence of three arriving bubbles, only one bubble is elongated to its critical length of $l_{c r}$, at which point breakup occurs. The images in (a) are from experiments that use Aq-b1, and in (b, c) Aq-b2 is used. (d) A plot of the volume ratio of the daughter bubbles (top graph), and dimensionless critical length immediately before breakup (bottom graph) versus the inverse Ohnesorge number $O h^{-1}$. The value of $O h^{-1}$ determines the periodic breakup mode. As shown, for each geometry, a decrease in $l_{c r} / D$ is observed by increasing $O h^{-1}$, suggesting that the effect of shear force becomes significant in asymmetric breakup. However, in the symmetric mode, shear appears to be a secondary dominant force. All data depicted in (d) are collected at the transition pressure of $P_{g, t}$, the point at which the mono-disperse regime changes to the periodic breakup regime (see Figure 3.1c and d).

All bubbles experience a high degree of shear and inertia at the entrance of the expansion region, which causes them to become elongated to a maximum length $l_{m}$ perpendicular to the average flow direction. At this position, if the pro-elongation inertial and shear forces overcome the interfacial tension force, the bubble will breakup. We denote the corresponding critical bubble length $l_{c r}$, as the measured length of the bubble right before breakup. Based on our observations, in a periodic breakup regime, the number of bubbles entering the expansion region between two successive breakups for all experiments is within the range of 2, where one of every two bubbles break, to 5 , when one of every five bubbles break.

Figure 3.2a-c shows an example sequence of bubble breakup for both symmetric and asymmetric modes, where the period is 3. As shown, no breakup occurs for bubbles labeled as "1" and "2" that have maximum length $l_{m}<l_{c r}$ as they restore to their original shape after moving downstream. This means that not all bubbles break. The presence of pre-arrived bubbles "1" and "2" at the expansion region is a key factor for the breakup of an arriving bubble " 3 ".

As shown in Figure 3.2a, bubble "2" temporarily (based on our observations, $\sim 10^{-2} \mathrm{~ms}$ ) blocks the path of the " 3 "rd bubble at the entrance of the expansion region. Thus, for this time 
period, the role of bubble "2" is physically similar to that of the channel wall in a T-junction geometry. ${ }^{147,153}$

In contrast to T-junctions, where asymmetrical channel dimensions lead to asymmetrical breakup, ${ }^{148,171}$ in our device, both symmetrical and asymmetrical breakup can occur in a symmetrical microchannel geometry. The type of breakup depends on the flow conditions. Asymmetric A and B regimes shown in Figure 3.2b and c, respectively, are the two sub-modes of asymmetric breakup that occur consecutively. Every asymmetric A sub-mode is followed by an asymmetric B sub-mode. The difference between these two sub-modes is the location of breakup. As shown in Figure 3.2b and c, the breakup location of bubble " 3 " is shifted towards one side of the expansion region, and the larger daughter bubble " 3 " " flows adjacent to bubble " 1 " while the smaller daughter bubble " 3 "” flows adjacent to bubble " 2 ".

Inspired by the observations of droplet breakup occurring at microfluidic Tjunctions, ${ }^{153,172,173}$ we hypothesize that in our system, the major force competition governing breakup is between the inertial force, which scales as $\rho v^{2} D^{2}$, and interfacial tension force, which scales as $\sigma D$. Here, $\rho, v$, and $D$ are the liquid density, average velocity at the connecting channel, and the (projected) initial diameter of the bubble, respectively. We note that the actual bubble diameter is different from the projected diameter because the bubble diameter, upon exiting the device, is larger than the channel height, and thus the bubble within the microchannel is squeezed from its top and bottom. Therefore, we calculate the actual volume of the bubbles using a mathematical approach that is explained elsewhere in the literature. ${ }^{121}$ Briefly, the volume of a discoid bubble is approximately equal to the volume of an undeformed bubble minus two caps. By measuring the discoid diameter, we can calculate the volume of the bubble.

To test our hypothesis, we define the Weber number as $W e=\rho v^{2} D \sigma^{-1}$ and map the values of $W e$ for the two geometries that correspond to the breakup regime. We observe that the breakup regime is $2<W e<8$ for the geometry with junction width $w=40 \mu \mathrm{m}$, and $10<W e<45$ for the geometry with junction width $w=20 \mu \mathrm{m}$. We are unable to run experiments for $W e>8$, and $W e>45$ in the two geometries, respectively, due to the large pressure at the tubing connections. 
Thus, we find the non-breakup to breakup critical Weber number $W e_{c r} \approx 2$ and $W e_{c r} \approx 10$ for the two geometries, respectively.

In our system, since mono-disperse bubbles are first generated upstream in the same microfluidic device, ensuring that $W e>W e_{c r}$ causes the generation of bubbles of specific size and at generation frequencies that can lead to periodic breakup. Thus, $W e>W e_{c r}$ is the only requirement for the breakup to occur in our system. If bubbles are first generated elsewhere, and then transferred into a microfluidic device equipped with an expansion geometry similar to ours, then there will be a minimum ejection rate (in our experiments it is within the range of $10-30$ $\mathrm{kHz}$ ), at which the bubbles are required to enter the expansion region, in addition to having $W e>$ $W e_{c r}$, to ensure that breakup occurs. This range ensures that the bubble enters the expansion region before the streamlines are relaxed to their original position. This is different from the case of breakup in T-junction geometries, where the ejection rate of bubbles does not play a significant role. In a hypothetical case of very low ejection rate (not shown here), the probability of breakup would be minimized. In such a case, $W e_{c r}$ would be much higher than the values reported here, and the symmetric breakup would be dominant over the asymmetric breakup. Maintaining a small value of the design ratio, $W / w$, would also help in achieving breakup at lower $W e_{c r}$. In contrast, assuming a very high ejection rate of bubbles, the breakup regime would still be achievable at a $W e_{c r}$ value that is in the same range of those reported here. At this extreme case, the breakup would have to be asymmetric, since the expansion region would be fully occupied by bubbles, and the arriving bubbles would be pushed to the sides of the expansion region. ${ }^{174}$

In order to characterize symmetric and asymmetric periodic breakup modes, we define the Ohnesorge number, $O h=\mu / \sqrt{\rho \sigma w}$, which compares the strength of viscosity to inertia and interfacial tension. Our results show that when $O h^{-1}<7$, bubbles entering the expansion region move periodically to the sides of the expansion. This is due to an abrupt decrease in the liquid speed that causes the bubbles to slow down and push against each other (Figure 3.2b and c). If a bubble becomes squeezed and the shear stress overcomes the interfacial tension, it will stretch beyond $l_{c r}$, and eventually break asymmetrically into two daughter bubbles with unequal sizes. This is shown in Figure 3.2d, when $O h^{-1}<7$, and for all different aqueous solutions, the daughter 
bubbles' volume $\widehat{V}<0.1$, where the dimensionless volume $\widehat{V}$ is the volume ratio of the smaller daughter bubble to the intact bubble.

Asymmetric breakup can only occur if the inertial force is relatively low, whereas, at high inertial forces, the breakup becomes more symmetric, meaning that bubbles break into two equal size daughter bubbles. In this situation, the flow inertia is strong enough to push the preceding bubbles away from the breakup junction, prior to the arrival of the subsequent bubble. This causes the breaking bubble to experience two equal forces, and thus, the bubble breaks into two roughly equal sized daughter bubbles. This observation corresponds to $\widehat{V} \approx 0.4-0.5$, when $O h^{-1}>7$ (Figure 3.2d). Therefore, we define a critical $O h^{-1}$ number $O h_{c r}^{-1}=7$ that roughly separates symmetric and asymmetric breakup modes. This finding enables us to design a tri-disperse bubble generation device, without adding asymmetry to the geometry as in Vecchiolla et al. ${ }^{160}$

Our observations also reveal that the critical bubble length $l_{c r}$ is in the range of $1.7<$ $l_{c r} / D<2.4$, and depends on the Ohnesorge number, $O h$, scaling as $O h \propto l_{c r} W / D w$. As shown in lower graph of Figure 3.2d, in both geometries, the dimensionless critical length $l_{c r} / D$ of an asymmetric breakup is higher than in symmetric breakup. The value of $l_{c r} / D$ is directly proportional to the magnitude of viscous stress. This suggests that, in asymmetric breakup, shear stress contributions are comparable to, and in some cases more significant than, inertial contributions. In symmetric breakup, however, inertia is the dominant contributor. Therefore, we also need to consider the Capillary number in order to fully characterize the breakup over different experimental conditions. It should be noted that for larger values of $W / w$, a larger inertial force is required to trigger the breakup regime. This explains why $W e_{c r}$ is lower in experiments with the geometry, $w=40 \mu \mathrm{m}$, than those with $w=20 \mu \mathrm{m}$. Our observations also suggest that once the periodic breakup regime begins, geometries with smaller aspect ratio, $W / w$, induce a more stable breakup, and thus the transition between symmetric and asymmetric modes is less dependent on the flow properties. Furthermore, Figure 3.2d reveals that an increase in the bubble size will shift the breakup regime in each geometry towards the symmetric mode, as opposed to the breakup of smaller bubbles that will be shifted towards the asymmetric mode. 
Figure 3.3 shows the bubble size distributions at the outlet of our device with a maximum bubble production rate of $1.6 \times 10^{5} \mathrm{~s}^{-1}$. When no breakup occurs, only a mono-disperse size distribution is obtained (Figure 3.3a). By only adjusting the flow rate and gas pressure, however, we are able to achieve bubble breakup at the expansion region to yield a multi-disperse population at the outlet (see Figure $3.3 \mathrm{~b}$ and c). 


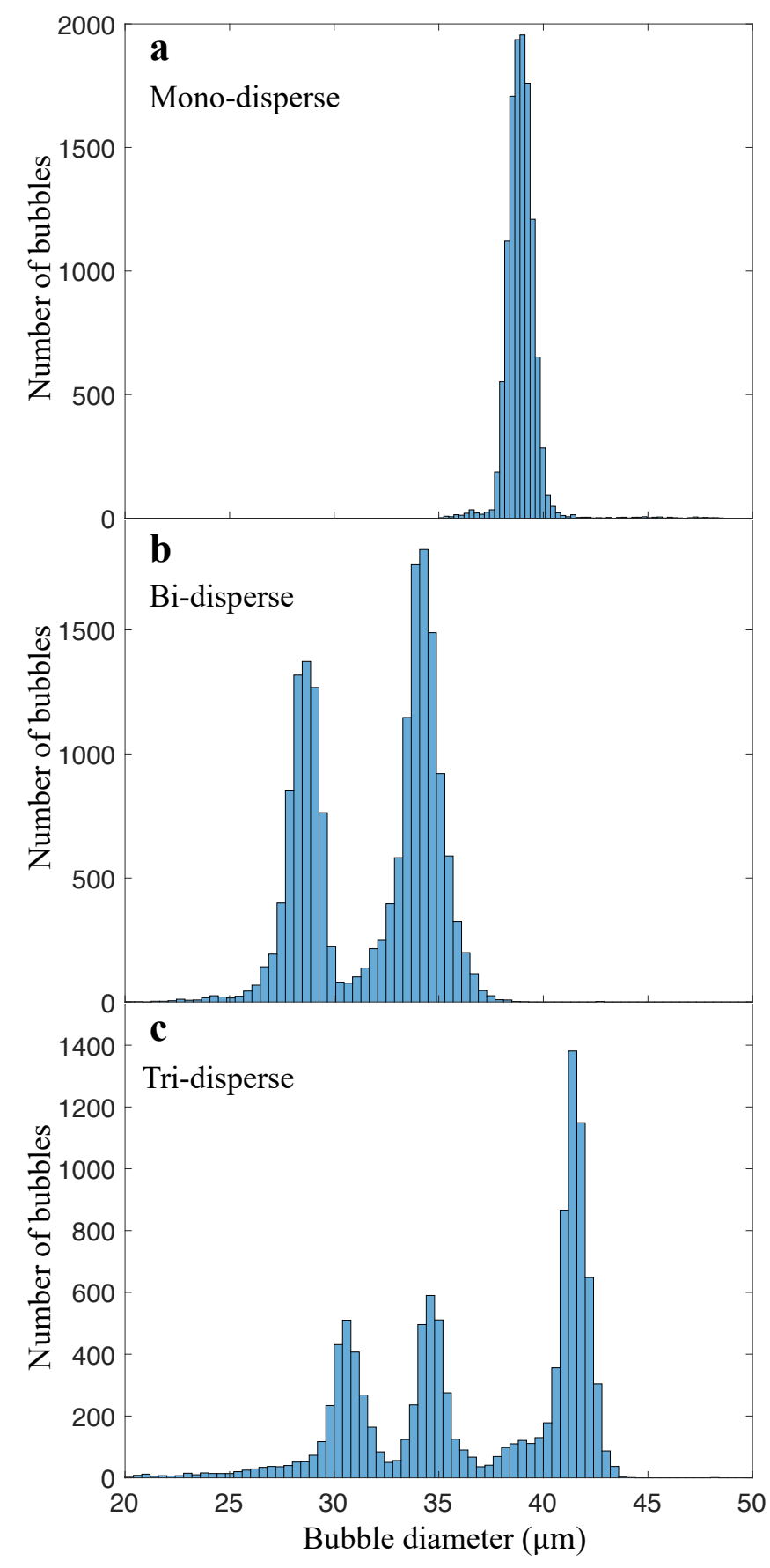

Figure 3.3 Different output populations obtained in our bubble generation experiments. Here, the geometry with the breakup junction width $w=40 \mu \mathrm{m}$ and continuous phase Aq-b1 is used. (a) $P_{g}=100 \mathrm{kPa}, Q_{w}=5 \mathrm{~mL} \mathrm{hr}{ }^{-1}$; (b) $P_{g}=160 \mathrm{kPa}, Q_{w}=10 \mathrm{ml} \mathrm{hr}^{-1}$; (c) $P_{g}=180 \mathrm{kPa}$, $Q_{w}=10 \mathrm{~mL} \mathrm{hr^{-1 }}$. As shown, using a single geometry, we obtain (a) mono-disperse, (b) bidisperse, or (c) tri-disperse populations only by controlling the aqueous phase flow rate and gas pressure. In (b) and (c), one in every three bubbles breaks. Therefore, the number of intact bubbles 
in (b) is approximately equal to the number of daughter bubbles, and in (c) the number of intact bubbles is approximately two times greater than the number of daughter bubbles.

It is known that the mechanisms governing the formation of bubbles and droplets in microfluidic devices are similar, ${ }^{14,145}$ so we hypothesize that expansion-mediated breakup mechanisms may also be similar. We thus design analogous experiments that use oil, i.e., dodecane and mineral oil, as the dispersed phase, and the same continuous phase as in the bubble experiments, albeit with different amounts of added glycerol. By changing the glycerol ratio in the continuous phase, we study the droplet breakup over a range of different viscosities.

We use aqueous solutions Aq-d1 with liquid-liquid interfacial tension $\sigma=8.61 \mathrm{mN} \mathrm{m}^{-1}$, Aq-d2 with liquid-liquid interfacial tension $\sigma=5.82 \mathrm{mN} \mathrm{m}^{-1}$, and Aq-d3 with liquid-liquid interfacial tension $\sigma=9.52 \mathrm{mN} \mathrm{m}^{-1}$ for the dodecane droplet experiments. In mineral oil droplet experiments, we use the following aqueous solutions: Aq-m1, is a mixture of glycerol, DI water, and Pluronic F-68 at a 3:1:1 volume ratio, which has a liquid-liquid interfacial tension $\sigma=$ $1.83 \mathrm{mN} \mathrm{m}^{-1}$ and viscosity $\eta=15.41 \mathrm{mPa}$; Aq-m2 is the mixture of glycerol, DI water, and Pluronic F-68 at a 4:1:1 volume ratio, plus $1 \mathrm{wt}$ \% SDS, and has a liquid-liquid interfacial tension $\sigma=1.48 \mathrm{mN} \mathrm{m}^{-1}$, and viscosity $\eta=20.36 \mathrm{mPa} \mathrm{s}$; Aq-m3 is a mixture of glycerol, DI water, and Pluronic F-68 at a 4:1:1 volume ratio, and has a liquid-liquid interfacial tension $\sigma=2.84 \mathrm{mN} \mathrm{m}^{-1}$, and viscosity $\eta=21.98 \mathrm{mPa}$. These properties are listed in Table 3.1.

In Figure 3.4a-b, when the liquid flow rate $Q_{w}>0.1 \mathrm{~mL} \mathrm{hr}^{-1}$, increasing $P_{g}$ causes droplets to transition from mono-disperse to periodic breakup. Here, mono-disperse oil droplets generated at the cross-junction, breakup into two smaller daughter droplets once they enter the expansion region (see Video B.3 and Video B.4). At this point, continuing increase of oil injection pressure $P_{g}$ causes the periodic droplet breakup regime to enter into a random breakup regime. Comparing Figure 3.1c-d and Figure 3.4a-b, we can observe identical flow regimes and similar transitions between different regimes in both bubble and droplet experiments. 

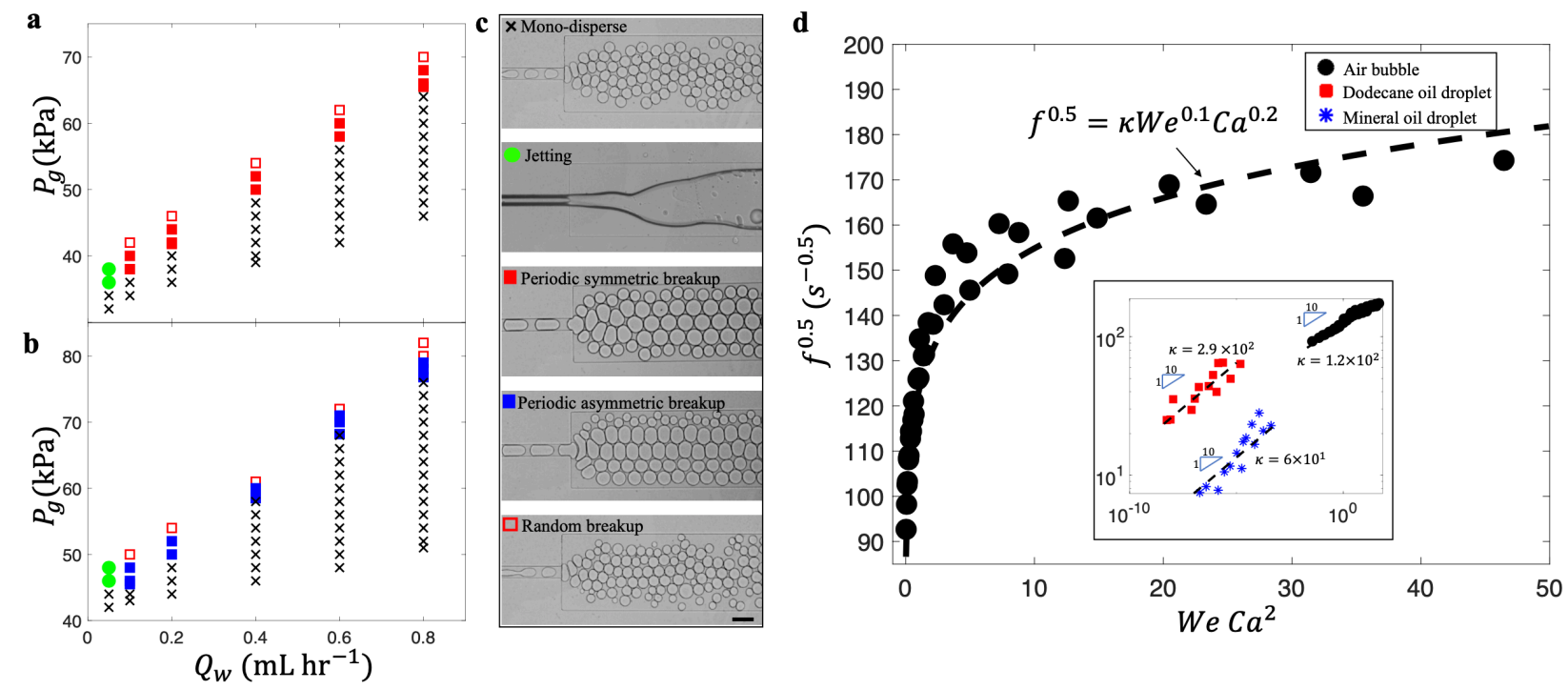

Figure 3.4 $(\mathrm{a}-\mathrm{c})$ Different flow regimes observed in our dodecane droplet generation experiment for the geometry of breakup junction width $w=40 \mu \mathrm{m}$ and two different aqueous solutions: (a) Aq-d1; (b) Aq-d2 (see Table 3.1). To be consistent with the results presented in Figure 3.1c and d, here, we report the dispersed phase pressure versus continuous phase flow rate. At an aqueous flow rate $Q_{w}>0.1 \mathrm{~mL} \mathrm{hr} r^{-1}$, increasing the oil pressure $P_{g}$ leads to the transition from monodisperse bubbles to periodic symmetric or asymmetric breakup, and then random breakup. (c) Microscope images of each flow regime in this experiment. Droplet breakup regimes exhibit similar trends of mono-disperse, periodic symmetric, and asymmetric breakup that are observed in bubble breakup experiments (see Figure 3.1c and d). (d) The frequency at which dispersions arrive at the expansion region, $\mathrm{f}$, versus the product of Weber number and the squared Capillary number, $W e C a^{2}$. Here, the dashed curve $\kappa W e^{0.1} \mathrm{Ca}^{0.2}$ is fitted to the experimental data points obtained from bubble experiments. The inset shows a $\log -\log$ plot of $f$ versus $W e C a^{2}$, where the dashed line shows the scaling, $f^{0.5}=\kappa W e^{0.1} \mathrm{Ca}^{0.2}$. All data depicted here are collected at the transition pressure of $P_{g, t}$, at which point the mono-disperse regime changes to periodic breakup regime (see Figure 3.1c and $\mathrm{d}$ and Figure 3.4a and b). The proportionality constant $\kappa$ for bubble, dodecane oil droplet, and mineral oil droplet experiments is $1.2 \times 10^{2}, 2.9 \times 10^{2}$, and $6.0 \times 10^{1}$, respectively. The bubble and droplet experimental results show a good agreement with the scaling, $f^{0.5} \propto W e^{0.1} \mathrm{Ca}^{0.2}$. The scale bar represents $50 \mu \mathrm{m}$. 
In our system, in addition to the fluid properties and microchannel design, dispersed phase inlet pressure and the continuous phase flow rate are the main design parameters that control the generation of either mono-disperse, bi-disperse (i.e., the result of periodic symmetric breakup), or tri-disperse microbubbles and droplets (i.e., the result of periodic asymmetric breakup).

In our experiments, the transition pressure $P_{g, t}$, at which the dispersion behavior moves from the mono-disperse regime to the periodic breakup regime, has a linear relationship with the continuous phase flow rate (see Figure 3.1c and d, and Figure 3.4a and b). Since dispersions are generated at a cross-junction in our device, this linear relationship implies that, the inlet pressure of dispersed phase is related to the frequency at which the dispersions arrive at the expansion region, as $P_{g, t} \propto f^{0.5} .^{13}$ This is also supported by our observations.

As shown in Figure 3.4d, we find experimentally, that this frequency follows the scaling $f^{0.5}=\kappa W e^{0.1} \mathrm{Ca}^{0.2}$, where $\kappa=1.2 \times 10^{2}, 2.9 \times 10^{2}$, and $6.0 \times 10^{1}$ for the bubble, dodecane oil droplet, and mineral oil droplet data points, respectively. This scaling law is reinforced when the data points are plotted on a log-log graph (inset of Figure 3.4d). Here, we change the variables $v$, $\mu$, and $\sigma$ in our experiments, and since $W e^{0.1} C a^{0.2}$ scales as $v^{0.4}, \mu^{0.2}$, and $\sigma^{-0.3}$, the relative importance of each parameter can be inferred. The variation between the pre-factor $\kappa$ associated with the data from bubble and droplet experiments may be attributed to the differences in dispersed phase properties (e.g., viscosity contrast of the fluids) ${ }^{175}$ in the bubble, dodecane oil droplet, and mineral oil droplet experiments. Experiments with fluids having a wider range of properties would be required to fully characterize the $\kappa$ values and can be investigated in future studies.

Figure 3.4d reveals that for both droplets and bubbles, the transition into the breakup regime is highly dependent on the continuous phase flow velocity. This is a major difference between the expansion-mediated and T-junction induced breakup phenomena. However, the competition between the viscous force, which promotes breakup, and the interfacial tension force, which inhibits breakup, is similar in both expansion-mediated and T-junction systems. ${ }^{148,153,175}$ Figure $3.4 \mathrm{~d}$ also shows that, for relatively small values of $W e \mathrm{Ca}^{2}$, the transition into the breakup regime becomes almost independent of the frequency of dispersions arriving at the expansion region, 
whereas, for relatively large values of $W e C a^{2}$, the transition is governed mainly by the magnitude of $f^{0.5}$.

\subsection{Conclusions}

In this chapter, we extend the idea of formation multi-disperse bubbles at a microfluidic expansion, ${ }^{160}$ by performing dimensional analyses to quantitatively identify the main physical parameters governing this phenomenon. This microchannel expansion breakup regime is based on bubble or droplet induced obstruction occurring in a microfluidic expansion region. We find that the physical breakup mechanisms of both bubbles and droplets in this regime are identical. We also define and experimentally fit the relevant dimensionless numbers, and control the output of the device, to produce mono-disperse, bi-disperse, and tri-disperse bubble or droplet populations without modifying the microfluidic geometry. We believe the results presented in this chapter are valuable for a wide range of audiences in the fields of bubble/droplet-based microfluidics for various applications in medicine, ${ }^{107}$ science, ${ }^{176}$ and technology. ${ }^{152,165,177}$ 


\section{Nanobubble Generation via Controlled Shrinkage of Microbubbles}

The work presented in this chapter is based on the following manuscript, which has not been published yet.

Xu, J., Salari, A., Wang, Y., He, X., Kerr, L, Darbandi, A., Kolios, M.C., Yuen, D., de Leon, A.C., Exner, A.A., and Tsai, S.S.H. Controlled shrinkage enabled microfluidic nanobubbles. In preparation.

\section{Author's Contribution}

For the article presented here, the author designed and conducted the proof-of-concept experiments, and fabricated the microfluidic devices, before J. Xu took the lead for the rest of the project. Throughout the project, the author actively helped with discussing the results and commenting on the manuscript. J. Xu led the experiments and wrote the manuscript. Y. Wang and $\mathrm{X}$. He performed the in vitro and in vivo experiments. L. Kerr helped with the bubble shrinkage experiments and resonant mass measurements. A. Darbandi performed the TEM characterizations. D. Yuen contributed to the design of the in vivo experiments and provided animal samples. A. C. de Leon and A. A. Exner assisted in developing the lipid solution protocols and bulk nanobubble generation experiments. 


\subsection{Introduction}

Nanobubbles play an increasingly important role in different research scenarios. ${ }^{178-180}$ One significant application of nanobubbles is in clinical diagnostics, ${ }^{181}$ where nanobubbles are introduced into blood flow as ultrasound contrast agents. In contrast to microbubbles, ${ }^{12}$ nanobubbles are much smaller than red blood cells. Their small size avoids vessel blockage and enables passage through endothelial barriers in tumors. ${ }^{181}$ According to previous studies, ${ }^{182}$ phospholipid-shell nanobubbles show excellent contrast enhancement.

However, current methods for producing nanobubbles have various limitations. High frequency mechanical agitation at the gas/water interface of a surfactant solution is the most commonly used method to produce a bulk nanobubble solution for ultrasound imaging applications. ${ }^{183}$ While simple and effective, this protocol produces a poly-disperse mixture of microbubbles and nanobubbles, ${ }^{184}$ which requires further centrifugation for separation.

Solvent exchange techniques, ${ }^{185}$ which are used to generate surface nanobubbles, rely on the difference of gas solubility in water versus in ethanol to precipitate gas out of the solution and form nanobubbles. This technique lacks the ability to sustain production, and thus is limited to static and qualitative surface characterization studies. A similar problem exists in the method of degassing and temperature change, ${ }^{186}$ which relies on the differences in gas solubility corresponding to pressure or temperature changes. The electrochemical method achieves stable generation of nanobubbles, ${ }^{187}$ however, the gases must be $\mathrm{H}_{2}$ or $\mathrm{O}_{2}$, and the method cannot be applied to electrochemically inert gases. Plasmonic nanobubbles generated in tissues in-situ, is a recent development of nanosurgery and detection. ${ }^{188}$ This method requires the delivery of nanoparticle clusters and follow-up laser perturbation to generate nanobubbles, which only have a lifetime of around several milliseconds, rendering it a complex and inefficient protocol.

Microfluidics has been widely used as a state-of-the-art tool to customize the size of monodisperse microbubbles with a high throughput. ${ }^{189}$ Generally, the size of a bubble generated in a microfluidic flow focusing geometry is proportional to the size of the orifice, gas flow rate, and interfacial tension, and inversely related to the liquid flow rate and viscosity. ${ }^{14}$ However, due to 
the resolution limitations of soft lithography ${ }^{190}$ the minimum size of bubbles produced by microfluidics is still far above the nanoscale, at around a few microns.

A microfluidic method that generates size-tunable and mono-disperse nanobubbles would overcome the current manufacturing limits of microfluidically-made bubbles, and potentially transform nanomedicine by providing reproducible mono-disperse and robust nanobubbles. Using such a method to produce nanometer ultrasound contrast agents would improve the contrast enhanced ultrasound images, and potentially pave the way for ultrasound molecular imaging. Microbubbles are intravascular contrast agents, but nanobubble contrast agents could in principle bind to target cells directly. This may result in more accurate monitoring of diseases, for example, the non-invasive monitoring of tumor progression. ${ }^{191}$

Moreover, the ability to produce mono-disperse nanobubbles for drug delivery would allow significantly improved dose precision. Therapeutic agents can be loaded on, or conjugated to, the nanobubble interfaces, and are then released via ultrasound-induced cavitation. Size of bubbles in a mono-disperse population of drug-conjugated nanobubbles produced via microfluidics could be tuned to maximize the nanobubble response to the ultrasound excitation frequency, to optimize the efficiency of drug delivery. However, despite the promise of mono-disperse nanobubbles in medicine, to date, there are no reports of the generation of size-tunable, mono-disperse sub-micron bubbles using microfluidics or nanofluidics.

\subsection{Results and Discussions}

Here, we describe a microfluidic approach to first generate mono-disperse microbubbles, then shrink the microbubbles into mono-disperse nanobubbles. Our technique is inspired by earlier work $^{90,94,192}$ showing that dispersions (i.e., emulsions and bubbles) that partially contain components highly-soluble in the continuous phase, can shrink significantly as the soluble component leaves the dispersions and dissolves in the continuous phase. In our previous microfluidic bubble shrinkage platform, the final size of the shrunk bubbles can be tuned by controlling design parameters including gas core solubility in the liquid phase, saturation level of the liquid phase, and shell permeability. ${ }^{94}$ Following this methodology, here, we design a gas 
mixture of two components: a high aqueous phase solubility gas, and a low aqueous phase solubility gas. We choose nitrogen $\left(\mathrm{N}_{2}\right)$ as the more soluble gas component, and octafluoropropane $\left(\mathrm{C}_{3} \mathrm{~F}_{8}\right)$ as the less soluble component. $\mathrm{C}_{3} \mathrm{~F}_{8}$ is one of the commercially-used gas cores in ultrasound contrast agents. ${ }^{193}$

In our system, nanobubbles are generated using a conventional PDMS microfluidic device equipped with a classical flow focusing geometry located upstream to a long serpentine channel (Figure 4.1a). The mixture of $\mathrm{N}_{2}$ and $\mathrm{C}_{3} \mathrm{~F}_{8}$, introduced through the gas inlet, is pinched off at the orifice due to the viscous forces exerted by the aqueous phase. Since the aqueous phase is a mixture of lipid in water, lipid shelled microbubbles are generated at the orifice. The water-soluble $\mathrm{N}_{2}$ component in the gas core of microbubbles diffuses into the surrounding aqueous phase, which initiates the shrinkage of the microbubbles, while most of the water-insoluble $\mathrm{C}_{3} \mathrm{~F}_{8}$ component remains undissolved inside the shrunk bubbles. By tuning both the fraction of $\mathrm{C}_{3} \mathrm{~F}_{8}$ in the gas mixture, and the flow parameters, we can shrink microbubbles down to nanobubbles of a controllable size. Theoretically, this method can also be applied to the production of nanobubbles with other gas core compositions.
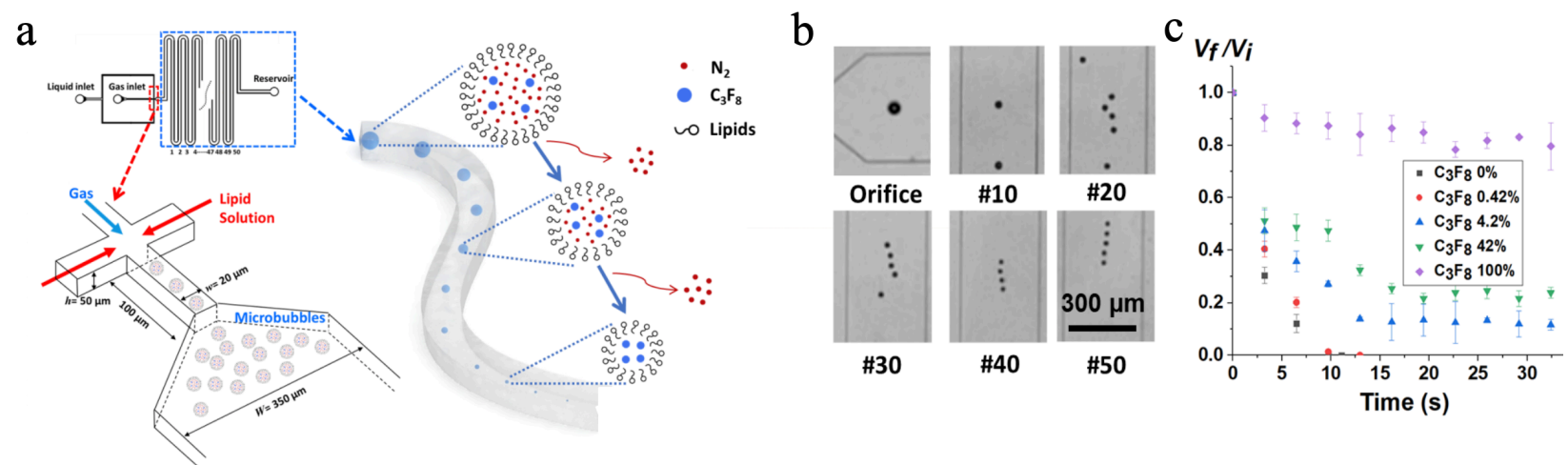

Figure 4.1 (a) Schematic diagram of the microfluidic nanobubble generator featuring a microbubble generation platform with a serpentine shrinkage channel. There are 50 turns in the serpentine channel, and the total serpentine channel length is $620 \mathrm{~mm}$; Dimensions of the crossjunction are: orifice width $\mathrm{w}=20 \mu \mathrm{m}$, channel height $\mathrm{h}=50 \mu \mathrm{m}$, and serpentine channel width $\mathrm{W}$ $=350 \mu \mathrm{m}$; Dissolution of $\mathrm{N}_{2}$ from the mixed gas core microbubbles leads to the shrinkage of the microbubbles down to the nanoscale. Insoluble $\mathrm{C}_{3} \mathrm{~F}_{8}$ constitutes the final equilibrium gas core of nanobubbles. (b) Bright-field images of the shrinkage of $4.2 \mathrm{wt} \% \mathrm{C}_{3} \mathrm{~F}_{8}$ in air microbubbles within 
the serpentine channel. The channel numbers, $\# 10, \# 20, \# 30, \# 40$, and $\# 50$, represent the number of times that the serpentine channel has turned. Each turn has a distance of $12 \mathrm{~mm}$ and thus the channel number indicates the distance from the bubble generation orifice. A longer distance corresponds to a longer travelling time. (c) A plot of the ratio of the normalized final bubble volume $V_{f} / V_{i}$, versus the dissolution time of the bubbles. Here, $V_{f}$ and $V_{i}$ are the final and initial bubble volume, respectively. All of the initial bubble volumes of different gas compositions are kept approximately constant. As shown, a decrease in $V_{f} / V_{i}$ is observed by decreasing the mass percent of $\mathrm{C}_{3} \mathrm{~F}_{8}$ in the gas mixture. Each data point shows the average of 3 measurements, and the error bars represent one standard deviation.

We obtain a maximum nanobubbles generation frequency of $10^{5} \mathrm{~s}^{-1}$, with a final bubble concentration of $\sim 10^{8} \mathrm{~mL}^{-1}$. Importantly, this method generates nanobubbles without significant changes to typical microfluidic bubble preparation methods, and without needing to improve the manufacturing resolution of microfluidic devices. This approach also provides a high-throughput production of nanobubbles.

The shrinkage process of microbubbles can be tuned by controlling the fraction of $\mathrm{C}_{3} \mathrm{~F}_{8}$ in the gas mixture. With a lab-made gas mixture of $4.2 \mathrm{wt} \% \mathrm{C}_{3} \mathrm{~F}_{8}$, we observe a significant decrease in microbubbles size along the serpentine channel (Figure 4.1b). By adjusting the fraction of $\mathrm{C}_{3} \mathrm{~F}_{8}$ in the mixture, we can control the shrinkage of the nanobubbles (Figure 4.1c). We note that this method is theoretically applicable to all gas mixtures comprised of multiple components of different solubility. By selecting appropriate gases and controlling their proportions, we can customize microbubbles or nanobubbles of specific gas core and size. 

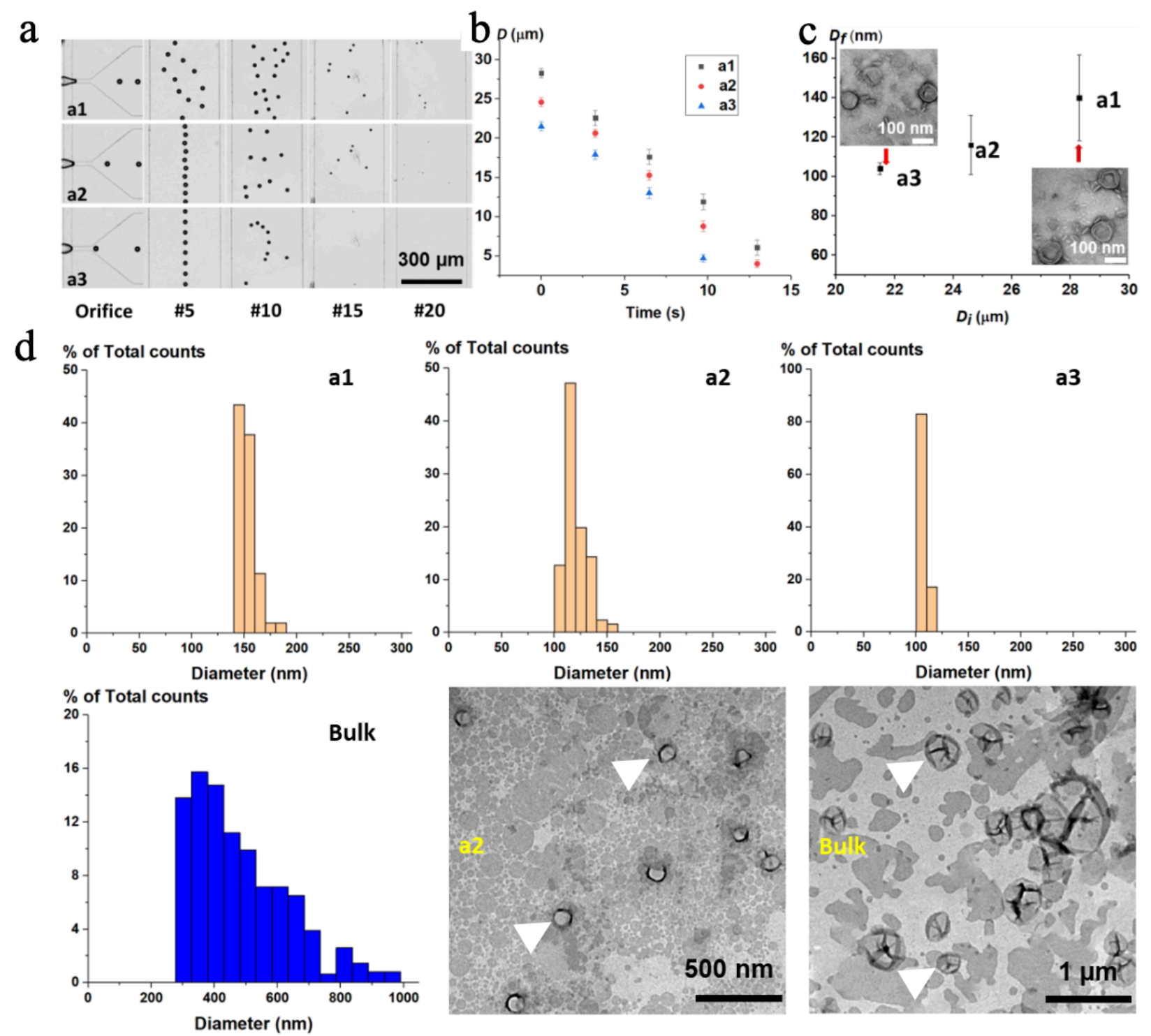

Figure 4.2 Shrinkage of microbubbles of different initial sizes, with the gas composition kept constant. (a) Bright-field images of different initial size microbubbles along the serpentine channel. The leftmost images are taken at the orifice, and the labels $\# 5, \# 10, \# 15$, and $\# 20$ correspond to the serpentine turn number. a1, a2, and a3 are the initial bubble diameters, $D_{i}=28.3$ $\mu \mathrm{m}, 24.6 \mu \mathrm{m}$, and $21.5 \mu \mathrm{m}$, respectively. (b) A plot of bubble diameter $D$ versus evolution time. (c) A plot of bubble final diameter $D_{f}$ versus initial diameter $D_{i}$. (d) Size measurements of final nanobubbles of a1, a2, and a3 by RMM and morphology characterizations by TEM. In TEM images, the white arrows point to individual bubbles as examples of monodisperse bubbles in MFNBs and polydisperse bubbles in Bulk-NBs. Each size measurement was repeated three times. In these experiments an industrial gas mixture of $0.17 \mathrm{wt} \% \mathrm{C}_{3} \mathrm{~F}_{8}$ in $\mathrm{N}_{2}$ is used. Bulk nanobubbles are 
taken from the $1 \mathrm{~mL}$ bottom layer of a $3 \mathrm{~mL}$ initial bulk nanobubbles solution, made by high frequency mechanical agitation, after centrifugation at $500 \mathrm{rpm}$ for 2 minutes, based on a modified version of a previously reported protocol. ${ }^{194,195}$

We also tune the shrinkage process of microbubbles by controlling their initial sizes, which is accomplished by adjusting the gas pressure. The evolution of microbubbles along the serpentine channel is shown in Figure 4.2a. We observe that the microbubbles of smaller initial size (e.g., $21.5 \mu \mathrm{m}$ diameter) shrink faster than larger microbubbles (Figure 4.2b). This phenomenon is in agreement with theoretical expectations, since smaller microbubbles experience larger Laplace pressure, which helps drive the gas molecules into the liquid.

We further confirm this trend by measuring the resulting nanobubbles using resonant mass measurement (RMM) technology (Archimedes, Malvern Instruments), the results of which are also supported by TEM imaging. The nanobubbles resulting from larger initial diameter microbubbles display a larger final diameter after gas dissolution (Figure 4.2c). We choose RMM as the primary measurement technique because it is the only method ${ }^{195}$ capable of distinguishing between nanobubbles of positive buoyancy ${ }^{196,197}$ and lipid particles or surfactant aggregates of negative buoyancy. In contrast, characterizations based on dynamic light scattering cannot distinguish between these two groups.

We use RMM to measure the size distributions of our microfluidically generated nanobubbles (MF-NBs) and bulk nanobubbles (Bulk-NBs) made by the mechanical agitation method (Figure 4.2d). Bulk-NBs-1 are diluted 1,000 times from Bulk-NBs-2 to match the concentration of MF-NBs. MF-NBs exhibit better monodispersity (a1, a2 and a3 in Figure 4.2d) than the Bulk-NBs, even after post-processing by centrifugation. We note that the measurements here are near the limits of detection of RMM, which likely truncates the size distribution measured. Nevertheless, TEM experiments also show better monodispersity in MF-NBs (Figure 4.2d). 


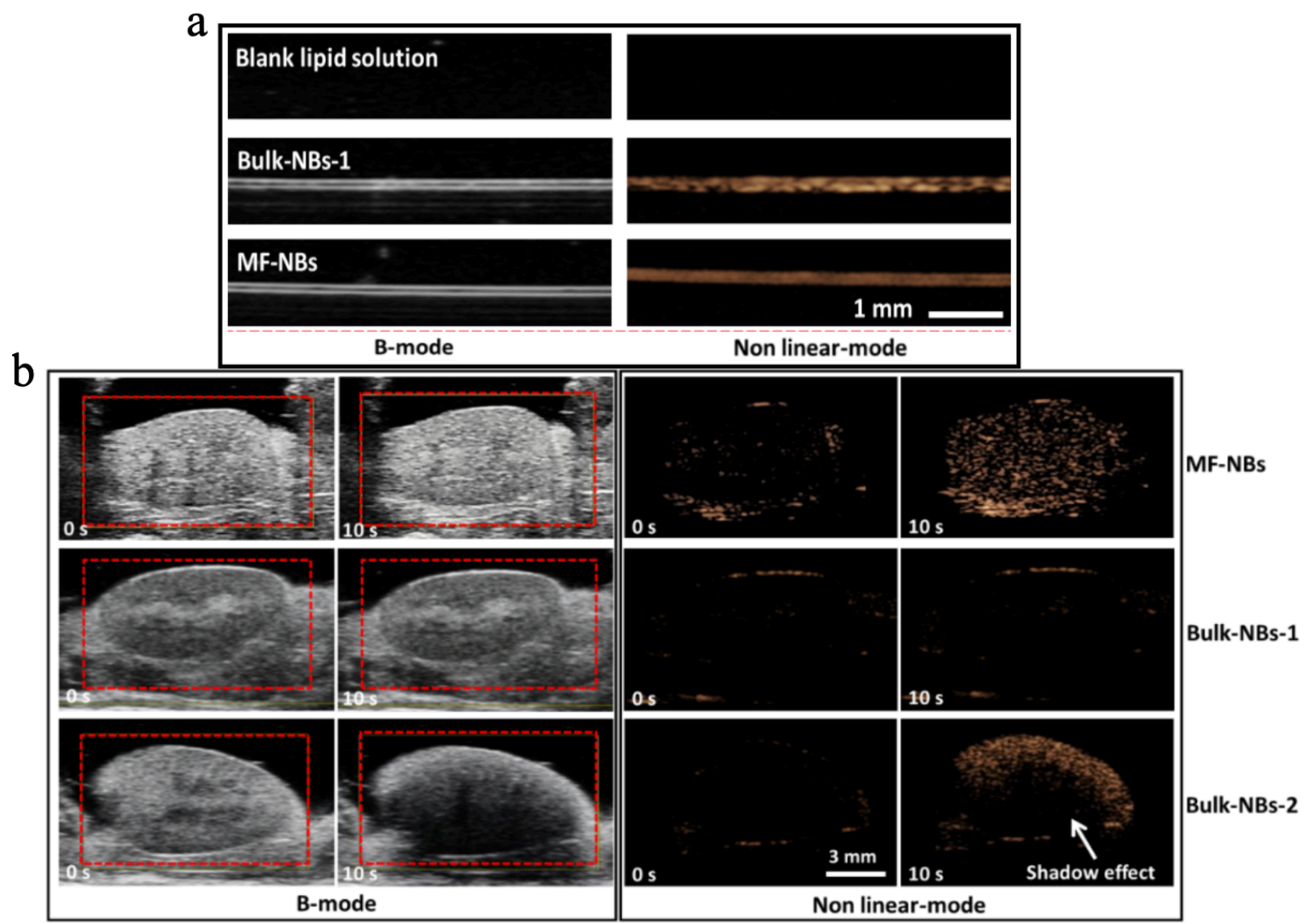

Figure 4.3 In vitro and in vivo contrast-enhanced ultrasound imaging with nanobubbles. Ultrasound signals are generated and collected by an $18 \mathrm{MHz}$ MS250 transducer (a) In vitro ultrasound images of nanobubbles under different contrast modes: B-mode (left), and non-linear mode (right), in a blood-vessel-like phantom. The nanobubbles are characterized by RMM: The MF-NBs have a diameter of $328 \mathrm{~nm} \pm 49 \mathrm{~nm}(\mathrm{n}=3)$, a concentration of $8.07 \times 10^{7} \mathrm{~mL}^{-1}$, and a polydispersity index of 0.148 ; The Bulk-NBs-1 have a diameter of $418 \mathrm{~nm} \pm 121 \mathrm{~nm}(\mathrm{n}=3)$, a concentration of $7.67 \times 10^{7} \mathrm{~mL}^{-1}$, and a poly-dispersity index of 0.288 . (b) In vivo images of nanobubbles in the kidney of a mouse captured in the brightness mode (i.e., B-mode) and nonlinear ultrasound mode. The white arrow points to the shadowing effect due to ultrasound attenuation by Bulk-NBs-2. The images are taken at $0 \mathrm{~s}$ and $10 \mathrm{~s}$ after the injection of the nanobubble solution. Bulk-NBs-1 are diluted 1,000 times from Bulk-NBs-2 to match the concentration of MF-NBs. MF-NBs are generated using the $0.16 \mathrm{wt} \% \mathrm{C}_{3} \mathrm{~F}_{8}$ in $\mathrm{N}_{2}$. Bulk-NBs-1 and Bulk-NBs-2 are generated using pure $\mathrm{C}_{3} \mathrm{~F}_{8}$ gas. Mouse kidneys in all experiments are directly exposed to the ultrasound transducer. The ultrasound imaging for contrast mode is at $4 \%$ power ( 2 $\mathrm{MPa})$, and for B-mode is at $1 \%$ power $(0.9 \mathrm{MPa})$. 
In the in vitro and in vivo experiments, we find that the MF-NBs may have unique advantages over conventionally made Bulk-NBs for ultrasound imaging. In the ultrasound imaging of an artificial blood vessel, the MF-NBs exhibit homogeneity of signal compared to Bulk-NBs (Figure 4.3a), with a comparable brightness. We attribute this improvement to the better monodispersity of MF-NBs, which cannot be realized with traditional bulk preparation methods. The monodisperse bubbles scatter ultrasound waves more uniformly, while poly-disperse Bulk-NBs scatter ultrasound less uniformly, likely due to the wider range of bubble sizes.

In in vivo imaging experiments of a mouse kidney, MF-NBs show a better ultrasonic contrast enhancement compared to the Bulk-NBs with a similar bubble concentration (Figure 4.3b). The brightness of the non-linear contrast mode images significantly increases after injection of $100 \mu \mathrm{L}$ of MF-NBs, while there is only a slight change in non-linear contrast mode image brightness after injection of the control group and Bulk-NBs-1 with approximately comparable size and bubble concentration in the MF-NBs. This enhancement is also reflected in B-mode images of MF-NBs and Bulk-NBs-1.

One plausible explanation of the strong echo activity of these MF-NBs may be firstly due to the enhanced non-linear scattering due to potential shell buckling of MF-NBs during the shrinkage. Secondly, for the mono-disperse MF-NBs, constructive interactions of the collective nonlinear behavior of interacting microbubble clusters may be more likely to happen compared to BulkNB. ${ }^{198}$ Our observations suggest that, in this series of imaging experiments, comparable contrast brightness can be achieved if bulk nanobubbles, such as Bulk-NBs-2, of a 1,000 times higher concentration, are used.

However, increasing nanobubble concentration with conventionally-made Bulk-NB solution may lead to more ultrasound attenuation ${ }^{10}$ due to larger nanobubbles in the poly-disperse bulk solution resulting in shallower penetration. In experiments with Bulk-NBs-2 (Figure 4.3b), we find decreased signal strength in the deeper sections of the kidney, as indicated in the figure by white arrows, corresponding to a larger distance from the ultrasound transducer. The decrease of ultrasound signal is in agreement with previous experimental observations, ${ }^{195}$ which find that ultrasound contrast enhancement increases with nanobubble concentration up to $10^{9} \mathrm{~mL}^{-1}$, but further increases of nanobubble concentration result in acoustic shadowing due to attenuation. We 
note that our method can yield nanobubble solutions with concentrations around $10^{8} \mathrm{~mL}^{-1}$, which should be suitable for contrast enhancement. ${ }^{195}$ This approach also has the potential to produce higher concentration nanobubbles with modifications to the device geometry and flow conditions. In clinical situations, different tumors may have various depths or vascularity that could lead to a choice of nanobubble concentration based on imaging trade-offs (a target echogenicity at different imaging depths). The results demonstrate advantages of the mono-disperse nanobubbles generated by our microfluidics method, which may find applications in diagnostic ultrasound imaging, therapeutic ultrasound and in-situ micro- or nano-surgery.

\subsection{Conclusion}

In summary, we describe for the first time, size-tunable and mono-disperse nanobubbles generated using a simple microfluidic approach. By controlling the fraction of $\mathrm{C}_{3} \mathrm{~F}_{8}$ and $\mathrm{N}_{2}$ gas in the bubble and tuning the initial size of bubbles generated at a flow-focusing microfluidic geometry, we are able to control the degree of shrinkage of microbubbles to yield nanobubbles of target sizes. In both in vitro and in vivo ultrasound imaging experiments, these MF-NBs show excellent signal homogeneity and contrast.

Compared to slightly modified conventional generation protocols of bulk nanobubbles, our method improves controllability and uniformity of nanobubbles, and retains a tunable concentration (up to $10^{8} \mathrm{~mL}^{-1}$ ) of bubbles. This method may be extended to produce nanobubbles of other gas core compositions, and may be applicable for various fields from environmental science to biomedical engineering. ${ }^{199}$

\subsection{Methods}

\subsubsection{Microfluidic Device Fabrication and Operation}

We used standard photolithography and soft lithography to make the microfluidic device consisting of a cross junction and a serpentine channel with a $50 \mu \mathrm{m}$ height. We supplied the gas mixture using Tygon tubing (Saint-Gobain S.A.) and a syringe pump (Harvard Instruments). To 
obtain bubbles with the desired initial size, we controlled the injection of the lab-made gas mixture (see Note C.1) using a syringe pump, while the industrial gas mixture (SynQuest) was pumped using a pressure regulator. The aqueous phase was supplied at $1 \mathrm{~mL} \mathrm{hr}^{-1}$ using a syringe pump. The shrunken nanobubbles were collected from the device outlet in a $3 \mathrm{~mL}$ glass vial, and then stored inside a fridge at $4{ }^{\circ} \mathrm{C}$ before TEM and RMM characterizations.

\subsubsection{Preparation of the Lipid Solution}

A mixture of the following lipids was first prepared in a glass vial:

a. DPPC (1,2-dipalmitoyl-sn-glycero-3-phosphocholine) (Avanti Polar Lipids Inc.): $60.1 \mathrm{mg}$

b. DPPA (1,2-dipalmitoyl-sn-glycero-3-phosphate (sodium salt)) (Avanti Polar Lipids Inc.): $10 \mathrm{mg}$

c. DPPE (1,2-dipalmitoyl-sn-glycero-3-phosphoethanolamine) (CordenPharma): $20 \mathrm{mg}$

d. DSPE-mPEG (2000) (1,2-distearoyl-sn-glycero-3-phosphoethanolamine-N[carboxy(polyethylene glycol)-2000] (sodium salt)) (Avanti Polar Lipids Inc.): $10 \mathrm{mg}$

Then, we added $15 \mathrm{~mL}$ of Dichloromethane (MilliporeSigma) to thoroughly dissolve the lipids and gently evaporate them overnight to remove all solvents. The amorphous lipids formed a thin white film on the wall. The aqueous solution was prepared by mixing the following chemicals:

a. PBS (phosphate-buffered saline) solution ( $\mathrm{pH}$ 7.4) (Thermo Fisher Scientific Inc.): $90 \mathrm{~mL}$

b. Glycerol (MilliporeSigma): $5 \mathrm{~mL}$

c. Pluronic F-68 (Thermo Fisher Scientific Inc.): $2.5 \mathrm{~mL}$

d. Propylene glycol (MilliporeSigma): $2.5 \mathrm{~mL}$ 
Next, we dissolved the lipid mixture in the aqueous solution and sonicated them in a $50{ }^{\circ} \mathrm{C}$ water bath for 2 hours. A filtration membrane (Acrodisc Syringe Filters, $3 \mu \mathrm{m}$ pore size, VWR) was then used to remove undissolved lipids and oversized aggregates from the solution.

\subsubsection{Preparation of Bulk Nanobubbles}

We transferred $3 \mathrm{~mL}$ of the hydrated lipid solution into a $5 \mathrm{~mL}$ glass vial, which was then sealed with a rubber cap. We removed air of the vial by applying a vacuum with a $20 \mathrm{~mL}$ syringe connected to a $25 \mathrm{G}$ needle. The vacuum process was repeated 3 times. Octafluoropropane $\left(\mathrm{C}_{3} \mathrm{~F}_{8}\right)$ was filled through a $25 \mathrm{G}$ needle from a $10 \mathrm{~mL}$ syringe prefilled with $\mathrm{C}_{3} \mathrm{~F}_{8}$ gas, until the pressure was equalized. The glass vial containing the lipid solution and $\mathrm{C}_{3} \mathrm{~F}_{8}$ was then shaken vigorously for 45 seconds using a mechanical shaker (VialMix, Bristol Myers Squibb) to generate a milky homogeneous mixture of microbubbles and nanobubbles. This mixture was inverted with the rubber cap facing down and centrifuged at $500 \mathrm{rpm}$ for 2 minutes. We used a $1 \mathrm{~mL}$ syringe and a $21 \mathrm{G}$ needle to gently remove $1 \mathrm{~mL}$ of the nanobubbles.

\subsubsection{RMM Measurements}

We took a $1 \mathrm{~mL}$ of the nanobubble solution in a microcentrifuge tube $(2 \mathrm{~mL}$, Eppendorf Canada) and their particle sizes and buoyant mass were measured using RMM (Archimedes, Malvern Instruments). In our experiments, we utilized a nanosensor that provides measurement in the range spanning from $100 \mathrm{~nm}$ to $2 \mu \mathrm{m}$. Sensors were calibrated using NIST traceable $500 \mathrm{~nm}$ polystyrene beads (ThermoFisher 4010S) in water solution. Sensor calibration was finalized after 100 particles were detected. Bulk samples after centrifugation were diluted 1000 times in PBS. This diluted concentration resulted in an acceptable limit of detection (i.e., $0.01 \mathrm{~Hz}$ ) and coincidence $(<10 \%)$. Samples were loaded into the system for 90 seconds at 10 psi and analyzed at a low pressure (i.e., 5 psi). Data was exported from the Archimedes software and analyzed for positive and negative counts. A density of $0.08 \mathrm{gr} \mathrm{mL}^{-1}$ for positively buoyant particles (i.e., nanobubbles) and $1.3 \mathrm{gr} \mathrm{mL}^{-1}$ for negatively buoyant particles (i.e., lipid aggregates) were inputted into the software to convert the measured mass to a particle diameter. ${ }^{195,200}$ The sharp cut-off in size observed in Figure 4.2d for the buoyant nanobubbles was a result of the limit of detection of 
the sensor used in our measurements. This limit of detection was determined at the start of each measurement and accounts for the baseline noise.

\subsubsection{TEM Measurements}

Carbon coated TEM grid was first cleaned by a plasma cleaner. 2\% uranyl acetate in distilled water was used for negative staining. One drop $(\sim 20 \mu \mathrm{L})$ of the nanobubble solution and three drops of DI water are pipetted on a $30 \mathrm{~cm}$ long parafilm. The grid was gently put to contact the top of NB drop for $2 \mathrm{~min}$. The grid was then transferred to contact with the DI water drops for dilution. Each dilution step was lasted for $1 \mathrm{~min}$. When the sample was fully diluted, $20 \mu \mathrm{L}$ of stain was applied on the grid. Negative staining was done for $1 \mathrm{~min}$ in dark. The excess stain was then blotted by a filter paper and the grid was dried before imaging.

\subsubsection{In Vitro Ultrasound Imaging}

Two groups of nanobubbles are prepared for characterizing their in vitro echogenicity: MFNBs was collected from the microfluidic shrinkage platform. Bulk-NBs-2 was diluted 1000 times in PBS to make Bulk-NBs-1, which has an almost equal bubble concentration to that of MF-NBs. Bubble solutions were placed inside a gelatin $(10 \% \mathrm{w} / \mathrm{v})$ channel with a length of $50 \mathrm{~mm}$ and a diameter of $0.25 \mathrm{~mm}$. A pre-clinical ultrasound scanner (VisualSonics 2100) was then used in the nonlinear contrast harmonic imaging mode at a harmonic frequency of $18 \mathrm{MHz}$, dynamic range of $35 \mathrm{~dB}$, and a gain of $40 \mathrm{~dB}$.

\subsubsection{In Vivo Ultrasound Imaging}

Animal experiments were conducted in compliance with the St. Michael's Hospital Animal Ethics Committee and conformed to the Canadian Council on Animal Care guidelines. In all procedures, the mice (16 weeks old, CD1 background) were anesthetized with 3\% isoflurane with $1 \mathrm{~L} \mathrm{~min}^{-1}$ oxygen. The skin of mice belly was removed carefully to make the kidney exposed to the transducer directly, with the protection of imaging gel. In each experiment, we injected a 100 $\mu \mathrm{L}$ of the bubble solution through the tail vein. Contrast-enhanced ultrasound images were acquired with a Vevo 2100 (VisualSonics) at $1 \mathrm{fps}$ and $18 \mathrm{MHz}$. 


\section{Acoustic Microflows Generated by Oscillating Cells}

The work presented in this chapter is based on the following article, ${ }^{201}$ which is published in a peer-reviewed journal Small, and is reproduced here with permission from John Wiley and Sons.

Salari, A., Appak-Baskoy, S., Ezzo, M., Hinz, B., Kolios, M.C., and Tsai, S.S.H. Dancing with the cells: Acoustic microflows generated by oscillating cells. Small 1903788, 1-7 (2019).

\section{Author's Contribution}

For the article presented here, the author wrote the manuscript and designed, fabricated, and tested the devices. Other than the atomic force microscopy measurements, the author designed and conducted all the experiments and collected and analyzed data. S. Appak-Baskoy helped with providing chemicals, staining protocols, discussions, and comments on the manuscript. M. Ezzo and B. Hinz conducted and analyzed atomic force microscopy measurements.

\subsection{Introduction}

Acoustofluidics, the field of microscale fluid manipulation using acoustics, has utility in many applications, including drug delivery, ${ }^{48,202}$ microorganism manipulation, ${ }^{88,203,204}$ analyte mixing, ${ }^{205-208}$ and lab-on-a-chip fluid pumping. ${ }^{209,210}$ In a typical acoustofluidic system, either Rayleigh surface acoustic waves (SAW) or bulk waves traveling on the surface or in the bulk of a 
microfluidic device, respectively, is the dominant wave propagation mode. Such an acoustic actuation mechanism can force the microchannel walls, ${ }^{203,204,211}$ microbubbles, ${ }^{88,206,208}$ or sharpedge elastic geometries ${ }^{86,205,212}$ embedded inside the microfluidic device to oscillate. A result of this oscillation is a non-periodic fluid motion generated near the oscillating object in its surrounding liquid. This fluid flow is called acoustic microstreaming, which is a non-linear phenomenon, and is directly related to the second order acoustic field. ${ }^{213,214}$

Acoustic streaming flows are also possible in biological systems. One example is streaming flows generated in the mammalian inner ear upon a perturbation caused by an incident sound wave. ${ }^{215-217}$ Similarly, the self-propulsion mechanism of some flagella-less microorganisms, such as the cyanobacterium Synechococcus, is hypothesized to be acoustic streaming. ${ }^{218,219}$ This hypothesis is also inspiring the development of autonomous microswimmers propelled by acoustic streaming. ${ }^{220-222}$ Other biological systems, such as cells, have viscoelastic properties similar to many materials, such as microbubbles, that produce acoustic microstreaming under acoustic excitation. Despite this similarity, cell-induced microstreaming has not been observed or reported for any biological species in vitro.

Here, we report for the first time, microstreaming flow patterns generated by adherent cells. In our platform, we generate acoustic waves in order to force the adherent cells to oscillate inside a microfluidic channel. We monitor flow tracers surrounding single-cells, to investigate cellinduced microstreaming when the structure and environment of the cells are altered. We modulate the volume and physical properties of cells by changing the extracellular osmotic pressure. We also conduct an enzymatic treatment using trypsin and two chemical treatments using Paraformaldehyde and Cytochalasin D. Our observations reveal that the maximum streaming velocity generated by a cell is correlated with the cell stiffness. Therefore, cell-induced microstreaming parameters, such as flow pattern and velocity magnitude, may be measured as label-free proxies for cell mechanical properties. 


\subsection{Working Principle}

Figure 5.1a shows a schematic diagram of the fabricated device consisting of a thin-walled polydimethylsiloxane (PDMS) microchannel attached onto a glass substrate next to a piezoelectric (PZT) transducer. Once the transducer is actuated, acoustic waves are created and propagate through the substrate. In thin substrates, where the thickness $d$ is on the order of, or smaller than, the sound wavelength, the wave propagation is mainly via Lamb waves. ${ }^{71}$ Lamb waves are known to independently propagate in symmetric and antisymmetric modes. As opposed to Rayleigh waves, the speed of sound in Lamb waves is a function of frequency. When the product of substrate thickness and sound frequency, i.e., $f d$, is relatively low (in our study, the product $f d=0.5-10 \mathrm{MHz}$ $\mu \mathrm{m}$ ) only one symmetric mode is dominant. In a typical acoustofluidic device, sound waves reflected from boundaries form a pseudo-standing wave in the microfluidic channel. ${ }^{211}$ At certain frequencies and substrate thicknesses, e.g., $f \approx 85 \mathrm{kHz}$ and $d \approx 170 \mu \mathrm{m}$, the actuated PZT transducer can cause transverse substrate oscillations that give rise to a slow microstreaming flow in the microfluidic channel ${ }^{86}$ Here, we choose to attach adherent cells onto the substrate, which results in microstreaming flow (see Video D.1) that is an order-of-magnitude stronger than the ones generated due to the oscillations of other surrounding objects (i.e., channel wall or substrate; see Video D.2). The cell-induced microstreaming flow is studied in a $500 \mu \mathrm{m} \times 500 \mu \mathrm{m}$ region of interest (ROI) at the center of the microfluidic channel that is $\sim 4 \mathrm{~mm}$ away from the PZT disk, at an acoustic anti-nodal location, where a strong microstreaming flow is observed. 


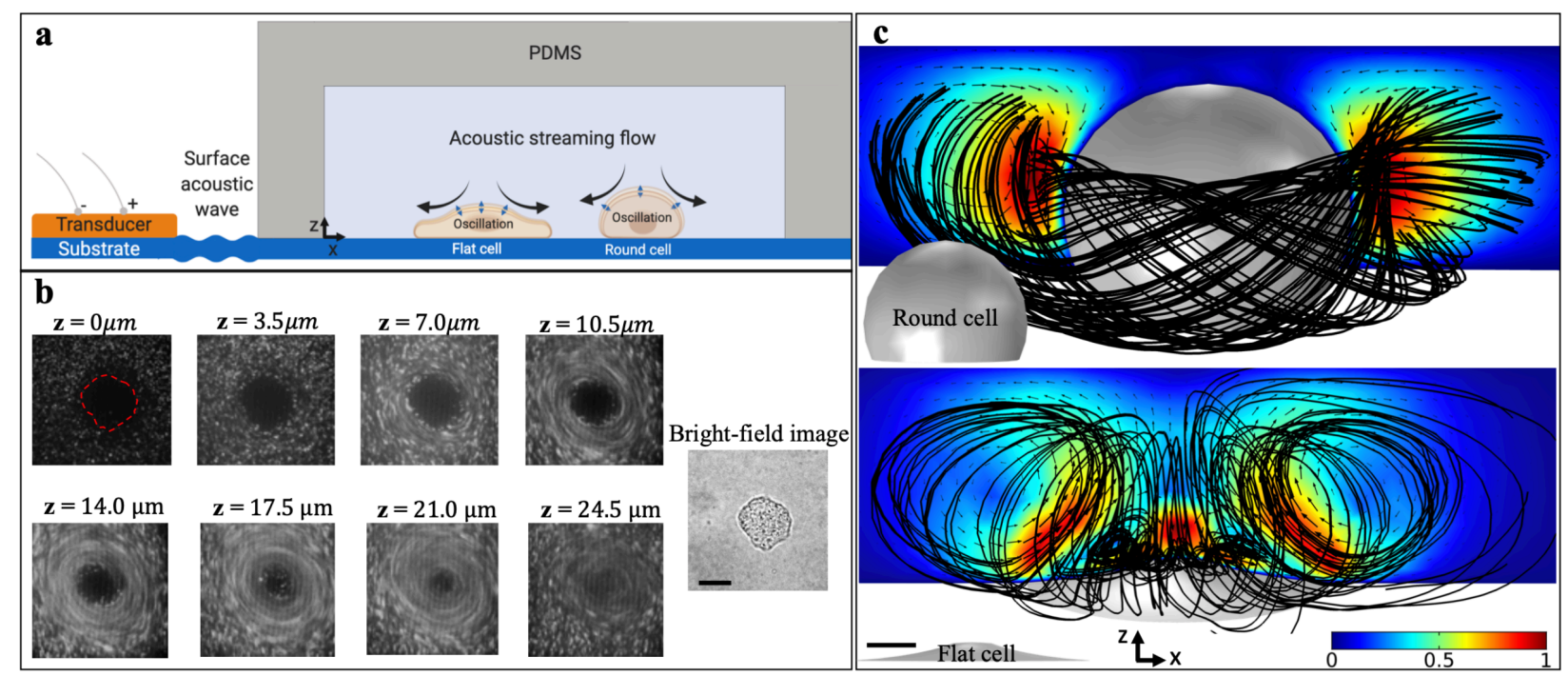

Figure 5.1 (a) Schematic diagram (not to scale) of cell oscillation driven by acoustic waves. A thin-walled PDMS channel contains the cells. The acoustic waves are generated by a PZT disk attached onto the substrate next to the channel. Acoustic streaming flow is generated as a result of cell membrane oscillations. (b) Confocal images of fluorescent particle tracers taken at different heights from the substrate surface in a channel with an overall height of $25 \mu \mathrm{m}$. The MCF-7 cell is shown in the bright-field view and is outlined by the red dashed line. As depicted, a circular flow is generated in the liquid bulk above the cell, which has its maximum velocity near the channel mid height at $z \approx 14-21 \mu \mathrm{m}$. The streaming velocity approaches zero near the top and bottom surfaces of the channel due to no-slip boundary conditions. (c) The streaming flow is also simulated numerically for two model cells: a round and a flat cell. As illustrated, helical-shape streamlines are induced surrounding each cell. The round cell's helical streamlines have a higher pitch than the streamlines of the flat cell. Regions of maximum streaming flow are located close to the cell membrane. The color legend shows the non-dimensionalized velocity magnitude, and the arrows depict the $2 \mathrm{D}$ velocity field on a cross-section of the flow. The scale bars represent 10 $\mu \mathrm{m}$. The schematic in (a) is created with Biorender.com.

Since the wavelength of the acoustic field in our device is on the order of centimeters, which is much larger than the area of the ROI, the variation of acoustic amplitude within the ROI is negligible (i.e., less than $1 \%$ ). We fabricate a relatively wide (i.e., $1 \mathrm{~mm}$ width) microfluidic channel so that the microstreaming caused by PDMS wall oscillations does not affect the observations made in the ROI, which is located in the middle of the channel. Once the channel 
surface is coated with fibronectin, we inject a dilute cell suspension into the channel. When singlecells are fully adhered, we expose the cells to acoustic waves by actuating the PZT disk (Figure 5.1a) at a certain frequency and amplitude.

Figure $5.1 \mathrm{~b}$ shows snapshots of flow patterns formed by fluorescent tracer beads when a single MCF- 7 breast cancer cell is driven at $f=98 \mathrm{kHz}$. The cell is adhered onto the substrate in a $25 \mu \mathrm{m}$ height channel. Confocal images taken at different heights inside the channel show a circular flow pattern surrounding the cell. The flow velocity has its minimum near the top and bottom channel surfaces and reaches a maximum at around $z \approx 14-21 \mu \mathrm{m}$ (see Videos D.3D.5). Furthermore, we simulate this fluid flow numerically using perturbation theory (see Note D.1), assuming that the apical membrane of the cell oscillates equally in all three directions. As depicted in Figure 5.1c, the morphology of the cell can impact the circular flow pattern. For instance, for a cell with a semi-spherical round shape, a low-pitch helical flow forms surrounding the cell, whereas, for a flat cell, a high-pitch helical flow is generated.

\subsection{Physical Aspects}

In this section, we present the results of monitoring two MDA-MB-231 breast cancer cells while the actuation frequency $f$ is swept from $10 \mathrm{kHz}$ to $100 \mathrm{kHz}$, to investigate frequencydependant cell-induced microstreaming flow. Figure 5.2a-c show the traces of $1 \mu \mathrm{m}$ polystyrene beads when they are dragged into the flow due to the cells being excited at different frequencies. 

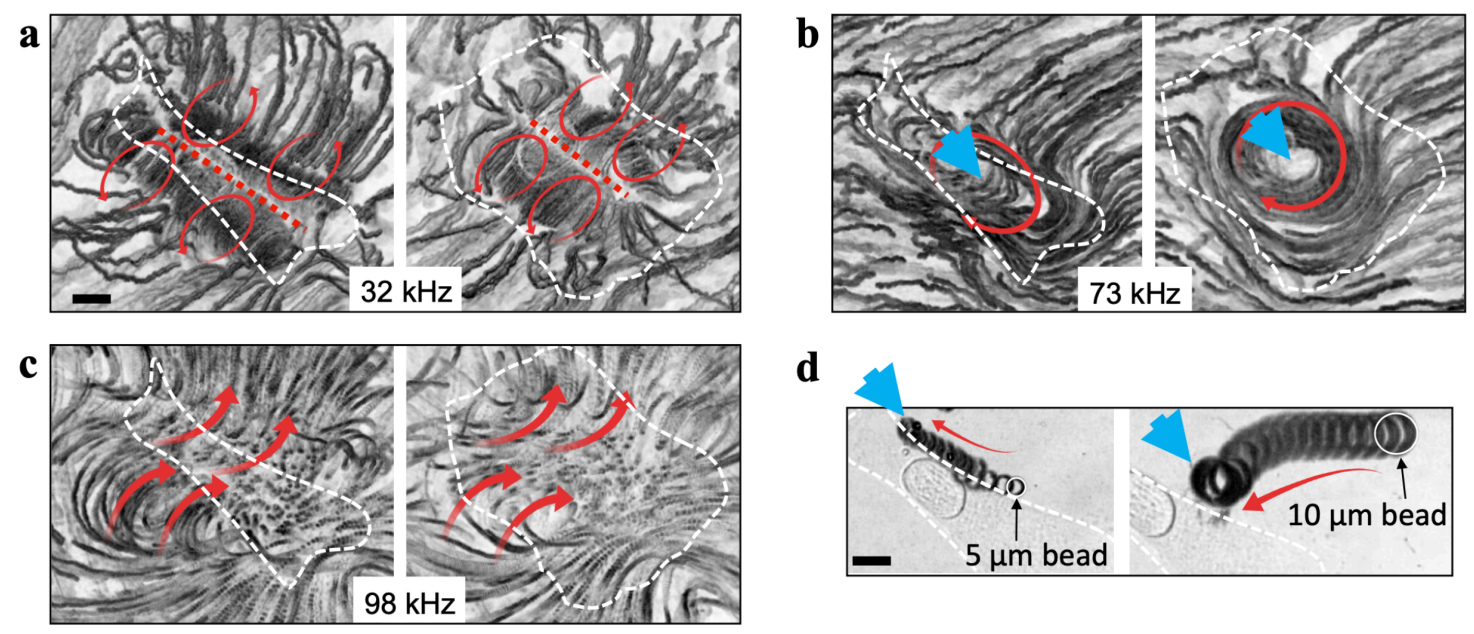

d

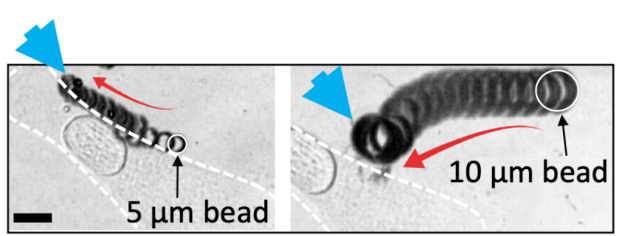

Figure 5.2 (a-c) Different streaming flow regimes generated surrounding two MDA-MB-231 cells at different actuation frequencies. $1 \mu \mathrm{m}$ polystyrene beads are used as flow tracers. (a) Two almost symmetric sets of out-of-plane vortices are generated on two sides of each cell when excited at $32 \mathrm{kHz}$, as opposed to (b) planar vortices which form over the entire cell surface at $73 \mathrm{kHz}$. (c) The flow regime changes to a combination of in- and out-of-plane motion at higher frequencies (e.g., $\sim 98 \mathrm{kHz}$ ). (d) As the bead diameter increases, the acoustic radiation force becomes the dominant force acting on the bead, causing (d-left panel) a $5 \mu \mathrm{m}$ and (d-right panel) a $10 \mu \mathrm{m}$ polystyrene bead to partially follow the flow streamlines and eventually remain stationary on the boundary between the cell and substrate. The blue arrows in (d) show the final location of the beads. White dashed lines show the cells' outlines. The scale bars represent $10 \mu \mathrm{m}$.

At $f=\sim 32 \mathrm{kHz}$, two distinct sets of counter-rotating out-of-plane vortices form above the cell (see Video D.6). In Figure 5.2a, red dashed lines separate the two regions of these microvortices sets that form along the long axis of the cell (cell outlined in white dashed lines). Our observations indicate that at this frequency, each set of vortices tend to form over an area along the cell's longitudinal axis for an asymmetric cell. At $f=\sim 73 \mathrm{kHz}$ (Figure 5.2b), however, only one set of planar vortices is generated with the center of rotation being near the maximum height of the cell (marked by blue arrows in Figure 5.2b and Figure D.1) (see Video D.7).

At even higher frequencies, e.g. $\sim 98 \mathrm{kHz}$, the streaming flow turns into a combination of planar and out-of-plane flows (Figure 5.2c). At this frequency, cells exhibit their highest microstreaming velocity (see Video D.8), and therefore, we choose this actuation frequency for 
the subsequent treatment experiments to better visualize the effect of different treatments on the velocity magnitude. As adhered cells can form various shapes and geometries, the frequencydependant pattern discussed above can vary across cells with different shapes. We hypothesize that different oscillation modes of the cell membrane may be responsible for the observation of various frequency-dependant flow regimes. This is analogous to the various frequency-dependant flow patterns generated by an acoustically driven microbubble. ${ }^{88}$

When beads with larger sizes (e.g., $d_{p}=10 \mu \mathrm{m}$ ) are introduced into our device we find that they partially follow the streaming flow and, in some cases, are eventually trapped at a location on the boundary between the cell and substrate (indicated by blue arrows in Figure 5.2d) (see Videos D.9 and D.10). This observation can be due to the size-dependant forces acting on the beads. Typically, a bead with a diameter $d_{p}$ flowing in an acoustofluidic device experiences two forces arising from the flow hydrodynamics and the acoustic field. ${ }^{88}$ The drag force $F_{D}$, caused by the microstreaming flow, is directly proportional to the bead diameter, i.e., $F_{D} \propto d_{p}$, while the acoustic radiation force $F_{R}$, due to the scattering of the incident waves from the oscillating cell, is related to the bead diameter as $F_{R} \propto d_{p}^{3} .{ }^{88,223}$ These relationships imply that the drag force is the dominant force for a smaller bead (e.g., $d_{p}=1 \mu \mathrm{m}$ ). In this regime, the bead acts as a flow tracer, as shown in Figure 5.2a-c. However, as the size of the beads increase (e.g., $\left.d_{p}=10 \mu \mathrm{m}\right)$, the radiation force becomes the dominant force causing the beads in Figure $5.2 \mathrm{~d}$ to be attracted towards the cell.

In the following experiments, we identify some of the governing parameters that can alter the cell oscillation behaviour, and thus, affect the magnitude of streaming velocity. We add flow tracers (i.e., $1 \mu \mathrm{m}$ beads) to every solution before it is introduced into the microfluidic channel, to help with flow visualization. Then, the average velocity of at least 10 beads with the highest speed surrounding each single-cell is measured using a semi-automated protocol, ${ }^{224}$ to determine the maximum microstreaming velocity (see Note D.2 for more measurement details). The results of three trials (i.e., three different groups of cells at the ROI) of microstreaming measurements in the ROI demonstrating the repeatability of the measurements are shown in Figure D.2.

As shown in Figure 5.3a, the thickness of the substrate has a significant impact on the resultant streaming flow. Cells adhered onto a glass substrate with a thickness of $100 \mu \mathrm{m}$ generate 
the highest fluid velocity, and as the substrate thickness increases, the streaming flow becomes weaker. This result suggests that the dominant acoustic wave propagation mechanism in our device is in the form of Lamb waves, since propagation characteristics of Lamb waves are related to substrate thickness. ${ }^{71}$ We validate this hypothesis by changing the PZT configuration such that the transducer and the microfluidic channel are attached onto the opposite surfaces of the substrate. We find that the cells in a device with this new configuration can also induce microstreaming (see Figure D.3). This suggests that both surfaces of the substrate contribute to the propagation of sound waves - this is a feature of Lamb waves. ${ }^{71,225}$

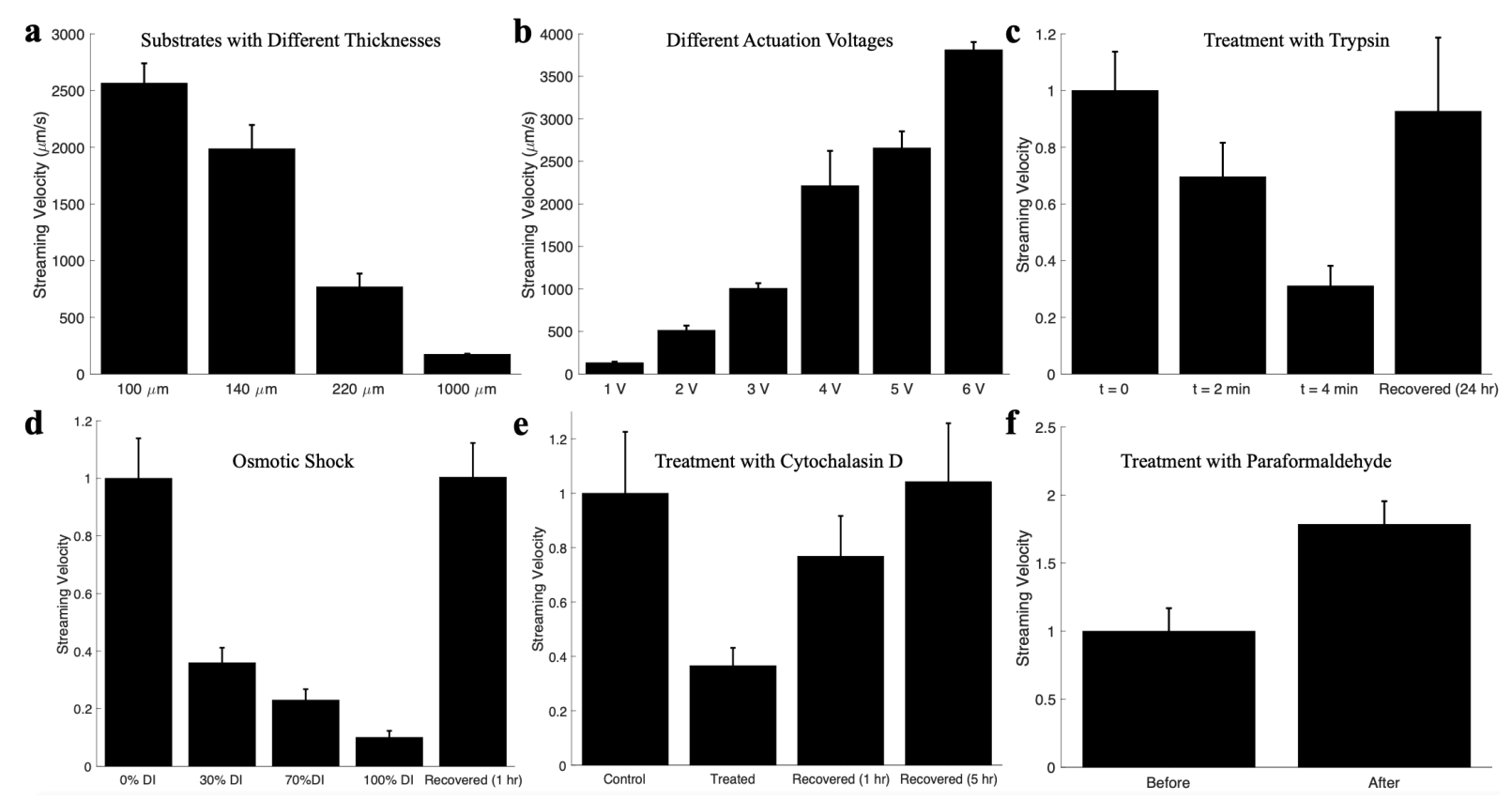

Figure 5.3 The maximum streaming velocity induced by MDA-MB-231 cells under different conditions. (a) Substrates with different thicknesses in the range of $100 \mu \mathrm{m}$ to $1 \mathrm{~mm}$. Increasing the substrate thickness decreases the streaming velocity. (b) Different actuation voltages in the range of 1 to $6 \mathrm{~V}$ (rms). A higher actuation voltage corresponds to a higher oscillation amplitude of the substrate, which is coupled to the cell, causing larger amplitude cell oscillations and a stronger microstreaming effect. (c) Trypsinization over $4 \mathrm{~min}$. As the adhesion proteins are dissociated and the projected area of the cells decreases, the cell attachment to the substrate is weakened, causing the streaming velocity to decrease. The streaming flow, however, is recovered within $24 \mathrm{hr}$ post-treatment. (d) Osmotic pressure variations over $10 \mathrm{~min}$. Cell exposure to hypotonic solution results in an increase in the cell volume and changes in cell physical properties, 
which in turn results in a decrease in the streaming velocity. The streaming flow can be recovered within $1 \mathrm{hr}$ post-treatment. (e) Treatment with $4 \mu \mathrm{M}$ Cytochalasin D. When F-actin proteins are disrupted, the cell loses its stiffness, and consequently, the streaming velocity decreases. This change in streaming flow is recovered within $5 \mathrm{hr}$ post-treatment. (f) Fixation with $4 \%$ Paraformaldehyde. The cross-linked structure formed upon fixation causes the entire cell to oscillate as a uniform meshwork which yields a stronger streaming flow. The error bars represent one standard deviation $(\mathrm{n} \geq 10, \mathrm{p} \leq 0.05)$.

We also investigate the effect of the amplitude of cell oscillation on the magnitude of streaming velocity, by sweeping the PZT actuation voltage from 1 to $6 \mathrm{~V}$ (rms) (Figure 5.3b). Similar to the microstreaming caused by the oscillation of bubbles or elastic materials, ${ }^{88}$ the microstreaming velocity induced by cells tends to increase with the PZT actuation voltage, and thereby, the oscillation amplitude of the cell membrane.

We keep the substrate thickness at $100 \mu \mathrm{m}$ and the actuation voltage at $5 \mathrm{~V}(\mathrm{rms})$ constant for the rest of our experiments, to maintain a strong microstreaming effect that improves our measurement precision. Microstreaming velocities are normalized by the velocity of the control experiment (i.e., before treatment) to facilitate better comparisons between the effects of each treatment in the next section.

\subsection{Biological Aspects}

In our setup, microstreaming induced by floating cells and particles are very weak, as the only way cells can be excited is through the waves leaked into the microfluidic channel. ${ }^{211} \mathrm{We}$ find that the cell attachment to the substrate is critical for the formation of cell-induced microstreaming. Hypothetically, disruption of adhesion proteins should therefore weaken the cell oscillation, as the attachment of adherent cells to the glass substrate is maintained through these proteins. We examine this hypothesis by replacing the culture medium with $0.25 \%$ Trypsin-EDTA and monitoring the streaming flow for $4 \mathrm{~min}$. We limit the experiments to $4 \mathrm{~min}$ because longer exposure times can cause detachment of the cells from the substrate, and also permanently damage the cells surface proteins, which can eventually cause cell death. ${ }^{226}$ 
Figure $5.3 \mathrm{c}$ shows a significant decrease in the streaming velocity after the cells are exposed to Trypsin for $4 \mathrm{~min}$. This observation can be explained considering the following two effects. First, adhesion proteins at the focal points, which couple the acoustic wave with the cell, begin to dissociate. This weakens the cell's attachment to the substrate and the mechanical energy transfer from the substrate to the cell. Second, as a result of the first effect and as shown in Figure D.4, the projected area covered by the cell decreases. ${ }^{227-229}$ After trypsin is replaced with culture medium, the cells regain their adhesion proteins gradually, and the microstreaming effect is recovered over a 24-hr period.

In addition to trypsinization, the volume of a cell can also be manipulated by osmosis. Imposing various levels of osmotic pressure on the cells is achieved by replacing the culture medium with a mixture of the culture medium and deionized (DI) water at different ratios. Starting with an isotonic solution (i.e., 0\% DI), we inject three hypotonic solutions of 30\% (v/v) DI, 70\% DI and 100\% DI, successively. The entire 4-step solution change is conducted in 10 min with 2 min intervals for streaming measurements. A significant decrease in streaming velocity is observed immediately after the injection of $30 \%$ DI solution (Figure $5.3 \mathrm{~d}$ ). This could be due to the physical changes imposed to the cell, including the increase in volume ${ }^{228}$ (as shown in Figure D.5) and the decrease in actin filament polymerization, ${ }^{230}$ which may weaken the attachment of cell membrane to cytoskeleton. ${ }^{231}$ As a result, the propagation of acoustic energy from the substrate to the cell membrane can be disrupted. Once the osmotic shock is removed, the cell-induced streaming velocity recovers relatively fast (within $1 \mathrm{hr}$ ). Interestingly, we find that, cells exposed to a small hypotonic shock can exhibit inner streaming flows such that the cell membrane oscillation induces a microstreaming flow within the cell cytoplasm, revealed through the motion of intracellular vesicles (see Video D.11).

As a major contributor to the cell morphology, actin filaments play a critical role in maintaining the structural integrity of the cells. We, therefore, choose to target these filaments by incubating the cells with $4 \mu \mathrm{M}$ Cytochalasin D (CD) in culture medium (see Figure D.6). Figure 5.3e shows a significant decrease in the streaming velocity when the actin filaments of the cells are disrupted. When the drug solution is washed out, the polymerization of actin filaments begins so that the actin stress fibers constituting to the cytoskeletal structure are recovered. This is 
reflected in the streaming flow with a gradual increase in the velocity, which takes $\geq 5 \mathrm{hr}$ for the streaming effect to be recovered. This finding supports our hypothesis that a uniformly distributed cell cytoskeleton, especially actin filaments, contribute to a great extent to the magnitude of streaming flow. It should be noted that, we also target the cell microtubules using Nocodazole, however, our results (not shown here) show no significant reduction in streaming flow. These observations highlight that the microstreaming effect seems to be dependent on the changes happening to the cortical, and not the cytoplasmic, structure of the cell.

Finally, we investigate the effect of fixation on the cell-induced streaming flow using $4 \%$ Paraformaldehyde (PFA) which causes the cell proteins to become covalently cross-linked such that the entire cell converts into a meshwork. ${ }^{232}$ As depicted in Figure 5.3f, the PFA treatment nearly doubles the streaming velocity. This increase in streaming velocity may be due to the fixation-induced cytoskeletal changes, as the surface area and volume of cells are not significantly affected by PFA fixation. ${ }^{232}$ Based on simple mechanical modeling, a cell can be assumed as a combination of an elastic membrane (i.e., spring) enclosing a viscous cytoplasm (i.e., dashpot). ${ }^{233}$ In such modeling, the transition from a viscoelastic material to a covalently cross-linked meshwork, formed upon PFA treatment, can be regarded as a transition towards a more uniform elastic material with lower viscous properties. ${ }^{234}$ Therefore, a higher streaming velocity upon fixation can be explained by changes in cell viscoelastic properties.

It is reported that hypotonic solution ${ }^{235}$ and actin filament disruption ${ }^{236}$ can decrease the effective stiffness of the cell, while the formation of a meshwork structure caused by PFA treatment ${ }^{237}$ can enhance the cell stiffness. Here, we observe a similar trend of variation in the streaming velocity caused by these treatments, so there appears to be a strong correlation between cell stiffness and cell-induced microstreaming flow.

Young's elastic modulus is commonly used for specifying a cell's time-independent mechanical properties. ${ }^{238}$ To directly correlate the maximum streaming velocity to the Young's modulus of cells under the same experimental conditions, we use atomic force microscopy (AFM) ${ }^{239}$ The mean Young's moduli obtained from AFM indentation measurements for cells treated with CD and PFA are shown in Figure D.7. Live cells exhibit an average Young's modulus of $1.38 \mathrm{kPa}$. Once the cells are treated with $\mathrm{CD}$, actin filaments are disrupted ${ }^{240}$ which results in 
three-fold lower average Young's modulus of $0.48 \mathrm{kPa}$. Cells treated with PFA, however, are stiffer than live cells with $\sim$ five-fold higher average Young's modulus of $6.57 \mathrm{kPa}$ compared with controls.

By comparing the results shown in Figure 5.3e-f and Figure D.7, we thus find that the maximum streaming velocity in fact increases with increasing cell stiffness and decreases with decreasing cell stiffness. To further investigate the robustness of this relationship, stiffness measurements of different cell lines are required which can be investigated in the future. Similar to the elasticity of cells that can span over a wide range even for the same cell type, ${ }^{239}$ the magnitude of streaming velocity induced by single-cells can also vary depending on various factors including the degree of cell adhesion to the substrate, cell cycle, and ambient temperature. Figure D.8 shows the variation in streaming velocity for individual cells before and after treatment with PFA. These results show that, while the streaming velocity varies between different cells, streaming velocity is overall higher after treatment.

\subsection{Conclusion}

We report for the first time that biological cells, similar to microbubbles and other elastic materials, are able to induce acoustic microstreaming flow, when they are exposed to an acoustic field. The acoustofluidic strategy presented here allows us to expose adherent cells to controlled acoustic waves in a precise and scalable platform such that oscillations of the cell induce microstreaming flow in the bulk of its surrounding liquid. While our experiments are on a singlecell basis, a cell monolayer can also be studied in a single field of view, with simultaneous measurements on a larger population of cells.

Our results suggest that the cell morphology and the acoustic actuation parameters can influence the streaming flow pattern and magnitude. The cell-induced microstreaming can also drag a floating bead into motion or attract it towards the cell membrane depending on the bead size. By treating the cells with different cytoskeletal drugs, we verify that when cells have a higher concentration of actin filaments (i.e., stress fibers), larger projected area, and lower volume, the induced microstreaming flows are stronger. Our results suggest that there is a strong correlation 
between cell stiffness and cell-induced microstreaming flow. Future work will focus on characterizing our platform in order to calibrate our system as a label-free elastography technique, as well as on investigating the relationship between the microstreaming effect and the resonant characteristics of cells.

\subsection{Methods}

\subsubsection{Device Fabrication}

We designed and fabricated two microfluidic channels with a width of $1000 \mu \mathrm{m}$ and heights of $25 \mu \mathrm{m}$ and $85 \mu \mathrm{m}$. Both channels were patterned on a silicon wafer using standard photolithography method. The PDMS replicates of the channels were then fabricated using soft lithography technique. ${ }^{166}$ Briefly, Sylgard 184 silicone elastomer base and curing agent (Dow Corning) were mixed at a mixing ratio of $10: 1$ and cured at $70^{\circ} \mathrm{C}$ for $2 \mathrm{hr}$. The cross-linked PDMS device was then peeled off the wafer, the excess PDMS was cut such that a thin wall is remained for the channel, and the inlets were punched using $1 \mathrm{~mm}$ biopsy punch (Integra Miltex). A permanent bonding between the surfaces of PDMS device and glass substrate was achieved after the two surfaces were functionalized by oxygen plasma (PDC32G, Harrick Plasma) for $30 \mathrm{~s}$ and then touched firmly. We then placed an acoustic transducer (7BB-27-4L0, Mouser Electronics) on the glass substrate close to the channel. The transducer was bonded permanently to the glass substrate using liquid PDMS prepared as mentioned above. The following glass substrates were used in the experiments: $\sim 100 \mu \mathrm{m}$ thick (No. 0 cover glass, Ted Pella), $\sim 140 \mu \mathrm{m}$ thick (No. 1 cover glass, VWR), $\sim 220 \mu \mathrm{m}$ thick (No. 2 cover glass, Ted Pella), and $\sim 1000 \mu \mathrm{m}$ thick (2590 glass slide, VWR).

\subsubsection{Device Operation}

The acoustofluidic device was mounted on the stage of an inverted microscope. Actuation voltage and frequency were generated using a function generator (Wavetek) and amplified (when needed) using an amplifier ( $\mathrm{T} \& \mathrm{C}$ Power Conversion). Acoustic microstreaming flows were captured using a high-speed camera (Vision Research). 


\subsubsection{Cells Preparation}

Microfluidic channels were incubated with $50 \mu \mathrm{gr} \mathrm{mL}^{-1}$ fibronectin (SIGMA) for $2 \mathrm{hr}$ before MDA-MB-231 and MCF-7 cells suspended in culture medium were injected to their respected channels. The cell culture medium is composed of RPMI 1640 (Wisent Bioproducts) growth medium supplemented with 10\% (v/v) fetal bovine serum (Wisent Bioproducts) and 1\% (v/v) PenStrep (Wisent Bioproducts). We then incubated the devices containing cells at $37^{\circ} \mathrm{C}$ overnight so that cells settle and adhere to the substrates. Confocal images of cell cytoskeletal structure are taken by staining actin filaments with Alexa Fluor 488 Phalloidin (Invitrogen) and CellLight Actin-GFP (Invitrogen).

\subsubsection{Cell Treatments}

All treatments were conducted on MDA-MB-231 cells. We measured the streaming flow by capturing $4 \mathrm{~s}$ videos of the tracers surrounding single-cells. For the trypsinization experiment, we incubated cells with $0.25 \%$ Trypsin-EDTA (Wisent Bioproducts) for $4 \mathrm{~min}$. Then, the trypsin solution is replaced with the culture medium, and the cells were incubated at $37^{\circ} \mathrm{C}$ for $24 \mathrm{hr}$. For conducting osmotic shock experiments, we prepared four solutions of culture medium with $0 \%$ (v/v) DI, 30\% DI, 70\%, and 100\% DI (no culture medium). The total duration of osmotic shock experiment was less than 10 min during which all four solutions were injected successively into the microfluidic channel with 2 min intervals in between. The 100\% DI solution was then replaced with the culture medium, and the cells were incubated at $37^{\circ} \mathrm{C}$ for $1 \mathrm{hr}$. The drug solution used

for targeting actin filaments was composed of $4 \mu \mathrm{M}$ Cytochalasin D (Bioshop) in cell culture medium. The actin filament disruption experiment was conducted by introducing the drug solution into the channel and then incubating at $37^{\circ} \mathrm{C}$ for $1 \mathrm{hr}$. The drug solution was then replaced with the culture medium, and the cells were incubated at $37^{\circ} \mathrm{C}$ for $5 \mathrm{hr}$. Cell fixation was achieved by replacing the culture medium with 4\% Paraformaldehyde solution and then incubating at room temperature for $30 \mathrm{~min} .10 \mu \mathrm{M}$ Nocodazole (Cell Signaling Technology) in cell culture medium was used to disrupt microtubulins. This treatment was conducted by incubating the cells with the Nocodazole solution at $37^{\circ} \mathrm{C}$ for $1 \mathrm{hr}$. 


\subsubsection{Atomic Force Microscopy (AFM) Measurements}

To measure cell elastic (Young's) moduli E, AFM measurements were performed with MDA-MB-231 cells in cell culture medium (Wisent Bioproducts) using an AFM system (JPK NanoWizard ${ }^{\circledR} 4$, Bruker). Tipless silicon nitride cantilever force probes (Bruker) with a spring constant of $0.06 \mathrm{~N} \mathrm{~m}^{-1}$ and a resonating frequency of $18 \mathrm{kHz}$ were customized to perform indentation force-distance measurements. Customization was done by attaching a standard bead with $10 \mu \mathrm{m}$ diameter (Whitehouse Scientific) to the apex of each force probe using an adhesive that is cured under UV light (Loctite ${ }^{\circledR}$ AA $349^{\mathrm{TM}}$ IMPRUV () ). All tips were calibrated with contact-based and contact-free methods according to the manufacturer's instructions. Set point $(1.05 \mathrm{nN})$, relative set point $(2.101 \mathrm{nN})$, z-length $(5 \mu \mathrm{m})$, and extend speed $\left(5 \mu \mathrm{m} \mathrm{s}^{-1}\right)$ were kept constant. Per each experimental group, four cells were indented on their cell body immediately adjacent to the position of the nucleus. Data obtained from 500 indentations per cell were processed using JPK Data Processing Software (Bruker) by fitting the resulting force curves using an established Hertz model as described previously. ${ }^{241}$ Mean values were calculated from all processed measurements and plotted as mean Young's elastic modulus ( \pm standard deviation). 


\section{Intracellular}

Delivery

Using

\section{Acoustofluidics}

The work presented in this chapter is based on the following manuscript, which is not published yet.

Salari, A.,* Appak-Baskoy, S., Coe, I., Abousawan, J., Antonescu, C., Tsai, S.S.H., and Kolios, M.C. Intracellular delivery using acoustofluidics. In Preparation.

\section{Author's Contribution}

For the article presented here, the author wrote the majority of the manuscript, designed, fabricated, and tested the devices, conducted all experiments, and collected and analyzed data. S. Appak-Baskoy helped with providing chemicals and optimized protocols for conducting staining and gene delivery, discussions, and writing the manuscript. J. Abousawan and C. Antonescu provided a cell line and discussions. I. Coe provided equipment, discussion, and supervision.

\subsection{Introduction}

Targeted drug delivery, gene silencing, and DNA editing technologies, as well as intracellular imaging, rely on the successful delivery of substances into cells. Among these substances are genetic materials, synthetic materials, natural substances, drugs, and drug carriers. In an intact plasma membrane, small size molecules (smaller than $500 \mathrm{Da}$ ) may pass the membrane 
through membrane protein transporters and channels, ${ }^{19,242}$ however, larger substances can enter via substance engulfment in intracellular-bound vesicles made up of the cellular membrane, known as endocytic vesicles, through a process called endocytosis. ${ }^{243}$

For effective delivery of materials into the cell two general approaches are used: a) biochemical methods, which allow overcoming the barrier of the polar hydrophobic lipid bilayer, such as calcium phosphate transfection or lipofection, and b) physical methods, aimed at transient disruption of the cellular membrane by introducing pores such as electroporation and sonoporation. Biochemical methods, including insoluble calcium phosphate precipitation of DNA or transfection by lipofection, introduce genetic materials to the cell by means of endocytosis. Due to the lower efficacy of the calcium phosphate precipitation method, lipid-based transfection methods are considered the "gold-standard" and are routinely used as a basis of comparison to evaluate the efficiencies of alternative methods. ${ }^{244}$ Lipofection leads to the association of charge-neutralized liposome-cargo complexes with the negative-charged plasma membrane and the eventual delivery of cargo to the cell interior. ${ }^{245}$ Although lipofection effectively delivers materials to the cells, it still has significant drawbacks as transfection efficacy differs between different cells and even within the same population of isogenic cells.

On the other hand, physical methods, such as electroporation, rely on external forces. These external forces cause the formation of physical pores in the lipid bilayer that leads to immediate transport of materials across the membrane. ${ }^{19}$ However, the mechanisms underlying these techniques may cause permanent permeabilization of the membrane and the consequent loss of cell homeostasis. ${ }^{246}$ In sonoporation, for instance, microbubble inertial cavitation can cause small pores on the membrane that facilitates the entry of small molecules through diffusion. However, larger substances, like genetic materials, may need to pass the cell through larger pores that need stronger ultrasound waves, which may produce larger hard-to-seal pores on the membrane leading to cell death. ${ }^{247}$

Most membrane disruption-mediated methods, regardless of their underlying delivery mechanism being biochemical or physical, suffer from low cellular viability when it comes to a high-efficiency delivery. The reason for this low viability is the fact that these disruption strategies are typically unable to account for the cellular heterogeneity in tolerating the external forces. As a 
result, the disruption methodology is usually engineered to be harsh enough so that a larger number of cells experience the minimum membrane disruption of sufficient size and lifetime needed for the passage of the cargo material. For example, in the microfluidic cell squeezing technique, due to the fixed constriction geometry, the shear stress imposed by the channel walls on cells with various sizes can differ by orders of magnitudes. ${ }^{43}$

Other physical methods, including microinjection, that feature a higher precision in the amount of delivery, are typically low-throughput and labor-intensive. This can dramatically limit their applications. Therefore, robust and high-throughput strategies are needed in order to avoid cellular damage and facilitate efficient intracellular delivery of various substances. Recently, we reported an acoustofluidic platform for monitoring cell-induced acoustic microstreaming and probing cellular stiffness by actuating cells via acoustic waves. ${ }^{201}$ Here, using a similar methodology, we propose a novel strategy for intracellular delivery, where adherent cells are forcefully oscillated using acoustics. We find that, as a result of acoustic excitation and the resultant microstreaming flow, cellular uptake occurs through endocytic pathways. We show that this methodology has unique characteristics in delivering a precise amount of cargo materials into the cells regardless of the size and charge of the cargo molecule while maintaining high viability (above 91\%). In addition, due to the nature of this acoustofluidic platform, it enables a highthroughput delivery strategy with treatment time and actuation amplitude regarded as the control parameters for delivery dosage. We demonstrate successful delivery of $500 \mathrm{kDa}$ dextran to various cell lines with an unprecedented ${ }^{43,91,248,249}$ delivery efficiency in the range of $65-85 \%$ within only a $20 \mathrm{~min}$ of treatment. We also identify endocytosis as the dominant mechanism of delivery of substances and demonstrate the applicability of our technique, not only for drug delivery but also for efficient delivery of siRNA and plasmids for gene therapy.

\subsection{Results}

\subsubsection{Working Principle}

As displayed in Figure 6.1, our acoustofluidic device is composed of a single-layer polydimethylsiloxane (PDMS) microfluidic channel and a piezoelectric (PZT) transducer, which bonded onto a thin glass substrate. To maximize the contribution of Lamb waves in the propagation 
of sound waves through the substrate, we select a substrate with a thickness of $\sim 70 \mu \mathrm{m} .{ }^{71}$ The surface of the microfluidic channel is functionalized using fibronectin solution at a concentration of $100 \mu \mathrm{g} \mathrm{mL}^{-1}$ to facilitate the adhesion of cells onto the glass surface. The cells are then seeded into the microfluidic channel and are cultured for at least two days before treatment with acoustics. By applying square-wave voltages to the transducer at a constant frequency of $96 \mathrm{kHz}$ and using different amplitudes, we explore the delivery of cargo materials into numerous adherent cell lines for various treatment duration. During the treatment, cells are exposed to fluorescently labeled cargo materials mixed with the cell culture medium. Once the treatment is over, the cells are washed before the fluorescent images are taken. In control and treatment experiments, cells are exposed to identical solutions (containing cargo at an equal concentration) for an equal amount of time. Image analysis techniques are then employed to quantify the delivery efficiency and the uptake using a semi-automated protocol (see Note E.1). Briefly, the delivery efficiency is defined as the number of successfully treated cells divided by the total number of treated cells. The cells with a fluorescence intensity higher than the mean intensity of $95 \%$ of the control cells are considered as successfully treated cells. ${ }^{42,43}$

The acoustic pressure distribution on a substrate surface can vary depending on the dimensions of the substrate, frequency of actuation, and the location of the PZT transducer on the substrate. Assuming the area covered by each cell is approximately $\sim 50 \times 50 \mu \mathrm{m}$, a total of 50,000 cells in a confluent monolayer fitted within a region of interest (ROI) located on pressure antinodal regions can be treated simultaneously. Substrates with larger areas and numerous ROIs can be used for treating hundreds of thousands of cells in a single run. For the sake of simplicity and as a proof-of-concept demonstration, in this chapter, we choose an ROI of $2 \times 2 \mathrm{~mm}$. Adhered cells (a total of $\sim 100-200$ cells per experiment) within the ROI are monitored before and after the treatments to quantify the uptake. 


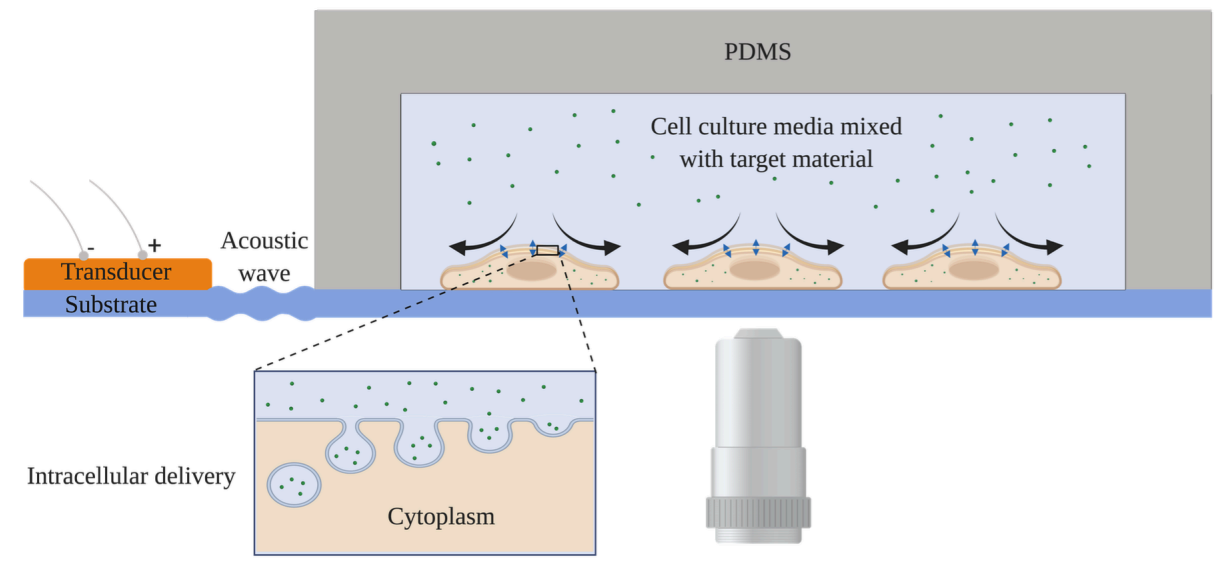

Figure 6.1 Schematic of the acoustofluidic device showing the hypothetical mechanism of intracellular delivery. Due to the actuation of the PZT transducer, acoustic waves are generated and propagated through the substrate. The excitation of cells induces the formation of acoustic microstreaming, which in turn facilitates the transport of cargo material from the bulk of the liquid to the cell membrane. As a result of the combined effect of acoustic excitation and microstreaming flow, the cargo material is taken up by the cell. The schematic is created with Biorender.com.

\subsubsection{Effect of Different Actuation Parameters}

To evaluate the relationship between the efficiency of uptake and the actuation parameters, we aim to deliver relatively small molecules (i.e., $3 \mathrm{kDa}$ dextran conjugated with Alexa Fluor 488) into MDA-MB-231 cells. Dextran is a hydrophilic and non-digestible carbohydrate commercially available in a wide range of molecular sizes and photostable fluorophore conjugates with welldifferentiated excitation/emission spectra suitable for fluorescence microscopy. ${ }^{250}$ Figure 6.2 shows the delivery results for different treatment duration and actuation power. We found that 1 $\mathrm{W}$ is the maximum input power possible for treating cells with continuous sound waves while avoiding excessive heat generation by the transducer. By increasing the treatment duration from 2 min to $20 \mathrm{~min}$, a $\sim$ six-fold increase in the delivery efficiency (Figure 6.2a) and a $\sim$ three-fold increase in the amount of delivery (Figure E.2a) are obtained. Lowering the input power to below $1 \mathrm{~W}$ causes the cell oscillations to be weaker, and therefore, decreases the delivery efficiency (Figure 6.2b) and the amount of delivery (Figure E.2b). Although by increasing the input power to values above $1 \mathrm{~W}$, the delivery efficiency keeps increasing, heat generation by the transducer can negatively impact the cellular viability. As a result, for the input powers above $1 \mathrm{~W}$, we choose 
to use pulses instead of continuous waves. The duty cycles (written as percentage values in Figure $6.2 \mathrm{c}$ and Figure E.2c), therefore, are adjusted such that the maximum temperature within the ROI during the treatment remains below $37^{\circ} \mathrm{C}$. For cells treated with $21 \mathrm{~W}$ and $7 \%$ duty cycle, the amount of delivery appears to be nonuniform. In some cells (indicated by white arrows in Figure 6.2d), dextran is distributed homogenously throughout their entire cytosol, whereas other cells exhibit fluorescence from localized spots in their cytosols. This observation can be explained by considering the acoustic excitation that cells experience, as a result of high actuation power (i.e., $21 \mathrm{~W}$ ) used. Based on our observations, these strong oscillations can cause the cells to lose some of their adhesion focal points such that, by the end of the treatment, there will be some cells that are loosely attached to the substrate surface. This forceful detachment (similar to the scrape loading technique $)^{4,251}$ can yield plasma membrane disruption, which in turn can cause the cargo material to be internalized through transient openings on the plasma membrane.

We also find that, at low power actuation $(\leq 1 \mathrm{~W})$, no cell death due to acoustic excitation occurs, as almost all cells maintain their adhesion to the channel surface for the entire duration of treatment. However, a 9\% cell death (corresponds to a $91 \%$ viability) is observed when very high actuation powers (e.g., $200 \mathrm{~W}$ ) are applied, which causes the entire cell population to detach from the substrate surface in less than a second. This unique feature of our acoustofluidic strategy in maintaining a high viability rate is due to the way sound waves are introduced and transmitted in our device. As opposed to bulk ultrasound treatments, where acoustic energy is introduced to all cells for the entire duration of treatment, in our acoustofluidic strategy, only the attached cells are exposed to the incoming sound waves, and as soon as a cell is detached, the acoustic energy experienced by the cell drops considerably. To ensure that a continuous microstreaming flow is maintained throughout the entire treatment period and that the membrane disruption is minimized while a high delivery efficiency is achieved, we used $1 \mathrm{~W}$ actuation power and 20 min treatment duration for the rest of the experiments. 


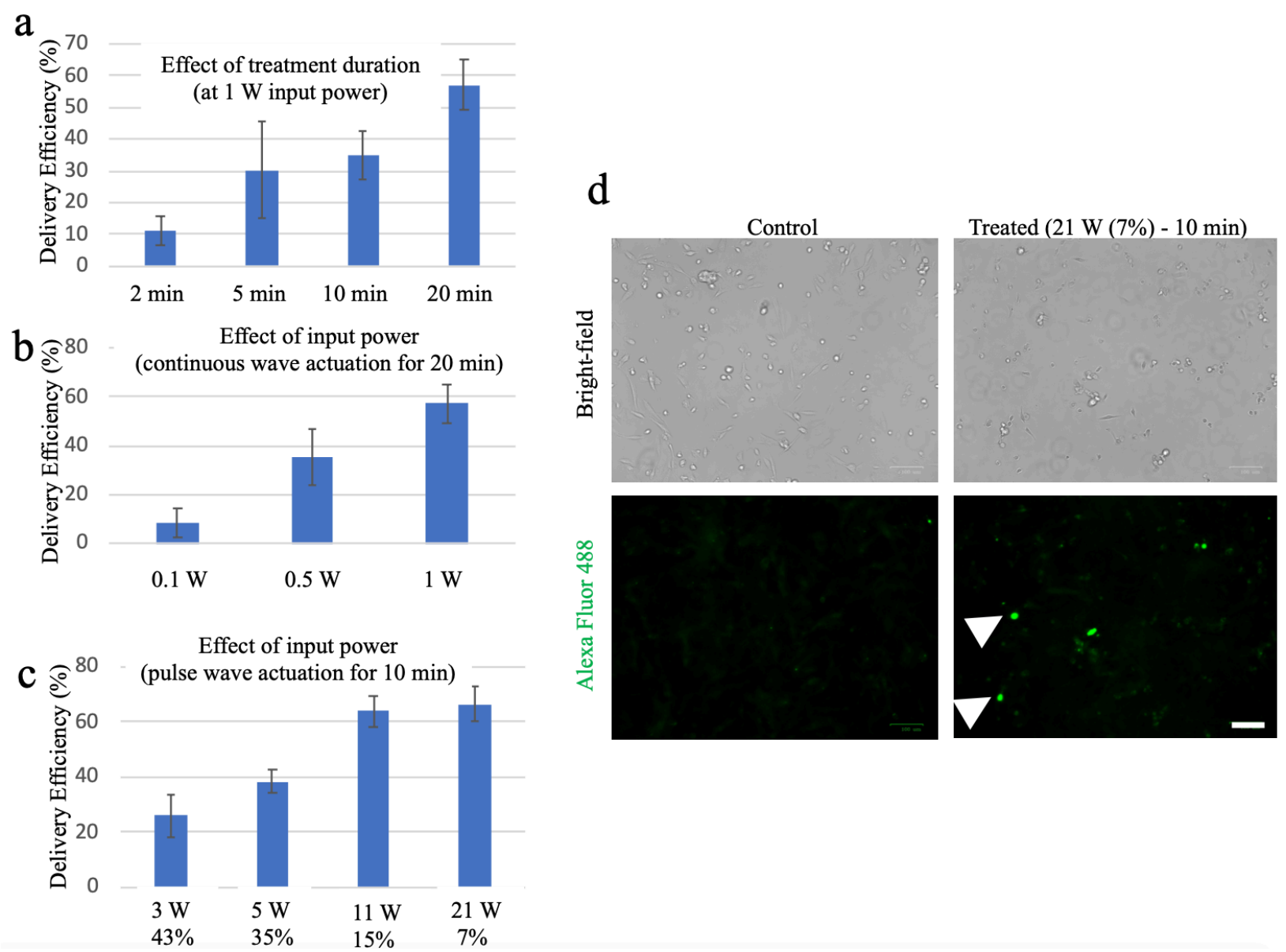

Figure 6.2 The effect of input power and treatment duration on the intracellular delivery. (a) The delivery results for different treatment duration when continuous $1 \mathrm{~W}$ input power is applied. (b) Delivery results for the input power in the range of $0.1-1 \mathrm{~W}$ and $20 \mathrm{~min}$ treatment. Within this range, the temperature rise is insignificant, and therefore, a continuous wave actuation is used. (c) Delivery results for different input powers in the range of 3-21 W. For each input power, the duty cycle of the square-waves is set in the range of $7-43 \%$ for 10 min treatment duration, to avoid excessive heat generated by the transducer and the vibrating PDMS. (d) Bright-field and fluorescent images showing the cellular uptake in control and treated cells. The white arrows point to individual cells as examples of those receiving cargo material as a result of disruptions to the cell membrane. In all experiments, $3 \mathrm{kDa}$ dextran is used as the cargo material for delivery into MDA-MB-231 cells. The scale bar represents $100 \mu \mathrm{m}$. The error bars indicate the standard deviation of three independent experiments. 


\subsubsection{Versatility in the Delivery of Various Materials into Different Cells}

Next, we study the versatility of our acoustofluidic technique in delivering larger size cargo materials, including $70 \mathrm{kDa}$ anionic fluorescein isothiocyanate dextran (FITC-dextran) and 500 $\mathrm{kDa}$ anionic fluorescein dextran. As depicted in Figure 6.3a, a delivery efficiency of $\sim 60-85 \%$ is obtained for 3-500 kDa dextran, with the highest efficiency for $500 \mathrm{kDa}$ dextran. This evidence supports the hypothesis that membrane disruption cannot be the dominant mechanism of delivery for $500 \mathrm{kDa}$ dextran, because if it was, then a higher amount of delivery (see Figure E.3a) compared to their respective control would occur for smaller size dextran molecules due to their higher diffusion rate. As shown in Figure $6.3 \mathrm{~b}$ and Figure E.3c-d, our intracellular delivery technique is not limited to MDA-MB-231 cells, as we also demonstrate successful delivery of dextran to MCF-7 and PC3 cells. 


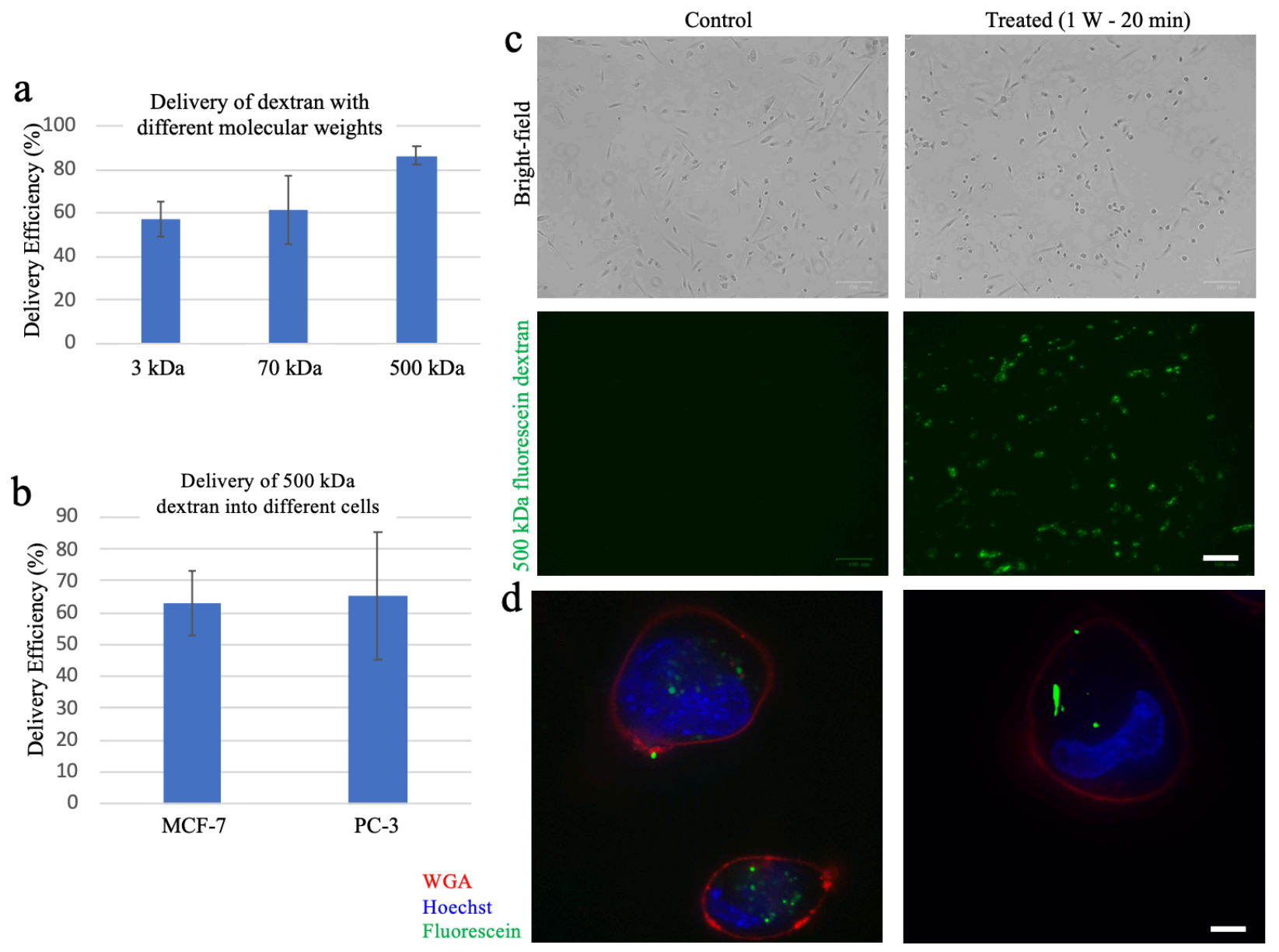

Figure 6.3 The versatility of intracellular delivery of dextran with various sizes into different cells.

(a) Delivery results for dextran with different molecular weights into MDA-MB-231 cells. (b) Delivery results for MCF-7 and PC3 cells. Delivery efficiency of $\sim 63 \%$ is obtained in both experiments. (c) Bright-field and fluorescent images showing $500 \mathrm{kDa}$ dextran delivery into MDAMB-231 cells. The presence of localized high-intensity aggregates of fluorescein dextran suggests an endocytic-dominant mechanism of delivery. (d) Confocal images of living MDA-MB-231 cells exhibiting the uptake of fluorescein-conjugated $500 \mathrm{kDa}$ dextran. Plasma membrane and cell nucleus are stained with wheat germ agglutinin (WGA) and Hoechst 33342, respectively. The scale bars represent $100 \mu \mathrm{m}$ in (c) and $20 \mu \mathrm{m}$ in (d). A 20 min continuous square-wave at $1 \mathrm{~W}$ input power is used in all experiments. The error bars indicate the standard deviation of three independent experiments. 


\subsubsection{Probing the Mechanism of Intracellular Delivery}

The outer leaflet of the plasma membrane is the surface through which each cell communicates with its environment, and to appropriately respond to, or affect, its environment, its composition must be tightly regulated by the cell. In a process known as endocytosis, plasma membrane lipids, integral proteins, and extracellular fluid become fully internalized into the cell. ${ }^{252}$ There are many pathways of endocytosis orchestrated by different proteins at the cell surface that operate simultaneously. The most well-known of these pathways is the clathrin-mediated endocytosis. Other endocytic pathways that are not dependent on the protein clathrin are known as clathrin-independent pathways that involve proteins, such as caveolin and flotillin. Cells continuously take up fluid from their neighborhood by a process designated as fluid-phase endocytosis. Unlike other types of uptake, this kind of endocytosis is not preceded by specific binding to the plasma membrane or concentration of molecules at particular sites. ${ }^{253} \mathrm{~A}$ variety of fluorescently labeled dextran molecules are commonly used as markers of fluid-phase endocytosis and macropinocytic probes to study the properties of endocytic cargoes. ${ }^{254}$

In contrast to $3 \mathrm{kDa}$ dextran molecules that can diffuse into the cell cytosol if the cell membrane is disrupted (Figure 6.2d), $500 \mathrm{kDa}$ dextran molecules appear to be localized as aggregates within the cell cytosol (Figure 6.3c and d). This could be an indication that $500 \mathrm{kDa}$ dextran is taken up by the cell via an endocytosis-dominant mechanism that is enhanced as a result of acoustic excitation. Massive endocytosis, as well as exocytosis, are both associated with the intracellular increase of calcium ions $\left(\mathrm{Ca}^{+2}\right)$. Therefore, we measure the intracellular level of $\mathrm{Ca}^{+2}$ using Fluo-4 AM, which is a labeled cell-permeant indicator of $\mathrm{Ca}^{+2}$. As depicted in Figure $6.4 \mathrm{a}$, a significant increase in $\mathrm{Ca}^{+2}$ level is observed in the treated cells, which can be linked to the endocytic activity of cells. ${ }^{255,256}$ As calcium influx can trigger endocytosis, ${ }^{257}$ depleting $\mathrm{Ca}^{+2}$ from the culture medium should decrease the amount of intracellular delivery. To verify this, we treat cells with dextran in calcium-free phosphate-buffered saline (PBS) solutions. Our results show that the delivery efficiency is not suppressed (Figure 6.4b) and a $\sim 40 \%$ decrease in the uptake (Figure E.4a) is observed when cells are treated in a calcium-free solution.

When extracellular materials are internalized through an endocytic pathway, they can be entrapped in endosomes before degraded by enzymes in lysosomes. ${ }^{258}$ Using pHrodo Green 10 
$\mathrm{kDa}$ dextran, which is a $\mathrm{pH}$-sensitive dye, we monitor the uptake of dextran through endosomes. Due to its $\mathrm{pH}$ sensitivity, this dye exhibits a dramatic increase in fluorescence when $\mathrm{pH}$ decreases from neutral to acidic values. Delivery efficiency of $\sim 50 \%$ obtained for cells treated with this dye (Figure 6.4c and E.4b) is evidence of the role of the endosomal pathway in the intracellular delivery. As opposed to conventional fluorescently labeled dextran molecules, which are anionic, we also attempt to deliver zwitterionic dextran to MDA-MB-231 at an efficiency of $\sim 60 \%$. An increase in the amount of delivery of zwitterionic dextran compared to anionic dextran (Figure E.3b) could be due to the polar characteristics of the lipid bilayer that makes the plasma membrane impermeable to charged molecules (regardless of the molecular size) and more permeable to uncharged ones. ${ }^{259}$

It has been shown that treatment with ultrasound and microbubbles can enhance flotillindependent fluid-phase endocytosis and massive endocytosis. ${ }^{260}$ We hypothesize that similar pathways could be triggered in our device, and therefore, we also study the amount of delivery through flotillin-mediated endocytosis. We acoustically treat healthy MDA-MB-231 cells and transfected ones, where flotillin-1 protein is knocked out by CRISPR/Cas9. As illustrated in Figure E.4c, even though the flotillin-knockout cells exhibit a $\sim 40 \%$ decrease in the uptake of $70 \mathrm{kDa}$ dextran compared to the healthy ones, the uptake is still significantly higher than the control cells. This finding shows the role of flotillin-mediated endocytosis in the delivery of $70 \mathrm{kDa}$ dextran.

In our acoustofluidic device, cell oscillations generate shear stress on the viscous boundary layer surrounding the plasma membrane, which in turn, drags the bulk of the fluid into motion. ${ }^{201}$ Based on Newton's third law, the plasma membrane, too, has to experience shear stress with equal magnitude. Since fluid shear forces alone can cause membrane permeabilization, ${ }^{19}$ we calculate the shear stress exerted on the plasma membrane when the cell is actuated at $1 \mathrm{~W}$. Then, using a flow control system, we expose the adherent cells to a unidirectional flow that generates roughly equal shear stress on the cell monolayer. As illustrated in Figure 6.4d-e, treatment with shear stress due to the unidirectional flow (when there are no acoustic excitation) can contribute a maximum of $20 \%$ to the delivery efficiency of $500 \mathrm{kDa}$ dextran. The amount of uptake is also much lower in the shear-only treated cells compared to the acoustically-treated ones (Figure E.4d). However, this 
shear stress could be responsible to some extent for the elevated calcium level shown in Figure $6.4 a^{261,262}$

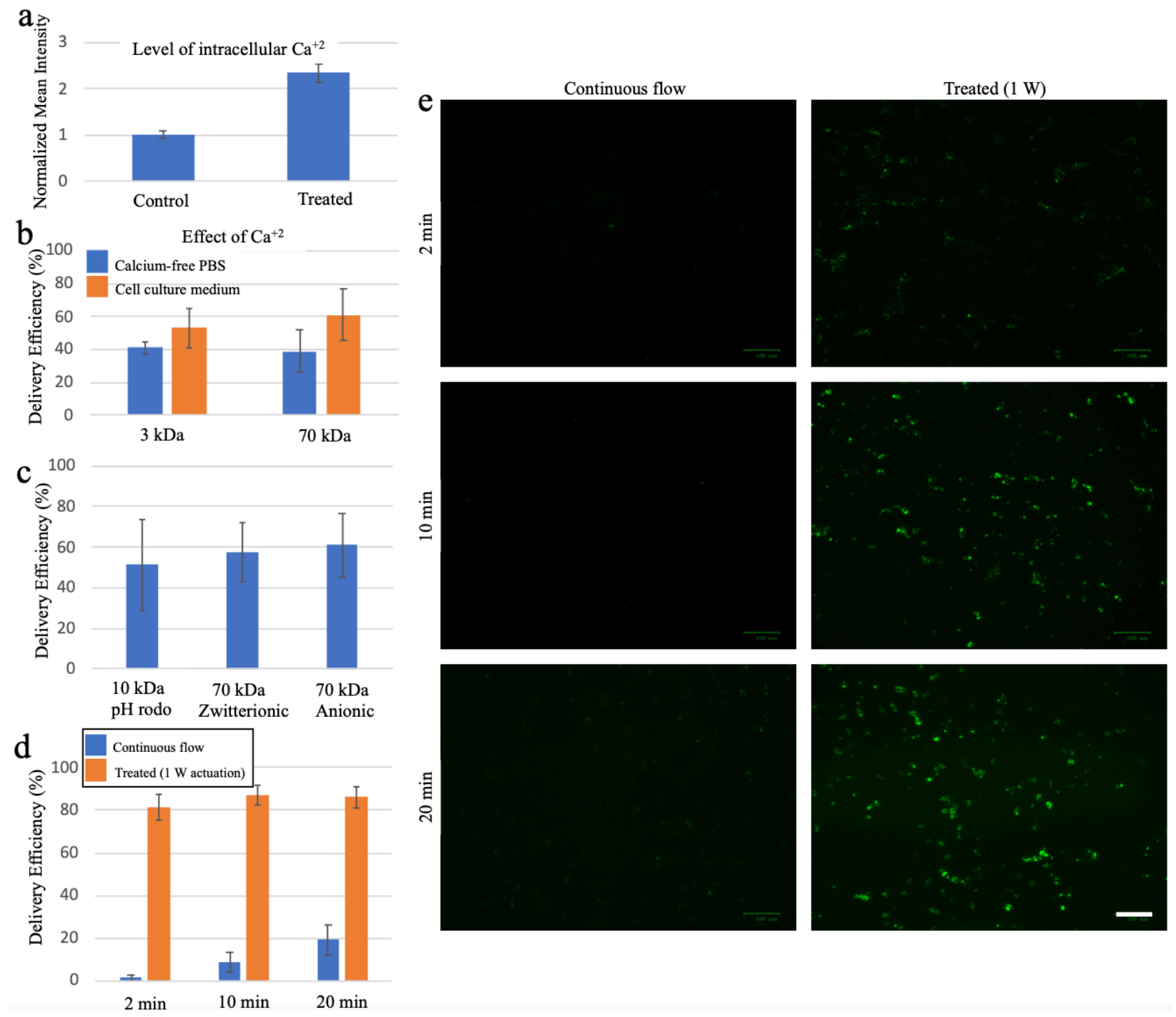

Figure 6.4 Probing possible mechanisms of delivery. (a) The intracellular level of Fluo-4 AM, a labeled calcium indicator, in control and treated cells. (b) Effect of $\mathrm{Ca}^{+2}$ on the delivery of $3 \mathrm{kDa}$ and $70 \mathrm{kDa}$ dextran. (c) Delivery results of dextran with different charges and a $\mathrm{pH}$-sensitive fluorescently labeled dextran (i.e., pHrodo Green $10 \mathrm{kDa}$ dextran), which fluoresces at low $\mathrm{pH}$ values. (d) Exposure to continuous flow (shear stress only) and acoustic excitation on the delivery efficiency for different exposure durations. Acoustically actuated cells exhibit significantly higher delivery efficiencies compared to those exposed to shear stress only. (e) Fluorescent images of the cells acoustically treated at $1 \mathrm{~W}$ and those exposed to the continuous flow. The scale bar represents 
$100 \mu \mathrm{m}$. Acoustic treatments are conducted on MDA-MB-231 cells using a continuous squarewave at $1 \mathrm{~W}$ input power. Results shown in $(\mathrm{a}-\mathrm{c})$ are for $20 \mathrm{~min}$ acoustic treatments. The error bars indicate the standard deviation of three independent experiments.

\subsubsection{Delivery of Nucleic Acids}

One of the most commonly used tools in molecular biology for inhibiting the expression of certain genes is the silencing of the RNA that interferes with both translation and transcription of target genes, leading to repressed protein expression. The discovery of RNA interference is a milestone in gene therapy because it opens opportunities to discover numerous pathways in many diseases including cancer, genetic disorders, autoimmune diseases, and viral infections. ${ }^{263}$ siRNAs are double-stranded RNA molecules of 20-25 base pairs in length. Effective cytosolic delivery of siRNA is a critical first step to achieve the desired protein expression inhibition. Conventional siRNA delivery methods are biochemical ones in which siRNA and lipid molecules make a complex that is easily taken by the semi-permeable bilayer lipid membrane via a process called lipofection. ${ }^{245}$ To further explore the applicability of our acoustofluidic device for gene therapy, we treat MDA-MB-231 cells with labeled GAPDH siRNA, which results in a $\sim 45 \%$ delivery efficiency of siRNA (Figure 6.5a-b). Compared to the control experiment (lipofection), the acoustically-treated cells exhibit a $\sim$ ten-fold increase in the uptake of siRNA after 20 minutes of treatment (Figure E.5a).

Recombinant DNA technologies allow obtaining pure DNA samples from genomes, which are too large to analyze in detail at the molecular level directly. With the help of these molecular biology technologies, almost any gene can be purified, its sequence determined, and the functional regions of the sequence explored by altering it in planned ways and reintroducing the DNA into cells and whole organisms. ${ }^{264}$ One of the most commonly used tools in molecular biology to introduce DNA into cells are plasmid vectors. Plasmids are circular, double-stranded DNA molecules that occur naturally in bacteria, yeast, and some higher eukaryotic cells and exist in a parasitic or symbiotic relationship with their host cell. ${ }^{264}$ Plasmids can be engineered to possess recombinant DNA and to transfer genes between different cells. They are also used to introduce CRISPR/Cas9 gene-editing tools into the cells with either physical techniques, such as electroporation, or biochemical techniques, such as lipofectamine transfection. It has been shown 
that introducing Cas9-sgRNA complexes directly to the cells via electroporation, instead of using chemical-based methods, can improve efficiency and reduce off-target effects, highlighting a trend towards direct delivery of macromolecules. ${ }^{18,265}$ Plasmids are usually 5-10 kilo-base pairs with a diameter of smaller than $100 \mathrm{~nm} .^{266,267}$ Since it carries nucleotides with phosphate groups, the overall net charge of the plasmid is negative; therefore, cationic compounds, such as lipids and polymer reagents, condense plasmids into solid nanoparticles with dimensions of tens of nanometers easing the uptake of the plasmid by shrinking its size and shielding its negative charge. ${ }^{19,268}$ Plasmids often carry specific markers, such as green fluorescent protein (GFP), for selection, and generally, plasmids that carry empty vector green fluorescent protein (GFP) are used. In this study, we show the intracellular delivery of empty vector EGFP carrying plasmid that is often used for assessing the transfection efficacy. Compared to the lipofectamine transfection, treatment with acoustics and lipofection shows a delivery efficiency of $\sim 33 \%$ (Figure 6.5 ) and a $\sim$ three-fold increase in GFP intensity (Figure E.5b).

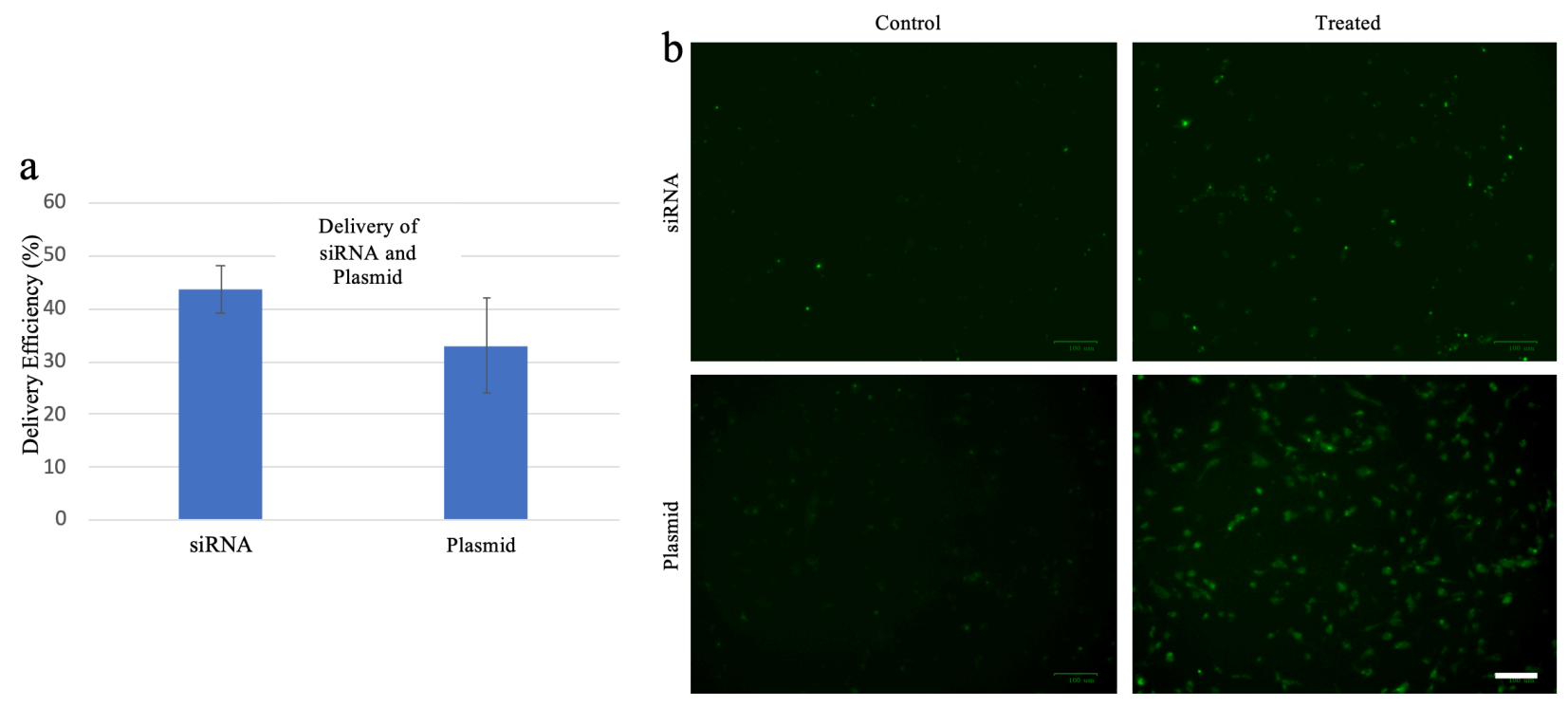

Figure 6.5 Intracellular delivery of nucleic acids. (a) Delivery results of siRNA and plasmid. (b) Fluorescent images of the delivered siRNA and plasmid for control and treated cells. The scale bar represents $100 \mu \mathrm{m}$ in (b). Treatments are conducted on MDA-MB-231 cells using a $20 \mathrm{~min}$ continuous square-wave at $1 \mathrm{~W}$ input power. The error bars indicate the standard deviation of three independent experiments. 


\subsection{Discussion}

In this chapter, we showed that acoustofluidic treatment could induce endocytosis of different sizes of dextran molecules ( $3 \mathrm{kDa}, 70 \mathrm{kDa}$, and $500 \mathrm{kDa}$ ) efficiently in a relatively short time (on the order of minutes) and with high cellular viability. We demonstrated successful delivery of $500 \mathrm{kDa}$ dextran to various cell lines with an unprecedented ${ }^{43,91,248,249}$ delivery efficiency in the range of $65-85 \%$ within a short $(\leq 20 \mathrm{~min}$ ) treatment duration. The acidity of vesicles increases as they form and through the maturation process. ${ }^{269,270}$ An enhanced fluorescence intensity of pHrodo Green $10 \mathrm{kDa}$ dextran after the treatment indicates that more endosomes are formed when $10 \mathrm{kDa}$ dextran is taken up by the acoustically excited cells compared to the control cells.

The uptake of $70 \mathrm{kDa}$ dextran by the flotillin-knockout cells upon acoustic excitation shows that the intracellular delivery may also depend on other pathways including those mediated by clathrin or caveolin. We also observed that in our acoustofluidic technique, due to the generation of microstreaming flow, dextran compounds are convected towards the cellular membrane. The larger the substance is, the more time is needed for its internalization. The substances that are drawn to the membrane do not dissociate from the membrane even after washing, suggesting that these molecules are in the process of getting internalized at the cellular membrane. ${ }^{271}$

For efficient intracellular delivery, the molecular charge is an important consideration as molecules with a positive charge, such as cationic lipids and cationic cell-penetrating peptides and polymers commonly used as transfection agents, tend to be more efficient at penetrating the negatively charged plasma membrane for gaining entry to cells. ${ }^{19,272}$ Here, we showed that acoustic excitation could deliver substances, regardless of their molecular charges, as it enables the delivery of neutral and anionic molecules. Therefore, our acoustofluidic strategy could also be used in the intracellular delivery of proteins with negative charges, such as antibodies. Most of the typical cargo molecules, including DNA and RNA, possess a net negative charge. Therefore, an effective delivery method would be either a direct method such as the physical methods or a biochemical method in which the charge similarity between the cellular membrane and cargo molecules could be altered. 
In this study, we also showed that treatment with low amplitude acoustics together with lipofection could deliver fluorescently labeled GAPDH siRNA to the cytoplasm of the MDA-MB231 cells more effectively than the conventional biochemical delivery method carried out by lipofection alone. Moreover, treatment in our acoustofluidic device also decreases the time needed for lipofection as it was able to deliver GAPDH siRNA in as short as 20 minutes treatment. Importantly, a combination of acoustic excitation with lipofectamine minimizes the harmful effects of lipofection on the cells as the cells are exposed to the complex for a shorter time than the conventional 4-6-hour time window.

The intracellular delivery using acoustics, together with lipofectamine, enhanced the expression of EGFP by the plasmid (almost three-fold increase, Figure E.5b) within $1 \mathrm{hr}$ compared to the conventional lipofectamine based plasmid delivery.

The design of the acoustofluidic platform presented in this chapter can be further developed for treating a larger population of cells in a single run. In addition, treating cells at their resonant frequencies ${ }^{273,274}$ and delivery to hard-to-transfect cells can be the interesting future directions of our work.

\subsection{Methods}

\subsubsection{Acoustofluidic Device Fabrication}

We used photolithography followed by soft lithography to fabricate a PDMS microfluidic channel. The channel and $\mathrm{a} \sim 70 \mu \mathrm{m}$ thick glass substrate (Agar Scientific) were permanently bonded after plasma treatment. A PZT transducer (7BB-27-4L0, Mouser Electronics) was then bonded onto the glass substrate next to the microfluidic channel using liquid PDMS. Actuation voltage and frequency were generated using a function generator (Siglent) and amplified using an amplifier (E\&I). Acoustic microstreaming flows were captured using a high-speed camera (Vision Research). 


\subsubsection{Cell Preparation}

The microfluidic channel was incubated for at least 2 hours with fibronectin (Sigma) solution at a concentration of $100 \mu \mathrm{g} \mathrm{mL}^{-1}$ to facilitate the adhesion of cells to the glass surface. After the cells were seeded into the microfluidic channel, it was incubated at $37{ }^{\circ} \mathrm{C}$ for at least two days before the experiments were conducted. The cell culture medium is composed of RPMI 1640 (Wisent Bioproducts) growth medium supplemented with $10 \%(\mathrm{v} / \mathrm{v})$ fetal bovine serum (Wisent Bioproducts) and 1\% (v/v) PenStrep (Wisent Bioproducts). For the cellular membrane staining, WGA Alexa Fluor 633 Conjugate (Thermofisher) was used at a dilution of 1:100 in PBS, and nuclear staining was carried out with Hoechst 33342 (Thermofisher) at a concentration of $0.1 \mu \mathrm{g}$ $\mathrm{mL}^{-1}$. Cells were incubated at $37^{\circ} \mathrm{C}$ for 15 minutes before images were taken.

\subsubsection{Dextran Solution Preparation}

All dextran solutions were prepared by mixing dextran in the cell culture medium. $3 \mathrm{kDa}$ Alexa 488 dextran (Thermofisher) was used at a final concentration of $50 \mu \mathrm{M} .^{275} 70 \mathrm{kDa}$ anionic FITC-dextran (Thermofisher) and $70 \mathrm{kDa}$ zwitterionic Texas Red (Thermofisher) with equal final concentrations of $0.11 \mathrm{mg} \mathrm{mL}^{-1}$ were used. ${ }^{276}$ pHrodo Green $10 \mathrm{kDa}$ dextran (Thermofisher) was used at a final concentration of $100 \mu \mathrm{g} \mathrm{mL}^{-1} .500 \mathrm{kDa}$ Fluorescein dextran (Thermofisher) with a final concentration of $10 \mu \mathrm{g} \mathrm{mL}^{-1}$ was used.

\subsubsection{Transfection Protocol}

Transfection of MDA-MB-231 cells with siRNA was carried out with a lipofectamine-based transfection by using RNAiMAX reagent (Invitrogen). The amount of lipofectamine was optimized for the GAPDH siRNA-Cy3 (Thermo Fischer Scientific) to be delivered to the cells inside the microfluidic channel. Briefly, $5.5 \mu \mathrm{l}$ of $20 \mu \mathrm{M}$ siRNA was added to $200 \mu \mathrm{l}$ of OPTIMEM, and $6.25 \mu \mathrm{l}$ of RNAiMAX was added to this mixture. This complex was added to the MDA-MB-231 cells, and images were taken 20 minutes later. Empty vector plasmid EGFP (pEGFP-C1 (Clontech 6084-1)) was used for the plasmid delivery experiments. Lipofectamine transfection was carried with Lipofectamine 3000 Reagent (Invitrogen) by optimizing the volume needed for the cells inside the microfluidic channel. Briefly, $7.4 \mu \mathrm{l}$ of Lipofectamine 3000 was 
added to $250 \mu \mathrm{l}$ of OPTIMEM in a microfuge tube. In another tube, $3 \mu 1$ of pEGFP-C1 (3.3 $\mu \mathrm{g} \mu \mathrm{l}^{-}$ ${ }^{1}$ ) and $10 \mu \mathrm{l}$ of P3000 reagent were added to $250 \mu \mathrm{l}$ of OPTIMEM. The two OPTIMEM mixtures were then mixed, incubated for 10 minutes at room temperature, and then added to the MDA-MB231 cells. The fluorescent images were taken within an hour post-treatment.

\subsubsection{Calcium Ion Measurements}

For the measurement of intracellular concentration of $\mathrm{Ca}^{+2}$, Fluo-4, AM (Thermofisher) at a final concentration of $10 \mu \mathrm{M}$ in cell culture medium was prepared. Next, cells were incubated with this solution for $20 \mathrm{~min}$. Then, cells were washed and incubated for another $20 \mathrm{~min}$, before the fluorescent images are taken. 


\section{Conclusions and Future Work}

\subsection{Summary of the Research Findings}

In this thesis, a) novel microfluidic approaches for the generation of monodisperse microbubbles for ultrasound applications and acoustofluidic strategies for b) intracellular delivery are presented. To eliminate the costly and complex microfabrication techniques needed for the production of micro- and nanobubbles, two microfluidic approaches are developed. The bubble shrinkage and breakup techniques reported in this thesis can be employed for obtaining bubble populations of the desired size. The results also show that in a flow carrying bubbles, for a given initial bubble radius, the effect of liquid surface tension and dilatational surface elasticity of the bubble on the shrinkage rate is negligible. In such a flow, bubbles can grow, shrink, or maintain their size depending on the shell permeability, liquid diffusion coefficient, liquid level of saturation, and the external pressure imposed through the side channels. By applying vacuum pressure, the final size of bubbles can be controlled, and thus this technique offers a sub-micron bubble generation methodology. In this technique, one can predict the amount of vacuum pressure required for a specific type of lipid solution in order for the collection of bubbles with the desired size.

The potential application of the bubble shrinkage technique for the production of nanobubbles is also demonstrated. A mixture of low aqueous phase solubility and high aqueous phase solubility gasses are chosen for the bubble gas core. The final size of the nanobubbles is governed by the bubble's initial size and the mixing ratio of the two gasses. These findings can be used for the development of contrast-enhanced ultrasound and targeted drug delivery applications. 
Moreover, an expansion-mediated breakup of bubbles (and droplets) is also presented. By controlling the governing dimensionless numbers, one can predict the size distribution of the outlet population being mono-, bi-, or tri-disperse. The modeling framework and the dimensional analysis presented here for the prediction of the bubble size can also be useful for designing future droplet-based lab-on-a-chip devices.

A novel acoustofluidic strategy is reported for developing cytosolic delivery - which could also be used for characterizing the elastic properties of cells. The excitation of adherent cells using acoustic waves in a microfluidic channel enables the cellular stiffness measurement and facilitates cellular uptake. Due to the acoustic actuation of cells, microstreaming flow is generated surrounding the cell inside the acoustofluidic device. The velocity of microstreaming flow induced by a cell is shown to be directly related to the overall Young's modulus of the cell. Cellular stiffness, as a biomarker of cancer cell function, adherence, motility, transformation, and invasion, ${ }^{277,278}$ can, therefore, be probed in a high-throughput fashion using this acoustofluidic platform.

A prolonged actuation of cells at relatively high acoustic pressures exhibits an enhanced cellular uptake as a result of the combined effect of acoustic excitation and microstreaming flow. The successful delivery of cargo materials with different sizes and charges into various cell lines is demonstrated. Due to the high cellular viability, actuation controllability, operation scalability and portability of this acoustofluidic strategy, it can be useful for a variety of in vitro cancer biology and drug-screening applications.

\subsection{Future Work}

The research presented in this thesis can be further explored in the directions outlined below.

A change to the composition of the bubble shell can impact its lifetime, and thus, low interfacial tension and low shell permeability can lead to a longer bubble lifetime. The production of microbubbles with various shell compositions can be studied in order to identify the effect of shell properties on the lifetime of the shrunk bubbles. One important question related to the bubble 
lifetime is: what happens to the shell structure when the bubble size changes due to shrinkage? Does the shell retain its structure, or does shell buckling occur? The shell structure, at the molecular level, of sub-micron bubbles, can be of great importance, as it can explain the acoustic response and high stability of nanobubbles.

The approach reported in Chapters 2 and 3 for obtaining controlled shrinkage and breakup of bubbles can also be useful for a variety of bubble-based applications, including the fabrication of biomaterials (e.g., scaffolds, porous thin films, and protein bubbles), ${ }^{279}$ gas-liquid microreactor development, ${ }^{176,280,281}$ and DNA origami studies. ${ }^{282}$ In conventional bubble-generator systems, the size of bubbles is not controlled after the bubbles are generated. If a pressure control unit coupled with a permeable microfluidic device is used, an additional control parameter will be provided for tuning the final pore size in biomaterial production technologies or the reaction kinetics in microreactors.

The possibility of obtaining a stepwise breakup of bubbles (or droplets) inside a microfluidic device, which features multiple expansion-mediated regions with channels in a branched configuration, can also be tested. In such a multi-step bubble breakup device, in addition to the dimensionless numbers discussed in Chapter 3, the number of expansion regions that each bubble flows through can also impact the final size distribution of bubbles meaning that a mono-disperse or multi-disperse population can be achieved.

Using the acoustofluidic platform developed in Chapters 5 and 6, the cell resonant frequency and its dependence on the cell shape and morphology can also be studied. The physical and biological responses of cells to oscillations at their resonant frequencies is another interesting research direction. Since at a resonant frequency, compared to non-resonant frequencies, the maximum oscillation amplitude is higher, a lower level of acoustic energy will theoretically be required for obtaining equal oscillation amplitudes. This could help to avoid the transducer heating up while achieving the maximum acoustic excitation effect.

The acoustofluidic strategy developed for cellular elastography can be further calibrated using a gold-standard technique, such as atomic force microscopy. A high-throughput device for measuring Young's modulus can be fabricated and tested across various cell lines. As an example, 
this technique may enable a quick, non-invasive, and cost-effective methodology for differentiating between healthy and cancerous cells. ${ }^{240,283}$

A high-throughput acoustofluidic device containing numerous regions of almost uniform acoustic pressures can be fabricated and tested for the intracellular delivery across various hardto-transfect cell lines and stem cells. Hundreds of thousands of cells adhered to these regions can be treated with different excitation parameters and treatment durations. Moreover, the efficacy of the delivered genes in regulating cell functions can be further investigated using this intracellular delivery device.

Exposing cells to acoustic pressures higher than the pressure range used for intracellular delivery can force the cells to lose their adhesion to the substrate. The applicability of this acoustofluidic technique in culturing adherent cells as an enzyme-free tool for cell dissociation can be studied in the future.

The microfluidic approaches and acoustofluidic platform reported in this thesis are portable, easy-to-operate, and biocompatible, and therefore, can be further optimized for being used in labon-a-chip devices. 


\section{Appendices}

\section{Appendix A: Supporting Information for Chapter 2}

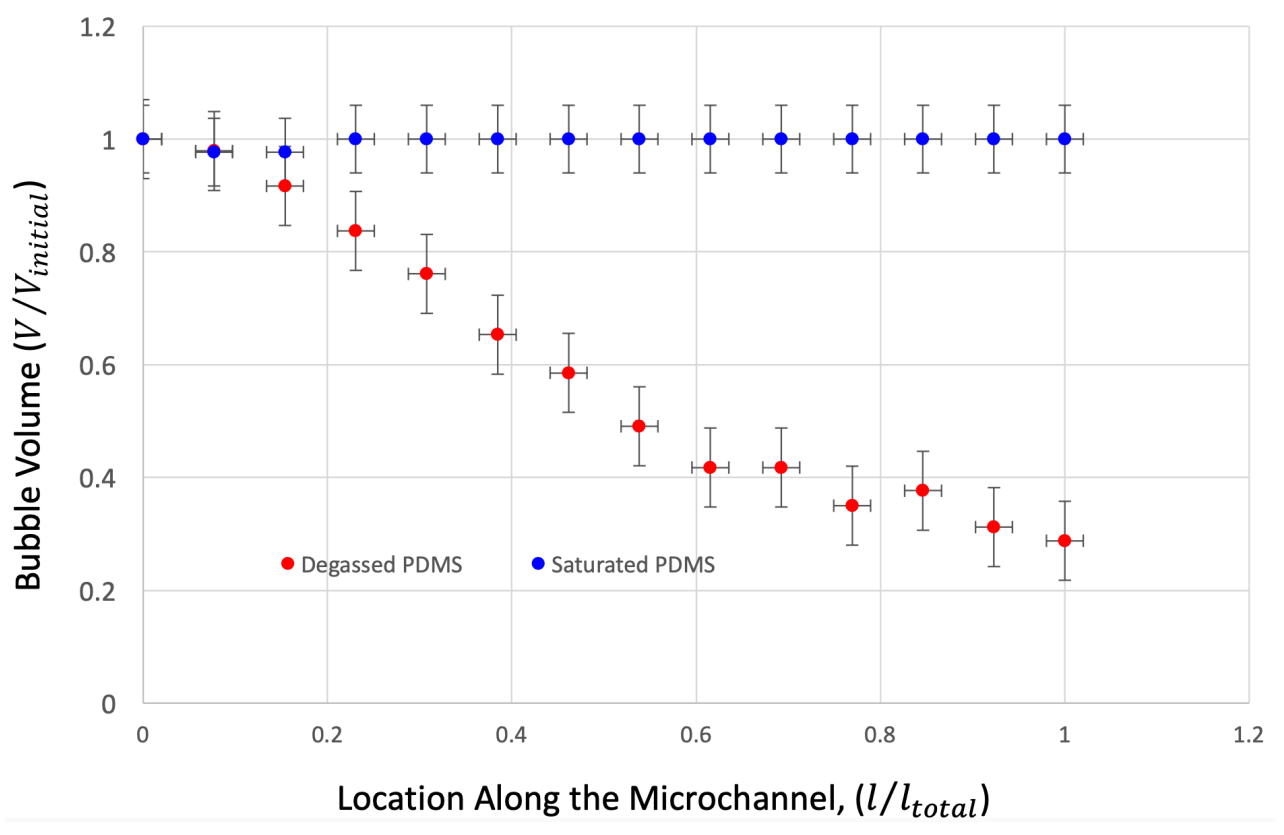

Figure A.1 Comparison of bubble shrinkage results along the microfluidic channel for a degassed PDMS device, and a saturated PDMS device left in atmospheric condition for 24 hours prior to usage. In these experiments, the aqueous liquid flow rate and air pressure are $3 \mu \mathrm{Lmin}^{-1}$ and $25.5 \pm 1.5 \mathrm{kPa}$, respectively. The vacuum pressure $P_{v}=0$. 


\section{Appendix B: Supporting Information for Chapter 3}

\section{Video B.1}

This video shows a periodic symmetric breakup of bubbles and is accessible at https://journals.aps.org/prfluids/supplemental/10.1103/PhysRevFluids.5.013602

\section{Video B.2}

This video shows a periodic asymmetric breakup of bubbles and is accessible at https://journals.aps.org/prfluids/supplemental/10.1103/PhysRevFluids.5.013602

\section{Video B.3}

This video shows a periodic symmetric breakup of oil droplets and is accessible at https://journals.aps.org/prfluids/supplemental/10.1103/PhysRevFluids.5.013602

\section{Video B.4}

This video shows a periodic asymmetric breakup of oil droplets and is accessible at https://journals.aps.org/prfluids/supplemental/10.1103/PhysRevFluids.5.013602 


\section{Appendix C: Supporting Information for Chapter 4}

Note C.1 Preparation of the lab-made gas mixture

For preparing the lab-made mixture of $\mathrm{C}_{3} \mathrm{~F}_{8}$ in air, we used a prefilled $500 \mathrm{~mL}$ graduated glass bottle which was immersed into a water sink upside down, as shown in Figure C.1.

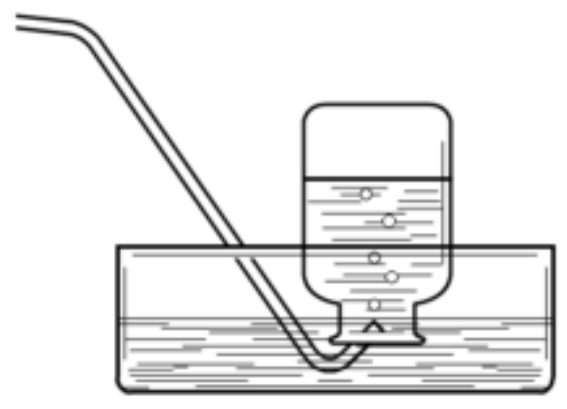

Figure C.1 A schematic of the custom-made setup for mixing gasses.

A mixture of $42 \mathrm{wt} \% \mathrm{C}_{3} \mathrm{~F}_{8}$ was prepared by injecting a $270 \mathrm{~mL}$ of air and a $30 \mathrm{~mL}$ of pure $\mathrm{C}_{3} \mathrm{~F}_{8}$ (SynQuest Labs Inc.) into the bottle, while the volume of each gas was estimated by the displacement of water at a constant pressure. The gas mixture was then extracted using a $50 \mathrm{~mL}$ syringe. A mixture of $4.2 \mathrm{wt} \% \mathrm{C}_{3} \mathrm{~F}_{8}$ was prepared by mixing a $270 \mathrm{~mL}$ of air and $30 \mathrm{~mL}$ of $42 \mathrm{wt} \%$ $\mathrm{C}_{3} \mathrm{~F}_{8}$ mixture. A gas mixture of $0.42 \mathrm{wt} \%$ was then prepared by mixing a $270 \mathrm{~mL}$ of air and a 30 $\mathrm{mL}$ of $4.2 \mathrm{wt}^{\circ} \mathrm{C}_{3} \mathrm{~F}_{8}$. 


\section{Appendix D: Supporting Information for Chapter 5}
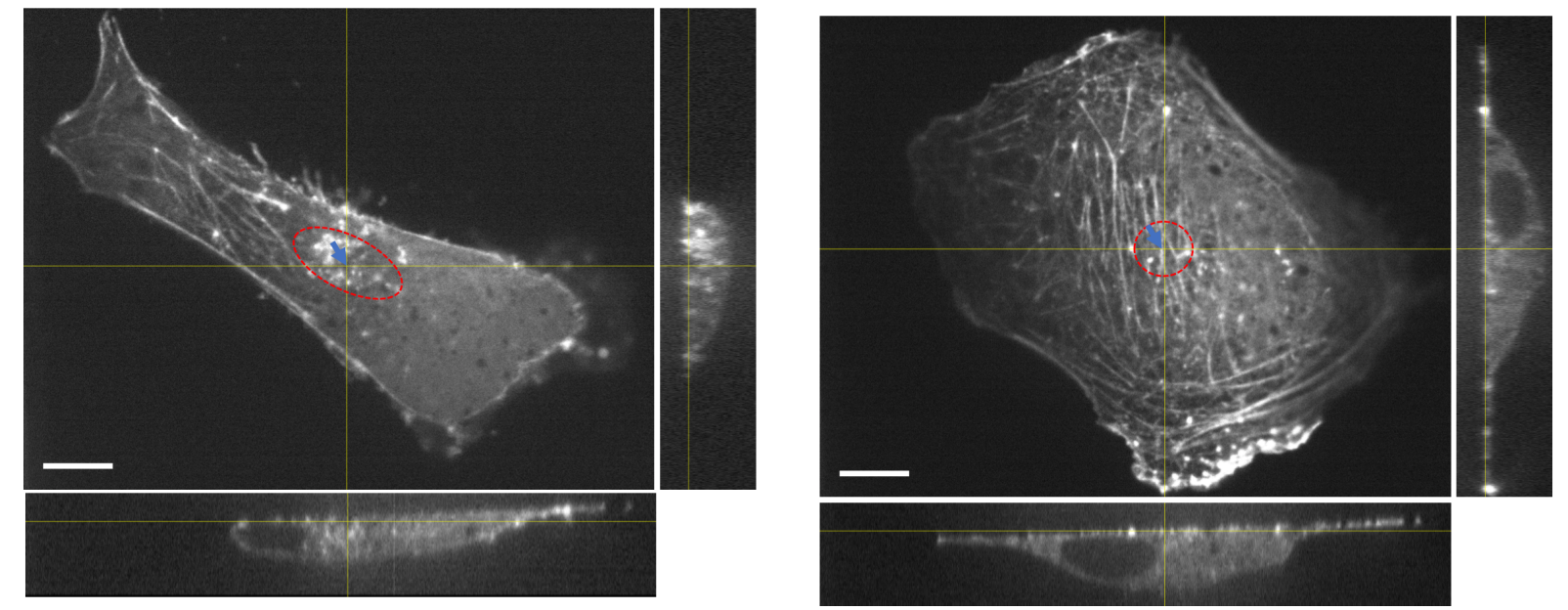

Figure D.1 Confocal images of the two MDA-MB-231 cells studied in Section 2. The cells having an equal height of $\sim 10 \mu \mathrm{m}$ are stained with Actin-GFP. On each cell, the dashed red line roughly outlines the region of the cell's maximum height. The center of this region (i.e., the point of maximum height) is marked by a blue arrow. The scale bars represent $20 \mu \mathrm{m}$. 


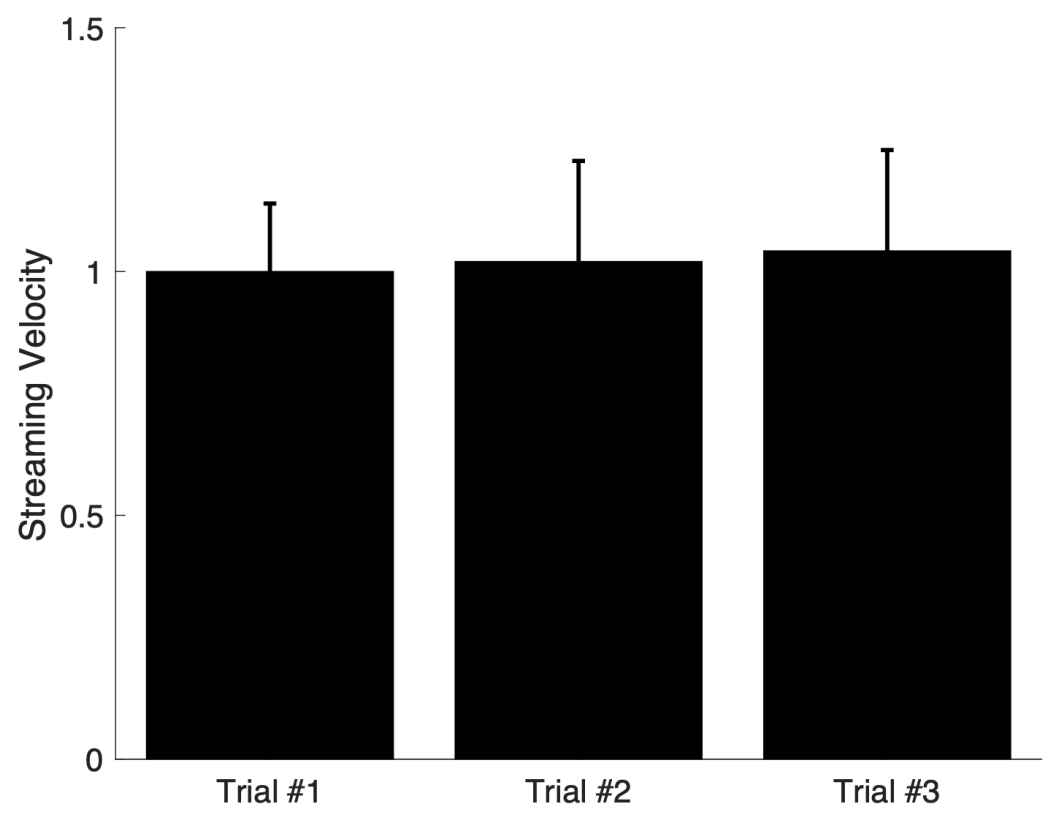

Figure D.2 The results of three different trials of maximum microstreaming velocity measurements of MDA-MB-231 cells $(n=10)$.
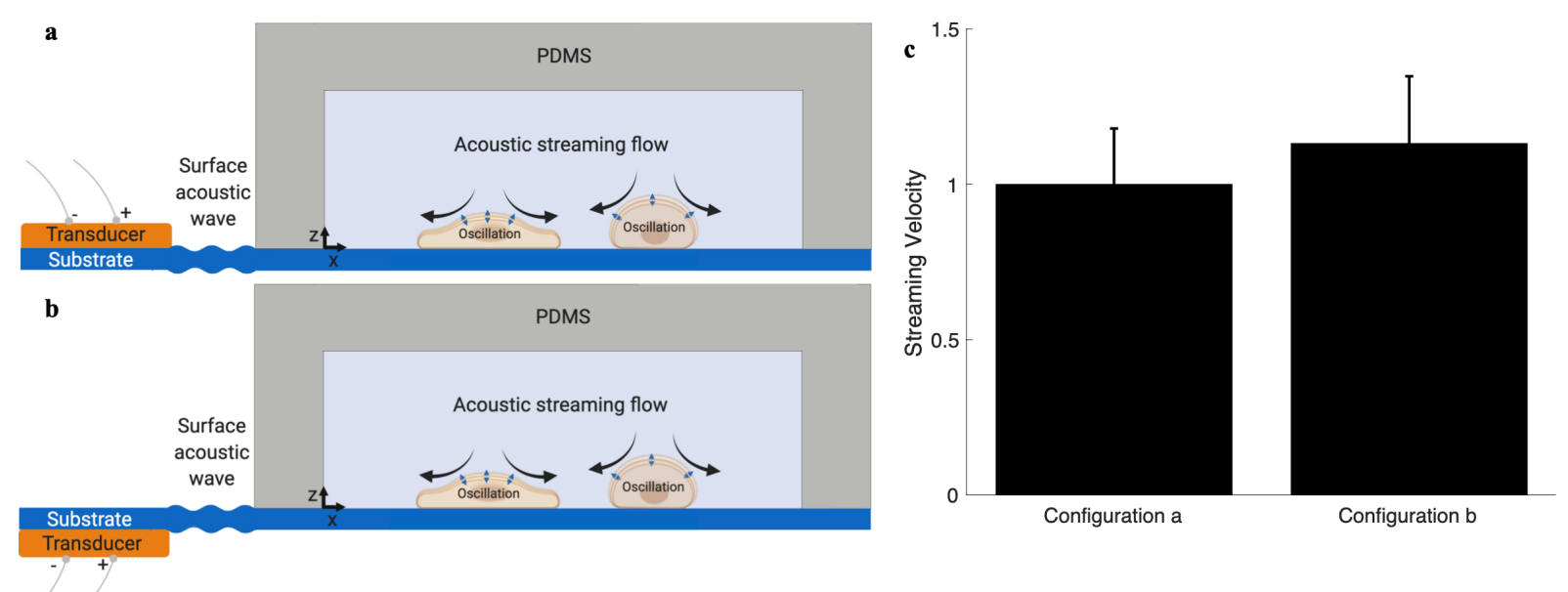

Figure D.3 (a-b) Schematic diagram (not to scale) of the two different device configurations. (a) PZT transducer and the microfluidic channel are attached onto the same surface of the substrate. (b) PZT transducer and the microfluidic channel are attached onto the opposite surfaces of the substrate. Theoretically, cells on either surface of the substrate can be excited by Lamb waves, regardless of the surface on which the acoustic waves are introduced. (c) The results of streaming flow induced by the same cells (i.e., MDA-MB-231) in these two device configurations. An acoustic field can form in the substrate regardless of the surface on which the transducer is 
attached, and thus, cells can exhibit microstreaming flow in either of the two device configurations. The actuation frequency is $98 \mathrm{kHz}$.

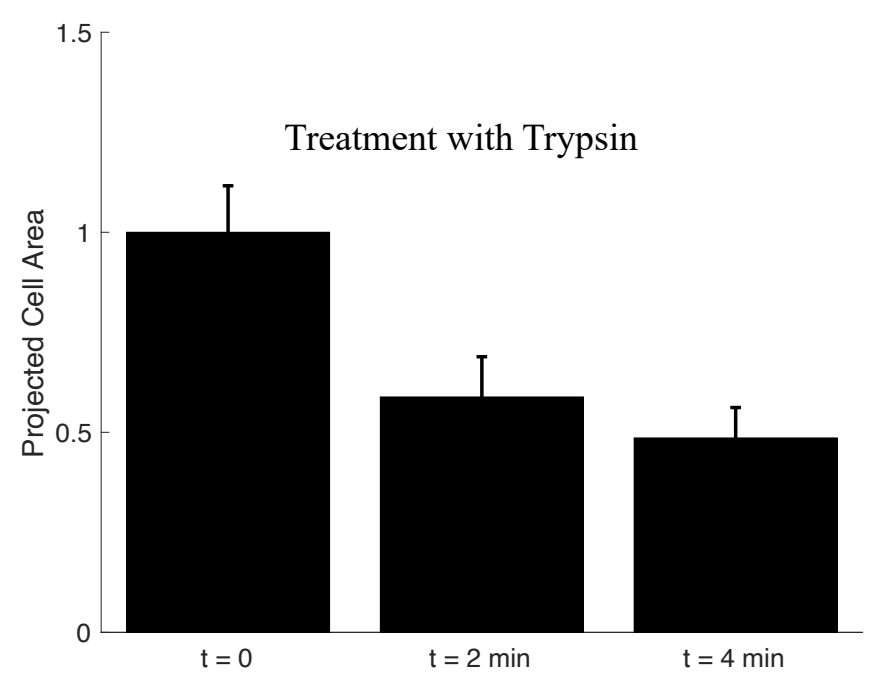

Figure D.4 Projected area measured by phase-contrast microscopy during the treatment of MDAMB-231 cells with trypsin. The error bars represent one standard deviation $(n \geq 10)$.

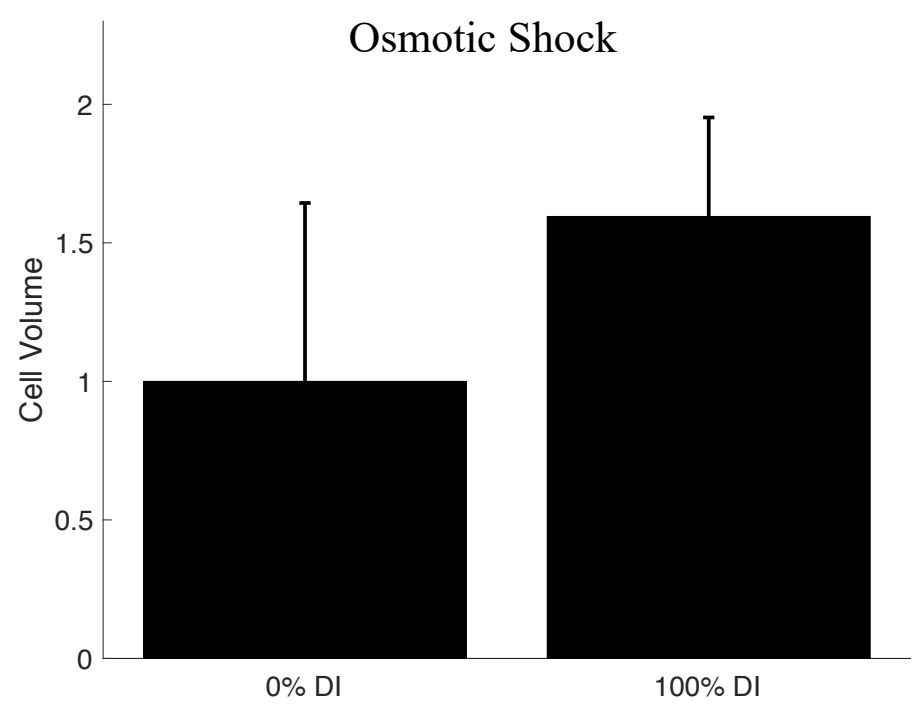

Figure D.5 Cell volume measurements before and after an osmotic shock is imposed on the MDAMB-231 cells. The cells are first stained with Actin-GFP, and then fluorescent images at equal $z$ steps are taken. The volume of each cell is estimated by adding all cross-sectional area obtained from the fluorescent images. The error bars represent one standard deviation $(n \geq 7)$. 

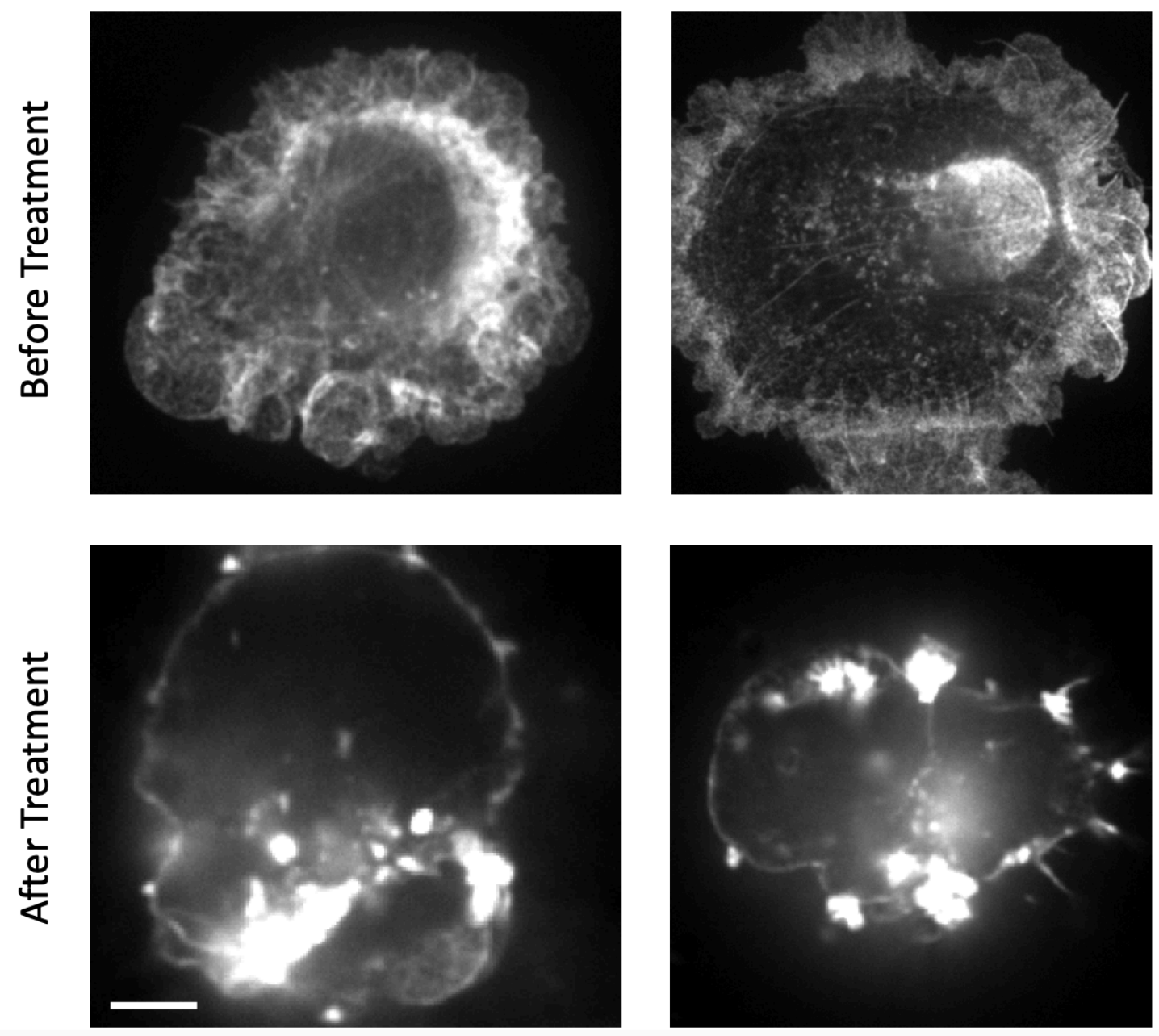

Figure D.6 MDA-MB-231 cells stained with Phalloidin showing actin filament distribution before and after treatment with $4 \mu \mathrm{M}$ Cytochalasin D. As shown, actin filaments are depolymerized after the treatment and coagulated randomly in localized regions, as opposed to the untreated cells, where, the cells exhibit a more uniform distribution of actin filaments throughout their structure. The scale bar represents $20 \mu \mathrm{m}$. 


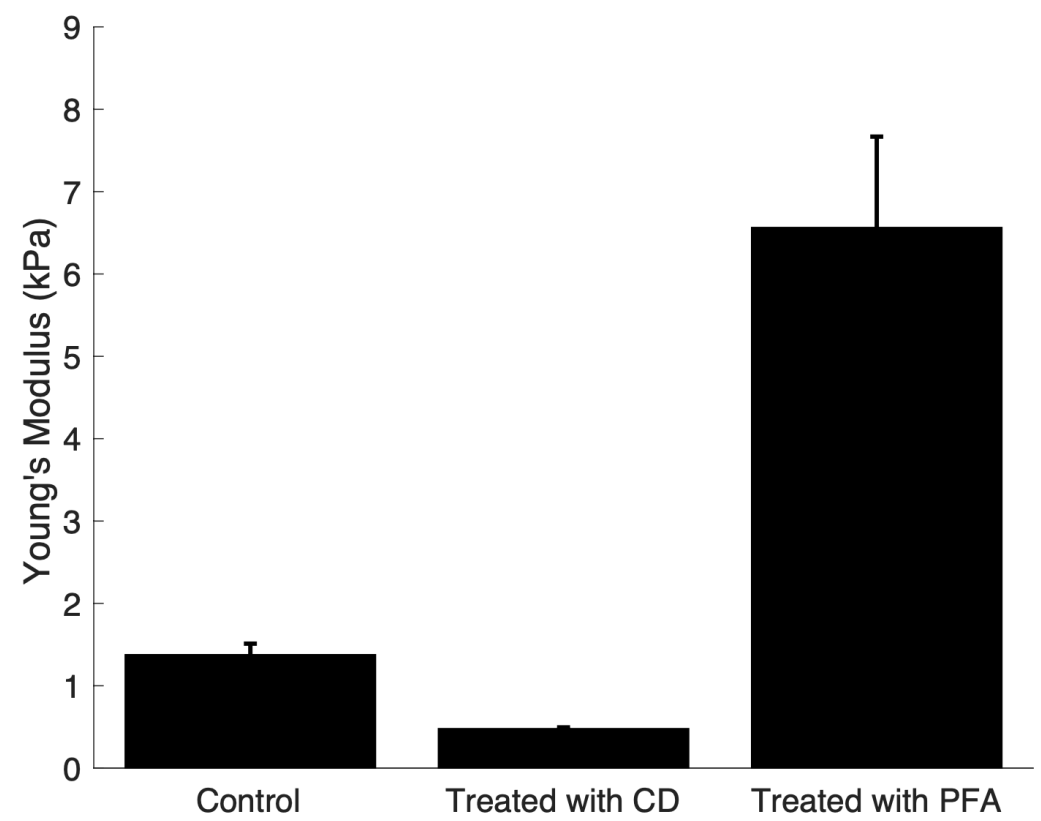

Figure D.7 Young's elastic modulus measurements using AFM. Live (control) cells exhibit an average Young's modulus of $1.38 \mathrm{kPa}$. Treatment with Cytochalasin D results in a $\sim$ three-fold lower average Young's modulus of $0.48 \mathrm{kPa}$, whereas those chemically fixed with PFA are stiffer than controls with a $\sim$ five-fold higher average Young's modulus of $6.57 \mathrm{kPa}$. The error bars represent one standard deviation of all processed force measurements ( $\sim 500$ per cell) of four cells per group. 


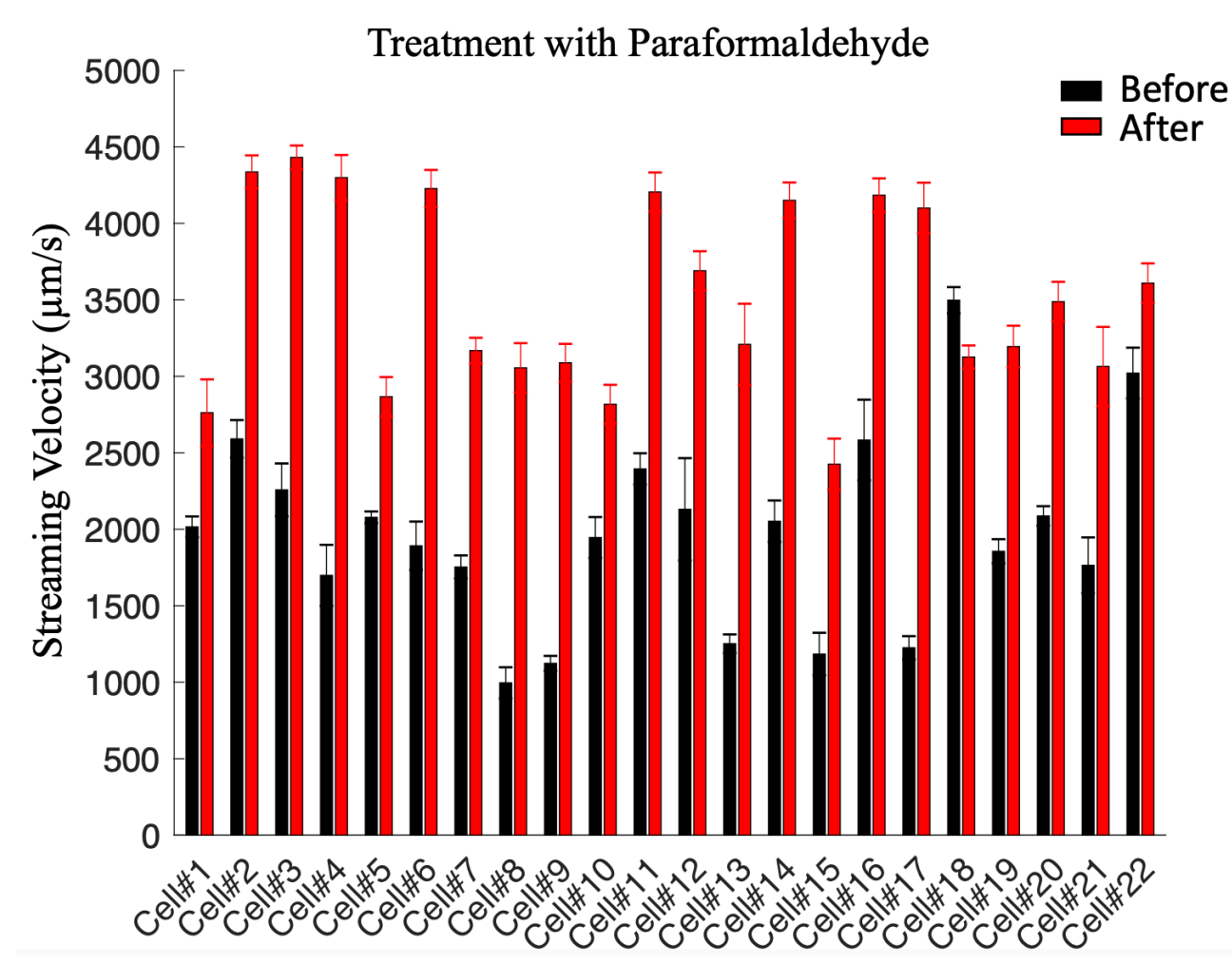

Figure D.8 The maximum streaming velocity induced by individual MDA-MB-231 cells monitored before and after treatment with PFA solution. The error bars represent one standard deviation.

Note D.1 Numerical simulation

In order to model the cell-induced microstreaming flow, we conduct a perturbation approximation using thermoviscous acoustics and laminar flow physics. ${ }^{85,284,285}$ Briefly, first we design 3D microfluidic domains of $300 \times 300 \times 28 \mu \mathrm{m}$ containing single-cells with a diameter of 30 $\mu \mathrm{m}$ for the round cell and $36 \mu \mathrm{m}$ for the flat cell adhered to the bottom surfaces. Then, $1^{\text {st }}$ order acoustic field is solved for the entire domain assuming a harmonic boundary condition with a 10 $\mathrm{nm}$ amplitude in all three directions (i.e., one normal and two tangents) imposed on the cell membrane. The $1^{\text {st }}$ order equations are as follows: 284,285 


$$
\begin{gathered}
\frac{\partial \rho_{1}}{\partial t}+\rho_{0}\left(\nabla \cdot \mathbf{v}_{1}\right)=0 \\
\rho_{0} \frac{\partial \mathbf{v}_{1}}{\partial t}=-\nabla p_{1}+\mu \nabla^{2} \mathbf{v}_{1}+\beta \mu \nabla\left(\nabla \cdot \mathbf{v}_{1}\right) \\
\frac{\partial T_{1}}{\partial t}=\frac{k}{\rho_{0} C_{p}} \nabla^{2} T_{1}+\frac{\alpha T_{0}}{\rho_{0} C_{p}} \frac{\partial p_{1}}{\partial t}
\end{gathered}
$$

where, $\rho, \mathbf{v}, p, \mu, \beta, T, k, C_{p}$, and $\alpha$ are mass density, fluid velocity, fluid pressure, fluid viscosity, viscosity ratio, fluid temperature, thermal conductivity of the fluid, specific heat capacity at constant pressure, and thermal expansion coefficient, respectively.

The streaming flow is the solution to the laminar flow physics assuming the time-averaged values of the $1^{\text {st }}$ order quantities added as mass source and volume forces to the laminar flow equations:

$$
\begin{gathered}
\left\langle\frac{\partial \rho_{2}}{\partial t}\right\rangle+\rho_{0}\left(\nabla \cdot\left\langle\mathbf{v}_{2}\right\rangle\right)=-\nabla \cdot\left\langle\rho_{1} \mathbf{v}_{1}\right\rangle \\
\rho_{0}\left\langle\frac{\partial \mathbf{v}_{2}}{\partial t}\right\rangle=-\nabla\left\langle p_{2}\right\rangle+\mu \nabla^{2}\left\langle\mathbf{v}_{2}\right\rangle+\beta \mu \nabla\left(\nabla .\left\langle\mathbf{v}_{2}\right\rangle\right)-\left\langle\rho_{1} \frac{\partial \mathbf{v}_{1}}{\partial t}\right\rangle-\rho_{0}\left\langle\left(\nabla \cdot \mathbf{v}_{1}\right) \mathbf{v}_{1}\right\rangle
\end{gathered}
$$

Once $\mathbf{v}_{2}$ is obtained, we generate the velocity field and plot the streaming flow lines. 
We use COMSOL Multiphysics to solve above equations. A physics-controlled mesh structure for the entire fluidic domain with an average element size of $\sim 4 \mu \mathrm{m}$ is used. Table D.1 summarizes the property values of the fluidic domain (assuming as water) used in the simulations.

Table D.1 The property values used in the numerical simulation

$\begin{array}{cl}\text { Property } & \text { Value } \\ f & 98 \mathrm{kHz} \\ \rho & 1000 \mathrm{~kg} \mathrm{~m}^{-3} \\ \mu & 8.5 \times 10^{-4} \mathrm{~Pa} \mathrm{~s} \\ \beta & 0.33 \\ k & 0.61 \mathrm{~W}(\mathrm{~m} \mathrm{~K})^{-1} \\ C_{p} & 4180 \mathrm{~J}\left(\mathrm{~kg} \mathrm{~K}^{-1}\right. \\ \alpha & 2.75 \times 10^{-4} \mathrm{~K}^{-1}\end{array}$

Note D.2 Microstreaming flow measurement

We take high-speed videos for $4 \mathrm{~s}$ capturing the beads with the maximum speed surrounding the cells. The video frames are then extracted for further analysis. We use a semi-automated singleparticle tracking technique, which is also available as an ImageJ plugin, called TrackMate. ${ }^{224}$ Briefly, first we specify the estimated bead diameter per pixel, and after adjusting a threshold, beads are detected in all frames using a Laplacian of Gaussian filter. Next, we conduct the bead tracking step by adjusting the beads' maximum travel distance (in pixels) across every two successive frames. It should be noted that bead tracks obtained at the end of this step have different lengths depending on the number of frames the beads appear on the focal plane. We, therefore, keep the more reliable tracks, i.e., those on the top $\sim 10 \%$ of the track displacement list. We then take the average velocity (pixel per frame) of the beads in the remaining tracks (Figure D.9). 10 tracks with the highest average velocity are taken as the streaming flow measurements for each cell. Measurements from all cells are then combined, averaged, and their standard deviations are calculated. 

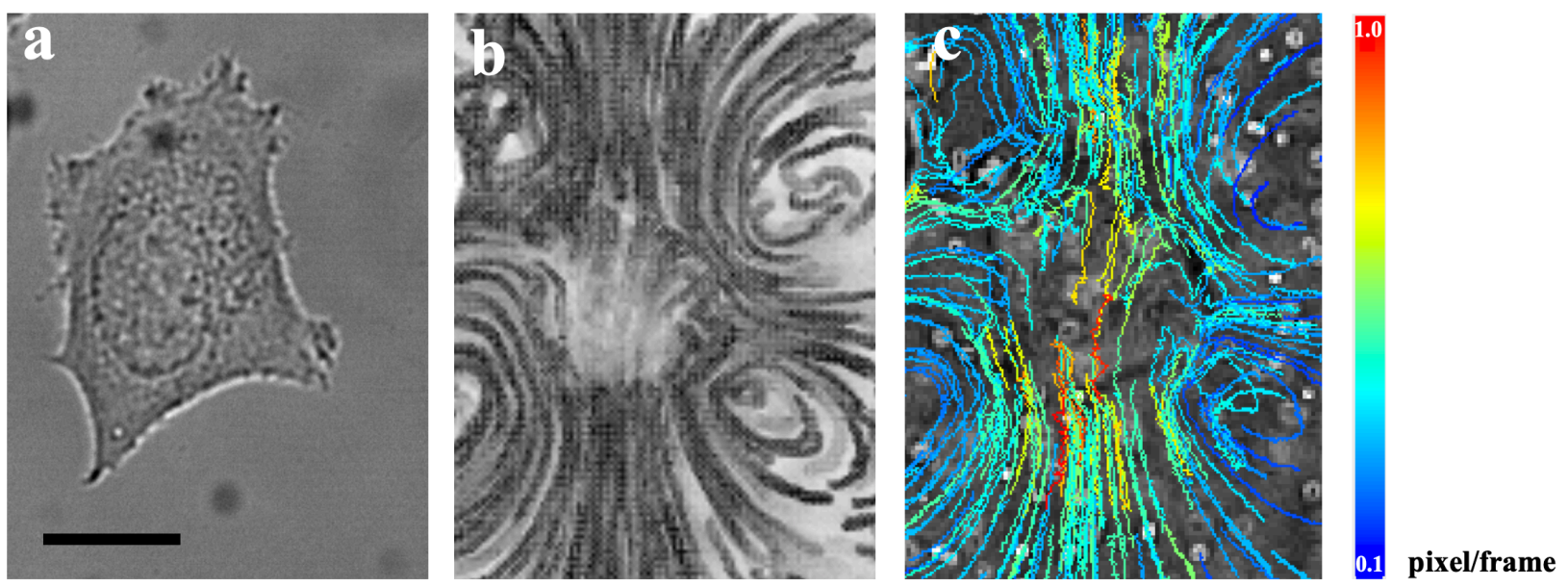

Figure D.9 (a) Bright-field image of an MDA-MB-231 cell. (b) Stacked image showing the tracks of $1 \mu \mathrm{m}$ beads in the liquid once the cell is driven at $98 \mathrm{kHz}$. (c) The output of the semi-automated single-particle tracking technique showing the bead tracks. The flow patterns detected by the automated technique match those found by the stacked image. The color bar represents the mean velocity (in pixel per frame). The scale bar represents $20 \mu \mathrm{m}$.

\section{Video D.1}

This video shows an example of cell-induced flow generated when the cells are driven at 98 $\mathrm{kHz}$. 1 um beads are mixed with the fluid. It is accessible at https://onlinelibrary.wiley.com/action/downloadSupplement?doi=10.1002\%2Fsmll.201903788\& $\underline{\text { file }=\text { smll201903788-sup-0002-VideoS1.mov }}$

\section{Video D.2}

This video shows an acoustically generated fluid flow inside the microfluidic channel at 98 $\mathrm{kHz}$ when no cells are present. 1um beads are mixed with the fluid. It is accessible at https://onlinelibrary.wiley.com/action/downloadSupplement?doi=10.1002\%2Fsmll.201903788\& $\underline{\text { file }=\text { smll201903788-sup-0003-VideoS2.mov }}$ 


\section{Video D.3}

This video shows fluorescent tracers following the microstreaming flow induced by an MCF-7 cell at a height of $z=7 \mu \mathrm{m}$ above the substrate surface. The actuation frequency is 98 $\mathrm{kHz}$. It is accessible at https://onlinelibrary.wiley.com/action/downloadSupplement?doi=10.1002\%2Fsmll.201903788\& $\underline{\text { file }=\text { smll201903788-sup-0004-VideoS3.mov }}$

\section{Video D.4}

This video shows fluorescent tracers following the microstreaming flow induced by an MCF-7 cell at a height of $z=14 \mu \mathrm{m}$ above the substrate surface. The actuation frequency is 98 $\mathrm{kHz}$. It is accessible at https://onlinelibrary.wiley.com/action/downloadSupplement?doi=10.1002\%2Fsm11.201903788\&

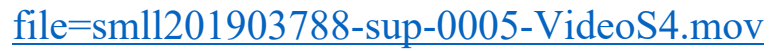

\section{Video D.5}

This video shows fluorescent tracers following the microstreaming flow induced by an MCF-7 cell at a height of $z=21 \mu \mathrm{m}$ above the substrate surface. The actuation frequency is 98 $\mathrm{kHz}$. It is accessible at https://onlinelibrary.wiley.com/action/downloadSupplement?doi=10.1002\%2Fsm11.201903788\& $\underline{\text { file }=\text { smll201903788-sup-0006-VideoS5.mov }}$

\section{Video D.6}

This video shows the microstreaming flow induced by an MDA-MB-231 cell at an actuation frequency of $32 \mathrm{kHz} .1 \mu \mathrm{m}$ beads are mixed with the fluid. It is accessible at https://onlinelibrary.wiley.com/action/downloadSupplement?doi=10.1002\%2Fsm11.201903788\& $\underline{\text { file }=\text { smll201903788-sup-0007-VideoS6.mov }}$ 


\section{Video D.7}

This video shows the microstreaming flow induced by an MDA-MB-231 cell at an actuation frequency of $73 \mathrm{kHz} .1 \mu \mathrm{m}$ beads are mixed with the fluid. It is accessible at https://onlinelibrary.wiley.com/action/downloadSupplement?doi=10.1002\%2Fsmll.201903788\& $\underline{\text { file }=\text { smll201903788-sup-0008-VideoS7.mov }}$

\section{Video D.8}

This video shows the microstreaming flow induced by an MDA-MB-231 cell at an actuation frequency of $98 \mathrm{kHz} .1 \mu \mathrm{m}$ beads are mixed with the fluid. It is accessible at https://onlinelibrary.wiley.com/action/downloadSupplement?doi=10.1002\%2Fsmll.201903788\& $\underline{\text { file }=\text { smll201903788-sup-0009-VideoS8.mov }}$

\section{Video D.9}

This video shows the effect of MDA-MB-231 cell oscillation on a $5 \mu \mathrm{m}$ bead when the cell is actuated at a frequency of $32 \mathrm{kHz}$. It is accessible at https://onlinelibrary.wiley.com/action/downloadSupplement?doi=10.1002\%2Fsmll.201903788\& $\underline{\text { file }=\text { smll201903788-sup-00010-VideoS9.mov }}$

\section{Video D.10}

This video shows the effect of MDA-MB-231 cell oscillation on a $10 \mu \mathrm{m}$ bead when the cell is actuated at a frequency of $32 \mathrm{kHz}$. It is accessible at https://onlinelibrary.wiley.com/action/downloadSupplement?doi=10.1002\%2Fsmll.201903788\& $\underline{\text { file }=\text { smll201903788-sup-00011-VideoS10.mov }}$

\section{Video D.11}

This video shows a motion of intracellular vesicles caused by the oscillation of an MDAMB-231 cell membrane. The actuation frequency is $98 \mathrm{kHz}$. It is accessible at 
https://onlinelibrary.wiley.com/action/downloadSupplement?doi=10.1002\%2Fsmll.201903788\&

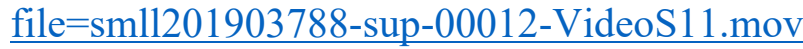




\section{Appendix E: Supporting Information for Chapter 6}

Note E.1 Image analysis protocol

To quantify the uptake, the fluorescence images of control and treated cells were taken using consistent levels of illumination and excitation-emission filters of 480/17-517/23 nm for green fluorescence imaging and 556/20-615/61 nm for red fluorescence imaging. For each experiment, we took the bright-field and fluorescent images of the regions of interest (Figure E.1a-b). Here, we assume the amount of delivery is directly proportional to the fluorescence intensity of the cargo material. ${ }^{286}$ To quantify the uptake, we use a semi-automated protocol, which consisted of five steps, as follows.

- Step \#1: First, we use ImageJ to select the areas covered by the cells on the brightfield image (Figure E.1c).

- Step \#2: Then, the selected area is copied to the fluorescent image (Figure E.1d).

- Step \#3: Next, the average fluorescence intensity of the selected area from the fluorescent image is calculated

(i.e., $I_{1}=\frac{\text { Sum of the values of the pixels of the selected area }}{\text { Selected area }}$ ).

- Step \#4: Then, the average fluorescence intensity of the regions outside the selected area is calculated (i.e., $I_{2}=\frac{\text { Sum of the values of the pixels outside the selection }}{\text { Area outside the selection }}$ ).

- Step \#5: The final step is to calculate the difference of $I_{l}$ and $I_{2}$, which is considered as the average intensity of the delivered material to the cells (i.e., Mean Intensity = $\left.I_{1}-I_{2}\right)$. 

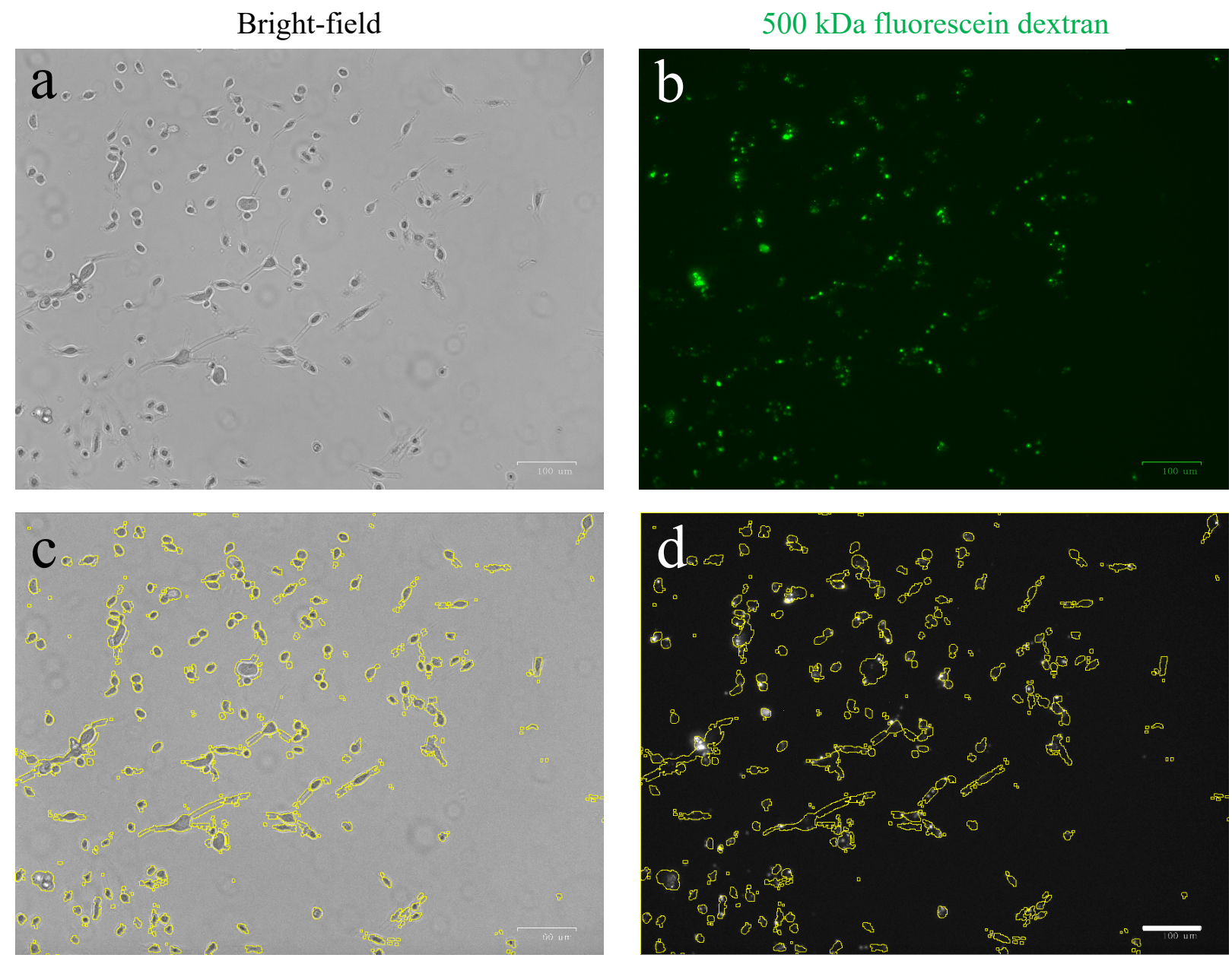

Figure E.1 Sample (a) bright-field and (b) fluorescent images taken from the regions of interest. (c) The outline of the cells on the bright-field image is selected using ImageJ. (d) The selected area is then transferred to the fluorescent image for further intensity calculations. The scale bar represents $100 \mu \mathrm{m}$.

To quantify the delivery efficiency, we take the control experiments as the reference for finding a fluorescence intensity threshold $\left(I_{t}\right)$. This threshold is defined as an intensity that is higher than the mean intensity of $95 \%$ of the cell population in the control experiments. ${ }^{42,43}$ In other words, only $5 \%$ of control cells exhibit fluorescence intensities higher than $I_{t}$. The delivery efficiency of each treatment is calculated as follows: 
Delivery efficiency $(\%)=\frac{\text { Number of treated cells having a mean intensity above } I_{t}}{\text { Total number of treated cells }} \times 100$

a

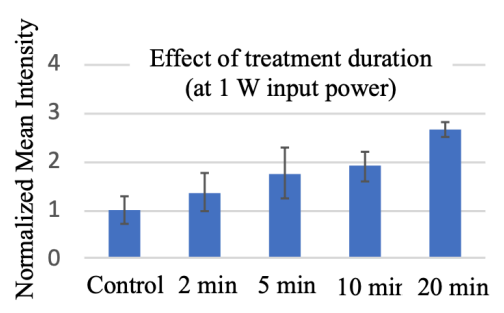

b

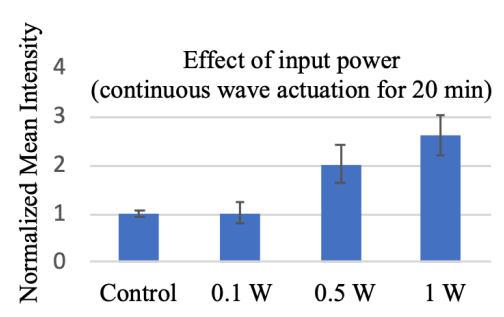

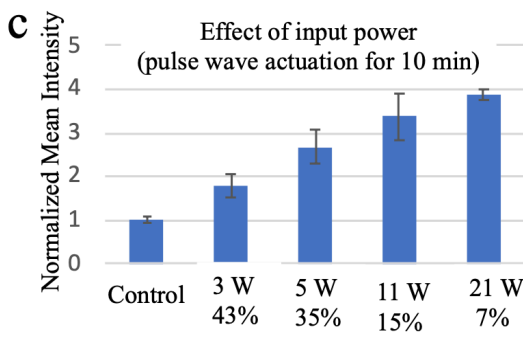

Figure E.2 The effect of input power and treatment duration on intracellular delivery. (a) The delivery results for different treatment duration when continuous $1 \mathrm{~W}$ input power is applied. (b) Delivery results for the input power in the range of $0.1-1 \mathrm{~W}$ and 20 min treatment. (c) Delivery results for different input powers in the range of 3-21 W. For each input power, the duty cycle of the square-waves is set in the range of $7-43 \%$ for $10 \mathrm{~min}$ treatment duration, to avoid excessive heat generated by the transducer and vibrating PDMS. In all experiments, $3 \mathrm{kDa}$ dextran is used as a cargo material for delivery into MDA-MB-231 cells. The mean intensities are normalized by the values of control experiments. The error bars indicate standard deviation of three independent experiments. 

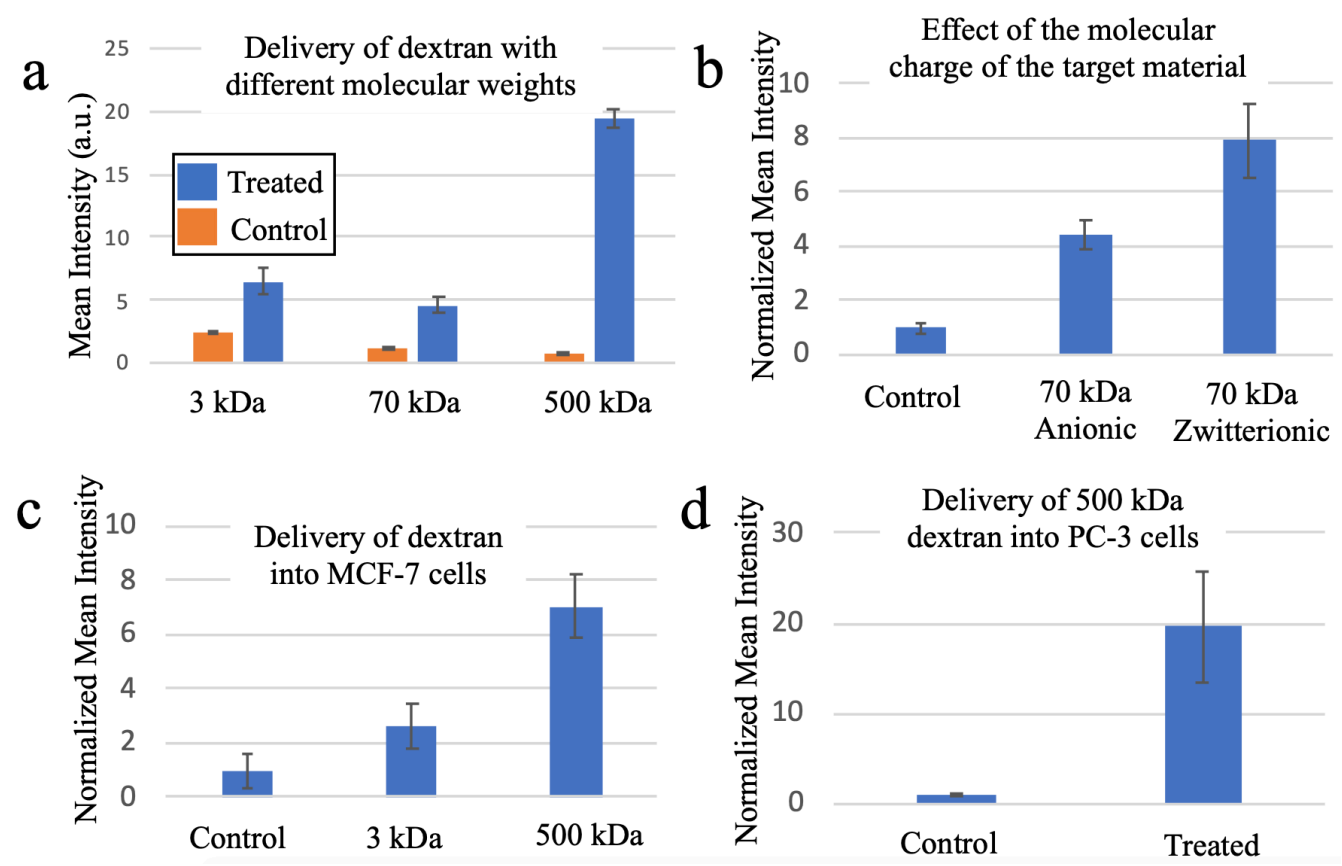

Figure E.3 The versatility of intracellular delivery of dextran with various sizes and charges into different cells. Delivery results for dextran (a) with different molecular weights and (b) charges into MDA-MB-231 cells. Delivery results for (c) MCF-7 and (d) PC3 cells. A 20 min continuous square-wave at $1 \mathrm{~W}$ input power is used in all experiments. The mean intensities in (b-d) are normalized by the values of control experiments. The error bars indicate standard deviation of three independent experiments. 
a
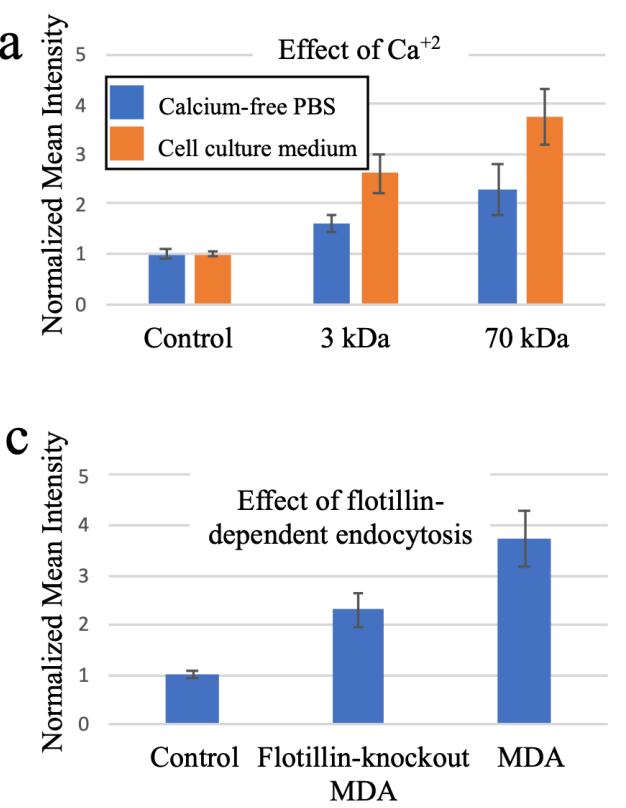

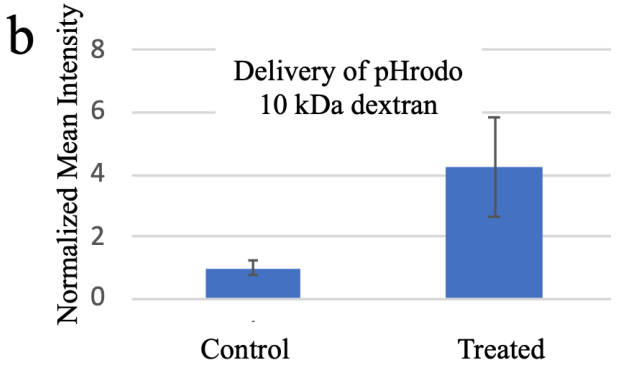

d

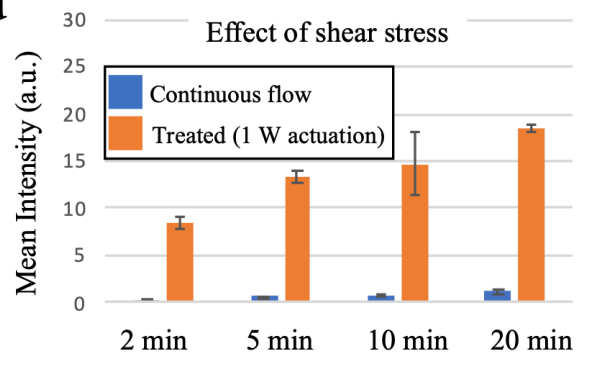

Figure E.4 Probing possible mechanisms of delivery. (a) Effect of $\mathrm{Ca}^{+2}$ on the delivery of $3 \mathrm{kDa}$ and $70 \mathrm{kDa}$ dextran. (b) Delivery results of pHrodo Green $10 \mathrm{kDa}$ dextran, which fluoresces at low $\mathrm{pH}$ values. In these experiments, the fluorescence images of the treated and control cells are taken while the cells are exposed to the solution containing pHrodo dextran. Therefore, the delivery of pHrodo Green $10 \mathrm{kDa}$ dextran is an indication of the endosomal pathway role in intracellular delivery. (c) Effect of flotillin-dependent endocytosis on the amount of delivery. (d) Exposure to continuous flow (shear stress only) and acoustic excitation on the amount of delivery for different exposure durations. Acoustically actuated cells exhibit significantly higher uptake compared to those exposed to shear stress only. Treatments are conducted on MDA-MB-231 cells using a 20 min continuous square-wave at $1 \mathrm{~W}$ input power. The mean intensities in $(\mathrm{a}-\mathrm{c})$ are normalized by the values of control experiments. The error bars indicate standard deviation of three independent experiments. 

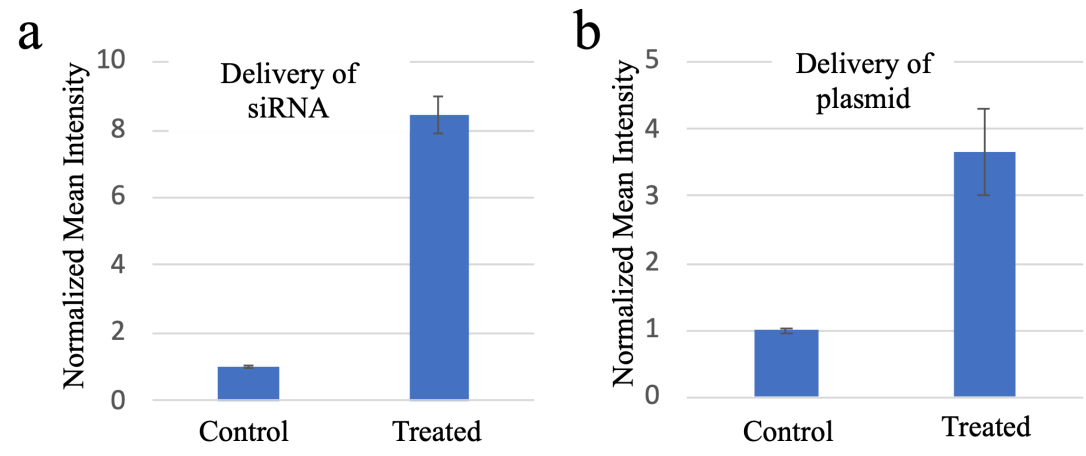

Figure E.5 Intracellular delivery of genes. Delivery results of (a) siRNA and (c) plasmid. Treatments are conducted on MDA-MB-231 cells using a 20 min continuous square-wave at $1 \mathrm{~W}$ input power. The mean intensities are normalized by the values of control experiments. The error bars indicate standard deviation of three independent experiments. 


\section{Appendix F: Journal Licenses}

\section{F.1 Journal Permission for the Article Presented in Chapter 2}

Salari, A., Gnyawali, V., Griffiths, I.M., Karshafian, R., Kolios, M.C., and Tsai, S.S.H. Shrinking microbubbles with microfluidics: Mathematical modelling to control microbubble sizes. Soft Matter 13, 8796-8806 (2017). DOI: 10.1039/C7SM01418J

Here is the permission policy for reproducing articles published in the Royal Society of Chemistry:

“Authors contributing to RSC publications (journal articles, books or book chapters) do not need to formally request permission to reproduce material contained in this article provided that the correct acknowledgement is given with the reproduced material. If you are the author of this article you still need to obtain permission to reproduce the whole article in a third party publication with the exception of reproduction of the whole article in a thesis or dissertation."

\section{F.2 Journal Permission for the Article Presented in Chapter 3}

Salari, A.*, Xu, J.*, Kolios, M.C., and Tsai, S.S.H. Expansion-mediated breakup of bubbles and droplets in microfluidics. Phys. Rev. Fluids 5, 013602 (2020). (* represents equal contribution)

Here is the license agreement for reproducing the above-mentioned article in this dissertation: 


\section{APS American Physical Society physics "iseuse and Permissions License}

04-Mar-2020

This license agreement between the American Physical Society ("APS") and Alinaghi Salari ("You") consists of your license details and the terms and conditions provided by the American Physical Society and SciPris.

Licensed Content Information

License Number:

RNP/20/MAR/023426

License date:

04-Mar-2020

DOI:

10.1103/PhysRevFluids.5.013602

Title:

Expansion-mediated breakup of bubbles and droplets in microfluidics

Author:

Alinaghi Salari et al.

Publication:

Physical Review Fluids

Publisher:

American Physical Society

Cost:

USD $\$ 0.00$

\section{$\underline{\text { Request Details }}$}

Does your reuse require significant modifications: No

Specify intended distribution Worldwide

locations:

Reuse in a thesis/dissertation

Reuse Category:

Author of requested content

Requestor Type:

Whole Article

Items for Reuse:

Electronic and Print

Format for Reuse:

More Than 10000

Total number of print copies:

Information about New Publication:

University/Publisher:

Title of dissertation/thesis:

Author(s):

Expected completion date:

License Reques tor Information

Name:

Affiliation:

Email Id:

Country:
Ryerson University

Acoustofluidics for Diagnostics and Intracellular Delivery

Alinaghi Salari

Jun. 2020

Alinaghi Salari

Individual

ai.ni.salar@gmail.com

Canada 


\section{APS American Phsical Society physics seuse and Permissions License}

\section{TERMS AND CONDITIONS}

The American Physical Society (APS) is pleased to grant the Requestor of this license a non-exclusive, non-transferable permission, limited to Electronic and Print format, provided all criteria out lined below are followed.

1. You must also obtain permission from at least one of the lead authors for each separate work, if you haven't done so already. The author's name and affiliation can be found on the first page of the published Article.

2. For electronic format permissions, Requestor agrees to provide a hyperlink from the reprinted APS material using the source material's DOI on the web page where the work appears. The hyperlink should use the standard DOI resolution URL, http://dx.doi.org/\{DOI $\}$. The hyperlink may be embedded in the copyright credit line.

3. For print format permissions, Requestor agrees to print the required copyright credit line on the first page where the material appears: "Reprinted (abstract/excerpt/figure) with permission from [(FULL REFERENCE CITAT ION) as follows: Author's Names, APS Journal Title, Vlume Number, Page Number and Year of Publication.] Copyright (YEAR) by the American Physical Society."

4. Permission granted in this license is for a one-time use and does not include permission for any future editions, updates, databases, formats or other matters. Permission must be sought for any additional use.

5. Use of the material does not and must not imply any endorsement by APS.

6. APS does not imply, purport or intend to grant permission to reuse materials to which it does not hold copyright. It is the requestor's sole responsibility to ensure the licensed material is original to APS and does not contain the copyright of another entity, and that the copyright notice of the figure, photograph, cover or table does not indicate it was reprinted by APS with permission from another source.

7. The permission granted herein is personal to the Requestor for the use specified and is not transferable or assignable without express written permission of APS. This license may not be amended except in writing by APS.

8. You may not alter, edit or modify the material in any manner.

9. You may translate the materials only when translation rights have been granted.

10. APS is not responsible for any errors or omissions due to translation.

11. You may not use the material for promotional, sales, advertising or marketing purposes.

12. The foregoing license shall not take effect unless and until APS or its agent, Aptara, receives payment in full in accordance with Aptara Billing and Payment Terms and Conditions, which are incorporated herein by reference.

13. Should the terms of this license be violated at any time, APS or Aptara may revoke the license with no refund to you and seek relief to the fullest extent of the laws of the USA. Official written notice will be made using the contact information provided with the permission request. Failure to receive such notice will not nullify revocation of the permission.

14. APS reserves all rights not specifically granted herein.

15. This document, including the Aptara Billing and Payment Terms and Conditions, shall be the entire agreement between the parties relating to the subject matter hereof.

\section{F.3 Journal Permission for the Article Presented in Chapter 5}




\section{JOHN WILEY AND SONS LICENSE}

TERMS AND CONDITIONS

Mar 14, 2020

This Agreement between Ali Salari ("You") and John Wiley and Sons ("John Wiley and Sons") consists of your license details and the terms and conditions provided by John Wiley and Sons and Copyright Clearance Center.

License Number

License date

Licensed Content

Publisher

Licensed Content

Publication

Licensed Content Title
4787721118041

Mar 14, 2020

John Wiley and Sons

Small

Dancing with the Cells: Acoustic Microflows Generated by Oscillating Cells

Licensed Content Author

Alinaghi Salari, Sila Appak-Baskoy, Maya Ezzo, et al

Licensed Content Date

Dec 12, 2019

Licensed Content Volume

16

Licensed Content Issue

9

Licensed Content Pages

7

Type of use

Dissertation/Thesis

Requestor type
Author of this Wiley article 
Format

Portion

Will you be translating?

Title of your thesis / dissertation

Expected completion date

Expected size (number of pages)

$\begin{array}{ll} & \begin{array}{l}\text { Ali Salari } \\ \text { 33 Isabella Street } \\ \text { Apt. 2315 }\end{array} \\ \text { Requestor Location } & \begin{array}{l}\text { Toronto, ON M4Y 2P7 } \\ \text { Canada } \\ \text { Attn: Ali Salari }\end{array} \\ \text { Publisher Tax ID } & \text { EU826007151 } \\ \text { Total } & 0.00 \text { CAD } \\ \text { Terms and Conditions } & \end{array}$

\section{TERMS AND CONDITIONS}

This copyrighted material is owned by or exclusively licensed to John Wiley \& Sons, Inc. or one of its group companies (each a"Wiley Company") or handled on behalf of a society with which a Wiley Company has exclusive publishing rights in relation to a particular work (collectively "WILEY"). By clicking "accept" in connection with completing this licensing transaction, you agree that the following terms and conditions apply to this transaction (along with the billing and payment terms and conditions established by the Copyright Clearance Center Inc., ("CCC's Billing and Payment terms and conditions"), at the time that you opened your RightsLink account (these are available at any time at http://myaccount.copyright.com).

\section{Terms and Conditions}


- The materials you have requested permission to reproduce or reuse (the "Wiley Materials") are protected by copyright.

- You are hereby granted a personal, non-exclusive, non-sub licensable (on a standalone basis), non-transferable, worldwide, limited license to reproduce the Wiley. Materials for the purpose specified in the licensing process. This license, and any CONTENT (PDF or image file) purchased as part of your order, is for a one-time use only and limited to any maximum distribution number specified in the license. The first instance of republication or reuse granted by this license must be completed within two years of the date of the grant of this license (although copies prepared before the end date may be distributed thereafter). The Wiley Materials shall not be used in any other manner or for any other purpose, beyond what is granted in the license. Permission is granted subject to an appropriate acknowledgement given to the author, title of the material/book/journal and the publisher. You shall also duplicate the copyright notice that appears in the Wiley publication in your use of the Wiley Material. Permission is also granted on the understanding that nowhere in the text is a previously published source acknowledged for all or part of this Wiley Material. Any third party content is expressly excluded from this permission.

- With respect to the Wiley Materials, all rights are reserved. Except as expressly granted by the terms of the license, no part of the Wiley Materials may be copied, modified, adapted (except for minor reformatting required by the new Publication), translated, reproduced, transferred or distributed, in any form or by any means, and no derivative works may be made based on the Wiley Materials without the prior permission of the respective copyright owner.For STM Signatory Publishers clearing permission under the terms of the STM Permissions Guidelines only, the terms of the license are extended to include subsequent editions and for editions in other languages, provided such editions are for the work as a whole in situ and does not involve the separate exploitation of the permitted figures or extracts, You may not alter, remove or suppress in any manner any copyright, trademark or other notices displayed by the Wiley Materials. You may not license, rent, sell, loan, lease, pledge, offer as security, transfer or assign the Wiley Materials on a stand-alone basis, or any of the rights granted to you hereunder to any other person.

- The Wiley Materials and all of the intellectual property rights therein shall at all times remain the exclusive property of John Wiley \& Sons Inc, the Wiley Companies, or their respective licensors, and your interest therein is only that of having possession of and the right to reproduce the Wiley Materials pursuant to Section 2 herein during the continuance of this Agreement. You agree that you own no right, title or interest in or to the Wiley Materials or any of the intellectual property rights therein. You shall have no rights hereunder other than the license as provided for above in Section 2. No right, license or interest to any trademark, trade name, service mark or other branding ("Marks") of WILEY or its licensors is granted hereunder, and you agree that you shall not assert any such right, license or interest with respect thereto

- NEITHER WILEY NOR ITS LICENSORS MAKES ANY WARRANTY OR REPRESENTATION OF ANY KIND TO YOU OR ANY THIRD PARTY, EXPRESS, IMPLIED OR STATUTORY, WITH RESPECT TO THE MATERIALS OR THE ACCURACY OF ANY INFORMATION CONTAINED IN THE MATERIALS, INCLUDING, WITHOUT LIMITATION, ANY IMPLIED WARRANTY OF MERCHANTABILITY, ACCURACY, SATISFACTORY QUALITY, FITNESS FOR A PARTICULAR PURPOSE, USABILITY, INTEGRATION OR NON-INFRINGEMENT AND ALL SUCH WARRANTIES ARE HEREBY EXCLUDED BY WILEY AND ITS LICENSORS AND WAIVED 
BY YOU.

- WILEY shall have the right to terminate this Agreement immediately upon breach of this Agreement by you.

- You shall indemnify, defend and hold harmless WILEY, its Licensors and their respective directors, officers, agents and employees, from and against any actual or threatened claims, demands, causes of action or proceedings arising from any breach of this Agreement by you.

- IN NO EVENT SHALL WILEY OR ITS LICENSORS BE LIABLE TO YOU OR ANY OTHER PARTY OR ANY OTHER PERSON OR ENTITY FOR ANY SPECIAL, CONSEQUENTIAL, INCIDENTAL, INDIRECT, EXEMPLARY OR PUNITIVE DAMAGES, HOWEVER CAUSED, ARISING OUT OF OR IN CONNECTION WITH THE DOWNLOADING, PROVISIONING, VIEWING OR USE OF THE MATERIALS REGARDLESS OF THE FORM OF ACTION, WHETHER FOR BREACH OF CONTRACT, BREACH OF WARRANTY, TORT, NEGLIGENCE, INFRINGEMENT OR OTHERWISE (INCLUDING, WITHOUT LIMITATION, DAMAGES BASED ON LOSS OF PROFITS, DATA, FILES, USE, BUSINESS OPPORTUNITY OR CLAIMS OF THIRD PARTIES), AND WHETHER OR NOT THE PARTY HAS BEEN ADVISED OF THE POSSIBILITY OF SUCH DAMAGES. THIS LIMITATION SHALL APPLY NOTWITHSTANDING ANY FAILURE OF ESSENTIAL PURPOSE OF ANY LIMITED REMEDY PROVIDED HEREIN.

- Should any provision of this Agreement be held by a court of competent jurisdiction to be illegal, invalid, or unenforceable, that provision shall be deemed amended to achieve as nearly as possible the same economic effect as the original provision, and the legality, validity and enforceability of the remaining provisions of this Agreement shall not be affected or impaired thereby.

- The failure of either party to enforce any term or condition of this Agreement shall not constitute a waiver of either party's right to enforce each and every term and condition of this Agreement. No breach under this agreement shall be deemed waived or excused by either party unless such waiver or consent is in writing signed by the party granting such waiver or consent. The waiver by or consent of a party to a breach of any provision of this Agreement shall not operate or be construed as a waiver of or consent to any other or subsequent breach by such other party.

- This Agreement may not be assigned (including by operation of law or otherwise) by you without WILEY's prior written consent.

- Any fee required for this permission shall be non-refundable after thirty (30) days from receipt by the CCC.

- These terms and conditions together with CCC's Billing and Payment terms and conditions (which are incorporated herein) form the entire agreement between you and WILEY concerning this licensing transaction and (in the absence of fraud) supersedes all prior agreements and representations of the parties, oral or written. This Agreement may not be amended except in writing signed by both parties. This Agreement shall be binding upon and inure to the benefit of the parties' successors, legal representatives, and authorized assigns. 
- In the event of any conflict between your obligations established by these terms and conditions and those established by CCC's Billing and Payment terms and conditions, these terms and conditions shall prevail.

- WILEY expressly reserves all rights not specifically granted in the combination of (i) the license details provided by you and accepted in the course of this licensing transaction, (ii) these terms and conditions and (iii) CCC's Billing and Payment terms and conditions.

- This Agreement will be void if the Type of Use, Format, Circulation, or Requestor Type was misrepresented during the licensing process.

- This Agreement shall be governed by and construed in accordance with the laws of the State of New York, USA, without regards to such state's conflict of law rules. Any legal action, suit or proceeding arising out of or relating to these Terms and Conditions or the breach thereof shall be instituted in a court of competent jurisdiction in New York County in the State of New York in the United States of America and each party hereby consents and submits to the personal jurisdiction of such court, waives any objection to venue in such court and consents to service of process by registered or certified mail, return receipt requested, at the last known address of such party.

\section{WILEY OPEN ACCESS TERMS AND CONDITIONS}

Wiley Publishes Open Access Articles in fully Open Access Journals and in Subscription journals offering Online Open. Although most of the fully Open Access journals publish open access articles under the terms of the Creative Commons Attribution (CC BY) License only, the subscription journals and a few of the Open Access Journals offer a choice of Creative Commons Licenses. The license type is clearly identified on the article.

\section{The Creative Commons Attribution License}

The Creative Commons Attribution License (CC-BY) allows users to copy, distribute and transmit an article, adapt the article and make commercial use of the article. The CC-BY license permits commercial and non-

\section{Creative Commons Attribution Non-Commercial License}

The Creative Commons Attribution Non-Commercial (CC-BY-NC)License permits use, distribution and reproduction in any medium, provided the original work is properly cited and is not used for commercial purposes.(see below)

\section{Creative Commons Attribution-Non-Commercial-NoDerivs License}

The Creative Commons Attribution Non-Commercial-NoDerivs License (CC-BY-NC-ND) permits use, distribution and reproduction in any medium, provided the original work is properly cited, is not used for commercial purposes and no modifications or adaptations are made. (see below)

\section{Use by commercial "for-profit" organizations}

Use of Wiley Open Access articles for commercial, promotional, or marketing purposes requires further explicit permission from Wiley and will be subject to a fee. 


\section{F.4 Journal Permissions for the Figures Reproduced in Chapter 1}

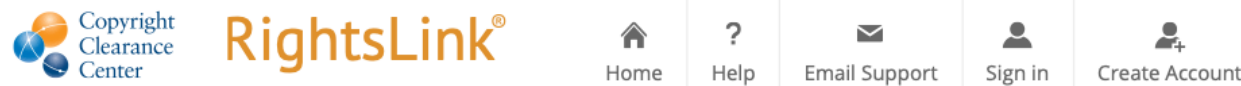

\begin{tabular}{|c|c|}
\hline & $\begin{array}{l}\text { Microbubble compositions, properties and biomedical } \\
\text { applications }\end{array}$ \\
\hline & Author: S. R. Sirsi, , M. A. Borden \\
\hline$\Rightarrow$ Taylor \& Francis & Publication: Bubble Science, Engineering and Technology \\
\hline Taylor \& Francis Group & Publisher: Taylor \& Francis \\
\hline & Date: Nov 1, 2009 \\
\hline & Rights managed by Taylor \& Francis \\
\hline \multicolumn{2}{|c|}{ Thesis/Dissertation Reuse Request } \\
\hline \multicolumn{2}{|c|}{$\begin{array}{l}\text { Taylor \& Francis is pleased to offer reuses of its content for a thesis or dissertation free of charge contingent on } \\
\text { resubmission of permission request if work is published. }\end{array}$} \\
\hline BACK & CLOSE \\
\hline
\end{tabular}

() 2020 Copyright - All Rights Reserved | Copyright Clearance Center, Inc. | Privacy statement | Terms and Conditions Comments? We would like to hear from you. E-mail us at customercare@copyright.com 
SPRINGER NATURE LICENSE

TERMS AND CONDITIONS

Mar 18, 2020

This Agreement between Ali Salari ("You") and Springer Nature ("Springer Nature") consists of your license details and the terms and conditions provided by Springer Nature and Copyright Clearance Center.

License Number

License date

Licensed Content Publisher

Licensed Content Publication

Licensed Content Title

Licensed Content Author

Licensed Content Date

Type of Use

Requestor type

Format

Portion

Number of

figures/tables/illustrations

High-res required
4791750432968

Mar 18, 2020

Springer Nature

Nature

In vitro and ex vivo strategies for intracellular delivery

Martin P. Stewart et al

Oct 12,2016

Thesis/Dissertation

academic/university or research institute

print and electronic

figures/tables/illustrations

1

no 
Will you be translating?

Circulation/distribution

Author of this Springer Nature content

Title

Institution name

Expected presentation date

Portions

Requestor Location

Total

Terms and Conditions no

50000 or greater

no

Acoustofluidics for Diagnostics and Intracellular Delivery

Ryerson University

Jun 2020

Figure 3

\section{Ali Salari}

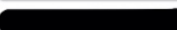

Toronto, ON M4Y 2P7

Canada

Attn: Ali Salari

0.00 USD

\section{Springer Nature Customer Service Centre GmbH Terms and Conditions}

This agreement sets out the terms and conditions of the licence (the Licence) between you and Springer Nature Customer Service Centre GmbH (the Licensor). By clicking 'accept' and completing the transaction for the material (Licensed Material), you also confirm your acceptance of these terms and conditions.

\section{Grant of License}

1. 1. The Licensor grants you a personal, non-exclusive, non-transferable, world-wide licence to reproduce the Licensed Material for the purpose specified in your order only. Licences are granted for the specific use requested in the order and for no other use, subject to the conditions below. 
1. 2. The Licensor warrants that it has, to the best of its knowledge, the rights to license reuse of the Licensed Material. However, you should ensure that the material you are requesting is original to the Licensor and does not carry the copyright of another entity (as credited in the published version).

1.3. If the credit line on any part of the material you have requested indicates that it was reprinted or adapted with permission from another source, then you should also seek permission from that source to reuse the material.

\section{Scope of Licence}

2. 1. You may only use the Licensed Content in the manner and to the extent permitted by these Ts\&Cs and any applicable laws.

2. 2. A separate licence may be required for any additional use of the Licensed Material, e.g. where a licence has been purchased for print only use, separate permission must be obtained for electronic re-use. Similarly, a licence is only valid in the language selected and does not apply for editions in other languages unless additional translation rights have been granted separately in the licence. Any content owned by third parties are expressly excluded from the licence.

2.3. Similarly, rights for additional components such as custom editions and derivatives require additional permission and may be subject to an additional fee. Please apply to Journalpermissions@springernature.com/bookpermissions@springernature.com for these rights.

2. 4. Where permission has been granted free of charge for material in print, permission may also be granted for any electronic version of that work, provided that the material is incidental to your work as a whole and that the electronic version is essentially equivalent to, or substitutes for, the print version.

2.5. An alternative scope of licence may apply to signatories of the STM Permissions Guidelines, as amended from time to time.

\section{Duration of Licence}

3. 1. A licence for is valid from the date of purchase ('Licence Date') at the end of the relevant period in the below table:

\begin{tabular}{|l|l|}
\hline Scope of Licence & Duration of Licence \\
\hline Post on a website & 12 months \\
\hline Presentations & 12 months \\
\hline Books and journals & Lifetime of the edition in the language purchased \\
\hline
\end{tabular}

\section{Acknowledgement}

4. 1. The Licensor's permission must be acknowledged next to the Licenced Material in print. In electronic form, this acknowledgement must be visible at the same time as the figures/tables/illustrations or abstract, and must be hyperlinked to the journal/book's 
homepage. Our required acknowledgement format is in the Appendix below.

\section{Restrictions on use}

5.1. Use of the Licensed Material may be permitted for incidental promotional use and minor editing privileges e.g. minor adaptations of single figures, changes of format, colour and/or style where the adaptation is credited as set out in Appendix 1 below. Any other changes including but not limited to, cropping, adapting, omitting material that affect the meaning, intention or moral rights of the author are strictly prohibited.

5. 2. You must not use any Licensed Material as part of any design or trademark.

5. 3. Licensed Material may be used in Open Access Publications (OAP) before publication by Springer Nature, but any Licensed Material must be removed from OAP sites prior to final publication.

\section{Ownership of Rights}

6. 1. Licensed Material remains the property of either Licensor or the relevant third party and any rights not explicitly granted herein are expressly reserved.

\section{Warranty}

IN NO EVENT SHALL LICENSOR BE LIABLE TO YOU OR ANY OTHER PARTY OR ANY OTHER PERSON OR FOR ANY SPECIAL, CONSEQUENTIAL, INCIDENTAL OR INDIRECT DAMAGES, HOWEVER CAUSED, ARISING OUT OF OR IN CONNECTION WITH THE DOWNLOADING, VIEWING OR USE OF THE MATERIALS REGARDLESS OF THE FORM OF ACTION, WHETHER FOR BREACH OF CONTRACT, BREACH OF WARRANTY, TORT, NEGLIGENCE, INFRINGEMENT OR OTHERWISE (INCLUDING, WITHOUT LIMITATION, DAMAGES BASED ON LOSS OF PROFITS, DATA, FILES, USE, BUSINESS OPPORTUNITY OR CLAIMS OF THIRD PARTIES), AND

WHETHER OR NOT THE PARTY HAS BEEN ADVISED OF THE POSSIBILITY OF SUCH DAMAGES. THIS LIMITATION SHALL APPLY NOTWITHSTANDING ANY FAILURE OF ESSENTIAL PURPOSE OF ANY LIMITED REMEDY PROVIDED HEREIN.

\section{Limitations}

8. 1. BOOKS ONLY:Where 'reuse in a dissertation/thesis' has been selected the following terms apply: Print rights of the final author's accepted manuscript (for clarity, NOT the published version) for up to 100 copies, electronic rights for use only on a personal website or institutional repository as defined by the Sherpa guideline (www.sherpa.ac.uk/romeo/). 


\section{Termination and Cancellation}

9. 1. Licences will expire after the period shown in Clause 3 (above).

9. 2. Licensee reserves the right to terminate the Licence in the event that payment is not received in full or if there has been a breach of this agreement by you.

\section{Appendix 1 - Acknowledgements:}

For Journal Content:

Reprinted by permission from [the Licensor]: [Journal Publisher (e.g. Nature/Springer/Palgrave)] [JOURNAL NAME] [REFERENCE CITATION (Article name, Author(s) Name), [COPYRIGHT] (year of publication)

For Advance Online Publication papers:

Reprinted by permission from [the Licensor]: [Journal Publisher (e.g. Nature/Springer/Palgrave)] [JOURNAL NAME] [REFERENCE CITATION (Article name, Author(s) Name), [COPYRIGHT] (year of publication), advance online publication, day month year (doi: 10.1038/sj.[JOURNAL ACRONYM].)

For Adaptations/Translations: Adapted/Translated by permission from [the Licensor]: [Journal Publisher (e.g. Nature/Springer/Palgrave)] [JOURNAL NAME] [REFERENCE CITATION (Article name, Author(s) Name), [COPYRIGHT] (year of publication)

Note: For any republication from the British Journal of Cancer,the following credit line style applies:

Reprinted/adapted/translated by permission from [the Licensor]: on behalf of Cancer Research UK: : [Journal Publisher (e.g. Nature/Springer/Palgrave)] [JOURNAL NAME] [REFERENCE CITATION (Article name, Author(s) Name), [COPYRIGHT] (year of publication)

For Advance Online Publication papers:

Reprinted by permission from The [the Licensor]: on behalf of Cancer Research UK: [Journal Publisher (e.g. Nature/Springer/Palgrave)] [JOURNAL NAME]

[REFERENCE CITATION (Article name, Author(s) Name), [COPYRIGHT] (year of publication), advance online publication, day month year (doi: 10.1038/sj.

[JOURNAL ACRONYM])

For Book content:

Reprinted/adapted by permission from [the Licensor]: [Book Publisher (e.g. Palgrave Macmillan, Springer etc) [Book Title] by [Book author(s)]

[COPYRIGHT] (year of publication)

Other Conditions:

Version 1.2 


\begin{tabular}{|c|c|}
\hline \multirow{6}{*}{ स्बु ACS Publications } & $\begin{array}{l}\text { Nanostraw-Electroporation System for Highly Efficient } \\
\text { Intracellular Delivery and Transfection }\end{array}$ \\
\hline & Author: Xi Xie, Alexander M. Xu, Sergio Leal-Ortiz, et al \\
\hline & Publication: ACS Nano \\
\hline & Publisher: American Chemical Society \\
\hline & Date: May 1, 2013 \\
\hline & Copyright @ 2013, American Chemical Society \\
\hline
\end{tabular}

\section{PERMISSION/LICENSE IS GRANTED FOR YOUR ORDER AT NO CHARGE}

This type of permission/license, instead of the standard Terms \& Conditions, is sent to you because no fee is being charged for your order. Please note the following:

- Permission is granted for your request in both print and electronic formats, and translations.

- If figures and/or tables were requested, they may be adapted or used in part.

- Please print this page for your records and send a copy of it to your publisher/graduate school.

- Appropriate credit for the requested material should be given as follows: "Reprinted (adapted) with permission from (COMPLETE REFERENCE CITATION). Copyright (YEAR) American Chemical Society." Insert appropriate

information in place of the capitalized words.

- One-time permission is granted only for the use specified in your request. No additional uses are granted (such as derivative works or other editions). For any other uses, please submit a new request.

If credit is given to another source for the material you requested, permission must be obtained from that source.

BACK

CLOSE WINDOW

(c) 2020 Copyright - All Rights Reserved | Copyright Clearance Center, Inc. | Privacy statement | Terms and Conditions Comments? We would like to hear from you. E-mail us at customercare@copyright.com 


\section{Marketplace $^{\mathrm{Tm}}$}

Order Number: 1023603

Order Date: 18 Mar 2020

\section{Payment Information}

Ali Salari

ai.ni.salar@gmail.com

Payment method: Invoice

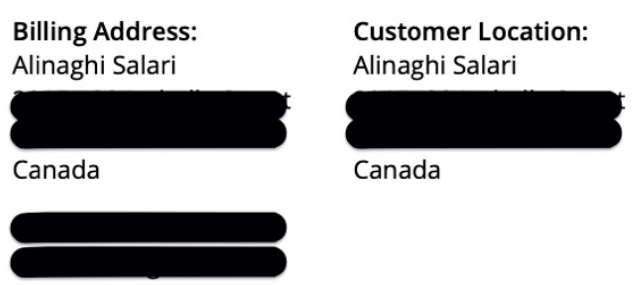

\section{Order Details}

\section{Lab on a chip}

Billing Status: Open

Order license ID

1023603-1

Order detail status

Completed

ISSN

1473-0189

Type of use

Republish in a thesis/dissertation

Publisher

ROYAL SOCIETY OF CHEMISTRY

Portion

Image/photo/illustration

0.00 USD

\section{LICENSED CONTENT}

Publication Title

Author/Editor

Date

Language
Lab on a chip

Royal Society of Chemistry (Great Britain)

01/01/2001

English
Country

Publication Type

URL
United Kingdom of Great Britain and Northern Ireland

Royal Society of Chemistry

e-Journal

http://www.rsc.org/loc

\section{REQUEST DETAILS}

Portion Type

Number of images I photos / illustrations

Format (select all that apply)
Image/photo/illustratio

n

1

Print,Electronic

\section{Distribution \\ Translation}

Copies for the disabled?

Minor editing privileges?

\section{Worldwide}

Original language of publication

No

Yes 


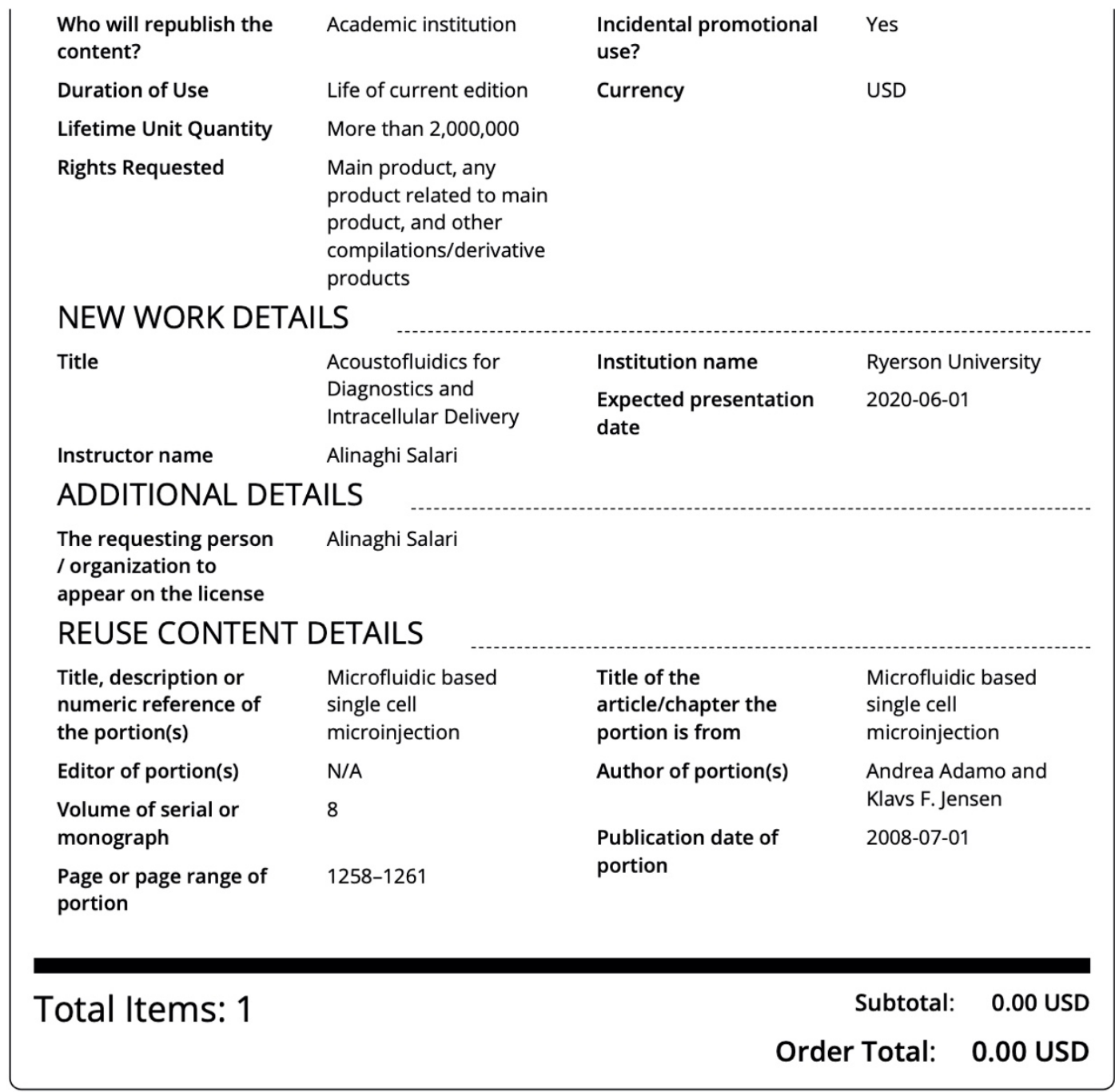




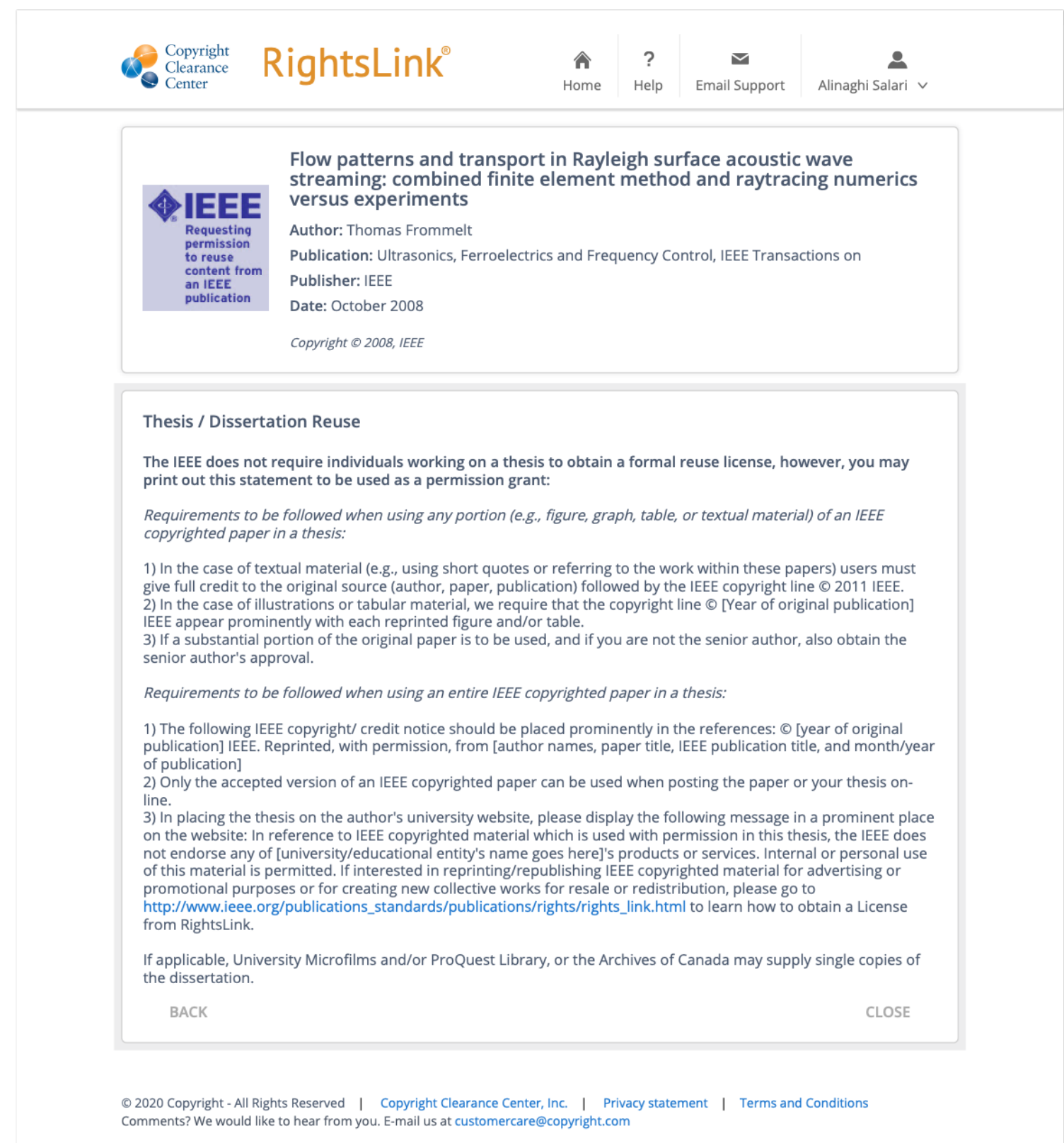

Comments? We would like to hear from you. E-mail us at customercare@copyright.com 


\section{References}

1. Lazzari, L. et al. Ultrasound elastography techniques for diagnosis and follow-up of hepatic venoocclusive disease. Bone Marrow Transplant. 54, 1145-1147 (2019).

2. Lindner, J. R. Microbubbles in medical imaging: Current applications and future directions. Nat. Rev. Drug Discov. 3, 527-533 (2004).

3. Ibsen, S., Schutt, C. E. \& Esener, S. Microbubble-mediated ultrasound therapy: A review of its potential in cancer treatment. Drug Des. Devel. Ther. 7, 375-388 (2013).

4. Fechheimer, M. et al. Transfection of mammalian cells with plasmid DNA by scrape loading and sonication loading. Proc. Natl. Acad. Sci. U. S. A. 84, 8463-8467 (1987).

5. Prentice, P., Cuschieri, A., Dholakia, K., Prausnitz, M. \& Campbell, P. Membrane disruption by optically controlled microbubble cavitation. Nat. Phys. 1, 107-110 (2005).

6. Qin, P. et al. The relationship between microbubble size and heterogeneous sonoporation at the single-cell level. in IEEE International Ultrasonics Symposium, IUS 2-5 (2016). doi:10.1109/ULTSYM.2016.7728424

7. Yuan, F., Yang, C. \& Zhong, P. Cell membrane deformation and bioeffects produced by tandem bubble-induced jetting flow. Proc. Natl. Acad. Sci. U. S. A. 112, E7039-47 (2015).

8. Li, J., Xi, A., Qiao, H. \& Liu, Z. Ultrasound-mediated diagnostic imaging and advanced treatment with multifunctional micro/nanobubbles. Cancer Lett. 475, 92-98 (2020).

9. Sirsi, S. \& Borden, M. Microbubble compositions, properties and biomedical applications. Bubble Sci Eng Technol. 1, 3-17 (2010).

10. Sirsi, S., Feshitan, J., Kwan, J., Homma, S. \& Borden, M. Effect of microbubble size on fundamental mode high frequency ultrasound imaging in mice. Ultrasound Med. Biol. 36, 935-948 (2010).

11. Daeichin, V. et al. Microbubble composition and preparation for high-frequency contrast-enhanced ultrasound imaging: in vitro and in vivo evaluation. IEEE Trans. Ultrason. Ferroelectr. Freq. Control 64, 1-1 (2016).

12. Hettiarachchi, K., Talu, E., Longo, M. L., Dayton, P. A. \& Lee, A. P. On-chip generation of 
microbubbles as a practical technology for manufacturing contrast agents for ultrasonic imaging. Lab Chip 7, 463-8 (2007).

13. Garstecki, P. et al. Formation of monodisperse bubbles in a microfluidic flow-focusing device. Appl. Phys. Lett. 85, 2649-2651 (2004).

14. Garstecki, P., Gañán-Calvo, a M. \& Whitesides, G. M. Formation of bubbles and droplets in microfluidic systems. Bull. Polish Acad. Sci. 53, 361-372 (2005).

15. Liu, G., Wu, Z. \& Craig, V. S. J. Cleaning of protein-coated surfaces using nanobubbles: An investigation using a Quartz Crystal Microbalance. J. Phys. Chem. C 112, 16748-16753 (2008).

16. de Leon, A. et al. Ultrasound contrast agents and delivery systems in cancer detection and therapy. Advances in Cancer Research 139, (Elsevier Inc., 2018).

17. Wang, Q. et al. Generation and stability of size-adjustable bulk nanobubbles based on periodic pressure change. Sci. Rep. 9, 1-9 (2019).

18. Stewart, M. P. et al. In vitro and ex vivo strategies for intracellular delivery. Nature 538, 183-192 (2016).

19. Stewart, M. P., Langer, R. \& Jensen, K. F. Intracellular delivery by membrane disruption: Mechanisms, strategies, and concepts. Chem. Rev. 118, 7409-7531 (2018).

20. Chow, Y. T. et al. Single cell transfection through precise microinjection with quantitatively controlled injection volumes. Sci. Rep. 6, 1-9 (2016).

21. Zhang, Y. \& Yu, L. C. Single-cell microinjection technology in cell biology. BioEssays 30, 606610 (2008).

22. Covello, G., Siva, K., Adami, V. \& Denti, M. A. An electroporation protocol for efficient DNA transfection in PC12 cells. Cytotechnology 66, 543-553 (2014).

23. Li, F. et al. Efficient transfection of DNA into primarily cultured rat sertoli cells by electroporation. Biol Reprod. 88, 61 (2013).

24. Jordan, E. T., Collins, M., Terefe, J., Ugozzoli, L. \& Rubio, T. Optimizing electroporation conditions in primary and other difficult-to-transfect cells. J. Biomol. Tech. 19, 328-334 (2008).

25. Mitchell, M. J., Castellanos, C. A. \& King, M. R. Nanostructured surfaces to target and kill circulating tumor cells while repelling leukocytes. J. Nanomater. 2012, (2012).

26. He, X., Amin, A. A., Fowler, A. \& Toner, M. Thermally induced introduction of trehalose into primary rat hepatocytes. Cell Preserv. Technol. 4, 178-187 (2006).

27. Schomaker, M. et al. Characterization of nanoparticle mediated laser transfection by femtosecond laser pulses for applications in molecular medicine. J. Nanobiotechnology 13, 10 (2015).

28. Vogel, A., Noack, J., Hüttman, G. \& Paltauf, G. Mechanisms of femtosecond laser nanosurgery of cells and tissues. Appl. Phys. B 81, 1015-1047 (2005). 
29. Barber, M. A. A Technic for the inoculation of bacteria and other substances into living cells. $J$. Infect. Dis. 8, 348-360 (1911).

30. Yin, H. et al. Non-viral vectors for gene-based therapy. Nat. Rev. Genet. 15, 541-555 (2014).

31. Stewart, M. P., Lorenz, A., Dahlman, J. \& Sahay, G. Challenges in carrier-mediated intracellular delivery: Moving beyond endosomal barriers. Wiley Interdiscip. Rev. Nanomedicine Nanobiotechnology 8, 465-478 (2016).

32. Hapala, I. Breaking the barrier: Methods for reversible permeabilization of cellular membranes. Crit. Rev. Biotechnol. 17, 105-122 (1997).

33. Permeability barriers formed by membrane lipids. Bioelectrochemistry Bioenerg. 27, 1-10 (1992).

34. Kido, H. et al. A novel, compact disk-like centrifugal microfluidics system for cell lysis and sample homogenization. Colloids Surfaces B Biointerfaces 58, 44-51 (2007).

35. Clarke, M. S. \& McNeil, P. L. Syringe loading introduces macromolecules into living mammalian cell cytosol. J. Cell Sci. 102, 533-541 (1992).

36. McNeil, P. L. \& Warder, E. Glass beads load macromolecules into living cells. J. Cell Sci. 88, 669678 (1987).

37. McKnight, T. E. et al. Intracellular integration of synthetic nanostructures with viable cells for controlled biochemical manipulation. Nanotechnology 14, 551-556 (2003).

38. Shalek, A. K. et al. Vertical silicon nanowires as a universal platform for delivering biomolecules into living cells. Proc. Natl. Acad. Sci. U. S. A. 107, 1870-1875 (2010).

39. Xie, X. et al. Nanostraw-electroporation system for highly efficient intracellular delivery and transfection. ACS Nano 7, 4351-4358 (2013).

40. Adamo, A. \& Jensen, K. F. Microfluidic based single cell microinjection. Lab Chip 8, 1258 (2008).

41. Al-Rubeai, M., Singh, M. H., Goldman, M. H. \& Emery, A. N. Death mechanisms in animal cells in conditions of intensive agitation. Biotech Bioeng 45, 463-472 (1995).

42. Sharei, A. et al. Ex vivo cytosolic delivery of functional macromolecules to immune cells. PLoS One 10, 1-12 (2015).

43. Sharei, A. et al. A vector-free microfluidic platform for intracellular delivery. Proc. Natl. Acad. Sci. U. S. A. 110, 2082-2087 (2013).

44. Szeto, G. L. et al. Microfluidic squeezing for intracellular antigen loading in polyclonal B-cells as cellular vaccines. Sci. Rep. 5, 1-13 (2015).

45. Hallow, D. M. et al. Shear-induced intracellular loading of cells with molecules by controlled microfluidics. Biotechnol. Bioeng. 99, 846-854 (2008).

46. Dreyer, L. et al. An advanced cone-and-plate reactor for the in vitro-application of shear stress on adherent cells. Clin. Hemorheol. Microcirc. 49, 391-397 (2011). 
47. Mardikar, S. H. \& Niranjan, K. Observations on the shear damage to different animal cells in a concentric cylinder viscometer. Biotechnol. Bioeng. 68, 697-704 (2000).

48. Bose, N., Zhang, X., Maiti, T. K. \& Chakraborty, S. The role of acoustofluidics in targeted drug delivery. Biomicro 9, 052609 (2015).

49. Fan, Z., Kumon, R. E. \& Deng, C. X. Mechanisms of microbubble-facilitated sonoporation for drug and gene delivery. Ther. Deliv. 5, 467-486 (2014).

50. Guzmán, H. R., Nguyen, D. X., Khan, S. \& Prausnitz, M. R. Ultrasound-mediated disruption of cell membranes. II. Heterogeneous effects on cells. J. Acoust. Soc. Am. 110, 597 (2001).

51. Kooiman, K., Vos, H. J., Versluis, M. \& De Jong, N. Acoustic behavior of microbubbles and implications for drug delivery. Adv. Drug Deliv. Rev. 72, 28-48 (2014).

52. Khanna, S., Amso, N. N., Paynter, S. J. \& Coakley, W. T. Contrast agent bubble and erythrocyte behavior in a 1.5-MHz standing ultrasound wave. Ultrasound Med. Biol. 29, 1463-70 (2003).

53. Gruenberg, J. \& Maxfield, F. R. Membrane transport in the endocytic pathway. Curr. Opin. Cell Biol. 7, 552-563 (1995).

54. Cecchelli, R., Cacan, R. \& Verbert, A. Accumulation of UDP-GlcNAc into intracellular vesicles and occurrence of a carrier-mediated transport. Study with plasma-membrane-permeabilized mouse thymocytes. Eur. J. Biochem. 153, 111-116 (1985).

55. Wan, G.-Y. et al. Recent advances of sonodynamic therapy in cancer treatment. Cancer Biol. Med. 13, 325-338 (2016).

56. Mitragotri, S., Blankschtein, D. \& Langer, R. Ultrasound-mediated transdermal protein delivery. Science (80-. ). 269, 850-853 (1995).

57. Kotopoulis, S., Dimcevski, G., Gjertsen, B. T. \& Hoem, D. Ultrasound-mediated therapy. (2014). Available at: http://www.uib.no/en/rg/ultrasound/65974/ultrasound-mediated-therapy. (Accessed: 21st December 2017)

58. Heo, J., Sachs, F., Wang, J. \& Hua, S. Z. Shear-induced volume decrease in MDCK cells. Cell Physiol Biochem 30, 395-406 (2012).

59. Chatzizisis, Y. S. et al. Role of endothelial shear stress in the natural history of coronary atherosclerosis and vascular remodeling. Molecular, cellular, and vascular behavior. J. Am. Coll. Cardiol. 49, 2379-2393 (2007).

60. White, C. R. \& Frangos, J. A. The shear stress of it all: The cell membrane and mechanochemical transduction. Philos. Trans. R. Soc. B Biol. Sci. 362, 1459-1467 (2007).

61. Shen, F. et al. Quantitation of doxorubicin uptake, efflux, and modulation of multidrug resistance (MDR) in MDR human cancer cells. J. Pharmacol. Exp. Ther. 324, 95-102 (2008).

62. Pisco, A. O., Jackson, D. A. \& Huang, S. Reduced intracellular drug accumulation in drug-resistant 
leukemia cells is not only solely due to MDR-mediated efflux but also to decreased uptake. Front. Oncol. 4, (2014).

63. Barbee, K. A., Davies, P. F. \& Lal, R. Shear stress-induced reorganization of the surface topography of living endothelial cells imaged by atomic force microscopy. Circ. Res. 74, 163-171 (1994).

64. Goldblum, S., Bae, Y. K., Hink, W. F. \& Chalmers, J. Protective effect of methylcellulose and other polymers on insect cells subjected to laminar shear stress. Biotechnol. Prog. 6, 383-390 (1990).

65. Michaels, J. D. \& Papoutsakis, E. T. Polyvinyl alcohol and polyethylene glycol as protectants against fluid-mechanical injury of freely-suspended animal cells (CRL 8018). J. Biotechnol. 19, 241-57 (1991).

66. Tharmalingam, T., Ghebeh, H., Wuerz, T. \& Butler, M. Pluronic enhances the robustness and reduces the cell attachment of mammalian cells. Mol Biotechnol 39, 167-177 (2008).

67. Chattopadhyay, D., Rathman, J. F. \& Chalmers, J. J. The protective effect of specific medium additives with respect to bubble rupture. Biotechnol. Bioeng. 45, 473-480 (1995).

68. Humphrey, V. F. Ultrasound and matter- Physical interactions. Prog. Biophys. Mol. Biol. 93, 195211 (2007).

69. Nguyen, D. X., Mcnamara, A. J. \& Prausnitz, M. R. Equilibrium loading of cells with macromolecules by ultrasound: Effects of molecular size and acoustic energy. J. Pharm. Sci. 91, 1693-1701 (2002).

70. Nama, N. et al. Numerical study of acoustophoretic motion of particles in a PDMS microchannel driven by surface acoustic waves. Lab Chip 15, 2700-2709 (2015).

71. Worden, K. Rayleigh and Lamb waves - Basic principles. Strain 37, 167-172 (2001).

72. Lamb, H. On waves in an elastic plate. Proc. R. Soc. London A Math. Phys. Eng. Sci. 93, 114-128 (1917).

73. Fu, Y. Q. Q. et al. Advances in piezoelectric thin films for acoustic biosensors, acoustofluidics and lab-on-chip applications. Prog. Mater. Sci. 89, 31-91 (2017).

74. Vanneste, J. \& Buhler, O. Streaming by leaky surface acoustic waves. Proc. R. Soc. A Math. Phys. Eng. Sci. 467, 1779-1800 (2011).

75. Frommelt, T. et al. Flow patterns and transport in Rayleigh surface acoustic wave streaming: Combined finite element method and raytracing numerics versus experiments. IEEE Trans. Ultrason. Ferroelectr. Freq. Control 55, 2298-2305 (2008).

76. Wu, J. Shear stress in cells generated by ultrasound. Prog. Biophys. Mol. Biol. 93, 363-373 (2007).

77. Wu, J., Ross, J. P. \& Chiu, J. Reparable sonoporation generated by microstreaming. J. Acoust. Soc. Am. 111, 1460-1464 (2002).

78. Ding, X. et al. Cell separation using tilted-angle standing surface acoustic waves. Proc. Natl. Acad. 
Sci. U. S. A. 111, 12992-12997 (2014).

79. Nama, N., Huang, P. H., Huang, T. J. \& Costanzo, F. Investigation of micromixing by acoustically oscillated sharp-edges. Biomicrofluidics 10, 1-17 (2016).

80. Wu, J. \& Nyborg, W. L. Ultrasound, cavitation bubbles and their interaction with cells. Adv. Drug Deliv. Rev. 60, 1103-1116 (2008).

81. Helfield, B., Chen, X., Watkins, S. C. \& Villanueva, F. S. Biophysical insight into mechanisms of sonoporation. Proc. Natl. Acad. Sci. U. S. A. 113, 9983-9988 (2016).

82. Marmottant, P. \& Hilgenfeldt, S. Controlled vesicle deformation and lysis by single oscillating bubbles. Nature 423, 153-156 (2003).

83. Longuet-Higgins, M. S. Viscous streaming from an oscillating spherical bubble. Proc. R. Soc. London A Math. Phys. Eng. Sci. 454, (1998).

84. Bruus, H. Acoustofluidics 2: Perturbation theory and ultrasound resonance modes. Lab Chip 12, 2028 (2012).

85. Guo, F. et al. Three-dimensional manipulation of single cells using surface acoustic waves. Proc. Natl. Acad. Sci. U. S. A. 113, 1522-1527 (2016).

86. Ozcelik, A. et al. Acoustofluidic rotational manipulation of cells and organisms using oscillating solid structures. Small 12, 5230 (2016).

87. Miller, D. L. Particle gathering and microstreaming near ultrasonically activated gas-filled micropores. J. Acoust. Soc. Am. 84, 1378-87 (1988).

88. Ahmed, D. et al. Rotational manipulation of single cells and organisms using acoustic waves. Nat. Commun. 7, 11085 (2016).

89. Ayan, B. et al. Acoustofluidic coating of particles and cells. Lab Chip 16, 4366-4372 (2016).

90. Gnyawali, V. et al. Honey, I shrunk the bubbles: Microfluidic vacuum shrinkage of lipid-stabilized microbubbles. Soft Matter 13, 4011-4016 (2017).

91. Xing, X., Pan, Y. \& Yobas, L. A low-backpressure single-cell point constriction for cytosolic delivery based on rapid membrane deformations. Anal Chem 90, 1836-1844 (2018).

92. Lee, J. et al. Nonendocytic delivery of functional engineered nanoparticles into the cytoplasm of live cells using a novel, high-throughput microfluidic device. Nano Lett. 12, 6322-6327 (2012).

93. Li, J. et al. Microfluidic-enabled intracellular delivery of membrane impermeable inhibitors to study target engagement in human primary cells. ACS Chem. Biol. 12, 2970-2974 (2017).

94. Salari, A. et al. Shrinking microbubbles with microfluidics: Mathematical modelling to control microbubble sizes. Soft Matter 13, 8796-8806 (2017).

95. Salari, A., Shafii, M. B. M. B. \& Shirani, S. An experimental review on microbubble generation to be used in echo-particle image vlocimetry method to determine the pipe flow velocity. J Fluid Eng 
T ASME 135, 34501 (2013).

96. Oh, J. S. et al. Drug perfusion enhancement in tissue model by steady streaming induced by oscillating microbubbles. Comput. Biol. Med. 44, 37-43 (2014).

97. Liu, Y. et al. Magnetic nanoliposomes as in situ microbubble bombers for multimodality imageguided cancer theranostics. ACS Nano 11, 1509-1519 (2017).

98. Eisenbrey, J. R. et al. Development of an ultrasound sensitive oxygen carrier for oxygen delivery to hypoxic tissue. Int. J. Pharm. 478, 361-367 (2015).

99. Dindyal, S. \& Kyriakides, C. Ultrasound microbubble contrast and current clinical applications. Recent Pat. Cardiovasc. Drug Discov. 6, 27-41 (2011).

100. Zhang, F. et al. In vitro and preliminary in vivo validation of echo particle image velocimetry in carotid vascular imaging. Ultrasound Med. Biol. 37, 450-464 (2011).

101. Siciliano, M. et al. Cardiac resynchronization therapy by multipoint pacing improves response of left ventricular mechanics and fluid dynamics: A three-dimensional and particle image velocimetry echo study. Europace 350, 2140-50 (2016).

102. Lv, Y. et al. Novel multifunctional pH-sensitive nanoparticles loaded into microbubbles as drug delivery vehicles for enhanced tumor targeting. Sci. Rep. 6, 29321 (2016).

103. Zhu, X. et al. Ultrasound triggered image-guided drug delivery to inhibit vascular reconstruction via paclitaxel-loaded microbubbles. Sci. Rep. 6, 21683 (2016).

104. Burgess, A. \& Hynynen, K. Microbubble-assisted ultrasound for drug delivery in the brain and central nervous system. in Advances in experimental medicine and biology 880, 293-308 (2016).

105. Carugo, D. et al. Modulation of the molecular arrangement in artificial and biological membranes by phospholipid-shelled microbubbles. Biomaterials 113, 105-117 (2017).

106. Sirsi, S. R. \& Borden, M. A. Advances in ultrasound mediated gene therapy using microbubble contrast agents. Theranostics 2, 1208-1222 (2012).

107. Sirsi, S. R. \& Borden, M. A. State-of-the-art materials for ultrasound-triggered drug delivery. $A d v$. Drug Deliv. Rev. 72, 3-14 (2014).

108. Wang, S. et al. Microbubble type and distribution dependence of focused ultrasound-induced bloodbrain barrier opening. Ultrasound Med. Biol. 40, 130-7 (2014).

109. Lee, M., Lee, E. Y., Lee, D. \& Park, B. J. Stabilization and fabrication of microbubbles: Applications for medical purposes and functional materials. Soft Matter 11, 2067-2079 (2015).

110. Wan, J., Bick, A., Sullivan, M. \& Stone, H. A. Controllable microfluidic production of microbubbles in water-in-oil emulsions and the formation of porous microparticles. Adv. Mater. 20, 3314-3318 (2008).

111. Parhizkar, M., Stride, E. \& Edirisinghe, M. Preparation of monodisperse microbubbles using an 
integrated embedded capillary T-junction with electrohydrodynamic focusing. Lab Chip 14, 24372446 (2014).

112. Xu, J. H., Li, S. W., Chen, G. G. \& Luo, G. S. Formation of monodisperse microbubbles in a microfluidic device. AIChE J. 52, 2254-2259 (2006).

113. Fiabane, J., Prentice, P. \& Pancholi, K. High yielding microbubble production method. Biomed Res. Int. 2016, (2016).

114. Chong, Z. Z. et al. Acoustofluidic control of bubble size in microfluidic flow-focusing configuration. Lab Chip 15, 996-999 (2015).

115. Zhang, J. M. et al. A co-flow-focusing monodisperse microbubble generator. J. Micromechanics Microengineering 24, 035008 (2014).

116. Pimentel-Domínguez, R., Hernández-Cordero, J. \& Zenit, R. Microbubble generation using fiber optic tips coated with nanoparticles. Opt. Express 20, 8732 (2012).

117. Lee, H. et al. Microbubbles used for contrast enhanced ultrasound and theragnosis: A review of principles to applications. Biomed. Eng. Lett. 7, 59-69 (2017).

118. Wu, J. \& Li, R.-K. Ultrasound-targeted microbubble destruction in gene therapy: A new tool to cure human diseases. Genes Dis. 4, 64-74 (2017).

119. Seo, M., Williams, R. \& Matsuura, N. Size reduction of cosolvent-infused microbubbles to form acoustically responsive monodisperse perfluorocarbon nanodroplets. Lab Chip 15, 3581-3590 (2015).

120. Arashiro, E. Y. \& Demarquette, N. R. Use of the pendant drop method to measure interfacial tension between molten polymers. Mater. Res. 2, 23-32 (1999).

121. Nie, Z. et al. Emulsification in a microfluidic flow-focusing device: Effect of the viscosities of the liquids. Microfluid. Nanofluidics 5, 585-594 (2008).

122. Lin, H., Chen, J. \& Chen, C. A novel technology: Microfluidic devices for microbubble ultrasound contrast agent generation. Med. Biol. Eng. Comput. 54, 1317-1330 (2016).

123. Klibanov, A. L. et al. Targeting and ultrasound imaging of microbubble-based contrast agents. Magn. Reson. Mater. Physics, Biol. Med. 8, 177-184 (1999).

124. Parhizkar, M., Edirisinghe, M. \& Stride, E. The effect of surfactant type and concentration on the size and stability of microbubbles produced in a capillary embedded T-junction device. RSC Adv. $\mathbf{5}$, 10751-10762 (2015).

125. Katiyar, A., Sarkar, K. \& Jain, P. Effects of encapsulation elasticity on the stability of an encapsulated microbubble. J. Colloid Interface Sci. 336, 519-525 (2009).

126. Kumar, K. N. \& Sarkar, K. Effects of ambient hydrostatic pressure on the material properties of the encapsulation of an ultrasound contrast microbubble. J. Acoust. Soc. Am. 138, 624-634 (2015). 
127. Ando, Y. et al. Microbubbles with a self-assembled poloxamer shell and a fluorocarbon inner gas. Langmuir 32, 12461-12467 (2016).

128. Segers, T., De Rond, L., De Jong, N., Borden, M. \& Versluis, M. Stability of monodisperse phospholipid-coated microbubbles formed by flow-focusing at high production rates. Langmuir 32, 3937-3944 (2016).

129. Kwan, J. J. \& Borden, M. A. Lipid monolayer collapse and microbubble stability. Adv. Colloid Interface Sci. 183-184, 82-99 (2012).

130. Doinikov, A. A. \& Bouakaz, A. Review of shell models for contrast agent microbubbles. IEEE Trans. Ultrason. Ferroelectr. Freq. Control 58, 981-993 (2011).

131. Dalvi, S. V. \& Joshi, J. R. Modeling of microbubble dissolution in aqueous medium. J. Colloid Interface Sci. 437, 259-269 (2015).

132. Epstein, P. S. \& Plesset, M. S. On the stability of gas bubbles in liquid-gas solutions. Appl. Sci. Res. 38, 133-141 (1950).

133. Sarkar, K., Katiyar, A. \& Jain, P. Growth and dissolution of an encapsulated contrast microbubble: Effects of encapsulation permeability. Ultrasound Med. Biol. 35, 1385-1396 (2009).

134. Shim, S., Wan, J., Hilgenfeldt, S., Panchal, P. D. \& Stone, H. A. Dissolution without disappearing: Multicomponent gas exchange for $\mathrm{CO} 2$ bubbles in a microfluidic channel. Lab Chip 14, 2428 (2014).

135. Volk, A., Rossi, M., Kähler, C. J., Hilgenfeldt, S. \& Marin, A. Growth control of sessile microbubbles in PDMS devices. Lab Chip 15, 4607-4613 (2015).

136. Charati, S. G. \& Stern, S. a. Diffusion of gases in silicone polymers: Molecular dynamics simulations. Macromolecules 31, 5529-5535 (1998).

137. Thomas, P., Raghavan, S. \& Forry, S. Regulating oxygen levels in a microfluidic device. Anal. Chem. 83, 8821-8824 (2011).

138. Xia, L., Porter, T. M. \& Sarkar, K. Interpreting attenuation at different excitation amplitudes to estimate strain-dependent interfacial rheological properties of lipid-coated monodisperse microbubbles. J. Acoust. Soc. Am. 138, 3994-4003 (2015).

139. Krasovitski, B. \& Kimmel, E. Stability of an encapsulated bubble shell. Ultrasonics 44, 216-220 (2006).

140. Shih, R., Hall, T., Hill, C. \& Lee, A. P. Scaled-up production of monodisperse, dual layer microbubbles using multi-array microfluidic module for medical imaging and drug delivery. Bubble Sci Eng Technol. 4, 12-20 (2012).

141. Segers, T. \& Versluis, M. Acoustic bubble sorting for ultrasound contrast agent enrichment. Lab Chip 14, 1705-14 (2014). 
142. Kok, M. P. et al. Bubble sorting in pinched microchannels for ultrasound contrast agent enrichment. Lab Chip 15, 3716-3722 (2015).

143. Salari, A., Xu, J., Kolios, M. C. \& Tsai, S. S. . Expansion-mediated breakup of bubbles and droplets in microfluidics. Phys. Rev. Fluids 5, 013602 (2020).

144. Rayleigh, Lord. On the instability of jets. Proc. London Math. Soc. s1-10, 4-13 (1878).

145. Garstecki, P., Fuerstman, M. J., Stone, H. A. \& Whitesides, G. M. Formation of droplets and bubbles in a microfluidic T-junction - Scaling and mechanism of break-up. Lab Chip 6, 437-446 (2006).

146. Dollet, B., Van Hoeve, W., Raven, J. P., Marmottant, P. \& Versluis, M. Role of the channel geometry on the bubble pinch-off in flow-focusing devices. Phys. Rev. Lett. 100, 1-4 (2008).

147. Leshansky, A. M. \& Pismen, L. M. Breakup of drops in a microfluidic T-junction. Phys. Fluids 21, 023303 (2009).

148. Samie, M., Salari, A. \& Shafii, M. B. M. B. Breakup of microdroplets in asymmetric T junctions. Phys Rev E 87, 053003 (2013).

149. $\mathrm{Xu}$, J. et al. Controllable microfluidic production of drug-loaded PLGA nanoparticles using partially water-miscible mixed solvent microdroplets as a precursor. Sci. Rep. 7, (2017).

150. Chen, Z. et al. Centrifugal micro-channel array droplet generation for highly parallel digital PCR. Lab Chip 17, 235-240 (2017).

151. Ferrara, K., Pollard, R. \& Borden, M. Ultrasound microbubble contrast agents: Fundamentals and application to gene and drug delivery. Annu. Rev. Biomed. Eng 9, 415-47 (2007).

152. Soli, K. W. et al. Decontamination of fresh produce by the use of slightly acidic hypochlorous water following pretreatment with sucrose fatty acid ester under microbubble generation. Food Control 21, 1240-1244 (2010).

153. Leshansky, A. M., Afkhami, S., Jullien, M. C. \& Tabeling, P. Obstructed breakup of slender drops in a microfluidic T junction. Phys. Rev. Lett. 108, 1-5 (2012).

154. Jullien, M. C., Tsang Mui Ching, M. J., Cohen, C., Menetrier, L. \& Tabeling, P. Droplet breakup in microfluidic T-junctions at small capillary numbers. Phys. Fluids 21, 072001 (2009).

155. Lespiat, R., Cohen-Addad, S. \& Höhler, R. Jamming and flow of random-close-packed spherical bubbles: An analogy with granular materials. Phys. Rev. Lett. 106, 1-4 (2011).

156. Hashimoto, M., Garstecki, P., Stone, H. A. \& Whitesides, G. M. Interfacial instabilities in a microfluidic Hele-Shaw cell. Soft Matter 4, 1403-1413 (2008).

157. Wu, Y. et al. Asymmetrical breakup of bubbles at a microfluidic T-junction divergence: Feedback effect of bubble collision. Microfluid. Nanofluidics 13, 723-733 (2012).

158. Nishimura, A., Schmit, A., Salkin, L., Courbin, L. \& Panizza, P. Breakup of confined drops against a micro-obstacle: An analytical model for the drop size distribution. Microfluid. Nanofluidics 21, 1- 
8 (2017).

159. Lu, Y., Fu, T., Zhu, C., Ma, Y. \& Li, H. Z. Dynamics of bubble breakup at a T junction. Phys. Rev. E 93, 1-12 (2016).

160. Vecchiolla, D., Giri, V. \& Biswal, S. L. Bubble-bubble pinch-off in symmetric and asymmetric microfluidic expansion channels for ordered foam generation. Soft Matter 14, 9312--9325 (2018).

161. Hashimoto, M., Garstecki, P. \& Whitesides, G. M. Synthesis of composite emulsions and complex foams with the use of microfluidic flow-focusing devices. Small 3, 1792-1802 (2007).

162. Hashimoto, M. \& Whitesides, G. M. Formation of bubbles in a multisection flow-focusing junction. Small 6, 1051-1059 (2010).

163. Hashimoto, M. et al. Formation of bubbles and droplets in parallel, coupled flow-focusing geometries. Small 4, 1795-1805 (2008).

164. Ricouvier, J., Tabeling, P. \& Yazhgur, P. Foam as a self-assembling amorphous photonic band gap material. Proc. Natl. Acad. Sci. U. S. A. 116, 9202-9207 (2019).

165. Ricouvier, J., Pierrat, R., Carminati, R., Tabeling, P. \& Yazhgur, P. Optimizing hyperuniformity in self-assembled bidisperse emulsions. Phys. Rev. Lett. 119, 1-6 (2017).

166. Xia, Y. \& Whitesides, G. M. Soft lithography. Annu. Rev. Mat. Sci. 28, 153-184 (1998).

167. Gañán-Calvo, A. M. Perfectly monodisperse microbubbling by capillary flow focusing: An alternate physical description and universal scaling. Phys. Rev. E - Stat. Nonlinear, Soft Matter Phys. 69, 13 (2004).

168. Garstecki, P., Fuerstman, M. J. \& Whitesides, G. M. Nonlinear dynamics of a flow-focusing bubble generator: An inverted dripping faucet. Phys. Rev. Lett. 94, 38-41 (2005).

169. Baroud, C. N., Gallaire, F. \& Dangla, R. Dynamics of microfluidic droplets. Lab Chip 10, 20322045 (2010).

170. Sullivan, M. T. \& Stone, H. A. The role of feedback in microfluidic flow-focusing devices. Philos. Trans. R. Soc. A Math. Phys. Eng. Sci. 366, 2131-2143 (2008).

171. Bedram, A. \& Moosavi, A. Droplet breakup in an asymmetric microfluidic T junction. Eur. Phys. J. E 34, (2011).

172. De menech, M., Garstecki, P., Jousse, F. \& Stone, H. A. Transition from squeezing to dripping in a microfluidic T-shaped junction. J. Fluid Mech. 595, 141-161 (2008).

173. Guillot, P. \& Colin, A. Stability of parallel flows in a microchannel after a T junction. Phys. Rev. E - Stat. Nonlinear, Soft Matter Phys. 72, 1-4 (2005).

174. Liontas, R., Ma, K., Hirasaki, G. J. \& Biswal, S. L. Neighbor-induced bubble pinch-off: Novel mechanisms of in situ foam generation in microfluidic channels. Soft Matter 9, 10971-10984 (2013).

175. Link, D. R., Anna, S. L., Weitz, D. A. \& Stone, H. A. Geometrically mediated breakup of drops in 
microfluidic devices. Phys. Rev. Lett. 92, 4 (2004).

176. Li, W. et al. Microfluidic study of fast gas-liquid reactions. J. Am. Chem. Soc. 134, 3127-3132 (2012).

177. Versluis, M. Microbubble acoustic surface cleaning. J. Acoust. Soc. Am. 131, 3337-3337 (2012).

178. Agarwal, A., Ng, W. J. \& Liu, Y. Principle and applications of microbubble and nanobubble technology for water treatment. Chemosphere 84, 1175-1180 (2011).

179. De Gennes, P. G. On fluid/wall slippage. Langmuir 18, 3413-3414 (2002).

180. Kim, C., Qin, R., Xu, J. S., Wang, L. V. \& Xu, R. Multifunctional microbubbles and nanobubbles for photoacoustic and ultrasound imaging. J. Biomed. Opt. 15, 010510 (2010).

181. Yin, T. et al. Nanobubbles for enhanced ultrasound imaging of tumors. Int. J. Nanomedicine 7, 895904 (2012).

182. De Leon, A. et al. Contrast enhanced ultrasound imaging by nature-inspired ultrastable echogenic nanobubbles. Nanoscale 11, 15647-15658 (2019).

183. Nirmalkar, N., Pacek, A. W. \& Barigou, M. Bulk nanobubbles from acoustically cavitated aqueous organic solvent mixtures. Langmuir 35, 2188-2195 (2019).

184. Xing, Z. et al. The fabrication of novel nanobubble ultrasound contrast agent for potential tumor imaging. Nanotechnology 21, 145607 (2010).

185. Parker, J. L., Claesson, P. M. \& Attard, P. Bubbles, cavities, and the long-ranged attraction between hydrophobic surfaces. J. Phys. Chem. 98, 8468-8480 (1994).

186. Zhang, X. H. et al. Degassing and temperature effects on the formation of nanobubbles at the mica/water interface. Langmuir 20, 3813-3815 (2004).

187. Chen, Q., Luo, L. \& White, H. S. Electrochemical generation of a hydrogen bubble at a recessed platinum nanopore electrode. Langmuir 31, 4573-4581 (2015).

188. Lukianova-Hleb, E. Y. et al. Intraoperative diagnostics and elimination of residual microtumours with plasmonic nanobubbles. Nat. Nanotechnol. 11, 525-532 (2016).

189. Castro-Hernández, E., van Hoeve, W., Lohse, D. \& Gordillo, J. M. Microbubble generation in a coflow device operated in a new regime. Lab Chip 11, 2023-2029 (2011).

190. Xiang, N., Yi, H., Chen, K., Wang, S. \& Ni, Z. Investigation of the maskless lithography technique for the rapid and cost-effective prototyping of microfluidic devices in laboratories. $J$. Micromechanics Microengineering 23, (2013).

191. Mo, S., Coussios, C. C., Seymour, L. \& Carlisle, R. Ultrasound-enhanced drug delivery for cancer. Expert Opinion on Drug Delivery 9, 1525-1538 (2012).

192. Park, J. Il et al. Microbubbles loaded with nanoparticles: A route to multiple imaging modalities. ACS Nano 4, 6579-6586 (2010). 
193. Upadhyay, A. \& Dalvi, S. V. Microbubble formulations: Synthesis, stability, modeling and biomedical applications. Ultrasound in Medicine and Biology 45, 301-343 (2019).

194. Cai, W. Bin et al. The optimized fabrication of nanobubbles as ultrasound contrast agents for tumor imaging. Sci. Rep. 5, 13725 (2015).

195. Hernandez, C. et al. Sink or float? Characterization of shell-stabilized bulk nanobubbles using a resonant mass measurement technique. Nanoscale 11, 851-855 (2019).

196. Burg, T. P. et al. Weighing of biomolecules, single cells and single nanoparticles in fluid. Nature 446, 1066-1069 (2007).

197. Godin, M. et al. Using buoyant mass to measure the growth of single cells. Nat. Methods 7, 387390 (2010).

198. Haghi, H., Sojahrood, A. J. \& Kolios, M. C. Collective nonlinear behavior of interacting polydisperse microbubble clusters. Ultrason. Sonochem. 58, 104708 (2019).

199. Gormley, C. A. et al. Fibrin-targeted polymerized shell microbubbles as potential theranostic agents for surgical adhesions. Langmuir 35, 10061-10067 (2019).

200. Théry, C. et al. Proteomic analysis of dendritic cell-derived exosomes: A secreted subcellular compartment distinct from apoptotic vesicles. J. Immunol. 166, 7309-7318 (2001).

201. Salari, A. et al. Dancing with the cells: Acoustic microflows generated by oscillating cells. Small 16, 1903788 (2019).

202. Zervantonakis, I. K. \& Arvanitis, C. D. Controlled drug release and chemotherapy response in a novel acoustofluidic 3D tumor platform. Small 12, 2616-2626 (2016).

203. Zhang, J. et al. Surface acoustic waves enable rotational manipulation of Caenorhabditis elegans. Lab Chip 19, 984-992 (2019).

204. Wu, M. et al. Separating extracellular vesicles and lipoproteins via acoustofluidics. Lab Chip 19, 1174-1182 (2019).

205. Zhao, S. et al. On-chip stool liquefaction via acoustofluidics. Lab Chip 19, 941-947 (2019).

206. Orbay, S. et al. Mixing high-viscosity fluids via acoustically driven bubbles. J. Micromechanics Microengineering 27, 015008 (2017).

207. Huang, P.-H. et al. An acoustofluidic micromixer based on oscillating sidewall sharp-edges. Lab Chip 13, 3847-3852 (2013).

208. Ahmed, D. et al. Acoustofluidic chemical waveform generator and switch. Anal. Chem. 86, 1180311810 (2014).

209. Huang, P.-H. et al. A reliable and programmable acoustofluidic pump powered by oscillating sharpedge structures. Lab Chip 14, 4319-4323 (2014).

210. Tovar, A. R., Patel, M. V. \& Lee, A. P. Lateral air cavities for microfluidic pumping with the use of 
acoustic energy. Microfluid. Nanofluidics 10, 1269-1278 (2011).

211. Barnkob, R. et al. Acoustically driven fluid and particle motion in confined and leaky systems. Phys. Rev. Appl. 9, 014027 (2018).

212. Huang, P. H. et al. A sharp-edge-based acoustofluidic chemical signal generator. Lab Chip 18, 1411-1421 (2018).

213. Liu, X. \& Wu, J. Acoustic microstreaming around an isolated encapsulated microbubble. J. Acoust. Soc. Am. 125, 1319-1330 (2009).

214. Doinikov, A. a \& Bouakaz, A. Acoustic microstreaming around a gas bubble. J. Acoust. Soc. Am. 127, 703-709 (2010).

215. Lighthill, J. Acoustic streaming in the ear itself. J. Fluid Mech. 239, 551 (1992).

216. Kotas, C. W., Rogers, P. H. \& Yoda, M. Acoustically induced streaming flows near a model cod otolith and their potential implications for fish hearing. J. Acoust. Soc. Am. 130, 1049-1059 (2011).

217. Hassan, E.-S. A suggested role for secondary flow in the stimulation of the cochlear hair cell. Biol. Cybern. 53, 109-119 (1985).

218. Ehlers, K. M. \& Koiller, J. Could cell membranes produce acoustic streaming? Making the case for Synechococcus self-propulsion. Math. Comput. Model. 53, 1489-1504 (2011).

219. Koiller, J., Ehlers, K. M. \& Chalub, F. Acoustic streaming, the "small invention" of cyanobacteria? Arbor 186, 1089-1115 (2010).

220. Ehlers, K. M. \& Koiller, J. Micro-swimming without flagella: Propulsion by internal structures. Regul. Chaotic Dyn. 16, 623-652 (2011).

221. Kaynak, M. et al. Acoustic actuation of bioinspired microswimmers. Lab Chip 17, 395-400 (2017).

222. Ahmed, D. et al. Selectively manipulable acoustic-powered microswimmers. Sci. Rep. 5, 9744 (2015).

223. Leibacher, I., Hahn, P. \& Dual, J. Acoustophoretic cell and particle trapping on microfluidic sharp edges. Microfluid. Nanofluidics 19, 923-933 (2015).

224. Tinevez, J. Y. et al. TrackMate: An open and extensible platform for single-particle tracking. Methods 115, 80-90 (2017).

225. Su, Z., Ye, L. \& Lu, Y. Guided Lamb waves for identification of damage in composite structures: A review. J. Sound Vib. 295, 753-780 (2006).

226. Tsuji, K. et al. Effects of different cell-detaching methods on the viability and cell surface antigen expression of synovial mesenchymal stem cells. Cell Transplant. 26, 1089-1102 (2017).

227. Malluci, L., Wells, V. \& Young, M. R. Effect of trypsin on cell volume and mass. Nat. New Biol. 239, 53-55 (1972).

228. Raat, N. J., De Smet, P., van Driessche, W., Bindels, R. J. \& Van Os, C. H. Measuring volume 
perturbation of proximal tubular cells in primary culture with three different techniques. $A m . J$. Physiol. Physiol. 271, C235-C241 (1996).

229. Guo, M. et al. Cell volume change through water efflux impacts cell stiffness and stem cell fate. Proc. Natl. Acad. Sci. U. S. A. 114, E8618-E8627 (2017).

230. Lambert, I. H., Hoffmann, E. K. \& Pedersen, S. F. Cell volume regulation: Physiology and pathophysiology. Acta Physiol. 194, 255-282 (2008).

231. Ayee, M. A. A., LeMaster, E., Teng, T., Lee, J. \& Levitan, I. Hypotonic challenge of endothelial cells increases membrane stiffness with no effect on tether force. Biophys. J. 114, 929-938 (2018).

232. Kim, S.-O., Kim, J., Okajima, T. \& Cho, N.-J. Mechanical properties of paraformaldehyde-treated individual cells investigated by atomic force microscopy and scanning ion conductance microscopy. Nano Converg. 4, 5 (2017).

233. Moeendarbary, E. \& Harris, A. R. Cell mechanics: Principles, practices, and prospects. Wiley Interdiscip. Rev. Syst. Biol. Med. 6, 371-388 (2014).

234. Yamane, Y. et al. Quantitative analyses of topography and elasticity of living and fixed astrocytes. J. Electron Microsc. (Tokyo). 49, 463-471 (2000).

235. Steltenkamp, S., Rommel, C., Wegener, J. \& Janshoff, A. Membrane stiffness of animal cells challenged by osmotic stress. Small 2, 1016-1020 (2006).

236. Grady, M. E., Composto, R. J. \& Eckmann, D. M. Cell elasticity with altered cytoskeletal architectures across multiple cell types. J. Mech. Behav. Biomed. Mater. 61, 197-207 (2016).

237. Guillou, L. et al. Measuring cell viscoelastic properties using a microfluidic extensional flow device. Biophys. J. 111, 2039-2050 (2016).

238. Girardo, S. et al. Standardized microgel beads as elastic cell mechanical probes. J. Mater. Chem. B 6, 6245-6261 (2018).

239. Wu, P. H. et al. A comparison of methods to assess cell mechanical properties. Nat. Methods 15, 491-498 (2018).

240. Lekka, M. Discrimination between normal and cancerous cells using AFM. Bionanoscience 6, 6580 (2016).

241. Achterberg, V. F. et al. The nano-scale mechanical properties of the extracellular matrix regulate dermal fibroblast function. J. Invest. Dermatol. 134, 1862-1872 (2014).

242. Lipinski, C. A., Lombardo, F., Dominy, B. W. \& Feeney, P. J. Experimental and computational approaches to estimate solubility and permeability in drug discovery and development settings. $A d v$. Drug Deliv. Rev. 23, 3-25 (1997).

243. Bareford, L. M. \& Swaan, P. W. Endocytic mechanisms for targeted drug delivery. Adv. Drug Deliv. Rev. 59, 748-758 (2007). 
244. Cardarelli, F. et al. The intracellular trafficking mechanism of Lipofectamine-based transfection reagents and its implication for gene delivery. Sci. Rep. 6, 25879 (2016).

245. Kumar, P., Nagarajan, A. \& Uchil, P. D. Lipofection. Cold Spring Harb. Protoc. 2019, (2019).

246. Bolhassani, A., Khavari, A. \& Oraf, Z. Electroporation - advantages and drawbacks for delivery of drug, gene and vaccine. in Application of Nanotechnology in Drug Delivery (InTech, 2014). doi: $10.5772 / 58376$

247. Lentacker, I., De Cock, I., Deckers, R., De Smedt, S. C. \& Moonen, C. T. W. Understanding ultrasound induced sonoporation: Definitions and underlying mechanisms. Adv. Drug Deliv. Rev. 72, 49-64 (2014).

248. Freese, C. et al. In vitro investigation of silica nanoparticle uptake into human endothelial cells under physiological cyclic stretch. Part. Fibre Toxicol. 11, 1-12 (2014).

249. Liu, Y., Yan, J. \& Prausnitz, M. R. Can ultrasound enable efficient intracellular uptake of molecules? A retrospective literature review and analysis. Ultrasound Med. Biol. 38, 876-888 (2012).

250. Wang, J. T. H., Teasdale, R. D. \& Liebl, D. Macropinosome quantitation assay. Methods X 1, 36-41 (2014).

251. Mcneil, P. L. Incorporation of macromolecules into living cells. Methods Cell Biol. 29, 153-173 (1988).

252. Doherty, G. J. \& McMahon, H. T. Mechanisms of endocytosis. Annu. Rev. Biochem. 78, 857-902 (2009).

253. Pavelka, M., Roth, J., Pavelka, M. \& Roth, J. Fluid-phase endocytosis and phagocytosis. in Functional Ultrastructure 104-105 (Springer Vienna, 2010). doi:10.1007/978-3-211-99390-3_54

254. Li, L. et al. The effect of the size of fluorescent dextran on its endocytic pathway. Cell Biol. Int. 39, 531-9 (2015).

255. Hallermann, S. Calcium channels for endocytosis. J. Physiol. 592, 3343-3344 (2014).

256. Wu, L.-G., Hamid, E., Shin, W. \& Chiang, H.-C. Exocytosis and endocytosis: Modes, functions, and coupling mechanisms. Annu. Rev. Physiol. 76, 301-331 (2013).

257. Wu, X.-S. et al. $\mathrm{Ca}(2+)$ and calmodulin initiate all forms of endocytosis during depolarization at a nerve terminal. Nat. Neurosci. 12, 1003-1010 (2009).

258. Leopold, P. L. Endosomal escape pathways for delivery of biologics. Lysosomes Biol. Dis. Ther. 151, 383-407 (2016).

259. Copper, Geoffrey and Hausman, R. Cell: A molecular approach. (Sinauer Associates, 2007).

260. Fekri, F. et al. Targeted enhancement of flotillin-dependent endocytosis augments cellular uptake and impact of cytotoxic drugs. Sci. Rep. 9, (2019).

261. Ando, J. \& Kamiya, A. Blood flow and vascular endothelial cell function. Front. Med. Biol. Eng. 5, 
245-64 (1993).

262. Geiger, R. V., Berk, B. C., Alexander, R. W. \& Nerem, R. M. Flow-induced calcium transients in single endothelial cells: Spatial and temporal analysis. Am. J. Physiol. Physiol. 262, C1411-C1417 (1992).

263. Tatiparti, K., Sau, S., Kashaw, S. K. \& Iyer, A. K. siRNA delivery strategies: A comprehensive review of recent developments. Nanomaterials 7, 77 (2017).

264. Alberts, B. et al. Molecular biology of the cell. (W. W. Norton \& Company, 2015).

265. Schumann, K. et al. Generation of knock-in primary human T cells using Cas9 ribonucleoproteins. Proc. Natl. Acad. Sci. U. S. A. 112, 10437-10442 (2015).

266. Tsoi, M. et al. Characterization of condensed plasmid DNA models for studying the direct effect of ionizing radiation. Biophys. Chem. 147, 104-10 (2010).

267. Cohen, S. N. \& Miller, C. A. Multiple molecular species of circular R-factor DNA isolated from Escherichia coli. Nature 224, 1273-7 (1969).

268. Tang, M. X. \& Szoka, F. C. The influence of polymer structure on the interactions of cationic polymers with DNA and morphology of the resulting complexes. Gene Ther. 4, 823-32 (1997).

269. Huotari, J. \& Helenius, A. Endosome maturation. EMBO J. 30, 3481-3500 (2011).

270. Taylor, M. J., Perrais, D. \& Merrifield, C. J. A High Precision Survey of the Molecular Dynamics of Mammalian Clathrin-Mediated Endocytosis. PLoS Biol. 9, e1000604 (2011).

271. Sahay, G., Alakhova, D. Y. \& Kabanov, A. V. Endocytosis of nanomedicines. J. Control. Release 145, 182-195 (2010).

272. Mintzer, M. A. \& Simanek, E. E. Nonviral vectors for gene delivery. Chemical Reviews 109, 259302 (2009).

273. Heyden, S. \& Ortiz, M. Investigation of the influence of viscoelasticity on oncotripsy. Comput. Methods Appl. Mech. Eng. 314, 314-322 (2017).

274. Mittelstein, D. R. et al. Selective ablation of cancer cells with low intensity pulsed ultrasound. Appl. Phys. Lett. 116, (2020).

275. Yoon, S. et al. Direct and sustained intracellular delivery of exogenous molecules using acoustictransfection with high frequency ultrasound. Sci. Rep. 6, 1-11 (2016).

276. Karshafian, R., Bevan, P. D., Williams, R., Samac, S. \& Burns, P. N. Sonoporation by ultrasoundactivated microbubble contrast agents: Effect of acoustic exposure parameters on cell membrane permeability and cell viability. Ultrasound Med. Biol. 35, 847-860 (2009).

277. Cross, S. E. et al. AFM-based analysis of human metastatic cancer cells. Nanotechnology 19, (2008).

278. Xu, W. et al. Cell stiffness is a biomarker of the metastatic potential of ovarian cancer cells. PLoS One 7, (2012). 
279. Ekemen, Z. et al. Fabrication of biomaterials via controlled protein bubble generation and manipulation. Biomacromolecules 12, 4291-4300 (2011).

280. Feng, H. et al. Visualization of two-phase reacting flow behavior in a gas-liquid-solid microreactor. React. Chem. Eng. 4, 715-723 (2019).

281. Zhang, J., Teixeira, A. R., Zhang, H. \& Jensen, K. F. Determination of fast gas-liquid reaction kinetics in flow. React. Chem. Eng. 5, 51-57 (2020).

282. Hua, T. \& Hartman, R. L. Computational fluid dynamics of DNA origami folding in microfluidics. React. Chem. Eng. 4, 818-827 (2019).

283. Sarna, M. et al. Cell elasticity is an important indicator of the metastatic phenotype of melanoma cells. Exp. Dermatol. 23, 813-818 (2014).

284. Nama, N., Huang, P.-H., Huang, T. J. \& Costanzo, F. Investigation of acoustic streaming patterns around oscillating sharp edges. Lab Chip 14, 2824-2836 (2014).

285. Muller, P. B., Barnkob, R., Jensen, M. J. H. \& Bruus, H. A numerical study of microparticle acoustophoresis driven by acoustic radiation forces and streaming-induced drag forces. Lab Chip 12, 4617 (2012).

286. Meijering, B. D. M. et al. Ultrasound and microbubble-targeted delivery of macromolecules is regulated by induction of endocytosis and pore formation. Circ. Res. 104, 679-687 (2009). 